\title{
iTOUGH2 \\ Command Reference
}

\author{
Stefan Finsterle \\ Earth Sciences Division \\ Lawrence Berkeley National Laboratory \\ University of California \\ Berkeley, CA 94720
}

June 2002

This work was supported, in part, by the U.S. Dept. of Energy under Contract No. DE-AC03-76SF00098, and by a grant from the Swiss National Cooperative for the Disposal of Radioactive Waste (Nagra), Wettingen, Switzerland. 


\section{DISCLAIMER}

This document was prepared as an account of work sponsored by the United States Government. While this document is believed to contain correct information, neither the United States Government nor any agency thereof, nor The Regents of the University of California, nor any of their employees, makes any warranty, express or implied, or assumes any legal responsibility for the accuracy, completeness, or usefulness of any information, apparatus, product, or process disclosed, or represents that its use would not infringe privately owned rights. Reference herein to any specific commercial product, process, or service by its trade name, trademark, manufacturer, or otherwise, does not necessarily constitute or imply its endorsement, recommendation, or favoring by the United States Government or any agency thereof, or The Regents of the University of California. The views and opinions of authors expressed herein do not necessarily state or reflect those of the United States Government or any agency thereof, or The Regents of the University of California.

Ernest Orlando Lawrence Berkeley National Laboratory is an equal opportunity employer. 


\section{TABLE OF CONTENTS}

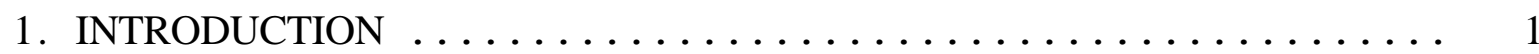

2. iTOUGH2 SUMMARY DESCRIPTION ................. 2

3. BASIC CONCEPTS ....................... 8

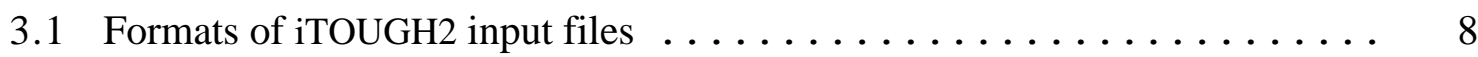

3.2 Basic concepts of iTOUGH2 input language $\ldots \ldots \ldots \ldots \ldots \ldots$

3.3 Main structure of iTOUGH 2 input $\quad \ldots \ldots \ldots \ldots \ldots \ldots \ldots \ldots \ldots$

4. iTOUGH2 COMMANDS $\ldots \ldots \ldots \ldots \ldots \ldots \ldots \ldots \ldots \ldots \ldots \ldots \ldots \ldots$

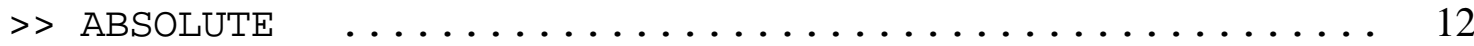

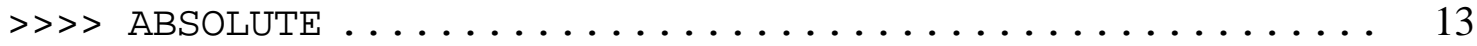

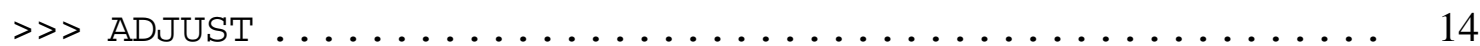

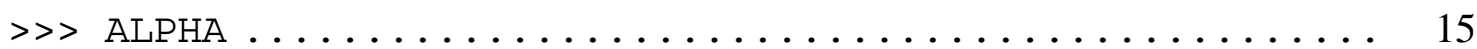

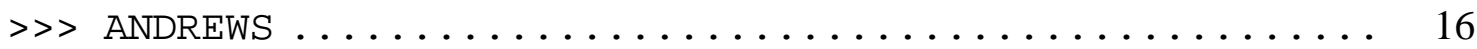

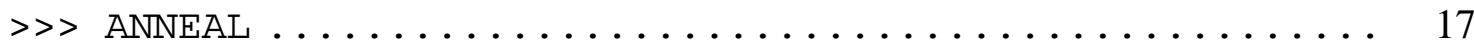

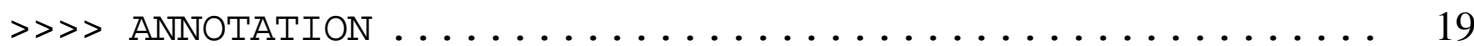

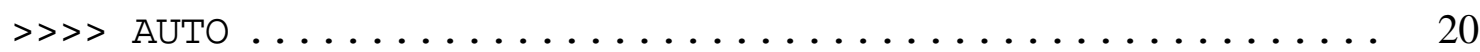

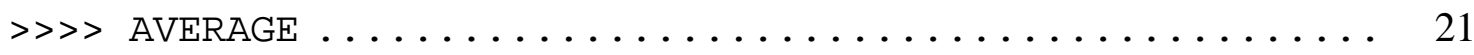

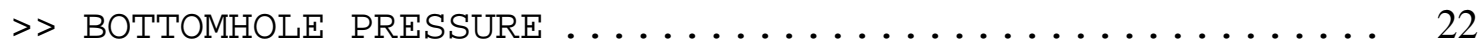

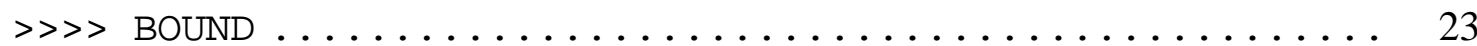

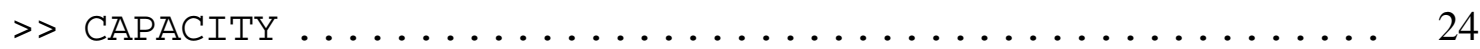

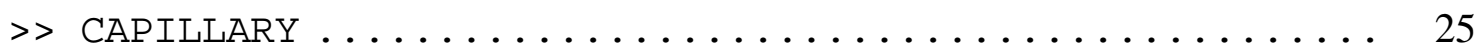

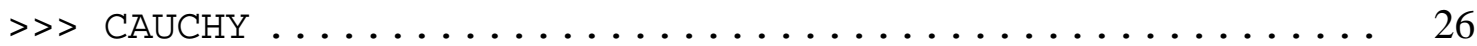

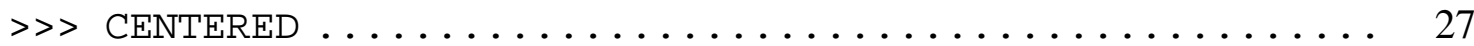

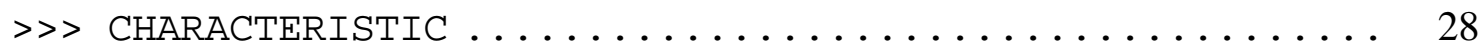

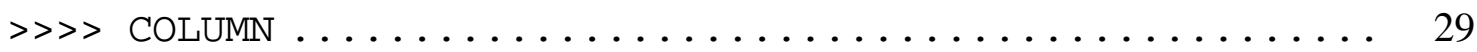

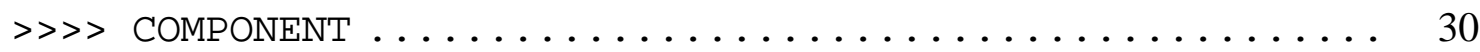

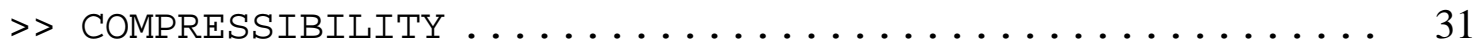

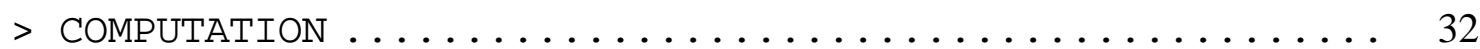

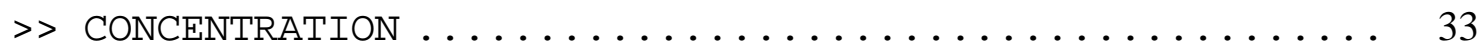

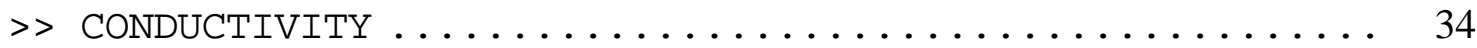

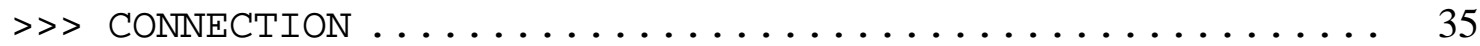

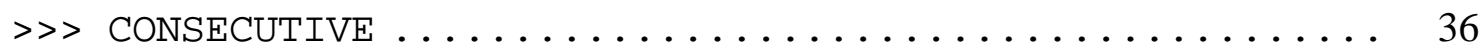

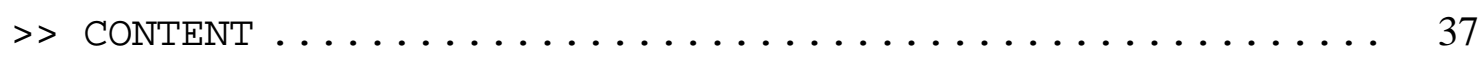

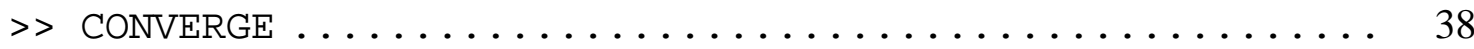

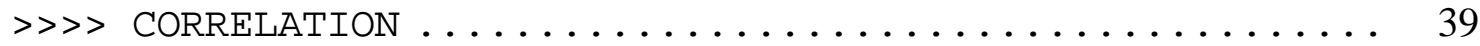

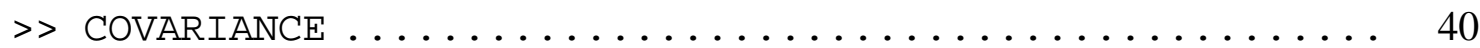

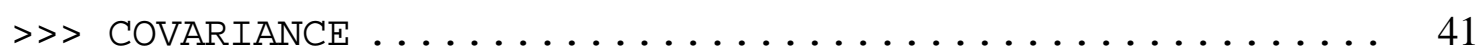

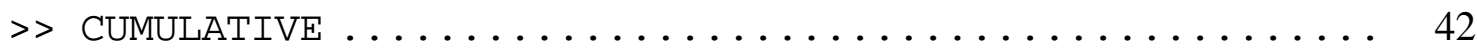

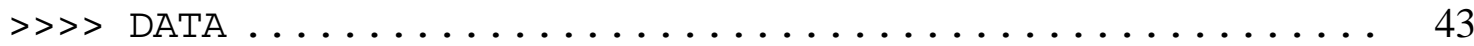

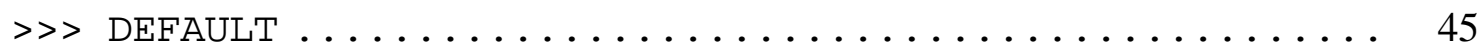

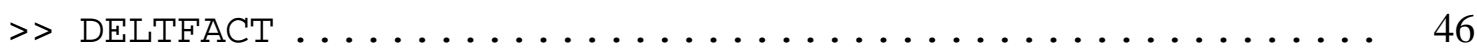




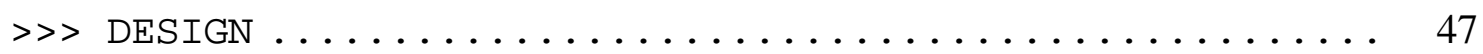

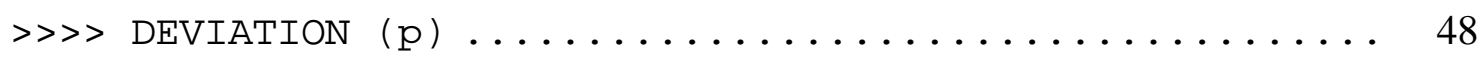

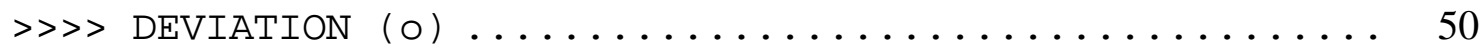

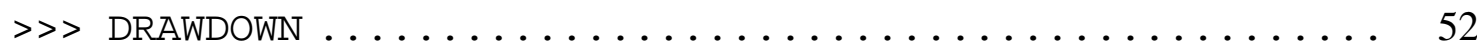

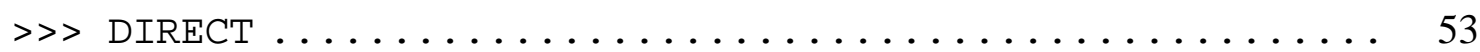

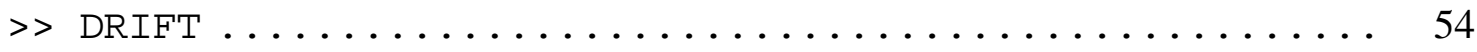

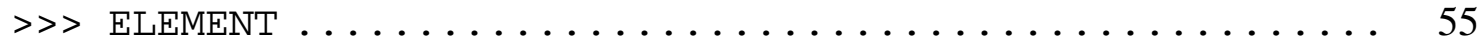

$>>$ EMPIRICAL ORTHOGONAL FUNCTIONS .............. 56

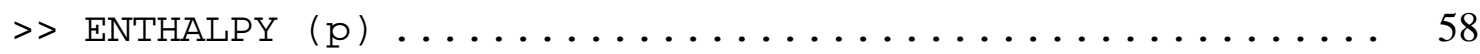

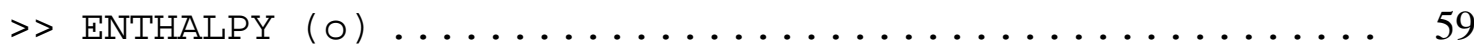

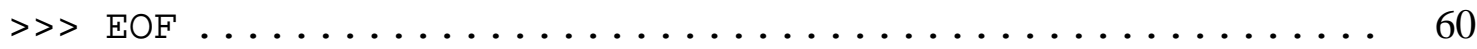

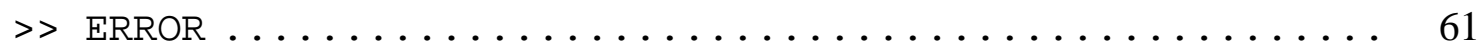

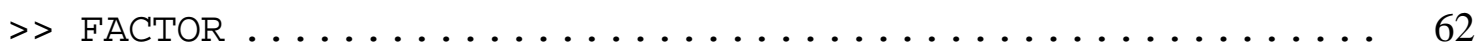

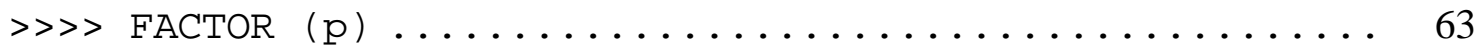

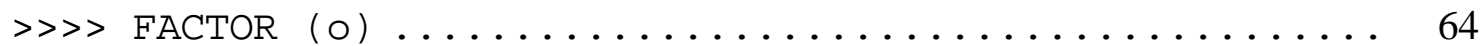

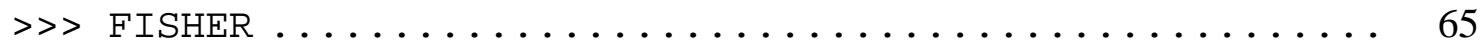

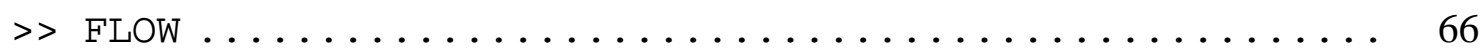

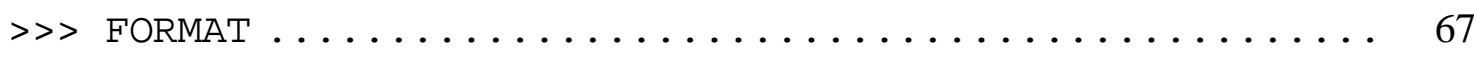

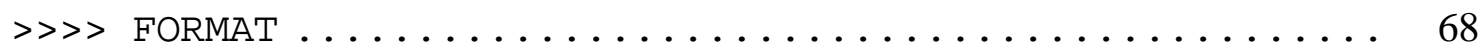

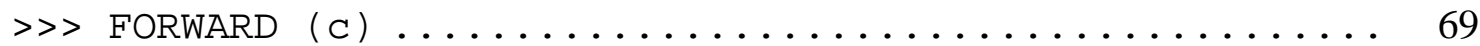

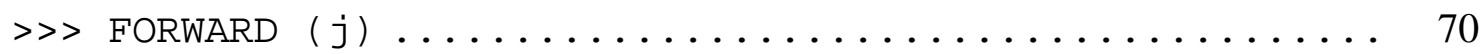

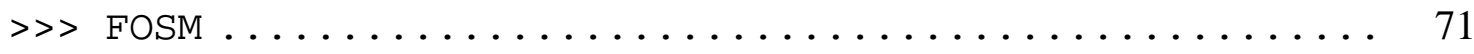

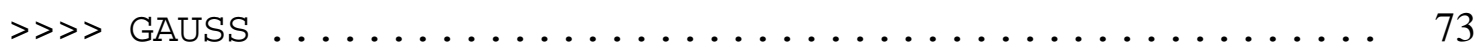

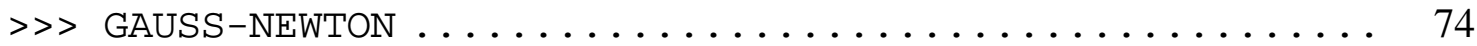

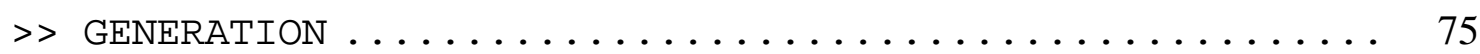

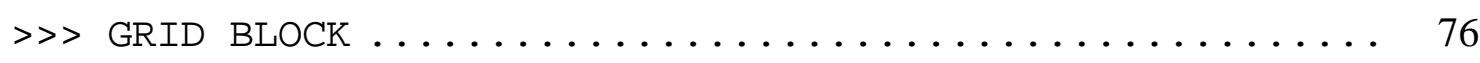

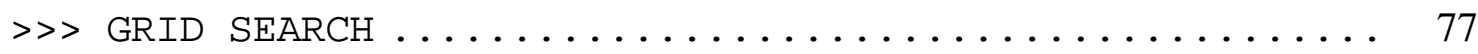

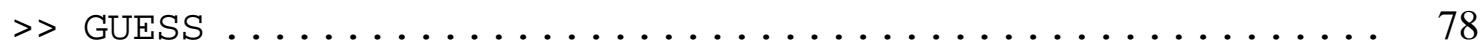

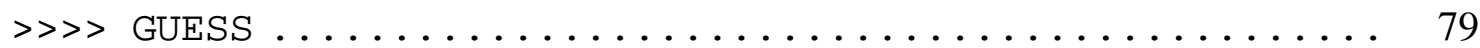

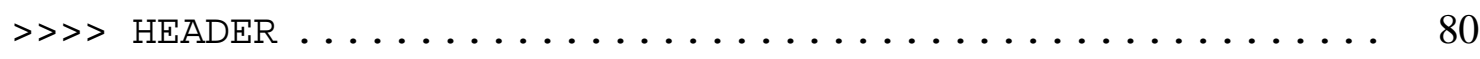

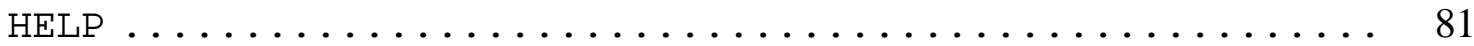

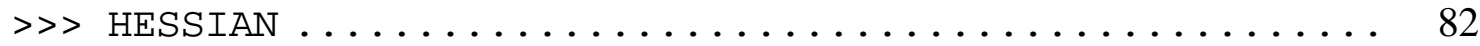

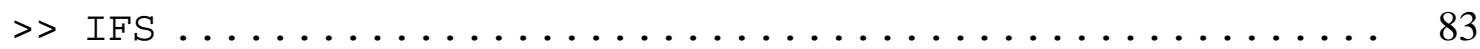

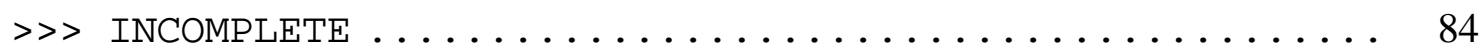

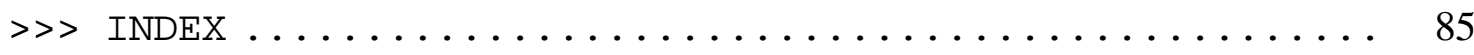

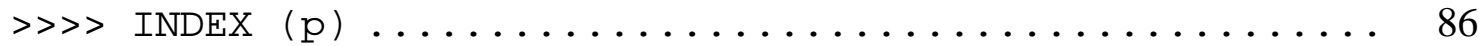

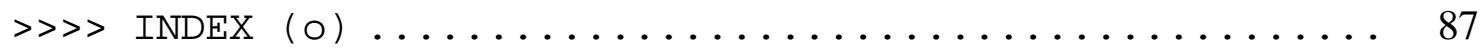

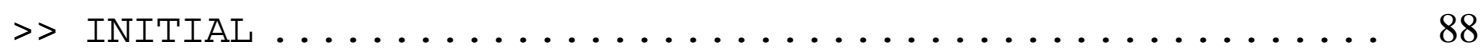

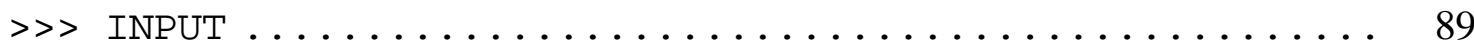

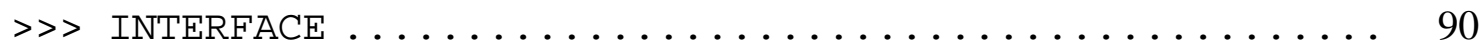

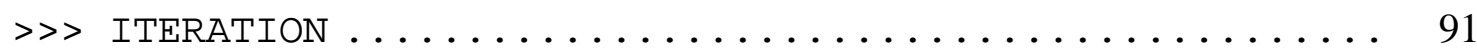

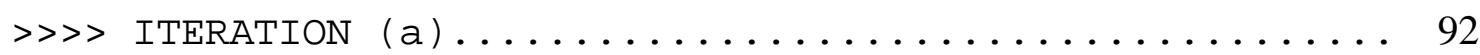

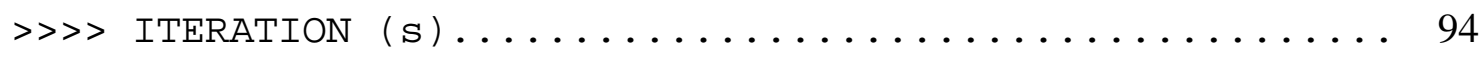

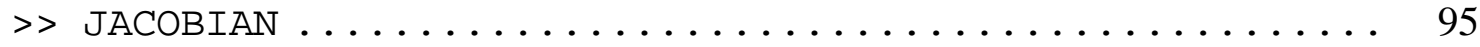

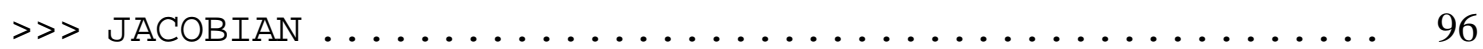




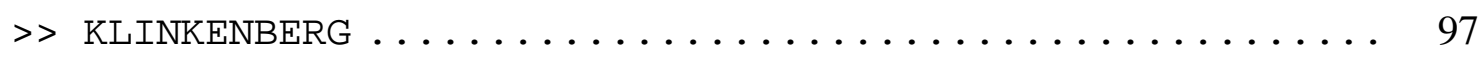

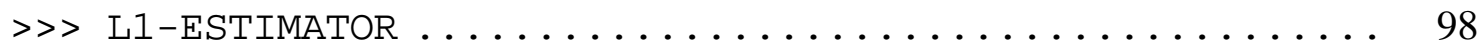

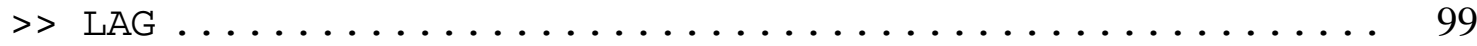

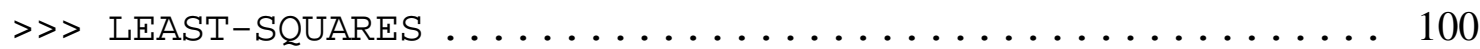

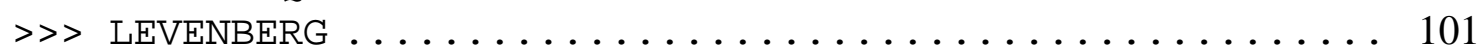

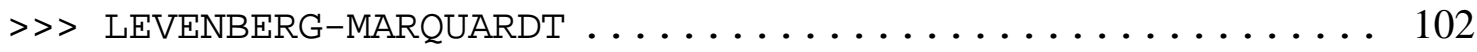

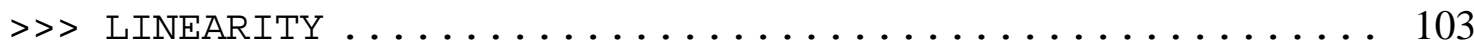

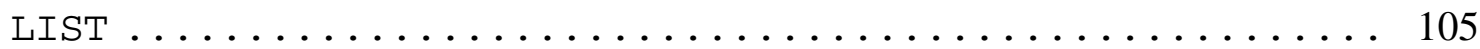

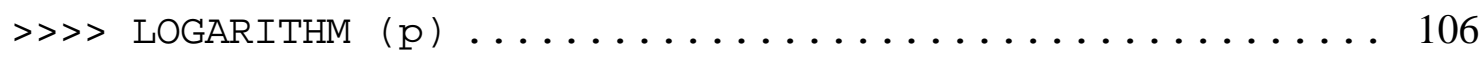

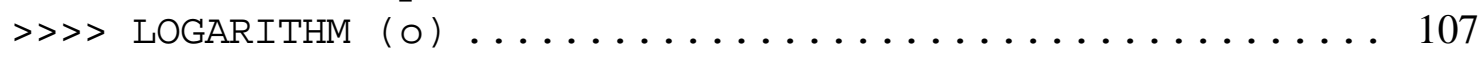

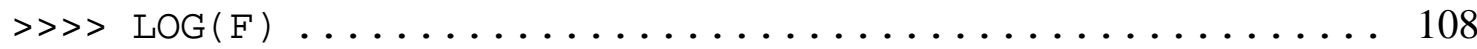

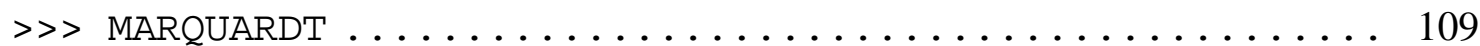

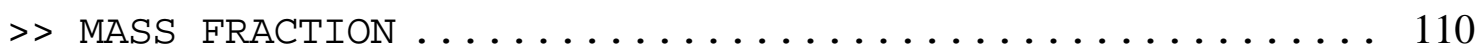

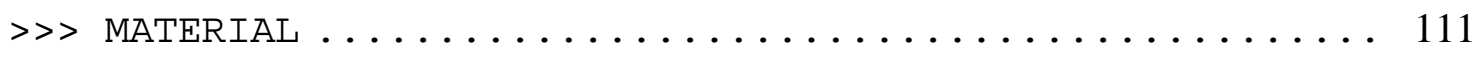

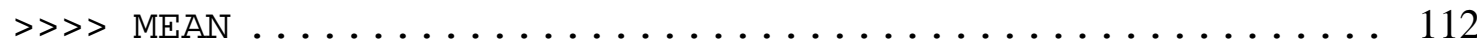

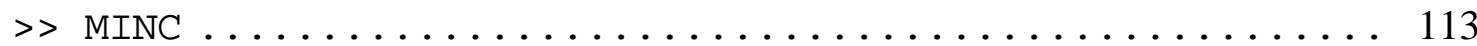

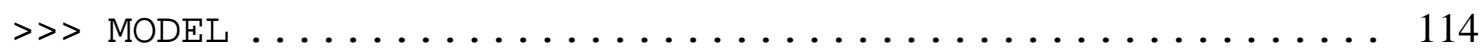

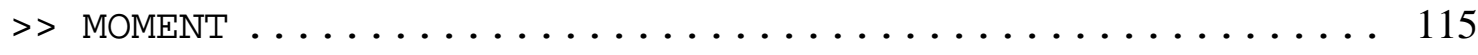

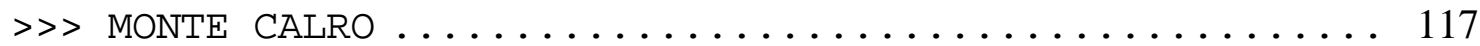

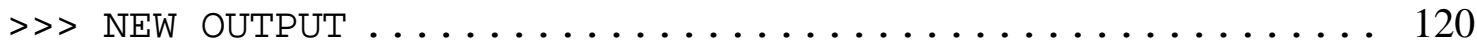

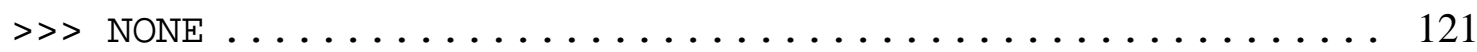

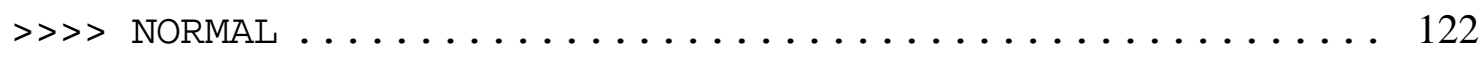

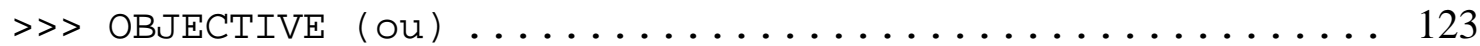

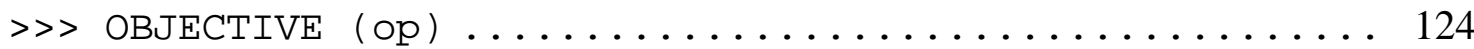

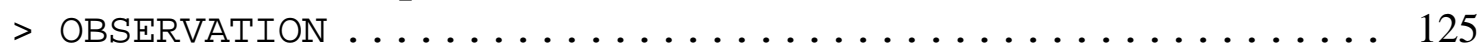

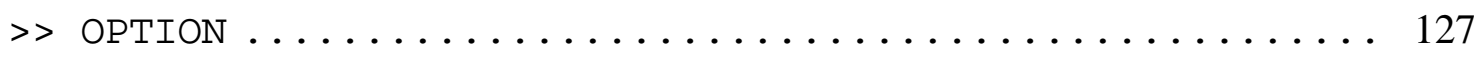

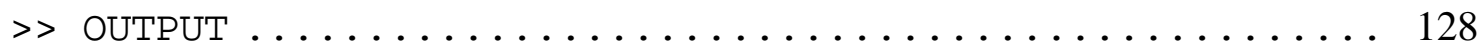

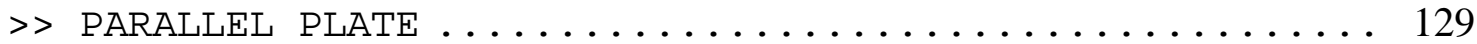

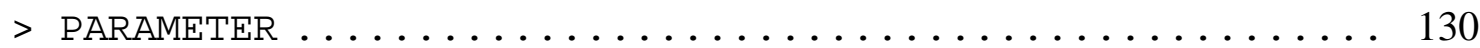

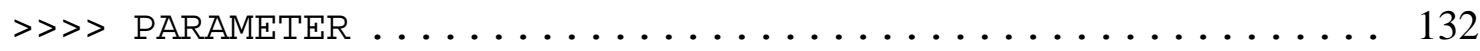

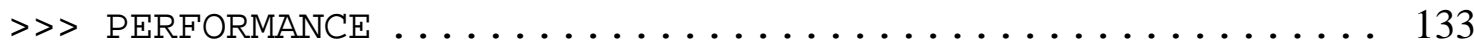

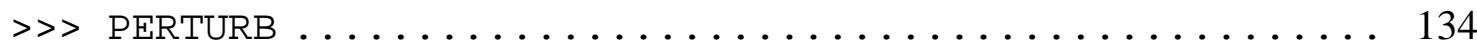

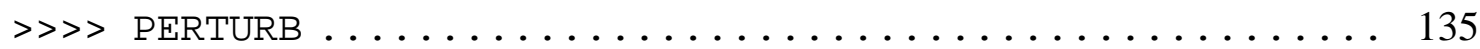

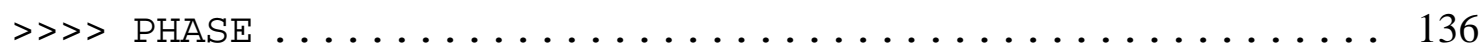

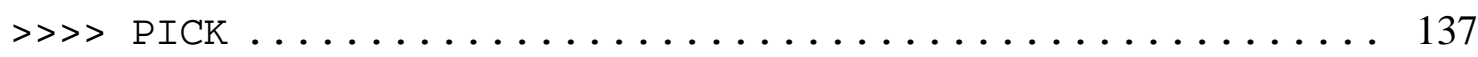

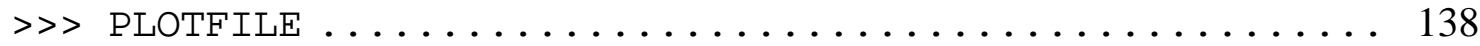

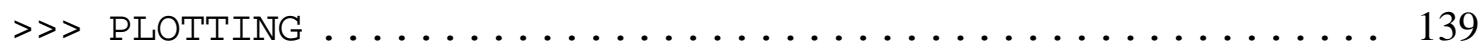

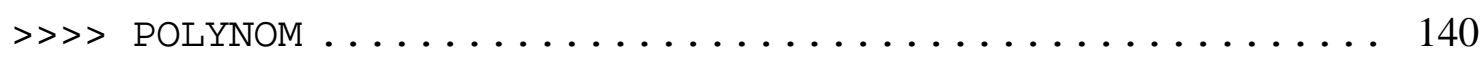

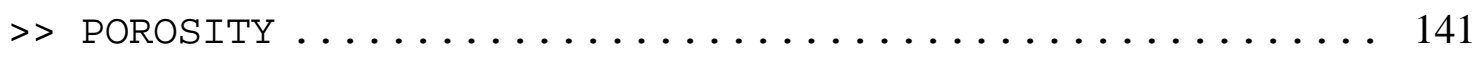

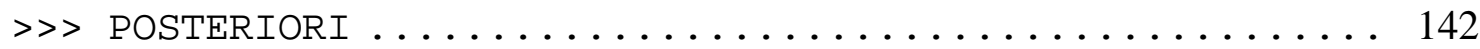

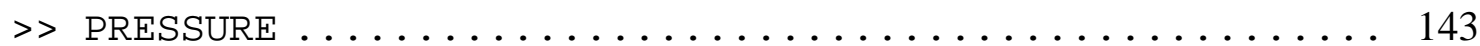

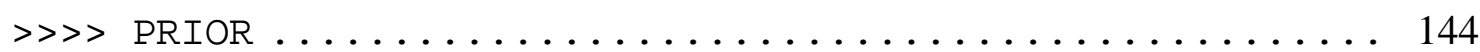

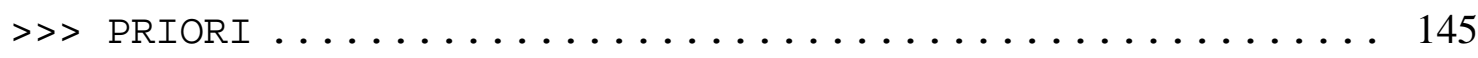

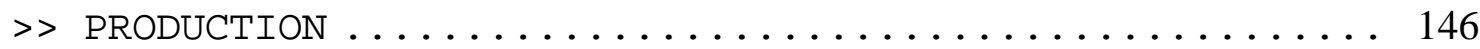

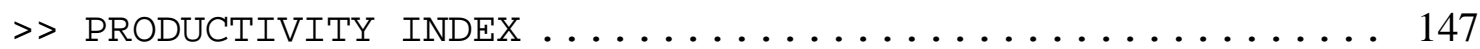




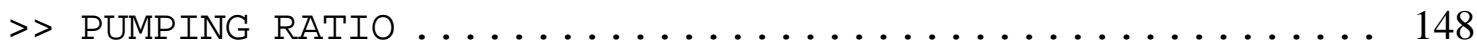

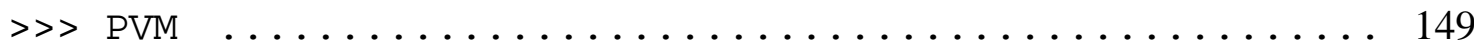

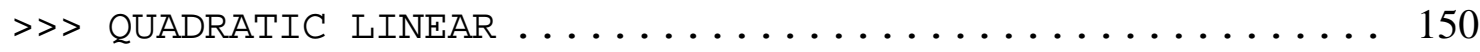

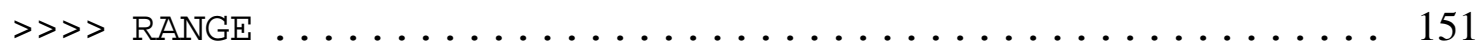

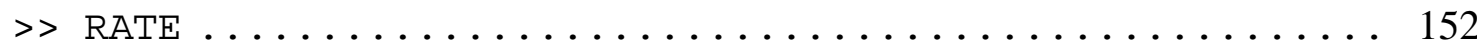

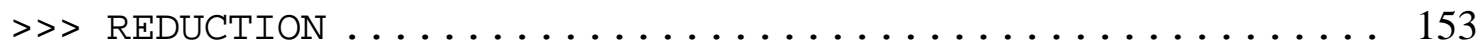

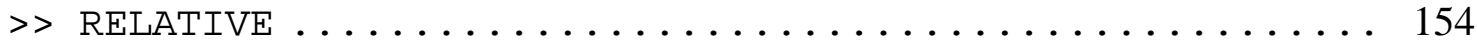

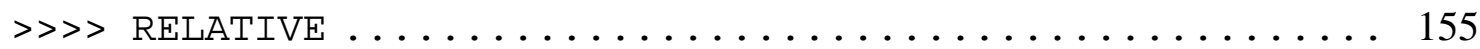

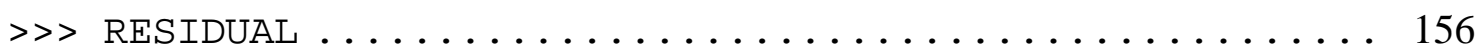

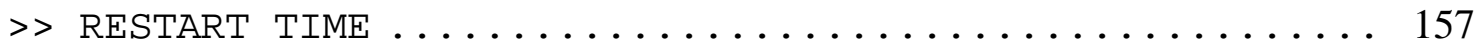

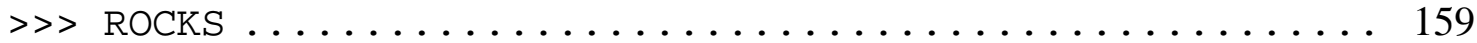

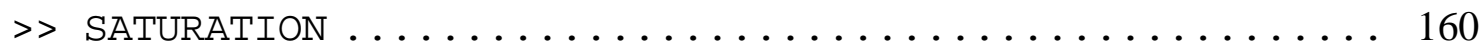

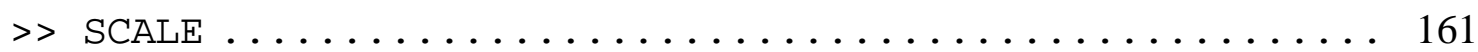

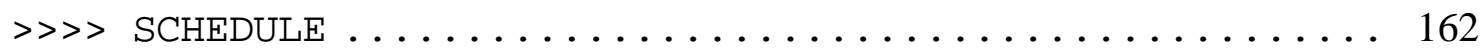

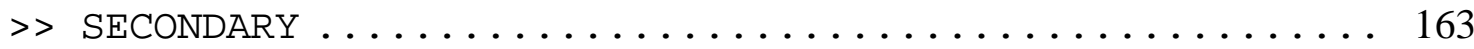

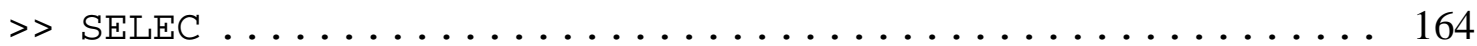

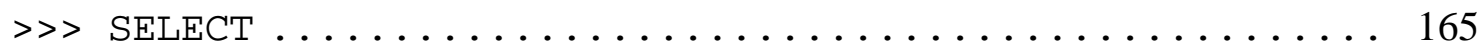

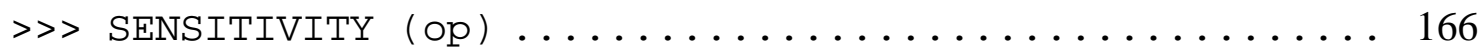

$>>$ SENSITIVITY $(\mathrm{ou}) \ldots \ldots \ldots \ldots \ldots \ldots \ldots \ldots \ldots \ldots \ldots \ldots$

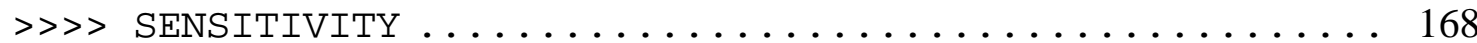

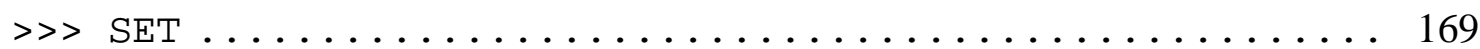

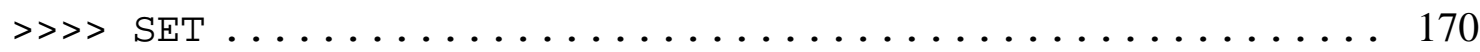

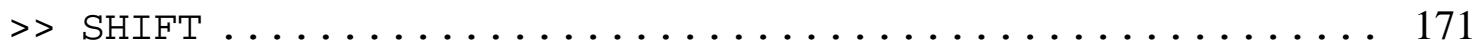

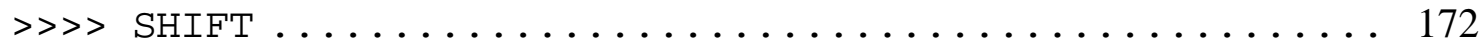

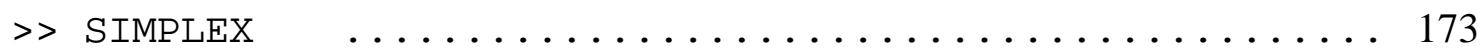

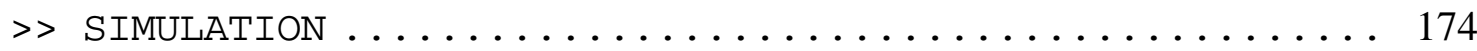

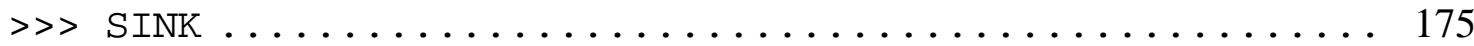

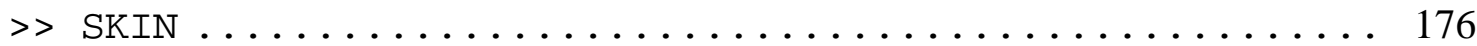

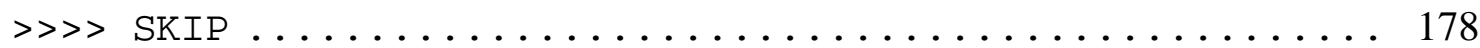

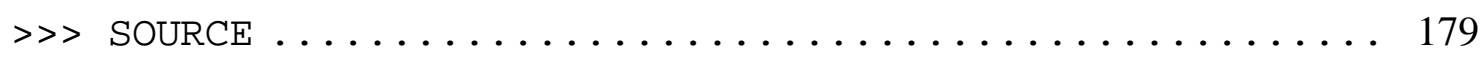

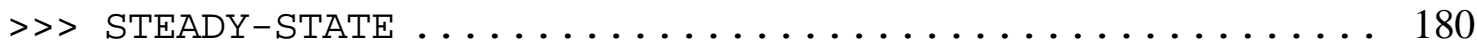

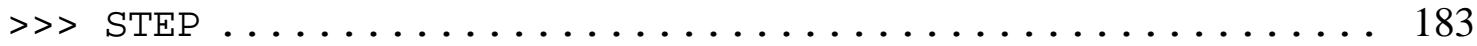

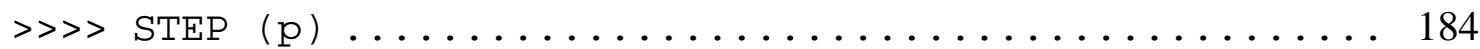

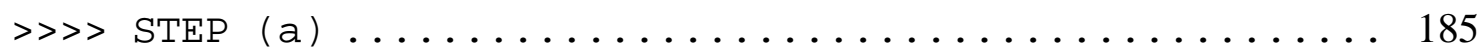

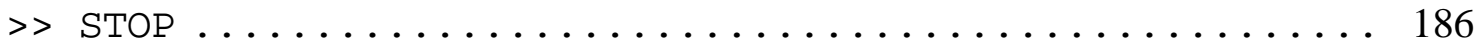

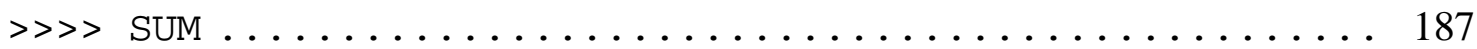

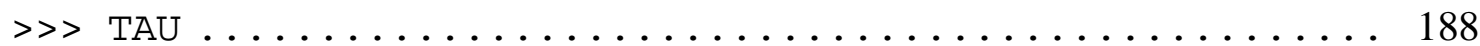

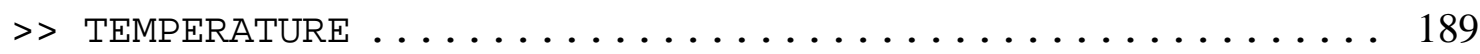

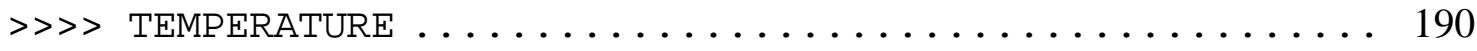

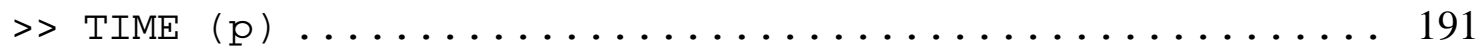

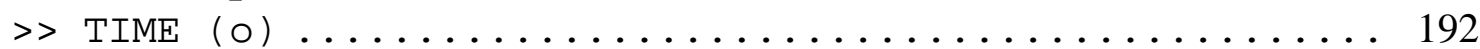

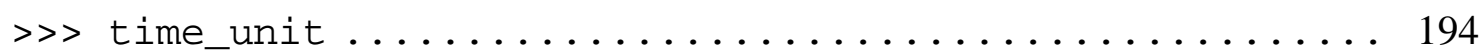

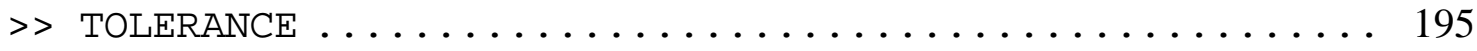

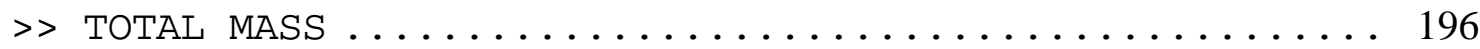

$>>>\mathrm{UNIFORM} \ldots \ldots \ldots \ldots \ldots \ldots \ldots \ldots \ldots \ldots \ldots \ldots \ldots \ldots \ldots$ 


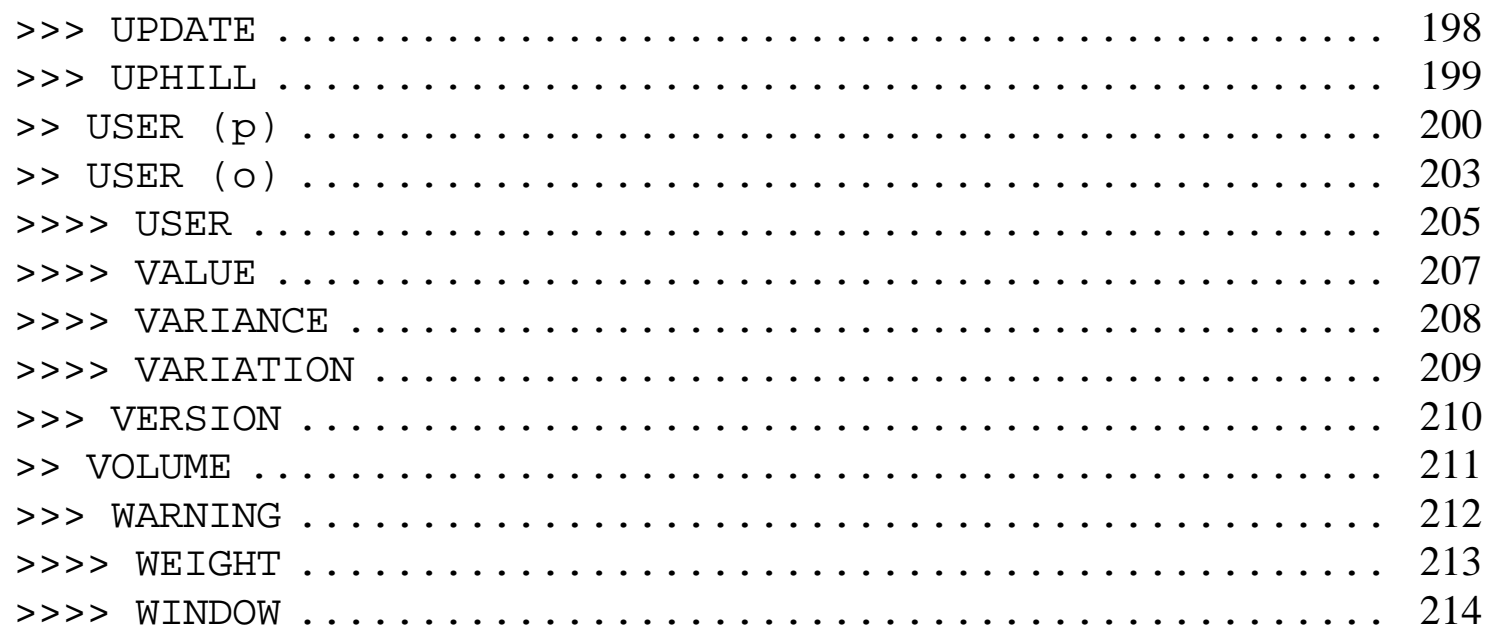

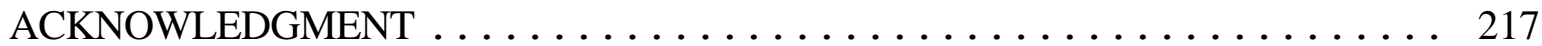

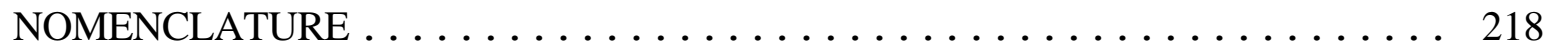

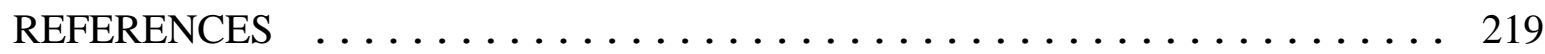

APPENDIX A: TOUGH2 Enhancements ................. 221

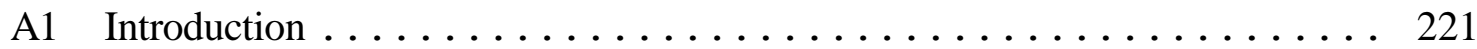

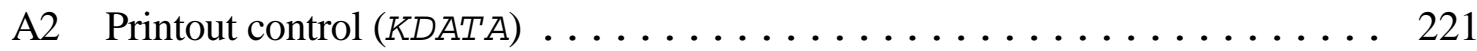

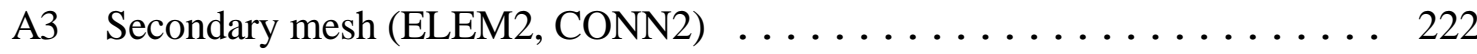

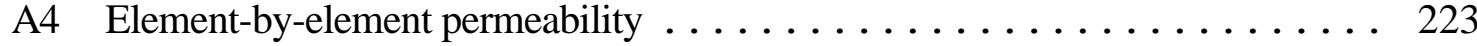

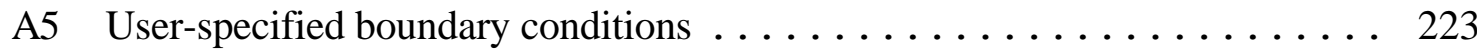

A6 Relative permeability and capillary pressure functions . . . . . . . . . 225

A6.1 Modified Brooks-Corey model .................. 225

A6.2 Modified van Genuchten model . . . . . . . . . . . . . . 227

A7 Reduction fo fracture-matrix interface area . . . . . . . . . . . . . . . . . . 229

A8 Excluding domains from global material balances . . . . . . . . . . . . . . . . . . . . . . . . . . . . . . . . . . . .

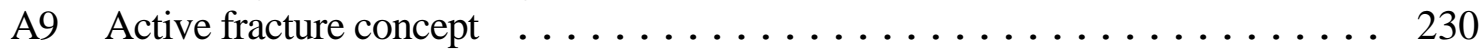

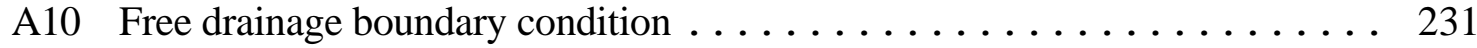

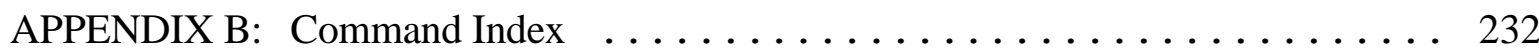




\section{LIST OF FIGURES}

Figure 3.3.1. Main structure of iTOUGH2 input. $\ldots \ldots \ldots \ldots \ldots \ldots$

Figure A3.1. Primary and secondary mesh generation. . . . . . . . . . . 222

Figure A5.2. Subroutine USERBC for specifying time-dependent boundary conditions. . . . . . . . . . . . . . . . . 224

Figure A6.1.1. Modified Brooks-Corey relative permeability and capillary pressure curves. .......................... 226

Figure A6.2.1. Modified van Genuchten relative permeability and capillary pressure

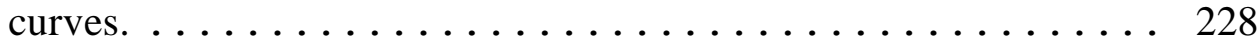




\section{LIST OF TABLES}

Table 2.1. Parameter Transformations .................. 3

Table 2.2. Levenberg-Marquardt Minimization Algorithm $\ldots \ldots \ldots \ldots$

Table A2. Amount of Printout as a Function of Variable $\quad$ KDATA $\ldots \ldots \ldots 221$

Table A6.1.1. Input Parameters for Modified Brooks-Corey Model . . . . . . . . . . 226

Table A6.2.1. Input Parameters for Modified van Genuchten Model . . . . . . . . . . . 228

Table A7.1. Option for Reducing Fracture-Matrix Interface Area . . . . . . . . . 229 


\section{INTRODUCTION}

This report contains a detailed description of all iTOUGH 2 commands. It complements the "iTOUGH2 User's Guide" [Finsterle, 1999a], and the collection of iTOUGH2 sample problems [Finsterle, 1999c].

iTOUGH2 is a program for parameter estimation, sensitivity analysis, and uncertainty propagation analysis. It is based on the TOUGH2 simulator for non-isothermal multiphase flow in fractured and porous media [Pruess, 1987, 1991a]. Extensive experience in using TOUGH2 is a prerequisite for using iTOUGH2. The preparation of an input file for TOUGH2 or its derivatives is described in separate manuals and is not part of this report.

The "iTOUGH2 User's Guide" [Finsterle, 1999a] summarizes the inverse modeling theory pertaining to iTOUGH2, and describes the program output. Furthermore, information about code architecture and installation are given. In Chapter 2 of this report, a brief summary of inverse modeling theory is given to restate the main concepts implemented in iTOUGH2 and to introduce certain definitions. Chapter 3 introduces the basic concepts of the iTOUGH2 input language and the main structure of an iTOUGH2 input file. Chapter 4, the main part of this report, provides detailed descriptions of each iTOUGH2 command in alphabetical order. It is complemented by a command index in Appendix B in which the commands are given in logical order. The content of Chapter 4 is also available on-line using command it 2 help. Chapter 5 describes the usage of the UNIX script files for executing, checking, and terminating iTOUGH2 simulations.

A variety of inverse problems solved by iTOUGH2 are discussed in a collection of sample problems [Finsterle, 1999c], which includes a tutorial example illustrating the main features of iTOUGH2. Complete examples of iTOUGH2 input files are given along with interpretations of the corresponding output files.

The key to a successful application of iTOUGH2 is (i) a good understanding of multiphase flow processes, (ii) the ability to conceptualize the given flow and transport problem and to develop a corresponding TOUGH2 model, (iii) detailed knowledge about the data used for calibration, (iv) an understanding of parameter estimation theory and the correct interpretation of inverse modeling results, (v) proficiency in using iTOUGH2 options. This report addresses issue (v) only. 


\section{ITOUGH2 SUMMARY DESCRIPTION}

A comprehensive description of inverse modeling theory implemented in iTOUGH2 is given in Finsterle [1999a]. The purpose of the summary description provided here is mainly to introduce basic concepts and notations referred to in Chapter 4 of this report.

iTOUGH2 is a computer program that provides inverse modeling capabilities for the TOUGH 2 code. While the main purpose of iTOUGH2 is to estimate model-related hydraulic properties by calibrating TOUGH2 models to laboratory or field data, the information obtained by evaluating parameter sensitivities can be used to scrutinize the design of an experiment and to evaluate the uncertainty of model predictions.

iTOUGH2 solves the inverse problem by automatic model calibration. All TOUGH2 input parameters can be considered unknown or uncertain. The parameters are estimated based on any type of observation for which a corresponding TOUGH2 output is available, including prior information about the parameters to be estimated. A number of different objective functions and minimization algorithms are available. One of the key features of iTOUGH2 is its extensive error analysis which provides statistical information about residuals, estimation uncertainties, and the ability to discriminate among model alternatives. The impact of parameter uncertainties on model predictions can be studied by means of linear uncertainty propagation analysis or Monte Carlo simulations.

The key elements of an inverse modeling code are (1) a simulation program to model flow and transport in the hydrogeologic system ("forward modeling"), (2) the objective function which measures the misfit between the model output and the data, (3) the minimization algorithm which reduces the objective function by automatically updating parameter values, and (4) the error analysis which allows one to judge the quality of the estimates.

iTOUGH2 estimates elements of a parameter vector $\mathbf{p}$ based on observations summarized in vector $\mathbf{z}^{*}$ by minimizing an objective function $S$ which is a function of the residual vector $\mathbf{r}$.

Vector $\mathbf{p}$ of length $n$ contains the parameters to be estimated by inverse modeling, or the parameters considered uncertain for uncertainty propagation analysis.

$$
\text { Parameter vector: } \quad \mathbf{p}^{T}=\left[p_{1}, p_{2}, \ldots, p_{n}\right]
$$

In the simplest case, the parameter $p$ to be estimated is identical with a TOUGH2 input parameter $X$. For example, $p$ is the porosity of a certain rock type, or a parameter of the capillary pressure function. Other parameters include initial and boundary conditions, or geometrical features such as fracture spacing. iTOUGH2 also allows one to estimate a single parameter which will be assigned to multiple TOUGH2 input variables, i.e., $X$ can represent more than one TOUGH 2 variable. Moreover, user-specified parameters can be programmed into iTOUGH2. 
Simple parameter transformations can be employed to make the inverse problem more linear or to change the distributional assumption about the parameter. For example, the log-normal character of permeability suggests estimating the logarithm of permeability rather than permeability itself. While the transformation does not change the best estimate parameter set, it makes the detection of the corresponding minimum of the objective function more robust. Different results are obtained, however, when incorporating prior information or performing uncertainty propagation analysis. In both cases a prior standard deviation must be given which represents either a normal or log-normal distribution, respectively.

Table 2.1 shows the parameter transformations available in iTOUGH2. Here, $X$ is one or more TOUGH2 input variables (with initial guess $X_{0}$ ), and $p$ is the parameter estimated by iTOUGH2. It is important to realize that the standard deviation, parameter variation, acceptable parameter range, and the starting point for the optimization refer to $p$ (and not $X$ ).

Table 2.1. Parameter Transformations

\begin{tabular}{lll}
\hline$X \rightarrow p$ & $p \rightarrow X$ & iTOUGH2 command \\
\hline$p=X$ & $X=p$ & $>>>$ VALUE \\
$p=\log _{10}(X)$ & $X=10^{p}$ & $>>>$ LOGARITHM \\
$p=X / X_{0}$ & $X=p \cdot X_{0}$ & $>>>$ FACTOR \\
$p=\log _{10}\left(X / X_{0}\right)$ & $X=10^{p} \cdot X_{0}$ & $>>>>$ LOG $(\mathrm{F})$ \\
\hline
\end{tabular}

Vector $\mathbf{z}$ of length $m$ contains dependent, observable variables, usually related to measurements taken at discrete points in space $\mathbf{x}$ and time $t$. Such a selected point in space and time, $z_{i}\left(\mathbf{x}_{j}, t_{k}\right)$, will be referred to as a calibration point. Elements of $\mathbf{z}$ refer to both measured quantities (data), indicated by an asterisk (*), and simulation results. The most commonly used observations for calibration are pressure, flow rate, temperature and concentration measurements.

$$
\text { Observation vector: } \mathbf{z}^{T}=\mathbf{z}\left(\mathbf{x}_{j}, t_{k}\right)^{T}=\left[z_{1}, \ldots, z_{n}, z_{n+1}, \ldots z_{m}\right]
$$

The vector of observable variables may also contain measured parameter values. For example, if permeability has been measured on cores in the laboratory, this information can be considered as an additional data point, and treated along with the direct observations of the system response. Such measured parameter values are referred to as "prior information". The first $n$ elements of $\mathbf{z}$ are therefore identical to the variables of $\mathbf{p}$.

The residual vector $\mathbf{r}$ contains the differences between the measured and calculated system response; the latter is a function of parameter vector $\mathbf{p}$ :

$$
\text { Residual vector: } \quad \mathbf{r}^{T}=\left(\mathbf{z}^{*}-\mathbf{z}(\mathbf{p})\right)^{T}=\left[r_{1}, \ldots, r_{n}, r_{n+1}, \ldots, r_{m}\right]
$$


For example, element $r_{i} \quad(i>n)$ is the difference between the measured and calculated pressure at a certain point in space and time. A special type of residual (for $i \leq n$ ) is the difference between the measured parameter $p_{i} *$ (prior information, if available) and the estimated parameter value.

The elements of vectors $\mathbf{z}$ and $\mathbf{r}$ may have different physical meanings with different units of measurement, and represent observations of different accuracy. The inverse of the covariance matrix of $\mathbf{z}$ can be used as a scaling matrix. If taking the view of maximum likelihood estimation, the covariance matrix is part of the stochastic model, i.e., it represents the distributional assumption about the measurement errors. A reasonable assumption about the measurement errors is that they are a result of many individual error sources, and are thus uncorrelated, normally distributed random variables with zero mean. The distributional assumption can therefore be summarized in a covariance matrix $\mathbf{C}_{z z}$, an $m \times m$ diagonal matrix. The $i$-th diagonal element of matrix $\mathbf{C}_{z z}$ is the variance $\sigma_{i}^{2}$ representing the measurement error of observation $z_{i}$ :

$$
\mathbf{C}_{z z}=\left[\begin{array}{cccccc}
\sigma_{1}^{2} & 0 & 0 & 0 & \cdots & 0 \\
0 & \sigma_{i}^{2} & 0 & 0 & \cdots & 0 \\
0 & 0 & \sigma_{n}^{2} & 0 & \cdots & 0 \\
0 & 0 & 0 & \sigma_{j}^{2} & \cdots & 0 \\
\vdots & \vdots & \vdots & \vdots & \ddots & \vdots \\
0 & 0 & 0 & 0 & \cdots & \sigma_{m}^{2}
\end{array}\right]
$$

The first $n$ diagonal elements are the variances of the prior information vector $\mathbf{p}^{*}$, followed by $m-n$ variances of the observed system state. Note that only the relative magnitude of the elements of $\mathbf{C}_{z z}$ influences the values of the estimated parameters. We therefore introduce a dimensionless factor $\sigma_{0}^{2}$ which is termed the prior error variance, and a positive definite matrix $\mathbf{V}_{z z}$, where $\mathbf{V}_{z z}^{-1}$ will be used as a weighting matrix:

$$
\mathbf{C}_{z z}=\sigma_{0}^{2} \cdot \mathbf{V}_{z z}
$$

While $\sigma_{0}^{2}$ can assume any positive number, it is convenient to set $\sigma_{0}^{2}=1$, i.e., the weighting matrix is the inverse of the covariance matrix.

After the inversion, the a posteriori or estimated error variance $s_{0}^{2}$ is calculated (see Eq. 2.10 below). If the preconception about the measurement errors is correct, and if the true system response is identified, the ratio $\mathrm{s}_{0}^{2} / \sigma_{0}^{2}$ should not significantly deviate from 1.0.

The objective function $S$ is a measure of the misfit between the data and the model calculation. If the measurement errors $\left(\mathbf{z}^{*}-\mathbf{z}\right)$ are normally distributed with mean 
$E\left[\left(\mathbf{z}^{*}-\mathbf{z}\right)\right]=\mathbf{0}$ and covariance matrix $E\left[\left(\mathbf{z}^{*}-\mathbf{z}\right)\left(\mathbf{z}^{*}-\mathbf{z}\right)^{T}\right]=\mathbf{C}_{z z}$, maximum-likelihood theory yields the weighted least-squares objective function:

$$
S=\left(\mathbf{z}^{*}-\mathbf{z}\right)^{T} \mathbf{C}_{z z}^{-1}\left(\mathbf{z}^{*}-\mathbf{z}\right)=\mathbf{r}^{T} \mathbf{C}_{z z}^{-1} \mathbf{r}=\sum_{i=1}^{m} \frac{r_{i}^{2}}{\sigma_{i}^{2}}
$$

Alternative objective functions are available in iTOUGH2 (see Finsterle [1999a]). The best estimate parameter set minimizes the objective function (2.6). Minimization of the objective function $S$ is based on local linearization (see Levenberg-Marquardt algorithm below). The partial derivatives of the calculated system response with respect to the parameters are summarized in the Jacobian matrix $\mathbf{J}$ of dimensions $m \times n$, the elements of which are defined as follows:

$$
J_{i j}=-\frac{\partial r_{i}}{\partial p_{j}}=\frac{\partial z_{i}}{\partial p_{j}}
$$

iTOUGH2 calculates $\mathbf{J}$ numerically by means of either forward or centered finite differences. The Jacobian is also important in the a posteriori, linear error analysis as well as the uncertainty propagation analysis.

While iTOUGH2 offers a number of different minimization algorithms, the LevenbergMarquardt modification of the Gauss-Newton algorithm is found to be a rather general and robust procedure for iteratively updating parameter vector $\mathbf{p}$ [Levenberg, 1944; Marquardt, 1963]. It can be described as shown in Table 2.2. If $\lambda$ is large, the first term on the righthand side of (2.8) becomes a matrix with a dominant diagonal. This leads to a small step along the gradient of $S$. Stepping along the steepest descent direction is robust, but inefficient. The Levenberg parameter $\lambda$ is decreased after each successful step. With decreasing $\lambda$, the parameter update $\Delta \mathbf{p}$ (Eq. 2.8) converges to the one proposed by the Gauss-Newton algorithm with its quadratic convergence rate. 
Table 2.2. Levenberg-Marquardt Minimization Algorithm

Step 1: Initialization:

- Set iteration index $k=0$.

- Define initial Levenberg parameter (default: $\lambda_{0}=10^{-3}$ ).

- Define Marquardt parameter (default: $v=10$ ).

- Define initial parameter set $\mathbf{p}_{k=0}=\mathbf{p}_{0}$.

Step 2: Run simulation model with parameter vector $\mathbf{p}_{k}$.

Step 3: Evaluate $\mathbf{r}\left(\mathbf{p}_{k}\right), S\left(\mathbf{p}_{k}\right)$, and $\mathbf{J}\left(\mathbf{p}_{k}\right)$.

Step 4: Calculate parameter update: $\Delta \mathbf{p}_{k}=\left(\mathbf{J}_{k}^{T} \mathbf{C}_{z z}^{-1} \mathbf{J}_{k}+\lambda_{k} \mathbf{D}_{k}\right)^{-1} \mathbf{J}_{k}^{T} \mathbf{C}_{z z}^{-1} \mathbf{r}_{k}$

where $\mathbf{D}_{k}$ is an $n \times n$ matrix with elements $D_{j j}=\left(\mathbf{J}_{k}^{T} \mathbf{C}_{z z}^{-1} \mathbf{J}_{k}\right)_{j j}, j=1, \ldots, n$.

Step 5: Update parameter vector: $\mathbf{p}_{k+1}=\mathbf{p}_{k}+\Delta \mathbf{p}_{k}$.

Step 6: Perform simulation and evaluate $S\left(\mathbf{p}_{k+1}\right)$.

Step 7: If $S\left(\mathbf{p}_{k+1}\right)<S\left(\mathbf{p}_{k}\right)$ multiply $\lambda$ by factor $1 / v$ and go to Step 8 .

If $S\left(\mathbf{p}_{k+1}\right)>S\left(\mathbf{p}_{k}\right)$ multiply $\lambda$ by factor $v$ and go to Step 4 .

Step 8: Evaluate convergence criteria.

If converged, go to Step 9, else set $k=k+1$ and go to Step 2 .

Step 9: Minimum identified. Proceed with residual and uncertainty analysis.

One of the key advantages of a formalized approach to parameter estimation is the possibility to perform an extensive a posteriori error analysis. First, the residual analysis provides some measure of the overall goodness-of-fit and allows identification of systematic errors or flaws in the stochastic model. Next we can determine the uncertainty of the estimated parameters. Note that a good match does not necessarily mean that the estimates are reasonable. They may be highly uncertain due to a lack of sensitivity or high parameter correlations, which is an indication that the inverse problem is over-parameterized. The covariance matrix of the estimated parameters can be further analyzed to obtain correlation coefficients, indicating parameter combinations that lead to similar matches, etc. Finally, we can calculate the uncertainty of the simulation results, which also allows us to identify potential outliers in the data. 
The estimated error variance $s_{0}^{2}$ represents the variance of the weighted residuals and is thus a measure of goodness-of-fit:

$$
s_{0}^{2}=\frac{\mathbf{r}^{T} \mathbf{V}_{z z}^{-1} \mathbf{r}}{m-n}
$$

Note that if the residuals are consistent with the distributional assumption about the measurement errors (i.e., matrix $\mathbf{C}_{z z}$ ), then the estimated error variance assumes a value close to $\sigma_{0}^{2}$. Next we calculate the expected value and the covariance matrix $\mathbf{C}_{p p}$ of the estimated parameters. Based on the linearity assumption it is easy to show that the expected value of the estimated parameter is equal to the parameter itself. The definition of the covariance matrix yields:

$$
\mathbf{C}_{p p}=s_{0}^{2}\left(\mathbf{J}^{T} \mathbf{V}_{z z}^{-1} \mathbf{J}\right)^{-1}
$$

The interpretation of the covariance matrix $\mathbf{C}_{p p}$ provides the key criteria to evaluate the inverse modeling results.

This concludes the summary description of iTOUGH2. Again, more details about inverse modeling theory, objective functions, minimization algorithms, and the residual and error analysis can be found in Finsterle [1999a]. 


\section{BASIC CONCEPTS}

\subsection{Formats of iTOUGH2 Input Files}

The user must provide at least two input files to run iTOUGH2. The first one is a TOUGH2 input file in standard TOUGH2 format as described in Pruess [1987, 1991a] and Falta et al. [1995], as well as other publications pertaining to particular TOUGH2 modules and code enhancements (e.g., Pruess [1991b], Finsterle et al. [1994], Moridis and Pruess [1995]). This input file defines the conceptual model. It solves the forward problem and should run successfully using standard TOUGH2 not only for the initial parameter set, but also for a wide range of potential parameter combinations.

The second input file is the iTOUGH2 input file, in which the user specifies the parameters to be estimated, the observations used for calibration, and various program options (see Section 3.3). A special command interpreter has been developed to read the iTOUGH2 input file. The basic concept of the iTOUGH2 input language is discussed in Section 3.2. A detailed description of each iTOUGH2 command is given in Chapter 4.

\subsection{Basic Concepts of iTOUGH2 Input Language}

Execution of iTOUGH2 is controlled by means of a high-level input language. The commands are hierarchically structured, i.e., each command has a parent command on a higher command level, and potentially one or more child commands on a lower command level. The command level is identified by the corresponding number of "> markers (e.g., ">>>" to enter command level three). Each command level must be terminated by reversed markers (e.g., " $<<<"$ to quit command level three and return to level two). The position of the command level marker on a line is irrelevant. Any lines not containing a command level marker are considered to be a comment and are skipped, unless they represent data requested by a previous command. Entire sections of the input file may be commented out by marking the first and last line with $/ *$ and $\star /$, respectively. All lines between these two marks are not interpreted even if they contain command level markers.

On the line containing a command level marker, a command is expected which is unique to the corresponding command level and the associated parent command. A command can be complemented by optional keywords. Additional text present on the command line is ignored. Commands and keywords are case-insensitive. The position of the command and keywords on a command line is arbitrary. Many commands request additional numerical or alphanumeric input. This input can be provided anywhere on the command line, but must immediately follow a colon ":". Input may consist of one or more integers, real numbers, or strings. Input belonging to the same command can be continued on following lines by using the " \&" character. Each line of the input file is processed word by word, the delimiter being one or more spaces. All numerical data are read in free format. Long lists of data start one line after the command line. Data lists will be read until a FORTRAN reading error occurs. 
Command LIST can be used on any command level to obtain a list of acceptable commands on the current command level. The keyword HELP or MANUAL can be used on any command line to print a short tutorial of the corresponding command to the output file.

In general, subcommands of the same parent command can be provided in arbitrary order. A few exceptions apply; they are clearly identified when using an incorrect order.

Examples:
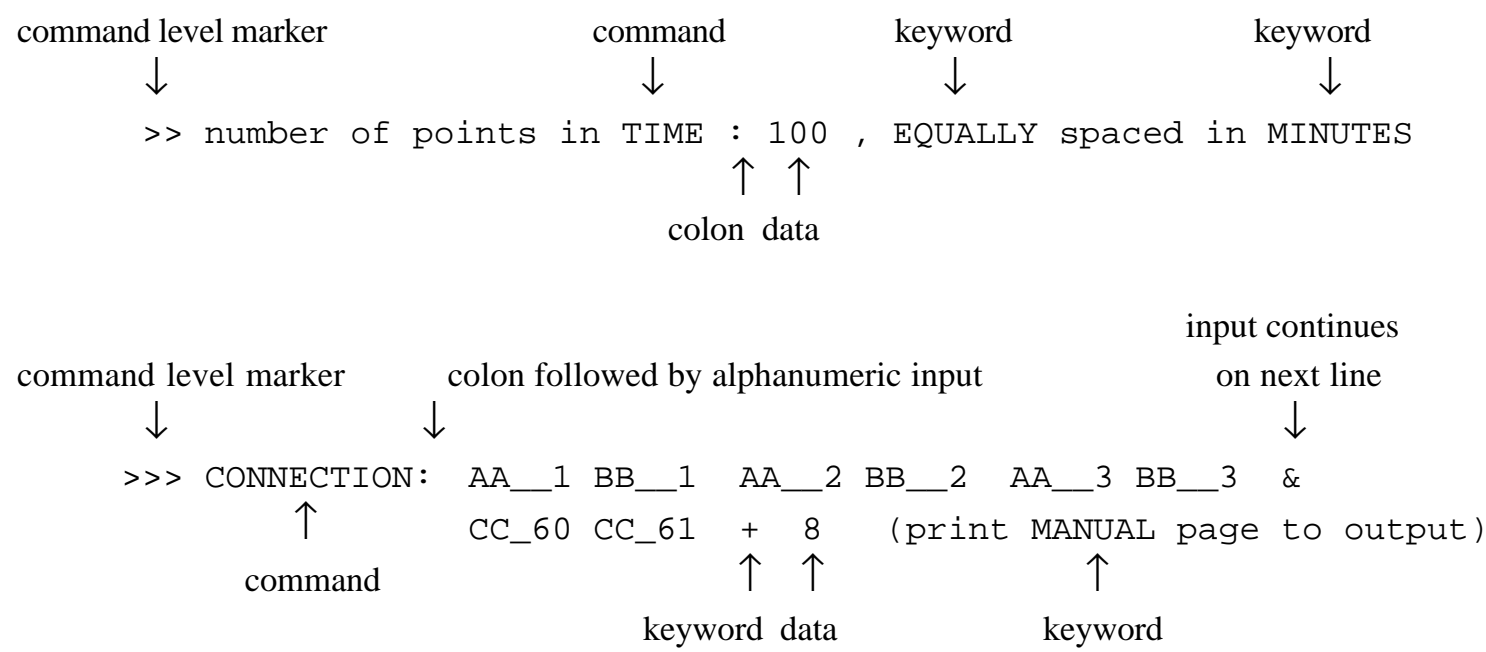

\subsection{Main Structure of iTOUGH2 Input}

There are three main blocks in the iTOUGH2 input file, identified by one of the following first-level commands:

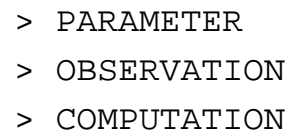

The first block (first-level command > PARAMETER) is used to identify those TOUGH2 input parameters that will be subjected to parameter estimation, sensitivity analysis, or uncertainty propagation analysis. A series of subcommands ensure a unique identification of the parameter by providing its type (second-level commands), and the domain it is referring to (third-level commands). The fourth-level commands are used to provide further specifications along with statistical details about the parameter.

The second block (first-level command > OBSERVATION) is used to identify those TOUGH2 output variables that will be compared to observed data for model calibration. Furthermore, the points in time at which calibration should occur are also specified in this block. An observable variable is identified by its type (second-level command), its location 
(third-level command), as well as more detailed specifications if necessary (fourth-level commands). The measured data to which the calculated system response is compared to is also provided through a fourth-level command.

The third block (first-level command > COMPUTATION) is used for various program options and computational parameters. It deals with convergence criteria, the numerical calculation of the Jacobian matrix, statistical parameters for the error analysis, and output options. Most important, the application mode of iTOUGH2 (parameter estimation, sensitivity analysis, uncertainty propagation analysis) can be selected along with a number of additional features.

Figure 3.3.1 depicts the main structure of an iTOUGH2 input file in a generic way. A list of available subcommands can be found in Appendix B, and a detailed description of each iTOUGH2 command is given in Chapter 4.

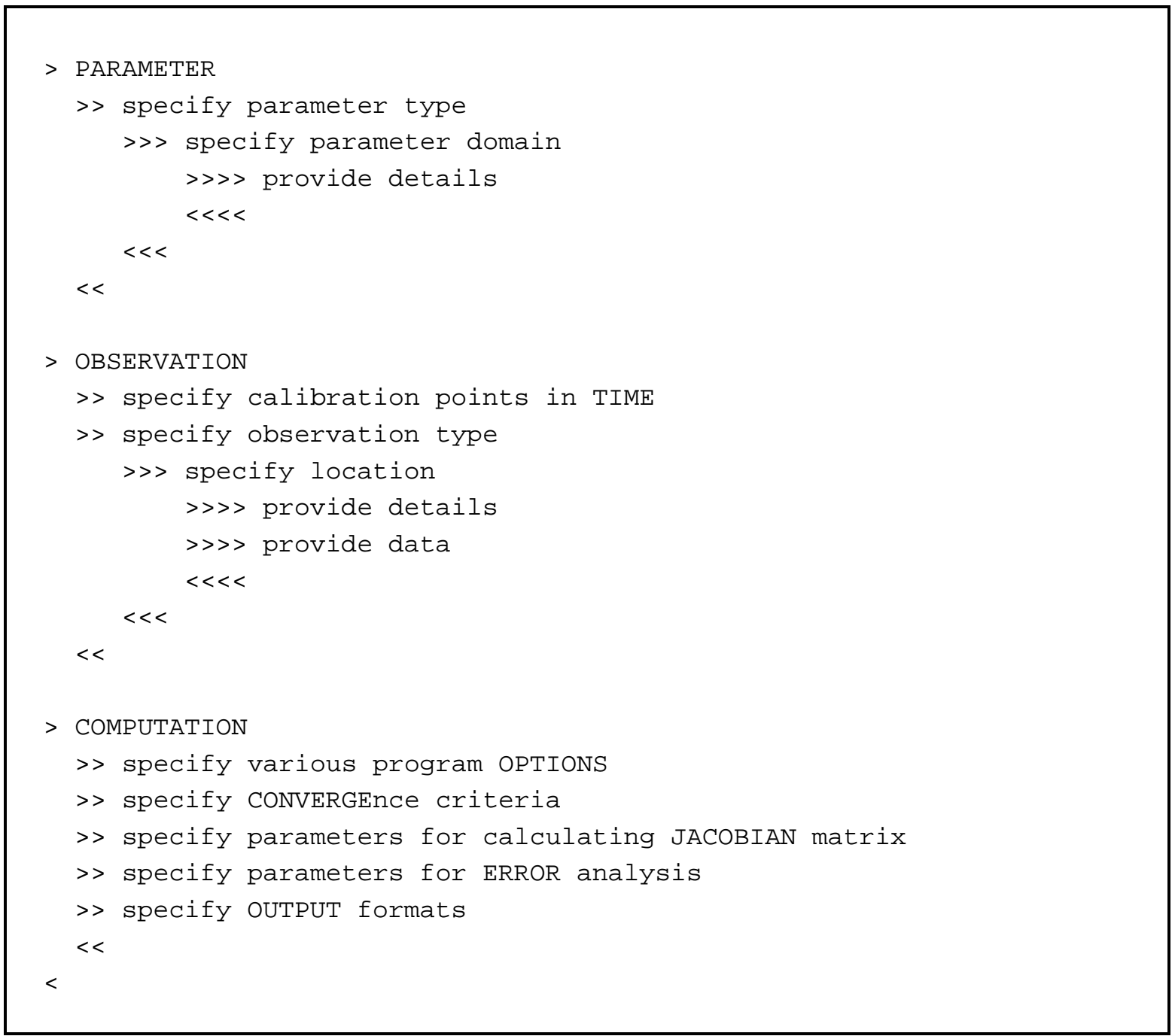

Figure 3.3.1. Main structure of iTOUGH2 input. 


\section{ITOUGH2 COMMANDS}

A detailed description of each iTOUGH2 command is given below. The description includes the command syntax, the name of the parent command, the name of subcommands (if applicable), as well as the purpose and effect of the command. Theoretical background is provided where necessary. Furthermore, an illustrative example is given for each command.

In general, the command syntax includes the command level marker, the command name as identified by iTOUGH2, input variables (in italics), and optional keywords or variables (in parentheses). Alternatives are separated by a slash "/".

Example:

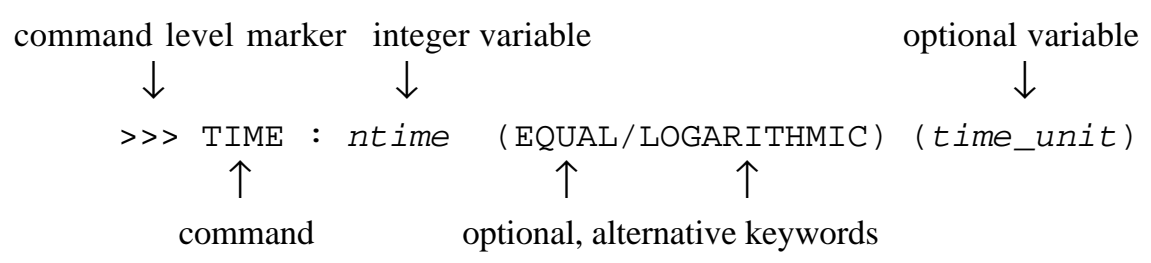

The commands are given in alphabetic order. Note that the name of a command is not always unique, in which case the command level and the parent command can be used for identification. If reference is made to a command with a non-unique command name, the first character of the parent command (or the corresponding first-level command) is indicated in parentheses (e.g., $>>>$ FORWARD (c) refers to the command in block $>>$ CONVERGE, whereas >> FORWARD $(j)$ refers to the command in block $>$ JACOBIAN).

iTOUGH2 commands can be written in lower or upper case. In this manual, the commands and keywords are always typed in upper case for easy identification. Therefore, the lower case words on the command line are comments that will be ignored by the iTOUGH2 command interpreter.

The examples given for each command usually illustrate the basic usage of a command. In many cases, however, extended features and advanced applications are provided to demonstrate the use of a command in combination with other commands. The examples are not complete and can therefore not be run separately. Furthermore, they may have to be modified to avoid warning messages.

The command description can also be accessed online by typing it 2 help, which is the name of the UNIX script file it 2 help. 


\section{Command}

>> ABSOLUTE

\section{Parent Command}

$>$ PARAMETER

\section{Subcommand}

>> MATERIAL

\section{Description}

This command selects as a parameter the absolute permeability (TOUGH2 variable PER (ISOT, NMAT)) of the specified material.

Subcommand $>>>>$ LOGARITHM invokes estimation of a single log-value which is assigned to all selected materials; subcommand $>>>>$ FACTOR invokes estimation of a common multiplication factor which is applied to all selected permeabilities, thus maintaining the permeability ratios between the materials; subcommand $>>>>$ LOG $(F)$ should be used to estimate a log-normally distributed factor with which all the permeabilities are multiplied. By default, the estimate refers to all three flow directions ( ISOT=1, 2, 3). Subcommand $>>>$ INDEX can be used to select the permeability of a specific flow direction ISOT.

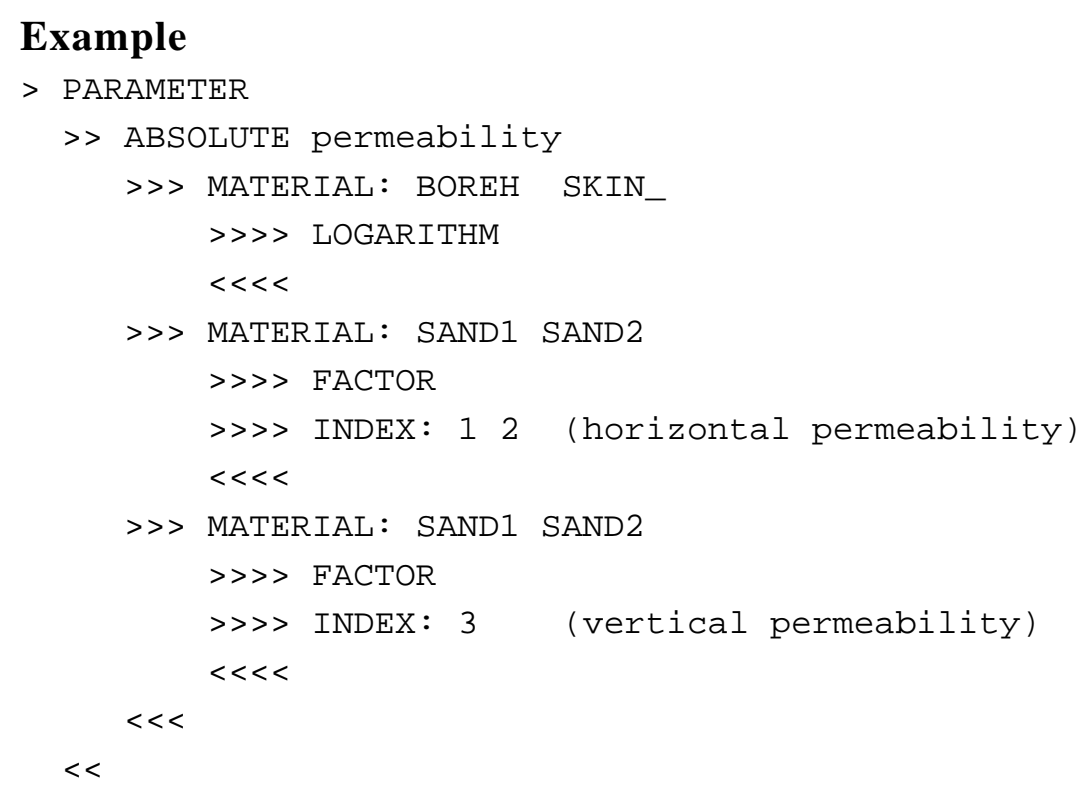

\section{See Also}




\section{Command}

>>> ABSOLUTE

\section{Parent Command}

all third-level commands in block > OBSERVATION

\section{Subcommand}

$-$

\section{Description}

This command takes the absolute value of the calculated system response as the model output to be compared to the observed data.

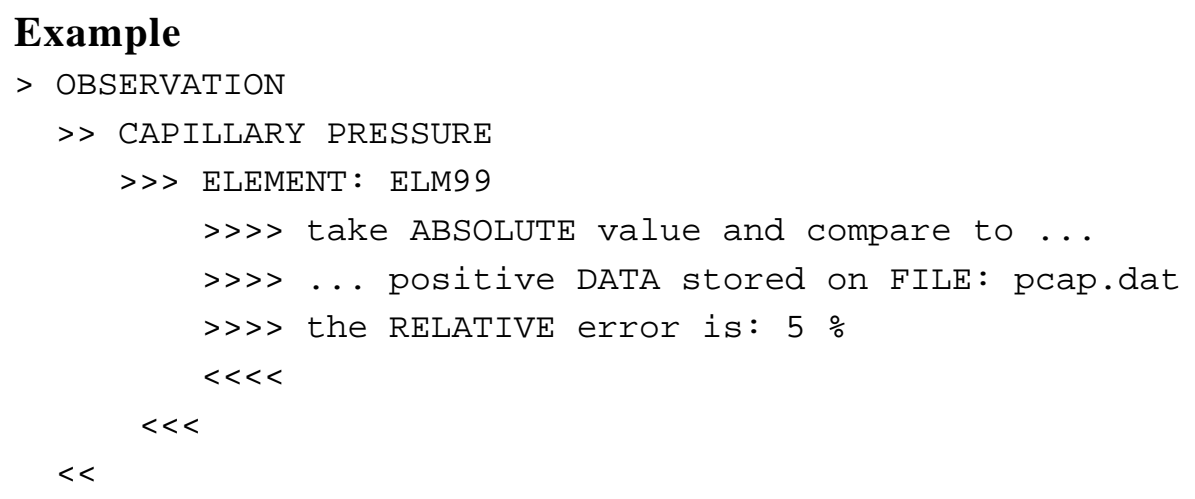

See Also 


\section{Command}

$>>$ ADJUST

Parent Command

>> CONVERGE

\section{Subcommand}

$-$

\section{Description}

The initial time step size is provided through TOUGH2 variable DELTEN or DLT (1), usually followed by automatic time stepping (see TOUGH2 variables MOP (16) and $R E D L T$ ). The initial time step may be too big (i.e., is automatically reduced by TOUGH2) or too small (i.e., convergence is achieved within one Newton-Raphson iteration), depending on the parameter set supplied by iTOUGH2 during an inversion. This command allows iTOUGH2 to automatically adjust the initial time step size so that convergence is achieved within more than 1 but less than $M O P(16)$ Newton-Raphson iterations. Automatic time step adjustment may improve the speed of an inversion, but may also make the inversion unstable if time discretization errors are significant.

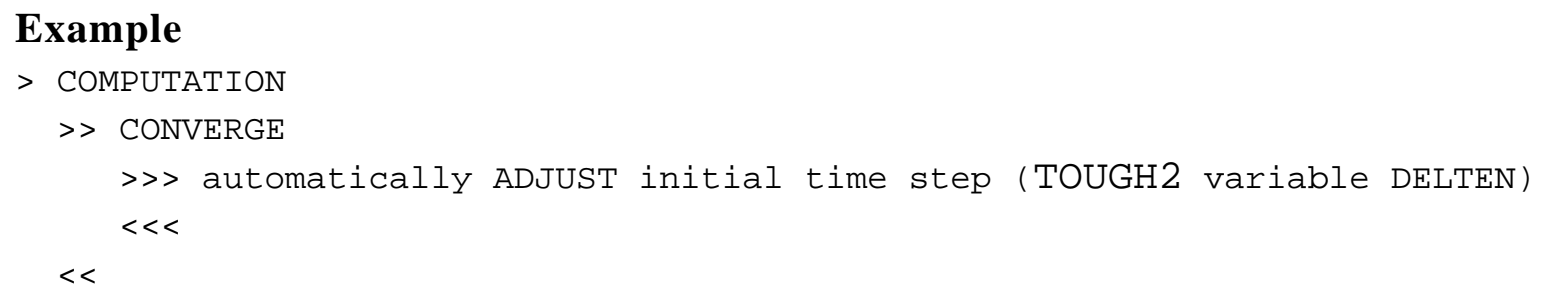

See Also

>> CONSECUTIVE, >>> REDUCTION 


\section{Command}

>> ALPHA: alpha (\%)

\section{Parent Command}

$>$ ERROR

\section{Subcommand}

$-$

\section{Description}

alpha is a probability used for a variety of statistical tests within iTOUGH2. The choice of alpha does not affect the estimated parameter set, but alpha is used in the residual analysis, the Fisher model test, the analysis of the resulting distribution when performing Monte Carlo simulations, and the width of the error band when performing FOSM uncertainty propagation analysis. alpha is expected to assume values between 0.001 and 0.200 (default: 0.01). Instead of the risk $\alpha$, one can also provide the confidence level $(1-\alpha)$, in which case alpha assumes values between 0.8 an 0.999 . Use keyword $\%$ if alpha is given in percent.

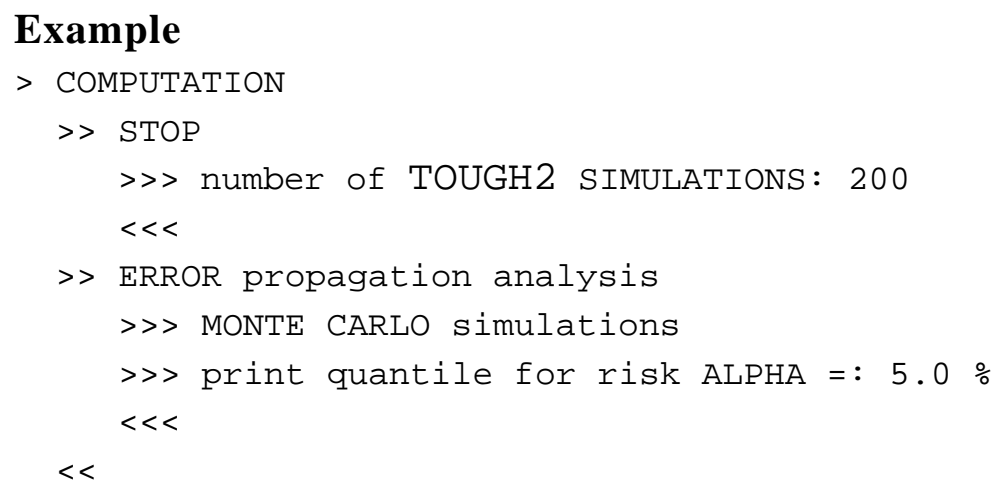

See Also

$-$ 


\section{Command}

>> ANDREWS: $C$

\section{Parent Command}

>> OPTION

\section{Subcommand}

$-$

\section{Description}

This command selects a robust estimator named Andrews. Given this estimator, the objective function to be minimized is the sum of the cosine functions $\gamma\left(y_{i}\right)$, where $y_{i}$ is the weighted residual:

$$
S=\sum_{i=1}^{m} \gamma\left(y_{i}\right)
$$

where

$$
\gamma\left(y_{i}\right)=\left\{\begin{array}{cc}
1-\cos \left(y_{i} / c\right) & \left|\begin{array}{l}
y_{i} \\
y_{i}
\end{array}\right| \leq c \pi \\
2 & >c \pi
\end{array}\right.
$$

with

$$
y_{i}=\frac{r_{i}}{\sigma_{i}}
$$

This objective function does not correspond to a standard probability density function. It has the general characteristic that the weight given individual residuals first increases with deviation, then decreases to reduce the impact of outliers. The parameter $c$ indicates the deviation at which residuals are considered to be outliers. If the measurement errors happen to be close to a normal distribution with standard deviation $\sigma_{i}$, then the optimal value for the constant $c$ is $c=2.1$.

Note that this objective function is minimized using the standard Levenberg-Marquardt algorithm which is designed for a quadratic objective function. Since (1- cos) can be reasonably well approximated by a quadratic function for small $y_{i}$, the Levenberg-Marquardt algorithm is usually quite efficient.

\section{Example}

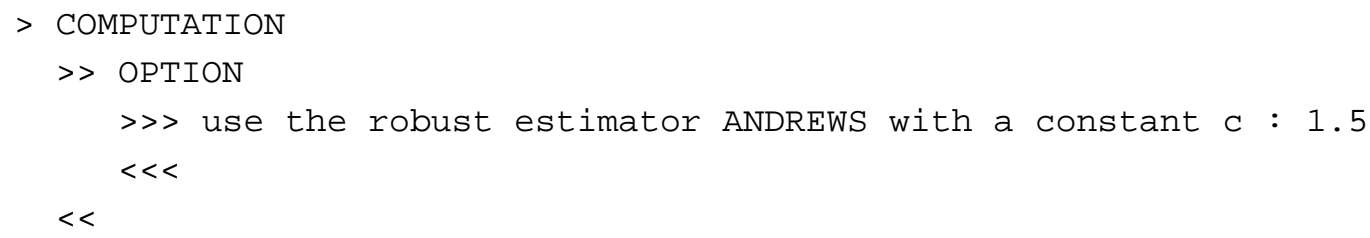

See Also

>>> CAUCHY, >>> L1-ESTIMATOR, >>> LEAST-SQUARES, $>>>$ QUADRATIC-LINEAR 


\section{Command}

$>>$ ANNEAL

\section{Parent Command}

$>>$ OPTION

\section{Subcommand}

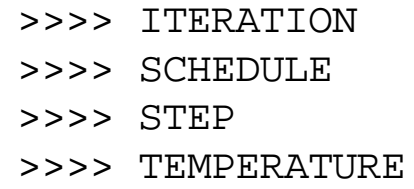

\section{Description}

This command invokes Simulated Annealing to minimize the objective function $S$. The following steps are performed by iTOUGH2, controlled by a number of fourth-level commands:

(1) Define the range of possible parameter values using command $>>>>$ RANGE in block > PARAMETER.

(2) Define an initial value of the control parameter $\tau$ using command $>>>$ TEMPERATURE.

(3) iTOUGH2 generates random perturbations $\Delta \mathbf{p}$ of the parameter vector $\mathbf{p}$. The probability density function of the perturbation is either Gaussian or uniform; the initial standard deviations of these distributions are given by command $>>>$ DEVIATION (p) .

(4) The objective function $S\left(\mathbf{p}_{k+1}\right)$ for the new parameter set $\mathbf{p}_{k+1}=\mathbf{p}_{k}+\Delta \mathbf{p}$ is evaluated.

(5) If the objective function is decreased (i.e., $\left.\Delta S=S\left(\mathbf{p}_{k+1}\right)-S\left(\mathbf{p}_{k}\right)<0\right)$, the change is retained. If the objective function is increased (i.e., $\Delta S>0$ ), the perturbation is accepted with probability $\Pi=\exp (-\Delta S / \tau)$.

(6) After a sufficient number of perturbations have been accepted (see command $>>>$ STEP (a)), $\tau$ is lowered according to the annealing schedule (see command $>>>$ SCHEDULE).

(7) Steps (3) through (6) are repeated until the maximum number of temperature reductions (see command $>>>>$ TRIAL (a)) is reached.

This scheme of always taking a downhill step and sometimes taking an uphill step with probability $\Pi$ depending on $\tau$ is known as the Metropolis algorithm.

Simulated Annealing may be especially useful for the minimization of a discontinuous cost function in order to optimize operational parameters. 


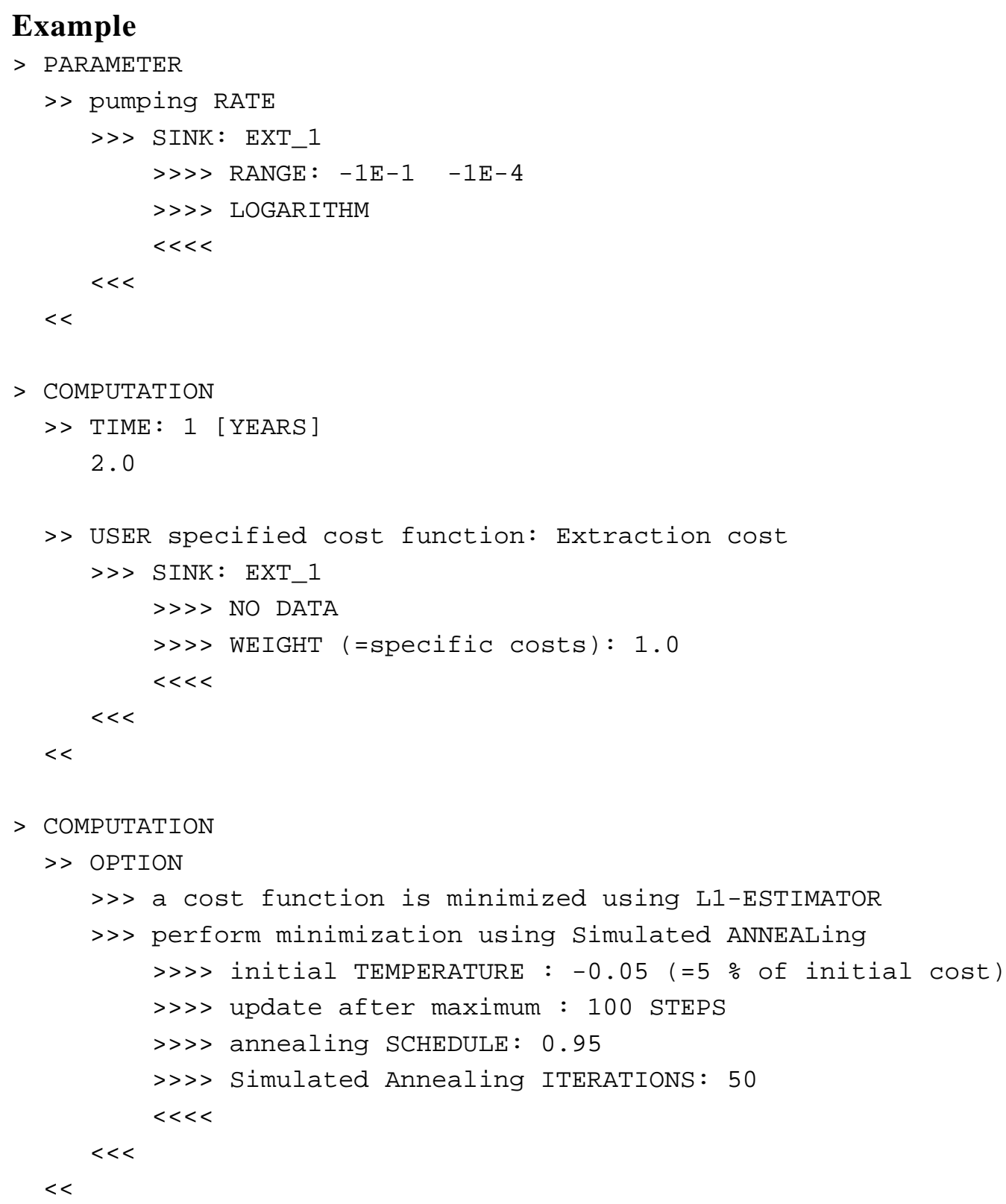

\section{See Also}

>>> GAUSS-NEWTON, >>> GRID SEARCH, >>> LEVENBERG-MARQUARDT, >> SIMPLEX, >>> ITERATION (a), >>>> SCHEDULE, >>> STEP (a), $>>>$ TEMPERATURE 


\section{Command}

>>> ANNOTATION: anno

\section{Parent Command}

all third-level commands in block > PARAMETER and > OBSERVATION

\section{Subcommand}

\section{Description}

A fifteen-character string anno can be provided to label parameters and observations in the iTOUGH2 output file. The annotation does not have any function except for making the iTOUGH2 output more readable (exceptions are the user-specified functions; see commands $>\operatorname{USER}(\mathrm{p}, \mathrm{o})$. If no annotation is provided, iTOUGH2 internally generates a string which allows unique identification of the parameter or observation, respectively. The internally generated annotation can be used to check the correctness of the iTOUGH2 input.

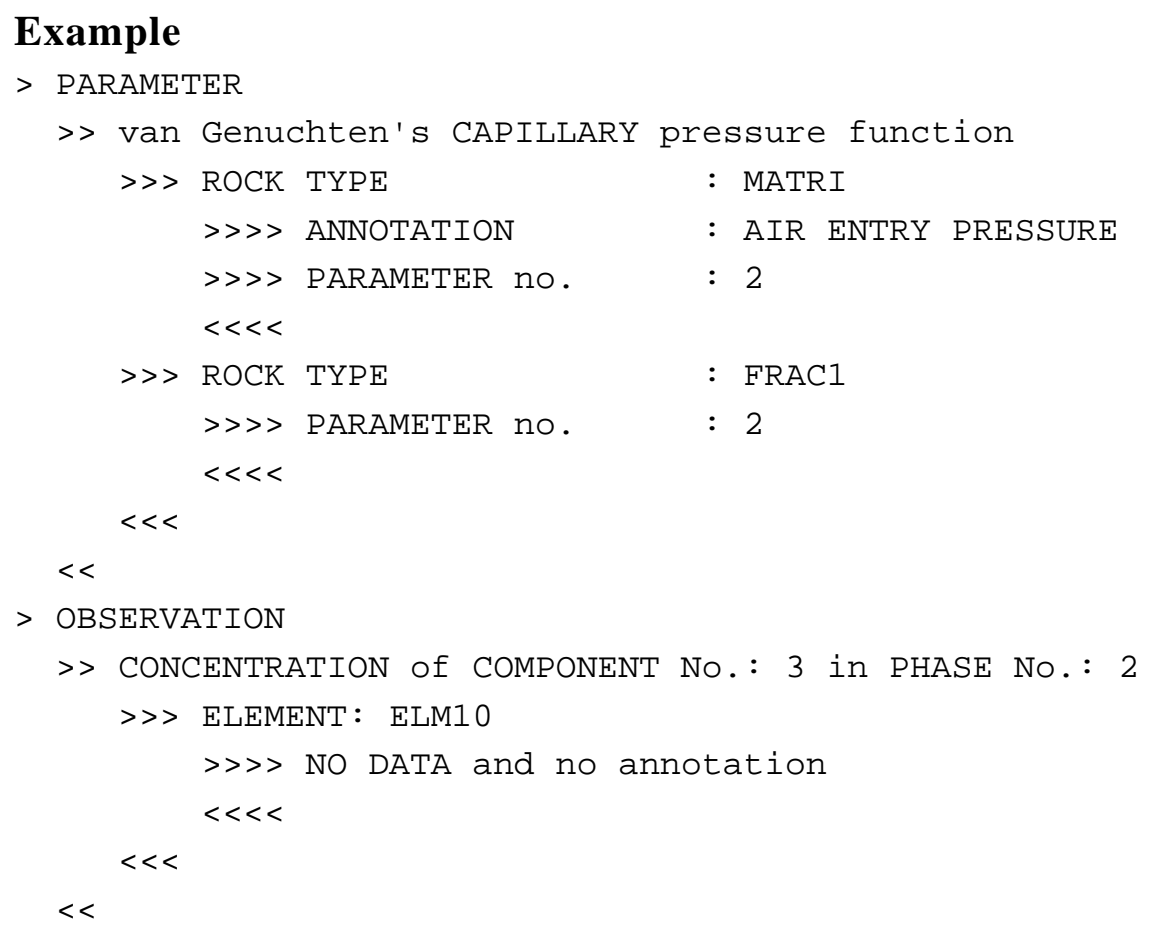

In the iTOUGH2 output file, the first parameter is referred to as "AIR ENTRY PRESS". The annotation internally generated for the second parameter reads "CAP.PR.2 FRAC1", where " 2 " indicates that the second parameter of the capillary pressure ("CAP . PR.") function referring to rock type "FRAC1" is estimated.

The automatically generated annotation for the observation reads "CONC . $(3,2)$ ELM1 0".

\section{See Also}




\section{Command}

$>>>$ AUTO

\section{Parent Command}

all third-level commands in block > OBSERVATION

\section{Subcommand}

$-$

\section{Description}

This command provides automatic weighting of observations. The standard deviation calculated by iTOUGH2 is $10 \%$ of the mean of the observed values for a given data set. It is suggested, however, that the assumed measurement error or expected standard deviation of the final residuals be explicitly provided using command $\gg>>$ DEVIATION (o) .

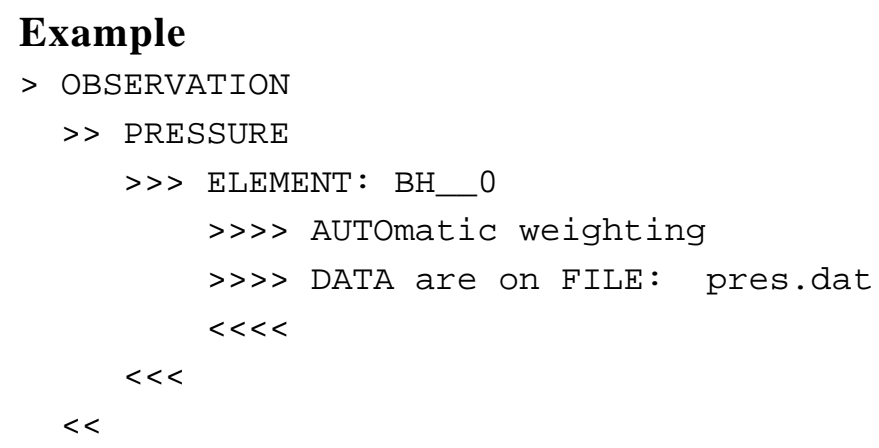

See Also

>>> DEVIATION (O) 


\section{Command}

>>> AVERAGE (VOLUME)

or

>>> MEAN (VOLUME)

Parent Command

all third-level commands in block > OBSERVATION

\section{Subcommand}

$-$

\section{Description}

If multiple elements or connections are provided to indicate the location of a measurement point, iTOUGH2 takes the average of all calculated values as the model output to be compared to the data. The user must ensure that the averaging of the quantity is meaningful. If keyword VOLUME is present on the command line, the calculated values are weighted by the grid block volume.

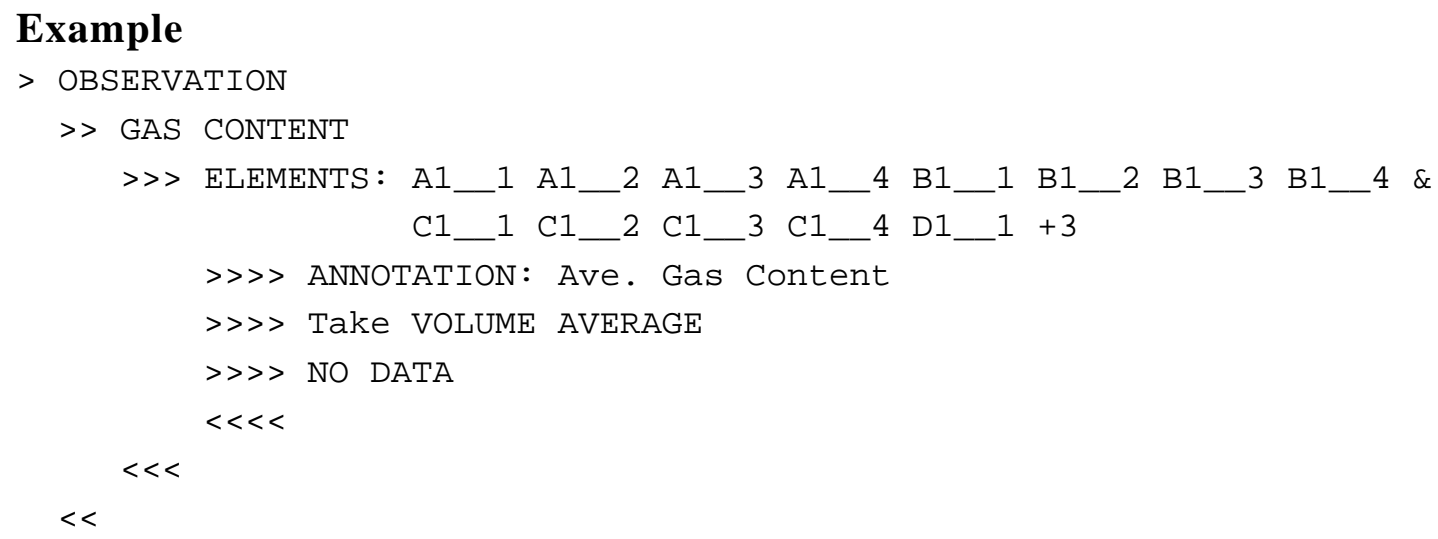

See Also

$>>>$ SUM 


\section{Command}

>> BOTTOMHOLE PRESSURE

\section{Parent Command}

$>$ PARAMETER

\section{Subcommand}

>> SOURCE

\section{Description}

This command selects as a parameter the bottomhole pressure for wells on deliverability (TOUGH2 variable $E X$ ). This parameter refers to a sink/source code name. The generation type must be DELV.

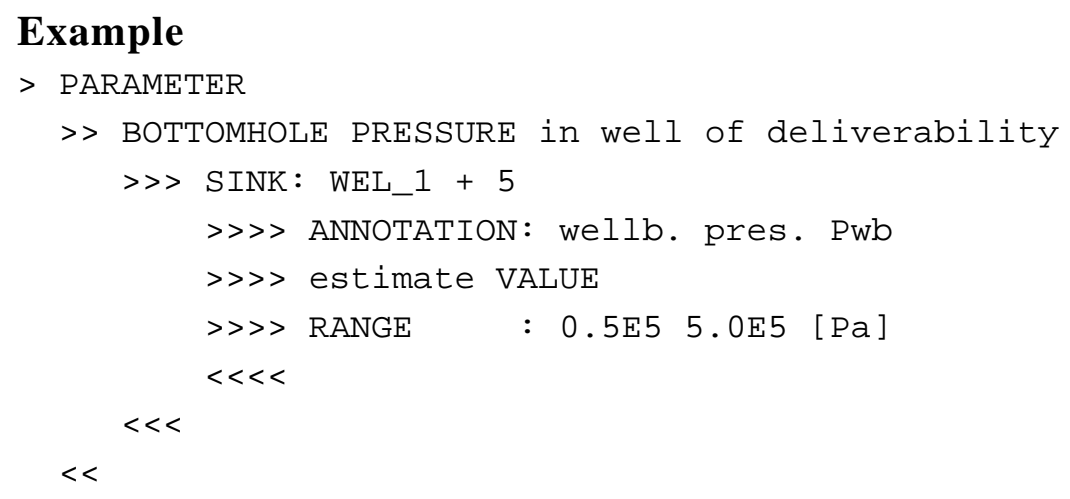

See Also 


\section{Command}

>>> BOUND: lower upper

\section{Parent Command}

all third-level commands in block > PARAMETER

Subcommand

Description

(synonym for command $>>>>$ RANGE)

Example

(see command $>>>>$ RANGE)

See Also

$>>>$ RANGE 


\section{Command}

>> CAPACITY

\section{Parent Command}

$>$ PARAMETER

\section{Subcommand}

>> MATERIAL

\section{Description}

This command selects as a parameter the rock grain specific heat (TOUGH2 variable $\operatorname{SPHT}(N M A T))$.

\section{Example}

$>$ PARAMETER

$>>$ heat CAPACITY

>> ROCK type

$>>>$ VALUE

: GRANI

$>>>$ RANGE

$: 600.03000 .0[\mathrm{~J} / \mathrm{kg} / \mathrm{C}]$

$<<<<$

$<<<$

$<<$

See Also 


\section{Command}

>> CAPILLARY

\section{Parent Command}

$>$ PARAMETER

\section{Subcommand}

$>>>$ DEFAULT

$>>$ MATERIAL

\section{Description}

This command selects a parameter of the capillary pressure function (TOUGH2 variable $C P(I P A R, N M A T))$ of a certain rock type, or a parameter of the default capillary pressure function (TOUGH2 variable CPD (IPAR)). Use command >>> INDEX to select the parameter index IPAR. The physical meaning of the parameter depends on the type of capillary pressure function selected in the TOUGH2 input file, variable ICP and ICPD, respectively. The admissible range should be specified explicitly to comply with parameter restrictions (see Pruess [1987], Appendix B).

\section{Example}

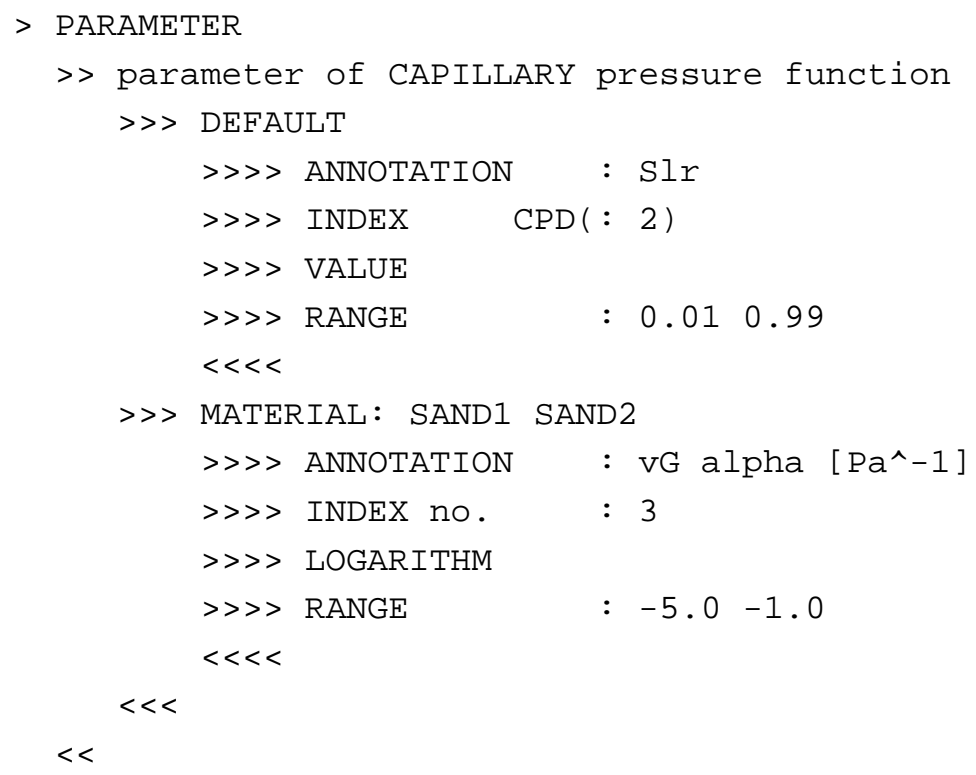

See Also

$>$ RELATIVE 


\section{Command}

$>>$ CAUCHY

\section{Parent Command}

$>>$ OPTION

\section{Subcommand}

$-$

\section{Description}

This command selects an objective function that corresponds to a Cauchy or Lorentzian distribution, i.e., the probability density function of the residuals reads:

$$
\varphi\left(r_{i}\right) \sim \frac{1}{1+\frac{1}{2}\left(\frac{r_{i}}{\sigma_{i}}\right)^{2}}
$$

This distribution exhibits more extensive tails compared to the normal distribution, and leads therefore to a more robust estimation if outliers are present. The objective function to be minimized is given by the following equation:

with

$$
S=\sum_{i=1}^{m} \log \left(1+\frac{1}{2} y_{i}^{2}\right)
$$

$$
y_{i}=\frac{r_{i}}{\sigma_{i}}
$$

This objective function can be minimized using the standard Levenberg-Marquardt algorithm which is designed for a quadratic objective function. The objective function can be reasonably well approximated by a quadratic function, so that the Levenberg-Marquardt algorithm is usually quite efficient.

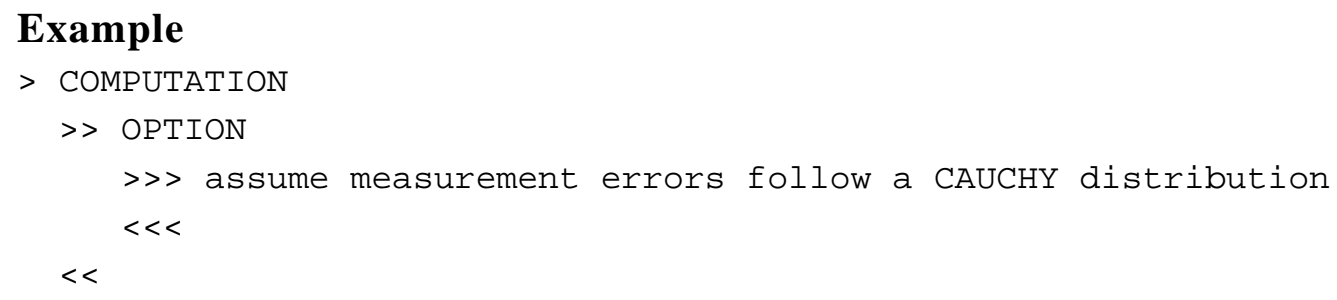

See Also

>> ANDREWS, >>> L1-ESTIMATOR, >>> LEAST-SQUARES, $>>$ QUADRATIC-LINEAR 


\section{Command}

>> CENTERED

\section{Parent Command}

$>>$ JACOBIAN

\section{Subcommand}

$-$

\section{Description}

This command calculates elements of the Jacobian matrix by means of centered finite difference quotients:

$$
J_{i j}=\frac{\partial z_{i}}{\partial p_{j}} \approx \frac{z_{i}\left(\mathbf{p} ; p_{j}+\delta p_{j}\right)-z_{i}\left(\mathbf{p} ; p_{j}-\delta p_{j}\right)}{2 \delta p_{j}}
$$

The evaluation of the Jacobian thus requires $2 n+1$ TOUGH 2 simulations, where $n$ is the number of parameters. The size of the perturbation $\delta p_{j}$ can be controlled using command $>>>$ PERTURB. Centered finite differences are more accurate than forward finite differences, but computationally twice as expensive (also see command $>>>$ FORWARD).

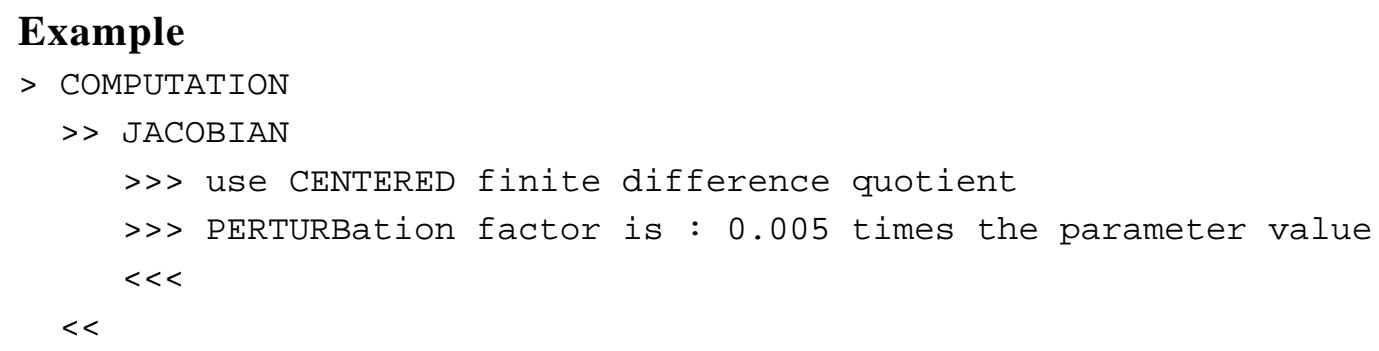

See Also

$>>$ FORWARD $(j),>>>$ PERTURB 


\section{Command}

>> CHARACTERISTIC

\section{Parent Command}

$>$ OUTPUT

\section{Subcommand}

$-$

\section{Description}

This command generates a file in the format specified by command $>>>$ FORMAT containing the characteristic curves (relative permeability and capillary pressure functions) of all rock types used in the TOUGH2 model. The file name contains the string "_ch".

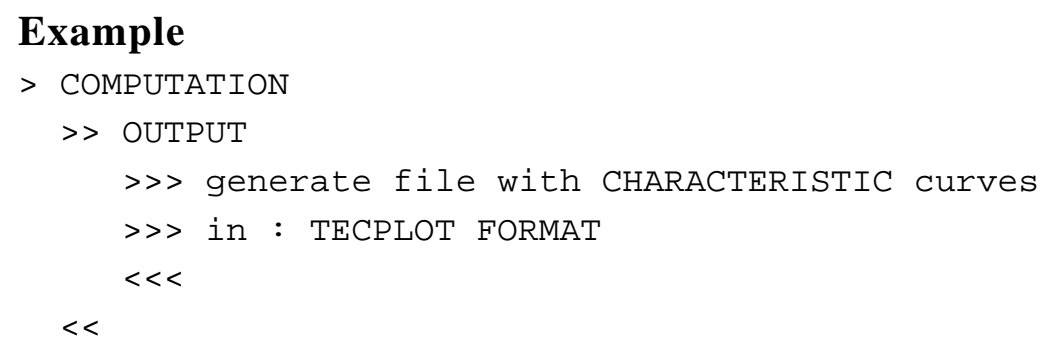

See Also

>> FORMAT 


\section{Command}

>>> COLUMN : itime idata (istd_dev)

Parent Command

all third-level commands in block > OBSERVATION

\section{Subcommand}

\section{Description}

This command identifies the column holding the time and observed value in the data definition block (see command >>> DATA). By default, transient observations are expected to be provided in two columns, where the first column contains the observation time, and the second column contains the corresponding measurement. Deviations from this format must be indicated by providing the numbers of the columns, itime and idata, holding time and data information, respectively. If a third integer istd_dev is provided, an additional column is expected holding the standard deviation of the corresponding measurement. This allows one to specify individual standard deviations for each data point (the subcommand >>>> DEVIATION assigns a single standard deviation to all data points of a data set). The columns following command $>>>>$ DATA are read in free format. If a column contains non-numeric characters, command $>>>>$ FORMAT must be used.

\section{Example}

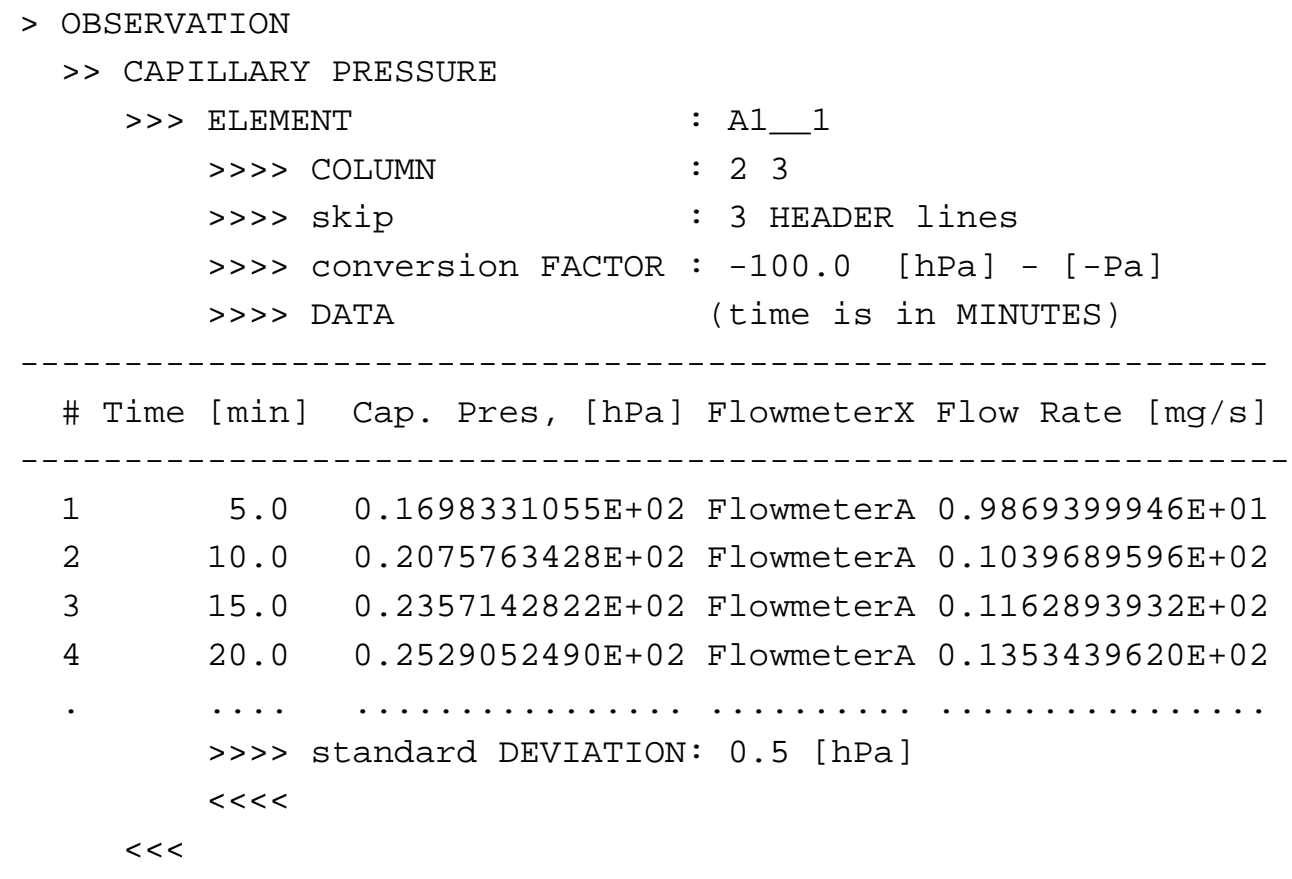




\section{Command}

>>> COMPONENT COmp_name/: icomp

Parent Command

$>>$ ELEMENT

$>>$ SOURCE (O)

\section{Subcommand}

$-$

\section{Description}

This command identifies a component either by its name (comp_name) or the component number (icomp). A list of allowable component names for the given EOS module can be obtained from the header of the iTOUGH2 output file.

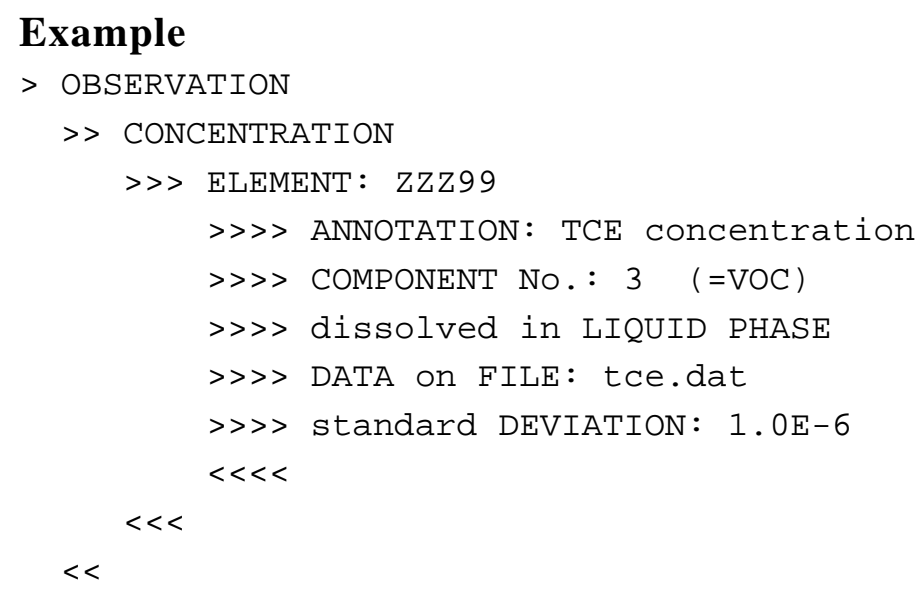

See Also

$>>>$ PHASE 


\section{Command}

>> COMPRESSIBILITY

\section{Parent Command}

$>$ PARAMETER

\section{Subcommand}

>> MATERIAL

\section{Description}

This command selects as a parameter the pore space compressibility $c_{\phi}$ (TOUGH2 variable $C O M(N M A T)$ ) of a certain rock type. Under fully liquid-saturated conditions, pore space compressibility estimates $c_{\phi}$ can be converted to a specific storage coefficient $S_{S}\left[\mathrm{~m}^{-1}\right]$ as follows:

$$
S_{s}=\phi \cdot \rho \cdot g\left(c_{l}+c_{\phi}\right)
$$

where $\phi$ is porosity, $\rho$ is density of water, $g$ is gravitational acceleration, and $c_{l} \approx 4 \cdot 4 \cdot 10^{-10}\left[\mathrm{~Pa}^{-1}\right]$ is water compressibility. Similarly, estimation of $c_{\phi}$ for grid blocks representing a well or borehole is useful for the determination of a dimensionless wellbore storage coefficient $C_{b h}$ :

$$
C_{b h}=\phi_{b h} \cdot \rho \cdot g \cdot V_{b h}\left(S_{l} \cdot c_{l}+S_{g} \cdot c_{g}+c_{\phi}\right)
$$

where $\phi_{b h}$ and $V_{b h}$ are the porosity and volume of the grid block representing the well, $S_{l}$ and $S_{g}$ are the liquid and gas saturation, and $c_{g}$ is gas compressibility which is approximately $1 / p$.

\section{Example}

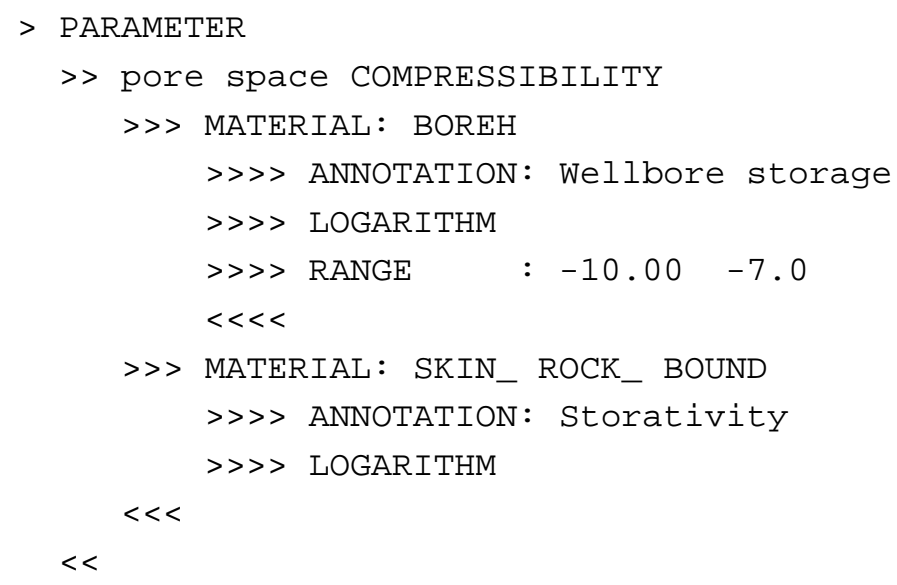

\section{See Also}




\title{
Command
}

$>$ COMPUTATION

\section{Parent Command}

$-$

\author{
Subcommand \\ $>>$ CONVERGE \\ $>$ ERROR \\ $>>$ JACOBIAN \\ $>>$ OPTION \\ $>>$ OUTPUT
}

\section{Description}

This is the first-level command for specifying a number of computational parameters, convergence criteria, program options, and output formats. The general format is as follows:

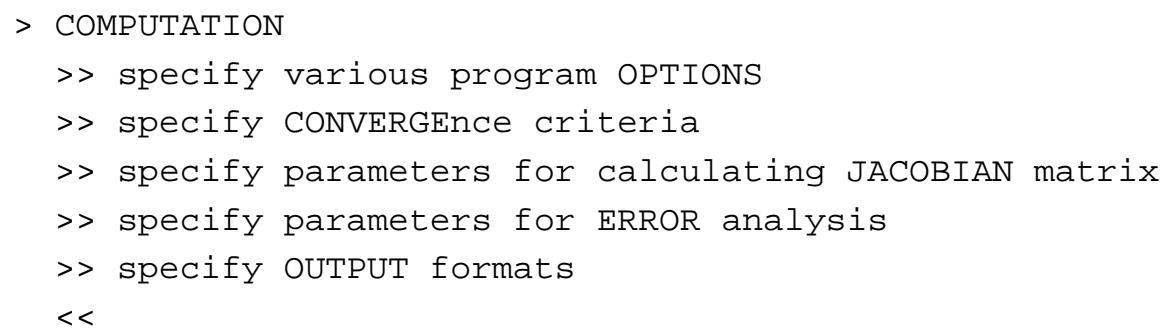

\section{Example}

$>$ COMPUTATION

>> CONVERGENCE criteria

$>>$ perform : 5 ITERATIONS

$<<<$

$>>$ JACOBIAN

>> use CENTERED finite difference quotient with

$>>>$ a relative parameter PERTURBation of : $0.5 \%$

$<<<$

$>>$ program OPTIONS

>> use LEAST-SQUARES objective function (default)

$>>$ allow the simulation to reach STEADY state

$<<<$

$>>$ OUTPUT

>>> generate PLOTFILE for: TECPLOT visualization software

$>>$ print all times in HOURS

$<<$

See Also

$-$ 


\section{Command}

>> CONCENTRATION (COMp_name/COMPONENT: icomp)

(phase_name/PHASE: iphase)

Parent Command

$>$ OBSERVATION

\section{Subcommand}

$>>$ ELEMENT

\section{Description}

This command selects as an observation type the concentration $\left[\mathrm{kg} / \mathrm{m}^{3}\right]$ of a component in a given phase. Concentration is defined as the product of mass fraction of component icomp in phase iphase times density of phase iphase. This observation type refers to an element. Component number icomp or component name comp_name, and phase number iphase or phase name phase_name depend on the EOS module being used. They are listed in the header of the iTOUGH2 output file, and can be specified either on the command line or using the two subcommands >>> COMPONENT and >>>> PHASE, respectively.

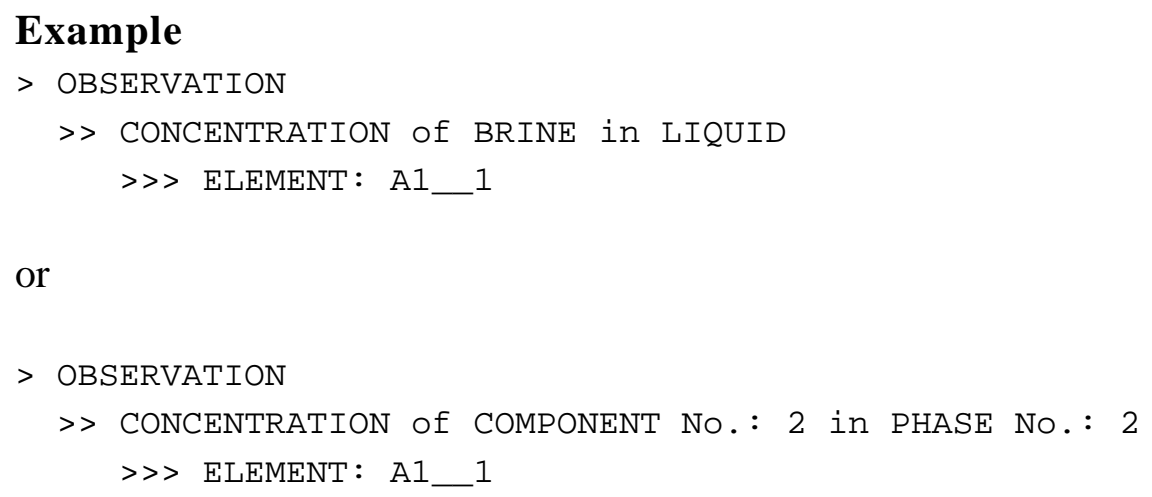

or

$>$ OBSERVATION

>> CONCENTRATION

>> ELEMENT: A1_1

>>> COMPONENT: 2

>>> LIQUID PHASE

See Also

$>$ MASS FRACTION 


\section{Command}

>> CONDUCTIVITY (WET/DRY)

\section{Parent Command}

$>$ PARAMETER

\section{Subcommand}

>> MATERIAL

\section{Description}

This command selects as a parameter the formation heat conductivity under fully liquidsaturated (keyword WET, default, TOUGH2 variable CWET (NMAT)) or desaturated conditions (keyword DRY, TOUGH2 variable CDRY(NMAT)).

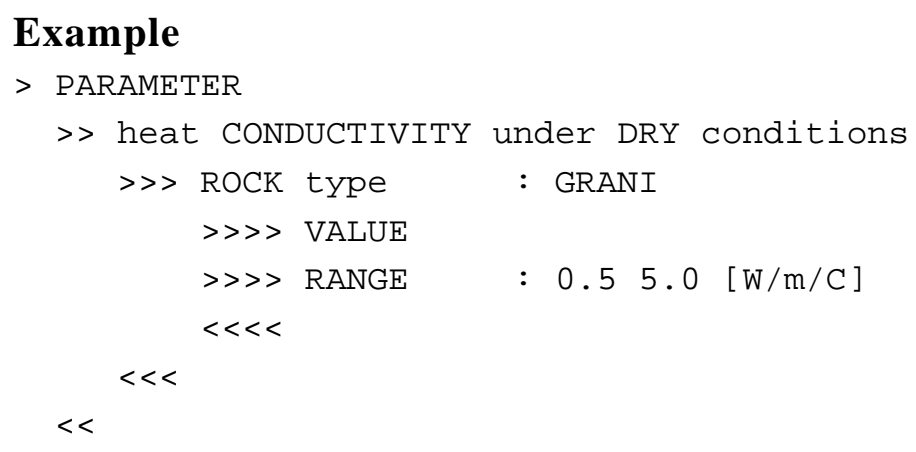

See Also 


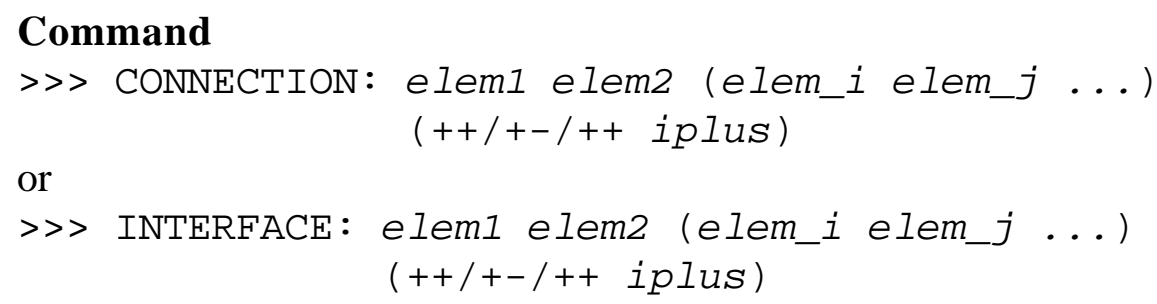

\section{Parent Command}

$>$ FLOW

\section{Subcommand}

all fourth-level commands in block > OBSERVATION

\section{Description}

This command reads pairs of element names defining a connection. Element names are designated by a three-character/two-integer (FORTRAN format: AAAII) code name. Blanks in the element names as printed in the TOUGH2 output file must be replaced by underscores (e.g., an element name specified in the TOUGH2 input file as 'B 007 ' is printed as 'B 0 7' to the TOUGH2 output file. Therefore, it has to be addressed in the iTOUGH2 input file as 'B_0_7').

Multiple connections can be specified, and iTOUGH2 calculates the sum or mean of all flow rates (see subcommands $>>>>$ SUM and >>> AVERAGE, respectively).

A sequence of iplus connections can be generated where the number of the first and/or the second element is increased by 1 . If only the first (second) element in a sequence of connections should be increased, use +- (-+). If both elements are to be increased, use + (or $++)$. The following two command lines are thus identical:

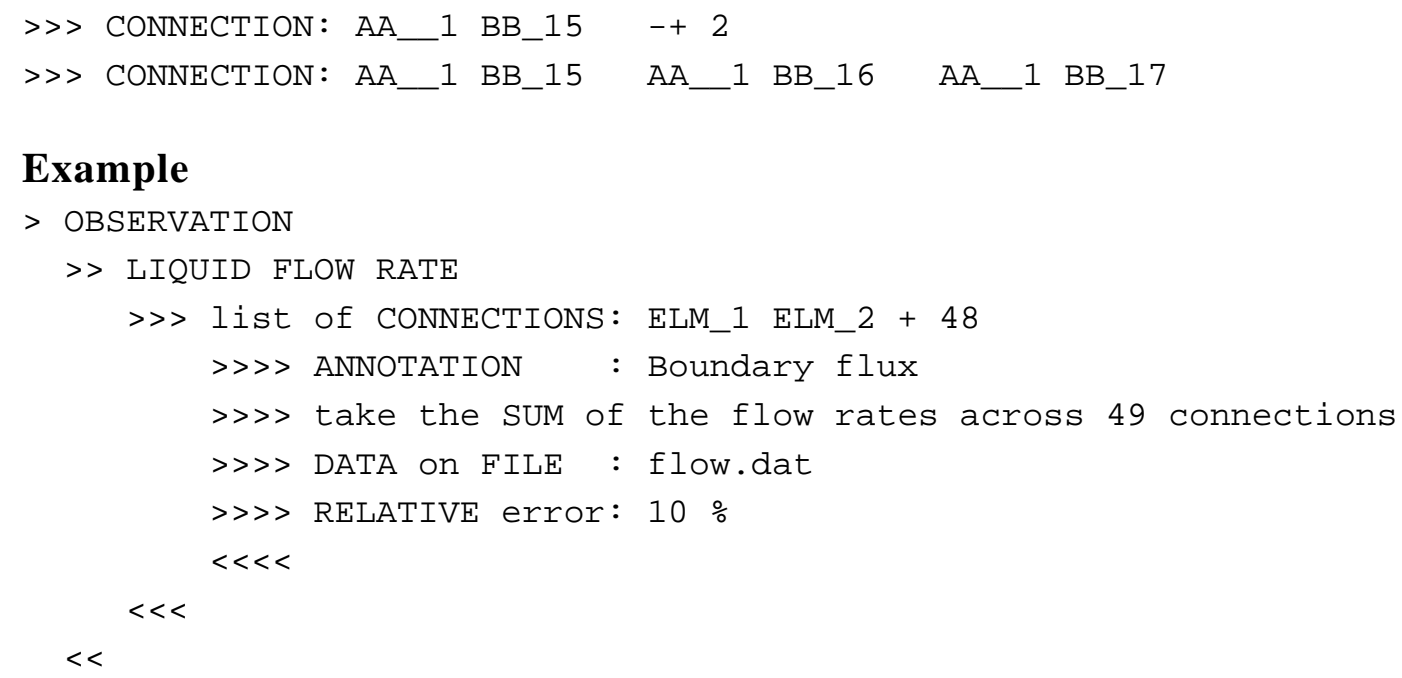

\section{Example}

$>$ OBSERVATION

$>>$ LIQUID FLOW RATE

$>>$ list of CONNECTIONS: ELM_1 ELM_2 + 48

$>>>$ ANNOTATION : Boundary flux

$>>>>$ take the SUM of the flow rates across 49 connections

$>>>$ DATA on FILE : flow.dat

$>>>$ RELATIVE error: $10 \%$

See Also

$>>>$ AVERAGE, $>>>$ SUM 


\section{Command}

>> CONSECUTIVE: max_iter1

\section{Parent Command \\ >> CONVERGE}

\section{Subcommand}

\section{Description}

By default, TOUGH2 simulations are stopped if 10 consecutive time steps converge with a single Newton-Raphson iteration because no update of primary variables occurs. This command allows changing the maximum number of allowable time steps with ITER=1 to max_iterl.

Consecutive time steps with no update of primary variable occur if:

(1) steady-state is reached;

(2) calibration or printout times are too narrowly spaced;

(3) the maximum time step size (TOUGH2 variable DELTMX) is too small;

(4) the initial time step (TOUGH2 variable DELTEN or DLT(1)) is too small;

(5) a small time step is taken to land on a calibration or printout time.

Only (1) is an acceptable TOUGH2 convergence (see command >> STEADY-STATE). All the other reasons may lead to premature termination of a TOUGH2 simulation. Convergence problems are more often encountered in iTOUGH2 than in a standard TOUGH2 simulation because many parameter combinations are submitted. This command makes TOUGH2 more tolerant of this kind of convergence failure. It is important, however, that max_iterl is only increased to overcome temporary convergence problems during the optimization, i.e., the final parameter set should yield a TOUGH2 simulation without convergence problems. Calibration points should not be spaced too narrowly in time (see command >> TIME). Command >> ADJUST can be used to overcome problem (4). Note that a special time stepping procedure is incorporated in iTOUGH2 to avoid problem (5).

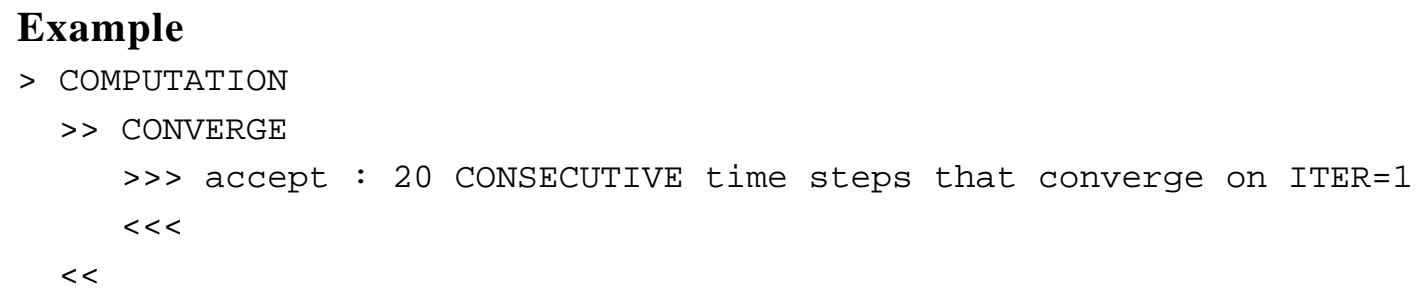

See Also

> TIME, $>>$ ADJUST, $>>$ REDUCTION 


\section{Command}

>> CONTENT (phase_name/PHASE: iphase)

\section{Parent Command}

$>$ OBSERVATION

\section{Subcommand}

$>>$ ELEMENT

\section{Description}

This command selects as a parameter the content of phase iphase as an observation type. Phase content is defined as the product of saturation and porosity.

The phase name phase_name or phase number iphase, which depend on the EOS module being used, are listed in the iTOUGH 2 header, and can be specified either on the command line or using subcommand $>>>>$ PHASE.

Estimating phase content and phase saturation is identical only if porosity remains constant. Porosity can be variable (i) due to compression of the pore space (i.e., if TOUGH2 variable COM (NMAT) is not zero), and (ii) if porosity is one of the parameters to be estimated and is updated during the inversion.

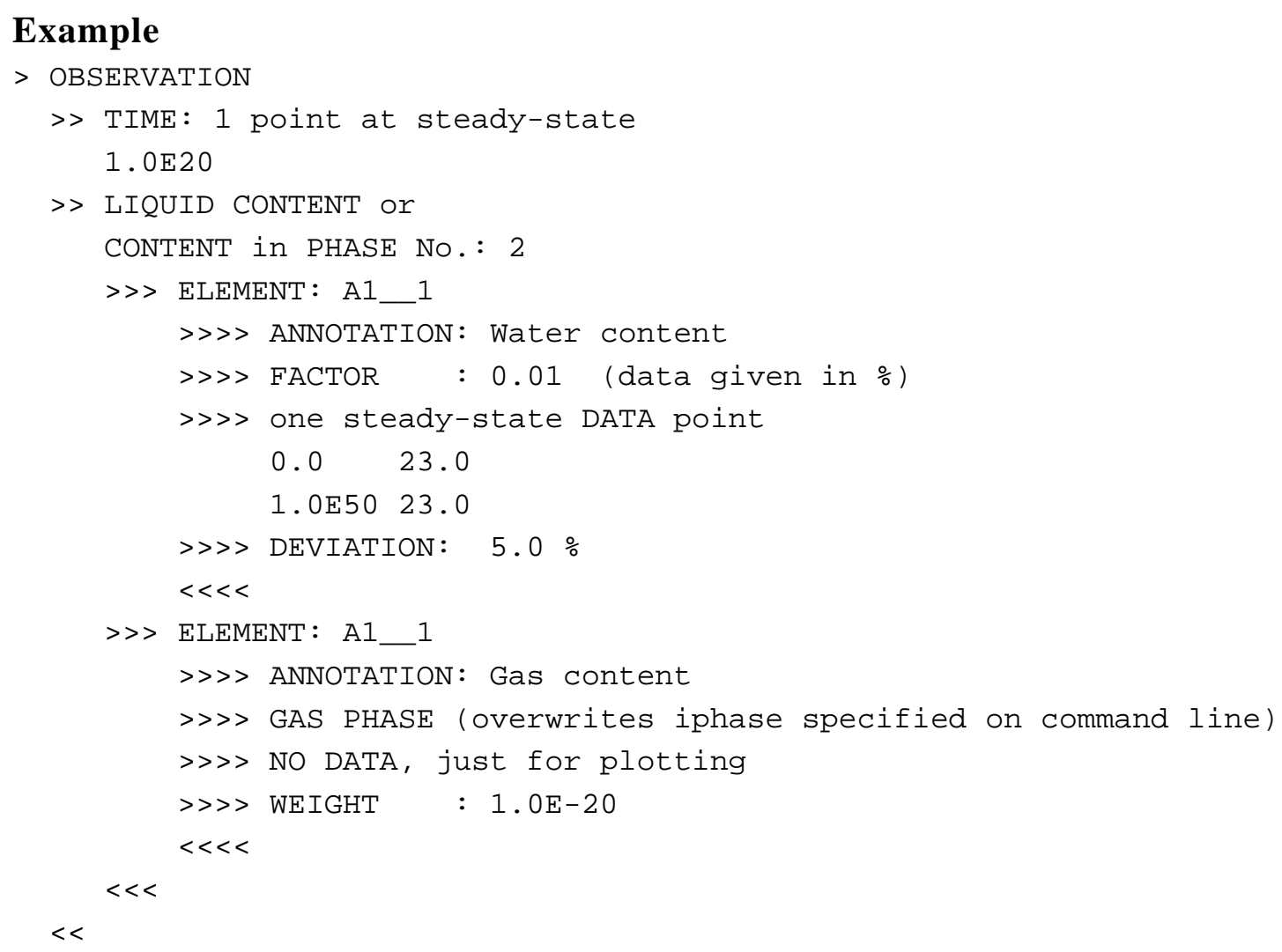

See Also 


\section{Command}

>> CONVERGE

or

$>$ STOP

or

>> TOLERANCE

Parent Command

$>$ COMPUTATION

\section{Subcommand}

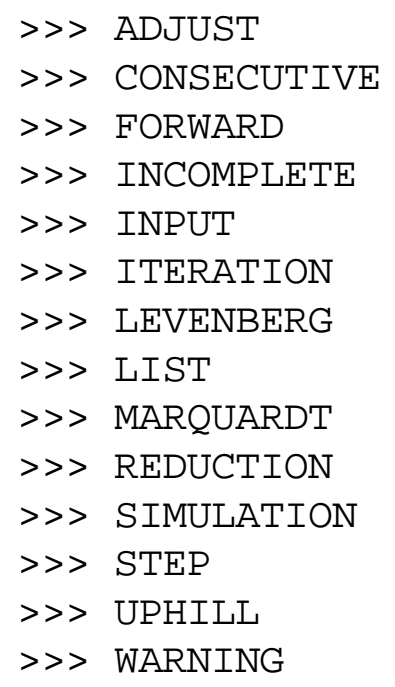

\section{Description}

This is the parent command of a number of subcommands that deal with tolerance measures and convergence criteria for the inversion and, to a certain extent, the TOUGH2 simulation.

\section{Example}

$>$ COMPUTATION

>> CONVERGEnce criteria

>> ignore WARNING messages, then

$>>$ perform : 5 ITERATIONS

>> stop if more than : 5 unsuccessful UPHILL steps are proposed

>> allow for : 20 CONSECUTIVE time steps converging on ITER=1

$>>$ and : 20 time step REDUCTIONS

>> accept : 6 INCOMPLETE TOUGH2 runs

>> the initial LEVENBERG parameter shall be : 0.01

$>>>$ use the default value $(=:$ 10.0) for the MARQUARDT parameter $<<<$

See Also

$>$ OPTION 


\section{Command}

>>> CORRELATION: (-) rCOrY

\section{Parent Command}

$>>$ SELECT

\section{Subcommand}

$-$

\section{Description}

This command defines one of the criteria used for automatic parameter selection. It examines the ratio between the apparent conditional standard deviation $\sigma_{p} *$ and the joint standard deviation $\sigma_{p}$ as a measure of overall parameter correlation (since the calculation is performed far from the minimum, the standard deviations cannot be interpreted as actual estimation uncertainties):

$$
\chi=\frac{\sigma_{p} *}{\sigma_{p}} \quad 0<\chi \leq 1
$$

Those parameters with a ratio larger than $\mid$ rcorr $\mid$, i.e., the most independent parameters, are selected. Strongly correlated parameters are (temporarily) excluded from the optimization process.

If a negative value is given for $r c o r r$, the selection criterion is relaxed with each iteration $k$, and reaches zero for the last iteration max_iter, i.e., all parameters are selected for the final step.

$$
\operatorname{rcorr}_{k}=|r c o r r| \cdot\left(1-\frac{k}{\text { max_iter }_{-}}\right)
$$

The choice for $r$ corr depends on the number of parameters $n$ specified in block $>$ PARAMETER. The more parameters are estimated simultaneously, the higher are parameter interdependencies. This fact should be acknowledged by specifying a smaller value for $\mid$ rcorr $\mid$ if $n$ increases.

\section{Example}

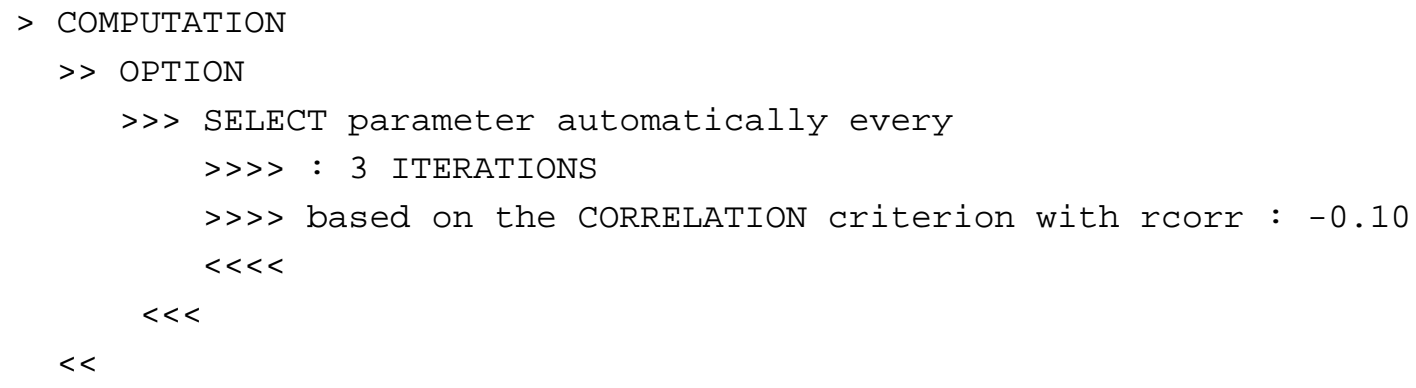

See Also

>>> ITERATION (s), >>>> SENSITIVITY 


\section{Command}

>> COVARIANCE (FILE: file_name)

\section{Parent Command}

$>$ OBSERVATION

\section{Subcommand}

\section{Description}

This command reads the diagonal elements of the a priori covariance matrix $\mathbf{C}_{z z}$. Usually the variances of the observations are specified separately for each data set using command $>>>>$ DEVIATION (O), or they are provided as a separate column along with the data (see commands $>>>>$ COLUMN and $>>>>$ DATA). As an alternative, one can assign or overwrite variances using the second-level command $>>$ COVARIANCE, followed by two columns holding index $i$ and variance $C_{i i}$. Since this command addresses elements of the assembled covariance matrix, the user must provide the index that corresponds to the position of the observation in vector $\mathbf{z}$. This information is best retrieved from the iTOUGH2 output file after running one forward simulation. Note that the index changes whenever the number of observations, parameters, or calibration times is changed. The variances can also be read from a covariance file which has to contain three columns, holding index $i$, index $j$, and the (co-)variance $C_{i j}$ (this is the same format as the one on the covariance file generated by command $>>>$ COVARIANCE). Despite the fact that two indexes must be provided, only diagonal elements, i.e., $i=j$, will be accepted as input.

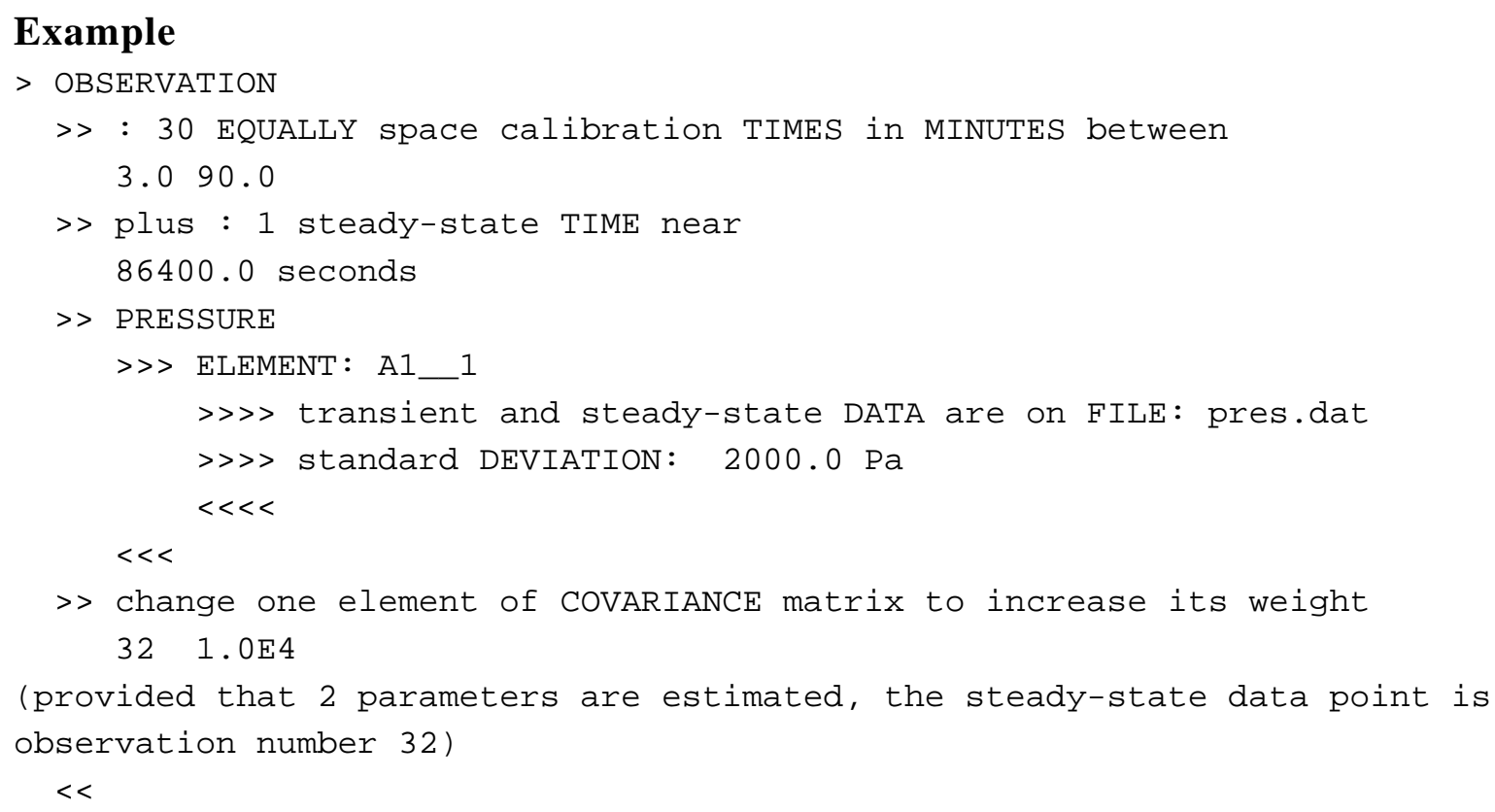

See Also

$>>$ COVARIANCE, $>>>>$ COLUMN, $>>>$ DEVIATION, $>>>$ DATA 


\section{Command}

$>>$ COVARIANCE

\section{Parent Command}

$>$ OUTPUT

Subcommand

\section{Description}

This command generates a file with extension ".cov " with the covariance matrix of the calculated system response:

$$
\mathbf{C}_{\hat{z} \hat{z}}=\mathbf{J} \mathbf{C}_{p p} \mathbf{J}^{T}=s_{0}^{2} \cdot \mathbf{J}\left(\mathbf{J}^{T} \mathbf{C}_{z z}^{-1} \mathbf{J}\right)^{-1} \mathbf{J}^{T}
$$

Note that $\mathbf{C}_{\hat{z} \hat{z}}$ is a square matrix of dimension $m \times m$.

\section{Example}

$>$ COMPUTATION

$>>$ OUTPUT

$>>>$ print COVARIANCE matrix of calculated system response $<<<$

$<<$

See Also 


\title{
Command
}

\author{
>> CUMULATIVE (comp_name/COMPONENT: icomp) \\ (phase_name/PHASE: iphase)
}

Parent Command

$>$ OBSERVATION

\section{Subcommand}

>> SOURCE

\section{Description}

This command selects as an observation type the cumulative injection or production of component icomp or phase iphase. This observation type refers to a sink or source code name. It can be used when time-dependent generation rates are to be estimated where the total amount of injected or produced fluid is approximately known, or if the total generation rate is prescribed in block GENER, but the phase composition of the produced fluid is variable and sensitive to the parameters of interest. Finally, the cumulative amount of injected or produced fluid can be used as an observable variable for wells on deliverability. Note that the cumulative mass of a phase produced in an element strongly depends on the composition of the produced fluid mixture according to the options provided by TOUGH2 flag $M O P(9)$.

Component number icomp or component name comp_name, and phase number iphase or phase name phase_name depend on the EOS module being used. They are listed in the iTOUGH2 header and can be specified either on the command line or using the two subcommands >>>> COMPONENT and >>>> PHASE, respectively. If neither a phase nor component number is specified, the total, cumulative mass of all phases or components will be calculated.

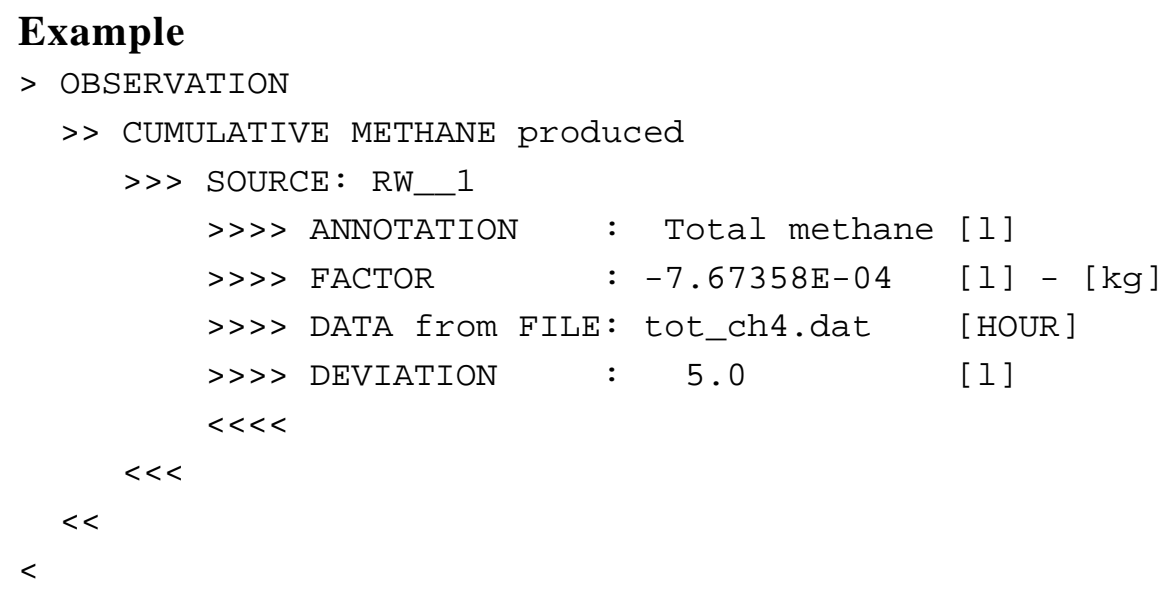

See Also 


\section{Command}

>>> DATA (time_unit) (FILE: file_name)

Parent Command

all third-level commands in block > OBSERVATION

\section{Subcommand}

\section{Description}

This command reads a list of observation times and the corresponding data. Each list will be referred to as a data set in the iTOUGH2 output file. An annotation is generated for each data set, or a string can be supplied by the user for its identification (see command >>> ANNOTATION).

Data can be supplied either directly following the command line or on a separate file (use keyword FILE followed by the name of the data file after a colon).

Time and data must be arranged in columns. They are read in free format. Data are accepted until a FORTRAN input error occurs. The total number of data points read by iTOUGH2 is printed to the iTOUGH2 output file and should be checked for consistency.

By default, the first column is expected to hold the observation times, and the second column the data values. Deviations from this format are possible (see commands $>>>>$ COLUMN, >>>> FORMAT, >>> SET (०), and >>>> HEADER for details). Data can also be represented by a polynomial or a user-specified function (see commands $>>>>$ POLYNOM and >>>> USER, respectively).

Observation times do not need to coincide with the calibration times defined by command >> TIME (O). Linear interpolation is performed for calibration times that fall between observation times. This requires, however, that the first observation time is earlier than the first calibration time, and that the last observation time is later than the last calibration time. If this condition is not met, command >>>> WINDOW should be used. Only one time window can be specified for each data set, i.e., multiple data sets must be provided if multiple time windows are needed.

If time is not given in seconds, the appropriate time unit (MINUTE, HOUR, DAY, WEEK, MONTH, YEAR) must be specified on the command line. If the units of the data points are different from the standard units used in TOUGH2, a conversion factor must be provided through command $>>>>$ FACTOR.

If no observed data are available (e.g., when performing design calculations prior to testing, or when using iTOUGH2 for generating time series plots), a dummy data set must be supplied. Alternatively, command >>>> NO DATA can be used. 


\section{Example}

$>$ OBSERVATION

$>>$ GAS PRESSURE

>> in ELEMENT: A1_1

>>> DATA follow in default format, time (sec) vs. pressure (Pa)

1.0100000 .0

$10.0 \quad 101343.8$

$20.0 \quad 105991.3$

$30.0 \quad 108965.9$

$60.0 \quad 115003.8$

............

$3600.0 \quad 218762.0$

$>>>>$ standard DEVIATION: $5000.0 \mathrm{~Pa}$

$<<<<$

$<<<$

$>>$ LIQUID FLOW rate

>> CONNECTION B1_1 B1_2

>>> HEADER contains : 3 lines to be skipped

>>> time and value are in COLUMNS: 36

>>> conversion FACTOR is : $-1.6667 \mathrm{E}-5$ [ml/min] to [ $-\mathrm{kg} / \mathrm{sec}]$

$>>>$ use SET No. : 3

>>> DATA are provided on FILE : flow.dat with time in MINUTES

$>>>>$ a RELATIVE measurement error of : $5.0 \%$ is assumed

$<<<<$

$<<<$

$>>$ BRINE CONCENTRATION in LIQUID PHASE

>> ELEMENT: $\mathrm{C} 1$ _ 1

>>> NO DATA available, just for plotting

$>>>$ assign small WEIGHT of : $1.0 \mathrm{E}-20$

$<<<<$

$<<<$

$<<$

\section{See Also}

>>> ANNOTATION, $>>>>$ COLUMN, $>>>>$ FACTOR, $>>>>$ FORMAT,

$>>>$ HEADER, $>>>$ PICK, $>>>>$ POLYNOM, >>> SET (0),

$>>>$ USER, $>>>>$ WINDOW 


\section{Command}

$>>$ DEFAULT

or

$>>$ MATERIAL: DEFAU

\section{Parent Command}

$>$ CAPILLARY

$>>$ INITIAL

$>>$ RELATIVE

\section{Subcommand}

all fourth-level commands in block > PARAMETER

\section{Description}

Parameters of the default relative permeability and capillary pressure functions (TOUGH2 block RPCAP) or default initial conditions (TOUGH2 block PARAM.4) are addressed by command $>>>>$ DEFAULT. Alternatively, a material name DEFAU can be provided following command $>>>>$ MATERIAL.

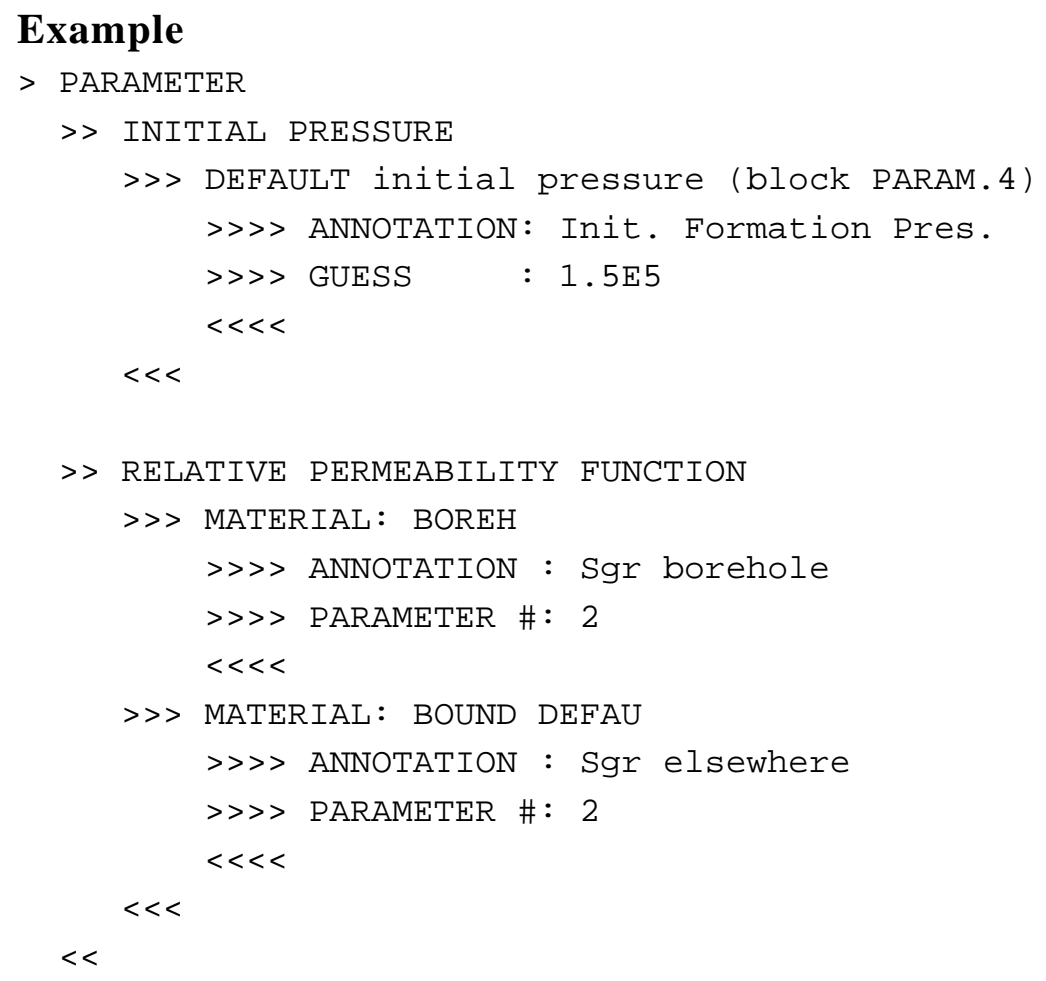

See Also

>> MATERIAL, >> MODEL 


\section{Command}

>> DELTFACT: deltfact

\section{Parent Command}

>> CONVERGE

\section{Subcommand}

$-$

\section{Description}

In TOUGH2, time step size can be controlled in various ways (see Pruess [1987] for a description of input variables DELTEN, DLT, DELTMX, NOITE, REDLT, and $M O P(16)$ ). The time step is also automatically adjusted in order for the simulation time to land on any of the specified calibration or printout times. This may lead to very small time steps or even convergence failures (see command $>>>$ CONSECUTIVE).

In iTOUGH2, the proposed time step is increased up to a factor of delt fact (default: 1.1) if this increase allows the simulation to reach the next calibration or printout time. This may lead to time stepping different from standard TOUGH2.

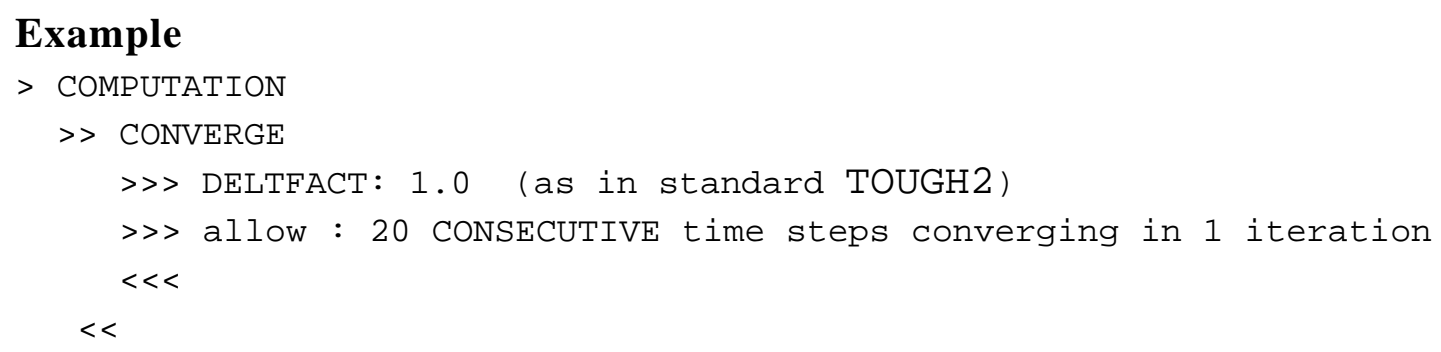

See Also

>> CONSECUTIVE 


\section{Command}

>> DESIGN

\section{Parent Command}

>> OPTION

Subcommand

Description

(synonym for command $>>$ SENSITIVITY)

Example

(see command >> SENSITIVITY)

See Also

>> SENSITIVITY 


\section{Command}

>>> DEVIATION: sigma

\section{Parent Command}

all third-level commands in block > PARAMETER

\section{Subcommand}

\section{Description}

This command specifies the standard deviation $\sigma_{p_{i}}$ of the initial parameter guess. Prior information about model parameter $i$ will be weighted by $1 / \sigma_{p_{i}}$, i.e., the difference between the prior information value $p_{i}{ }^{*}$ and the estimate $\hat{p}_{i}$ contributes to the objective function as follows:

$$
\text { weighted squared residual from prior information }=\frac{\left(p_{i}^{*}-\hat{p}_{i}\right)^{2}}{\sigma_{p_{i}}^{2}}
$$

Commands for specifying the standard deviation are:

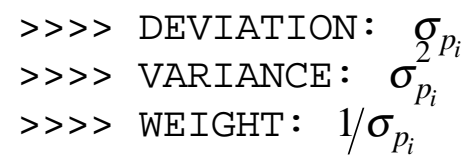

By default, prior information is not weighted, i.e. $\sigma_{p_{i}}^{2}=\infty$.

The standard deviation reflects the uncertainty associated with the initial guess. If the initial guess is to be weighted, prior information should originate from an independent source. For example, if porosity will be estimated based on transient pressure data, the prior information value should be taken from a "direct" porosity measurement, e.g. using mercury-porosimetry or oven-drying methods. In these cases, the measured parameter values $p_{i} *$ are considered to be additional data points which serve as a physical plausibility criterion for the estimate $\hat{p}_{i}$. The $p_{i}{ }^{*}$ values, along with the observations of the system state $z_{i}^{*}$, are then weighted according to their uncertainties (see $>>>>$ DEVIATION (O)). Note that the relative weighting between prior information and the observations $z_{i}^{*}$ depends on the number of calibration points selected. If many transient data points are available, a smaller standard deviation $\sigma_{p_{i}}$ may be specified to increase the relative weight of prior information. In many cases, appropriately weighting the initial guess makes an ill-posed inverse problem unique. Furthermore, the solution becomes more stable if a parameter is not very sensitive. However, using $1 / \sigma_{p_{i}}$ as a regularization parameter to improve the ability to obtain a unique solution with a poorly conceptualized inverse problem is not recommended. Erratic behavior of a parameter during the inversion should be taken as an indication that the data do not contain sufficient information for the determination of the parameter. Differences between parameter values that are independently determined from laboratory experiments and inverse modeling suggest the presence of a systematic error or scaling problem. These inconsistencies should be resolved rather than averaged out. 
The standard deviation $\sigma_{p_{i}}$ is also used to scale the columns of the Jacobian matrix. While the solution of the inverse problem is not affected by the choice of the scaling factor, all the qualitative sensitivity measures are directly proportional to $\sigma_{p_{i}}$. If prior information is not weighted, the scaling factor is taken to be $10 \%$ of the respective parameter value. Command >>> VARIATION should be used to change the default scaling factor without concurrently assigning a weight to prior information.

When performing uncertainty propagation analyses, $\sigma_{p_{i}}$ designates the parameter uncertainty affecting the model prediction. It is used to generate a set of random parameter values for Monte Carlo simulations, and it represents the standard deviation of a normal distribution if performing linear uncertainty propagation analysis (for more details see commands >> MONTE CARLO and >> FOSM, respectively).

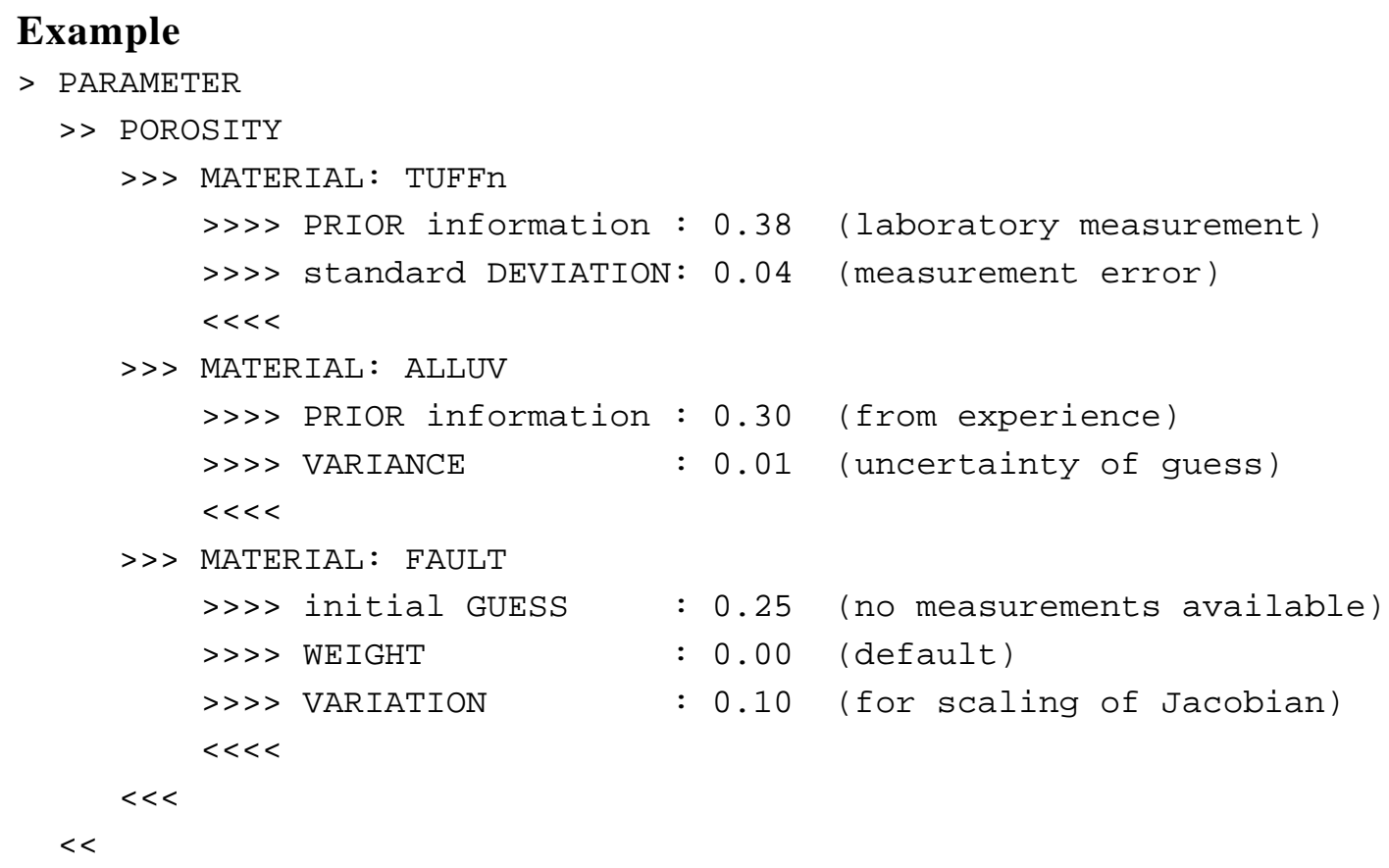




\section{Command}

>>> DEVIATION: sigma

\section{Parent Command}

all third-level commands in block > OBSERVATION

\section{Subcommand}

\section{Description}

This command specifies the standard deviation $\sigma_{z_{i}}$ of the observations. The squares of the standard deviations constitute the diagonal elements of the apriori covariance matrix.

The specified value sigma is assigned to all data points of the corresponding data set. It must be given in the same units as the data (ses command $>>>>$ FACTOR). Individual values for each calibration point can be explicitly specified using command $>>>>$ COLUMN or $>>$ COVARIANCE, or are calculated as a fraction of the measured value if using command $>>>$ RELATIVE.

The standard deviation should represent the expected variability of the final residuals. In the absence of modeling errors, the standard deviation is equivalent to the measurement error. A reasonable value can be derived by visual examination of the data, i.e., by estimating the standard deviation of the differences between the observed values and a line representing the expected match; note that this procedure is based on the assumption that time averages can be used to calculate the ensemble average, i.e., that the data set is a result of an ergodic process. The inverse of the a priori covariance matrix is used to weight the fitting error. It also scales observations of different types and units. In the framework of maximum likelihood theory, the covariance matrix constitutes the stochastic model along with the assumption of normality and independence.

The parameter estimates are not affected by the absolute values of $\sigma_{z_{i}}$, but only by the ratios $\sigma_{z_{i}} / \sigma_{z_{j}}$. It is suggested, however, to use reasonable values that are related to the measurement error. If the final residuals are - on average - significantly larger than the $a$ priori specified standard deviations, the Fisher model test fails. The a posteriori standard deviations of the final residuals are printed in the output for each data set for comparison purposes.

Alternative commands are:

>>>> DEVIATION $: \sigma_{z_{i}}$
>>>> VARIANCE $: \sigma_{z_{i}}$
>>>> WEIGHT $: 1 / \sigma_{z_{i}}$

The following command lines are thus equivalent:

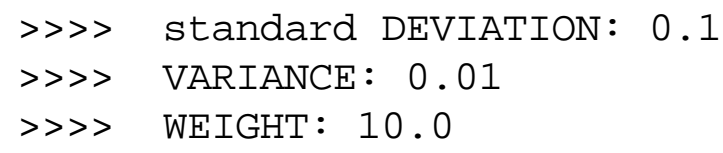




\section{Example}

$>$ OBSERVATION

$>>$ PRESSURE

>> ELEMENT : AA412

>>> conversion FACTOR: 1E5 from [bar] to [Pa]

>>> DATA on FILE: pressure.dat

$>>>$ standard DEVIATION: 0.05 [bar]

$<<<<$

$<<<$

$<<$

\section{See Also}

> COVARIANCE, $>>>>$ AUTO, $>>>>$ COLUMN, $>>>>\operatorname{DEVIATION~(p),~}$ $>>>>$ RELATIVE, $>>>>$ VARIANCE, $>>>>$ WEIGHT 


\section{Command}

>> DIRECT

\section{Parent Command}

$>$ OPTION

or

>> CONVERGE

\section{Subcommand}

$-$

\section{Description}

(synonym for command $>>>$ FORWARD (o))

\section{Example}

(see command >> FORWARD (o))

See Also

>> FORWARD (O) 


\section{Command}

>> DRAWDOWN (phase_name/PHASE: iphase)

\section{Parent Command}

$>$ OBSERVATION

\section{Subcommand}

>> ELEMENT

\section{Description}

This command selects as an observation type the pressure drawdown during a pumping test. This observation type refers to one or more elements. The drawdown is calculated from a reference pressure, which is the pressure at the specified element at the time of the first active calibration point for that data set, i.e., a calibration time greater than the starting time of the simulation must be provided, indicating the beginning of the pumping period; the corresponding observation is a drawdown of zero. Note that the sensitivity of this calibration point is zero by definition, i.e., the first data point is not used for calibration. If drawdown is measured in meters, command $>>>>$ FACTOR must be used to convert the units to Pascals. The phase name phase_name or phase number iphase, which depend on the EOS module being used, are listed in the header of the iTOUGH2 output file. They can be specified either on the command line or using subcommand >>>> PHASE. If no phase is specified, iTOUGH 2 takes the pressure drawdown of the first phase which is usually the reference pressure.

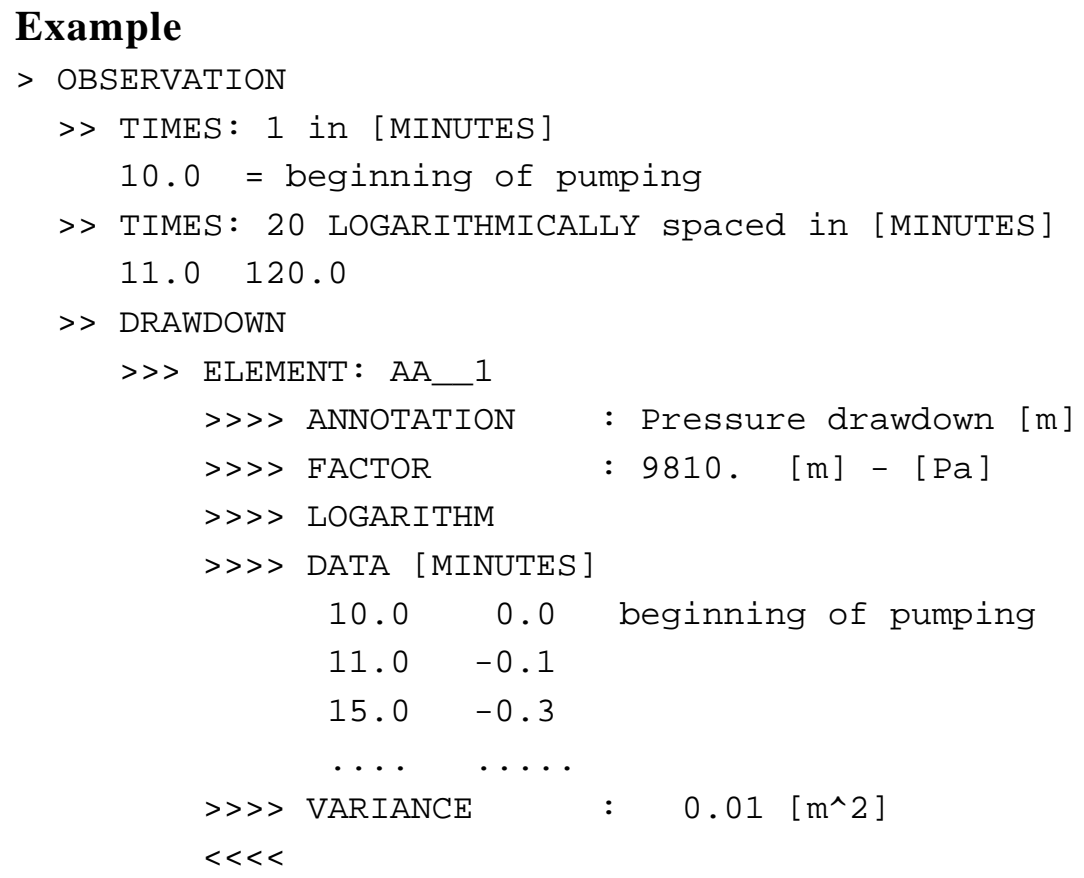




\section{Command}

$>$ DRIFT

\section{Parent Command}

$>$ PARAMETER

\section{Subcommand}

$>>$ NONE

$>>\mathrm{SET}$

\section{Description}

This command selects as a parameter the slope of a time-dependent trend. The trend is added to the TOUGH2 output referring to a specific data set:

$$
z=z_{\text {TOUGH } 2}+d \text { rift } \cdot \text { time }
$$

where $($ drift $\cdot$ time $)$ is the trend added to the calculated TOUGH2 output $z_{\text {TOUGH2 }}$. The result $z$ is compared to the measurement $z^{*}$ of the corresponding data set.

This option allows removal of a trend in the data (for example, a flowmeter may exhibit an unknown offset and time-dependent trend that needs to be estimated). A non-zero value must be provided as initial guess through the iTOUGH2 input file using command $>>>$ GUESS. The data set is identified by number using command $>>>\operatorname{SET}(\mathrm{p})$.

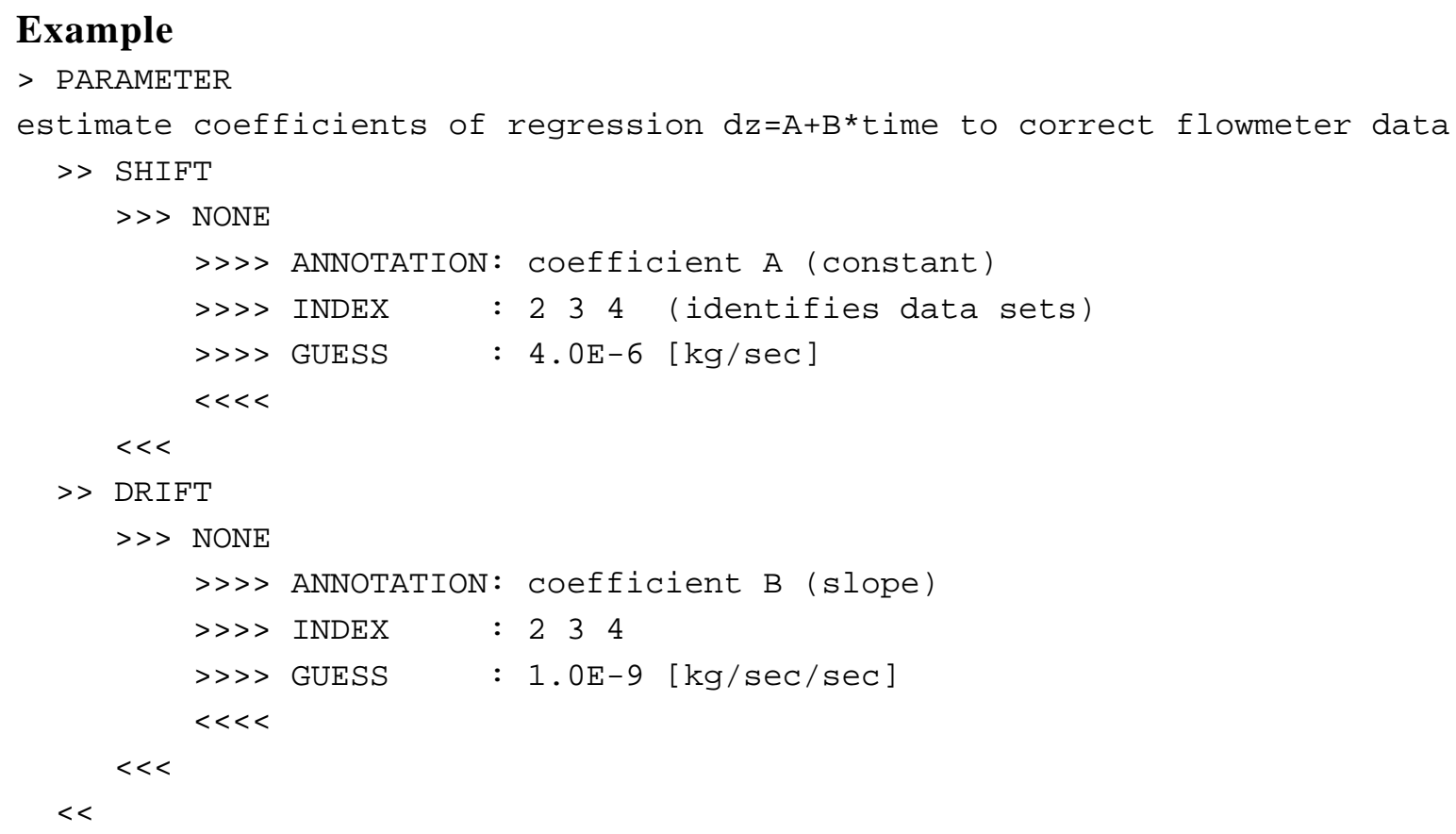

See Also

$>$ FACTOR, $>$ LAG, $>$ SHIFT, $>>>\operatorname{SET}(p),>>>\operatorname{INDEX}(\mathrm{p})$ 


\section{Command}

>> ELEMENT: eleme (eleme_i...) (+ iplus)

or

>> GRID BLOCK: eleme (eleme_i ...) (+ iplus)

\section{Parent Command}

all second-level commands in block > OBSERVATION requiring element names.

\section{Subcommand}

all fourth-level commands in block > OBSERVATION

\section{Description}

This command reads one or more element names. Most observation types refer to a variable that is associated with a grid block (as opposed to a connection or sink/source name). Element names are designated by a three-character/two-integer (FORTRAN format: AAAII) code name. Blanks in the element names as printed in the TOUGH2 output file must be replaced by underscores (e.g. an element name specified in the TOUGH2 input file as 'B 007 ' is printed as 'B 0 7' in the TOUGH2 output file. Therefore, it has to be addressed in the iTOUGH2 input file as 'B_0_7').

Multiple elements can be specified, and iTOUGH2 calculates the sum or mean of the corresponding output variable (see subcommands >>> SUM and >>>> MEAN, respectively).

A sequence of iplus elements can be generated by increasing the number of the last element. The following two command lines are identical:

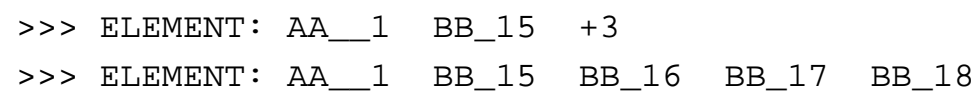

\section{Example}

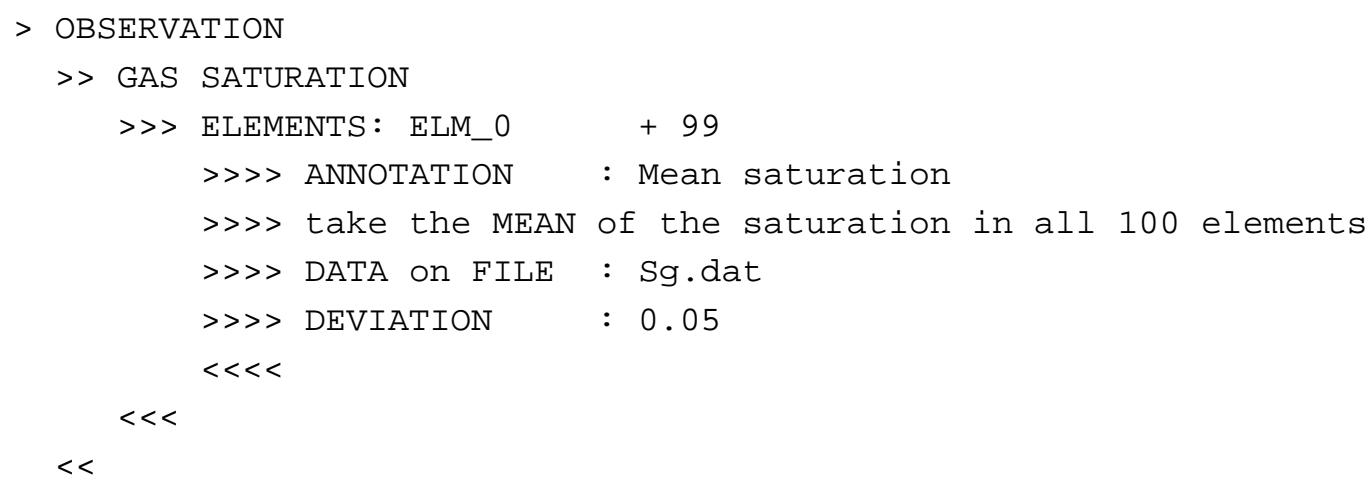

See Also

>> CONNECTION, >> SINK, >>> MEAN, >>>> SUM 


\section{Command}

>> EMPIRICAL (MATRIX: ndim) (ITOUGH2) (CORRELATION)

\section{Parent Command}

$>$ ERROR

\section{Subcommand}

$-$

\section{Description}

This command must be used in combination with command $>>$ MONTE CARLO to invoke stochastic simulations of correlated parameters using Empirical Orthogonal Functions (EOF). EOF is a variant of Monte Carlo simulations to quantify the uncertainty of model predictions as a result of parameter uncertainty. Many parameter sets are generated, following the predefined covariance matrix $\mathbf{C}_{p p}$. The $i$ th parameter set $\mathbf{Y}_{i}$ is obtained by linear combination of the eigenvectors $\mathbf{u}_{k}$ of $\mathbf{C}_{p p}$ and stochastic coefficients $\phi_{k}\left(\xi_{i}\right)$ :

$$
\begin{gathered}
\mathbf{Y}_{i}=\sum_{k=1}^{n} \mathbf{u}_{k} \phi_{k} \\
\phi_{k}\left(\xi_{i}\right)=\xi_{i} \cdot \sqrt{a_{k}}
\end{gathered}
$$

where $\xi$ is a standard normal distributed random variable, and $a_{k}$ is the $k$ th eigenvalue of $\mathbf{C}_{p p}$. For more details, see Kitter $\phi d$ and Gottschalk [1997].

The elements of matrix $\mathbf{C}_{p p}$ can be supplied using one of the following options:

(1) Provide indices and elements of $\mathbf{C}_{p p}$. Example:

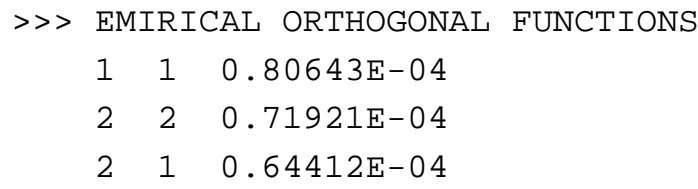

Use keyword CORRELATION if off-diagonal term is correlation coefficient instead of covariance. Example:

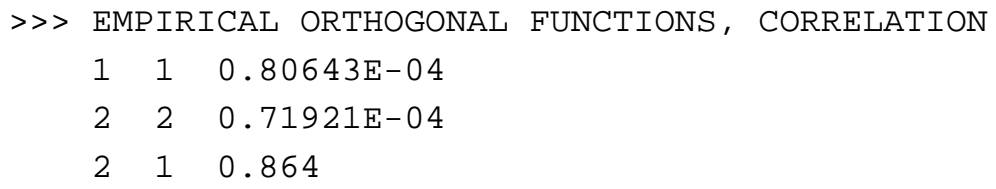


(2) Provide keyword MATRIX, followed by a colon and the dimension ndim of the square matrix $\mathbf{C}_{p p}$. The lower triangle of the covariance matrix is then provided on exactly ndim additional lines. If keyword CORRELATION is present, the offdiagonal terms represent correlation coefficients rather than covariances. Example:

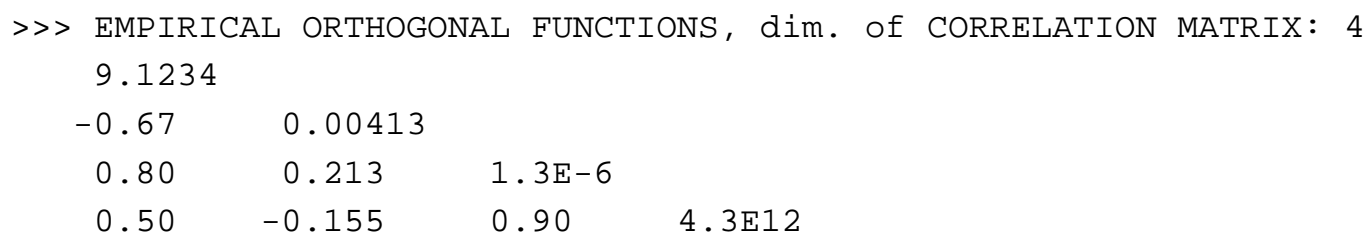

(3) If calculated during a previous iTOUGH2 inversion, the covariance matrix can be taken from the iTOUGH2 output file and directly copied after the command line. This option is invoked by keyword iTOUGH2. The matrix will be read by formatted input, so it is crucial that the correct format is maintained. If ndim is greater than 6 , the matrix is split in multiple submatrices. All submatrices must be copied exactly as they were printed to the iTOUGH2 output file. Example:

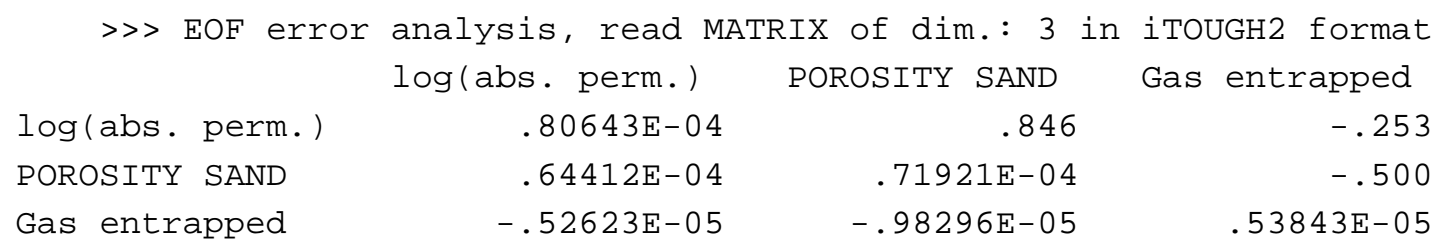

\section{Example}

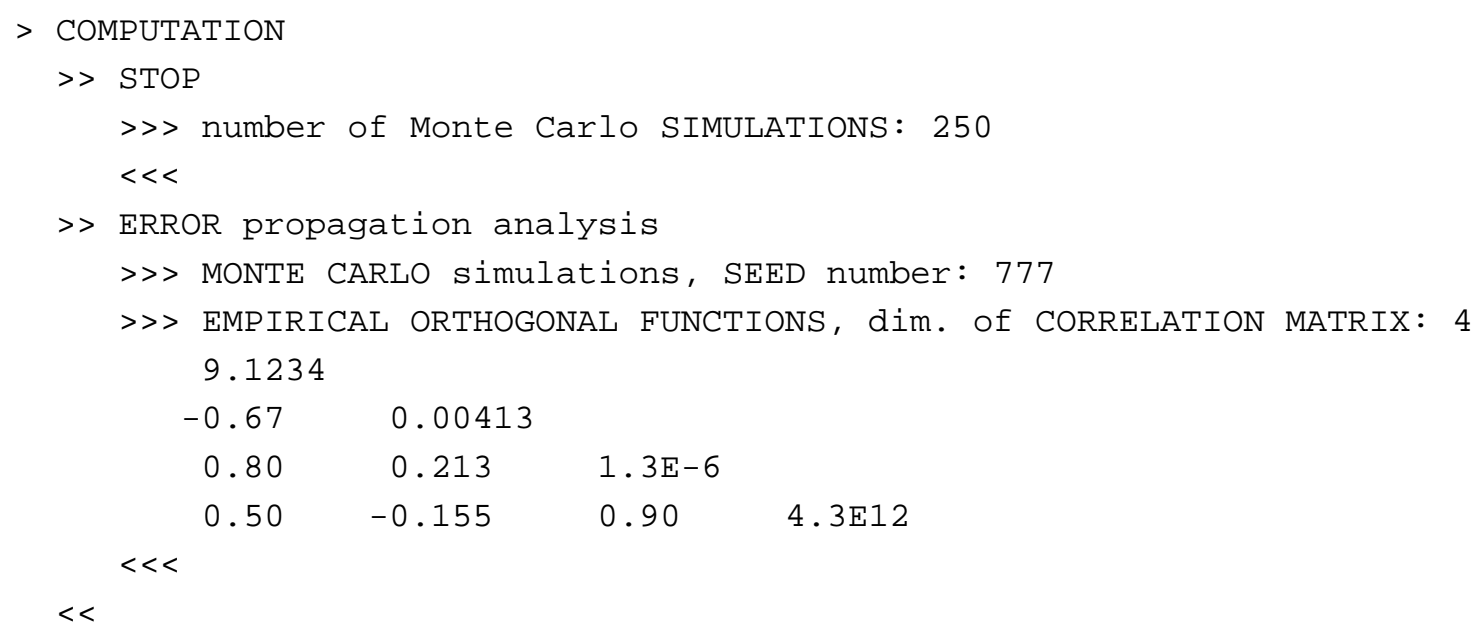

See Also

>> FOSM, >> MONTE CARLO 


\section{Command}

$>$ ENTHALPY

\section{Parent Command}

$>$ PARAMETER

\section{Subcommand}

$>>$ SOURCE

\section{Description}

This command selects as a parameter the fixed specific enthalpy of the injected fluid (TOUGH2 variable $E X)$ or the time-dependent specific enthalpy of the produced or injected fluid (TOUGH2 variable $F 3(L), L T A B>1$ and ITAB non-blank). This parameter refers to a sink/source code name. Estimating a time-dependent enthalpy requires providing index $L$ through command $>>>>$ INDEX.

Note that enthalpy is also an observation type (see command $>>$ ENTHALPY (०)).

\section{Example}

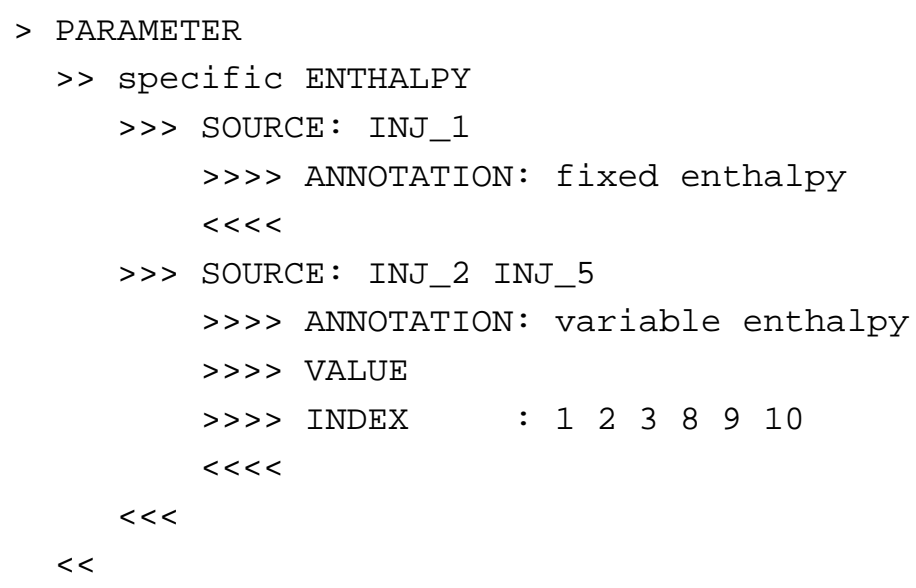

See Also

$>\operatorname{TIME}(\mathrm{p}),>>$ ENTHALPY (o) 


\section{Command}

>> ENTHALPY (phase_name/PHASE: iphase)

\section{Parent Command}

$>$ OBSERVATION

\section{Subcommand}

$>>$ SINK

\section{Description}

This command selects as an observation type the flowing enthalpy in a production well. This observation type refers to a sink code name. If multiple sinks are provided, the flowing enthalpy is weighted by the individual production rates. If a phase is selected either by specifying a valid phase_name or a phase number iphase or through command >>> P PHASE, only the flowing enthalpy of that phase is calculated.

Note that the enthalpy of the injected fluid can also be an unknown parameter to be estimated (see command >> ENTHALPY $(p)$ ).

\section{Example}

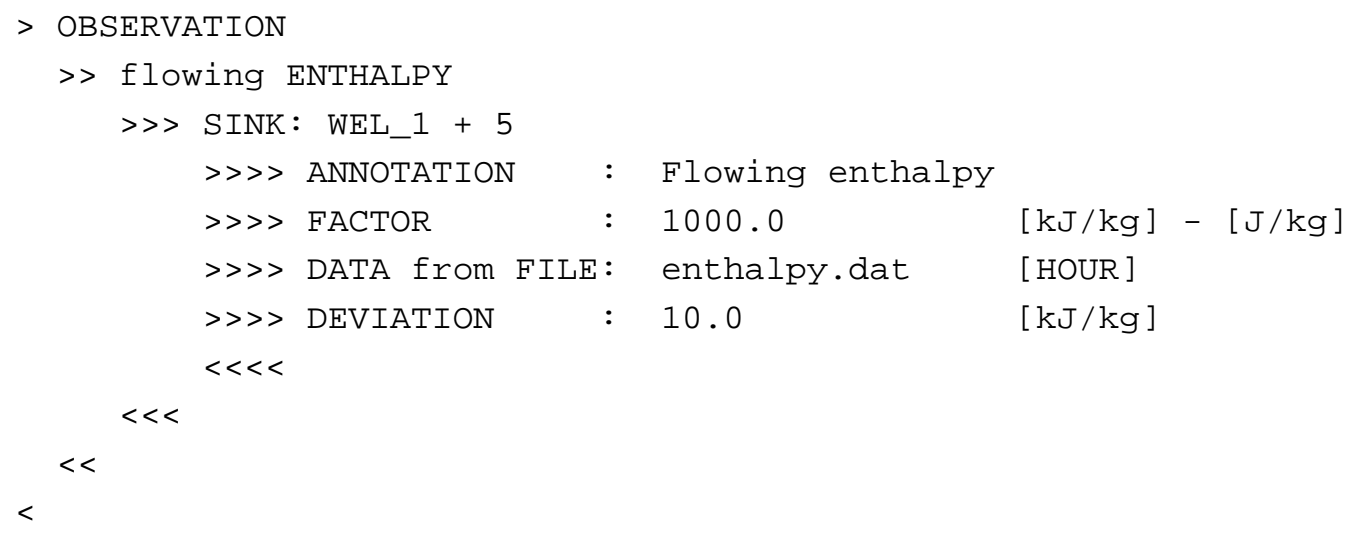

See Also

$>$ ENTHALPY (p) 


\section{Command}

>> EOF (MATRIX: ndim) (ITOUGH2) (CORRELATION)

\section{Parent Command}

$>$ ERROR

\section{Subcommand}

\section{Description}

(synonym for command $>>$ EMPIRICAL ORTHOGONAL FUNCTIONS)

\section{Example}

(see command >> EMPIRICAL ORTHOGONAL FUNCTIONS)

See Also

>> EMPIRICAL ORTHOGONAL FUNCTIONS 


\section{Command}

$>$ ERROR

\section{Parent Command}

$>$ COMPUTATION

\section{Subcommand}

$\gg>$ ALPHA

$>>$ EMPIRICAL ORTHOGONAL FUNCTIONS

$>>>$ FISHER

$>>$ FOSM

$\gg>$ HESSIAN

$>>>$ LINEARITY

$>>$ LIST

$>>$ MONTE CARLO

$>>$ POSTERIORI

$>>>$ PRIORI

$>>$ TAU

\section{Description}

This is the parent command of a number of subcommands that deal with the a posteriori error analysis or uncertainty propagation analysis.

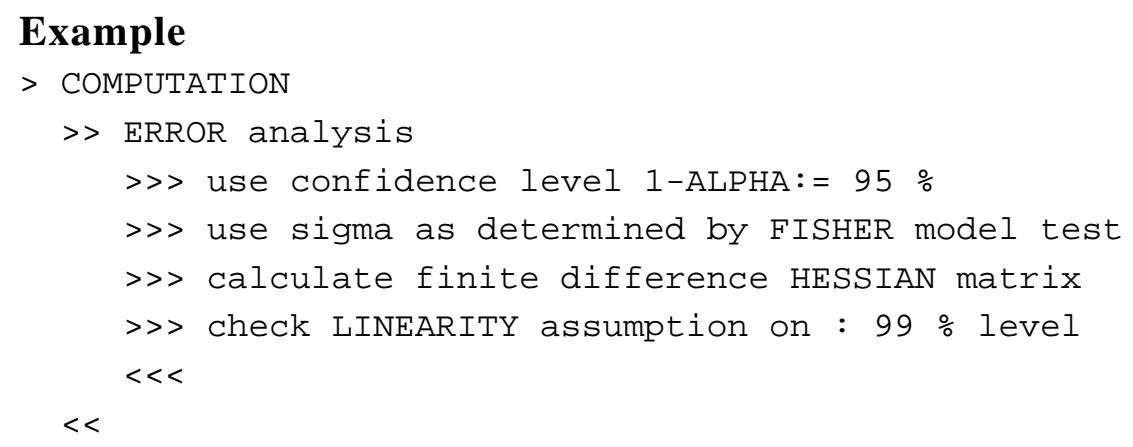

See Also 


\section{Command}

$>$ FACTOR

\section{Parent Command}

$>$ PARAMETER

\section{Subcommand}

$>>$ NONE

$>>\mathrm{SET}$

\section{Description}

This command selects as a parameter a constant factor with which the calculated TOUGH2 output will be multiplied. The factor is applied to the output that refers to a specific data set:

$$
z=z_{\text {TOUGH2 }} \cdot \text { factor }
$$

where factor is the multiplication factor and $z_{\text {TOUGH2 } 2}$ is the TOUGH2 output. The result $z$ is compared to the measurement $z^{*}$ of the corresponding data set.

This option allows correcting for a systematic, but unknown relative error in the data.

The data set is identified by number using command $>$ SET $(\mathrm{p})$.

If the factor is known and does not need to be estimated, use command

$>>>$ FACTOR (O).

\section{Example}

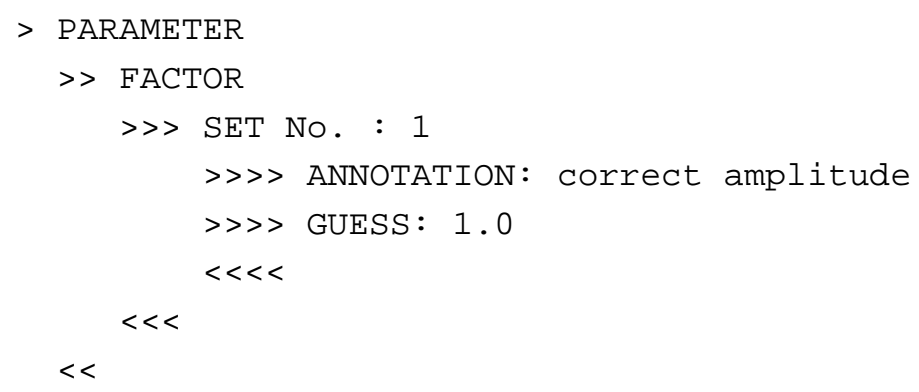

See Also

$>$ DRIFT, $>$ LAG, $>$ SHIFT, $>>>\operatorname{SET}(\mathrm{p}),>>>>$ FACTOR (o) 


\section{Command}

$>>>$ FACTOR

Parent Command

all third-level commands in block > PARAMETER

\section{Subcommand}

\section{Description}

The parameter to be estimated is a factor with which the initial TOUGH2 parameter value is multiplied:

$$
p=X / X_{0} \quad \Leftrightarrow \quad X=p \cdot X_{0}
$$

Here, $p$ is the estimated parameter, $X$ is the TOUGH2 parameter, and $X_{0}$ is the initial value of the TOUGH2 parameter. This option is useful to estimate a scaling factor for variable initial and boundary conditions, or to determine the mean of a quantity while maintaining ratios (e.g., if estimating a common factor applied to all three permeability values in a model domain, the anisotropy ratio remains constant). If the factor is log-normally distributed, add command $>>>>$ LOGARITHM or use command $>>>>$ LOG (F). Estimating a factor is opposed to estimating the parameter value directly (command $>>>>$ VALUE) or its logarithm (command >>> LOGARITHM (p)).

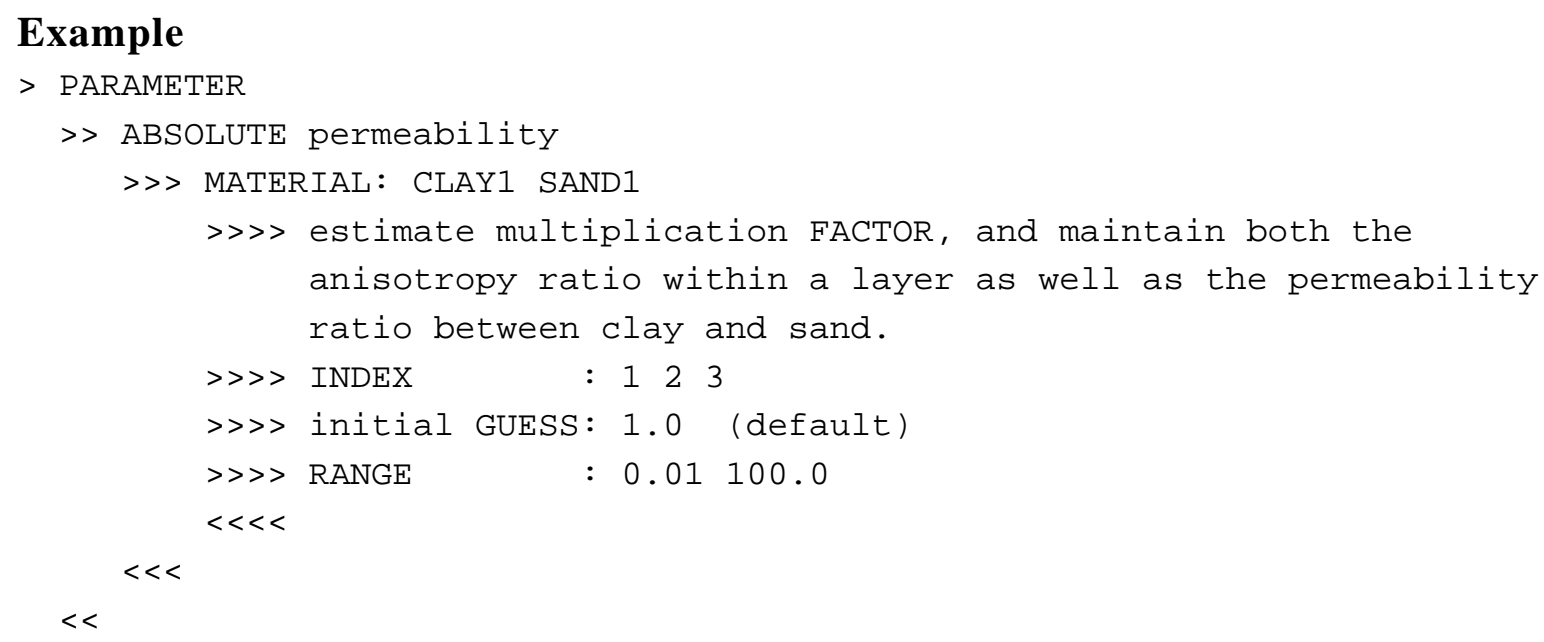

See Also

>>> LOGARITHM (p), >>> LOG $(\mathrm{F}), \quad$ > >> VALUE 


\section{Command}

>>> FACTOR: factor

\section{Parent Command}

all third-level commands in block > OBSERVATION

\section{Subcommand}

$-$

\section{Description}

This command provides a conversion factor with which the data are multiplied so they comply with standard TOUGH2 units. The conversion factor is also applied to the standard deviation (see command $>>>>$ DEVIATION (o)), i.e., the measurement error must be given in the same units as the data. The standard TOUGH2 units are used throughout the iTOUGH2 output file, i.e., all observed and calculated values as well as residuals and standard deviations have been multiplied by factor.

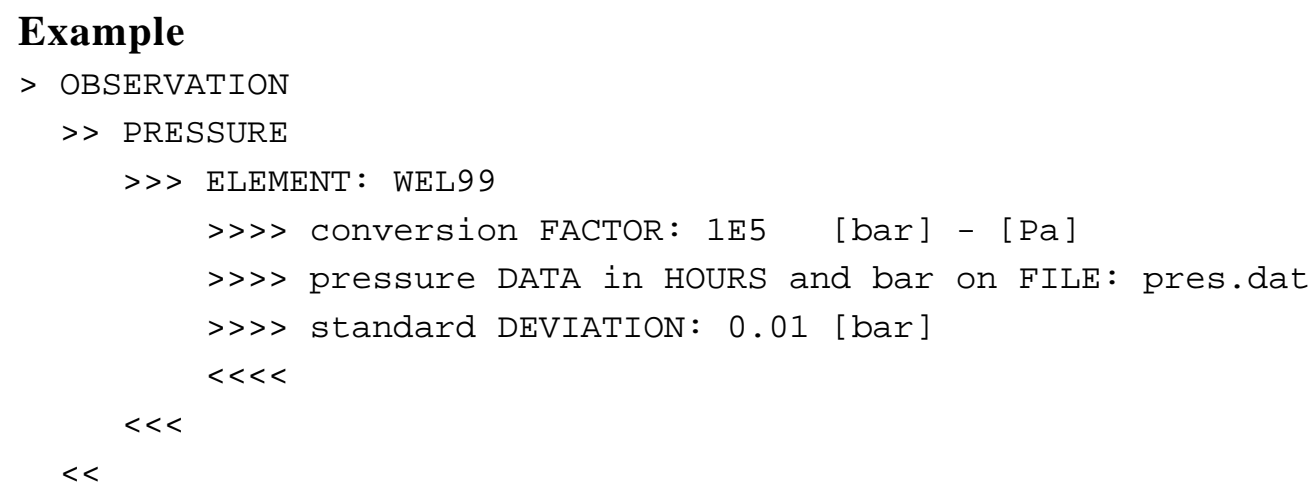

See Also

$>$ FACTOR 


\section{Command}

>> FISHER

\section{Parent Command}

$>$ ERROR

\section{Subcommand}

\section{Description}

The a posteriori estimated error variance $s_{0}^{2}$ represents the variance of the mean weighted residual and is thus a measure of goodness-of-fit:

$$
s_{0}^{2}=\frac{\mathbf{r}^{T} \mathbf{C}_{z z}^{-1} \mathbf{r}}{m-n}
$$

The value $s_{0}^{2}$ is used in the subsequent error analysis. For example, the covariance matrix of the estimated parameters, $\mathbf{C}_{p p}$, is directly proportional to the scalar $s_{0}$. Note that if the residuals are consistent with the distributional assumption about the measurement errors (i.e., matrix $\mathbf{C}_{z z}$ ), then the estimated error variance assumes a value close to one. $s_{0_{2}}^{2}$ is also an estimate for the true or a priori error variance $\sigma_{0}^{2}$. It can be shown that the ratio $s_{0} / \sigma_{0}^{2}$ follows an $F$-distribution with the two degrees of freedom $f_{1}=m-n$, and $f_{2}=\infty$. Therefore, it can be statistically tested to see whether the final match deviates significantly from the modeler's expectations, expressed by matrix $\mathbf{C}_{z z}$. This is called the Fisher Model Test. The user must decide whether the error analysis should be based on the a posteriori or a priori error variance (see commands $>>>$ POSTERIOR and $>>>$ PRIOR, respectively). The decision can also be delegated to the Fisher Model Test according to the following table:

\begin{tabular}{ccl}
\hline Fisher Model Test & Error Variance & Comment \\
\hline$s_{0}^{2} / \sigma_{0}^{2}>F_{m-n, \infty, 1-\alpha}$ & $s_{0}^{2}$ & Error in functional and/or stochastic model \\
$1 \leq s_{0}^{2} / \sigma_{0}^{2} \leq F_{m-n, \infty, 1-\alpha}$ & $s_{0}^{2}$ & Model test passed \\
$s_{0}^{2} / \sigma_{0}^{2}<1$ & $\sigma_{0}^{2}$ & Error in stochastic model \\
\hline
\end{tabular}

\section{Example}

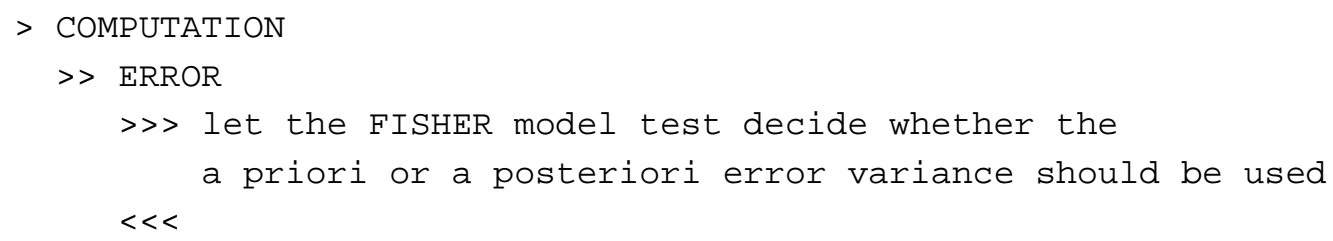

See Also

>> ALPHA, >> POSTERIORI, >> PRIORI 


\section{Command}

>> FLOW (phase_name/PHASE: iphase/HEAT)

Parent Command

$>$ OBSERVATION

\section{Subcommand}

>> CONNECTION

\section{Description}

This command selects as an observation type the flow rate of phase iphase or total fluid flow rate or heat flux. This observation type refers to a connection.

The phase name phase_name or phase number iphase, which depend on the EOS module being used, are listed in the iTOUGH2 header. They can be specified either on the command line or using the subcommand >>>> PHASE. If no phase is specified, the total flow rate is selected. If keyword HEAT is present, the total heat flux across the connection is selected. Note that the sign of the calculated flow rate depends on the order of the two elements in a connection.

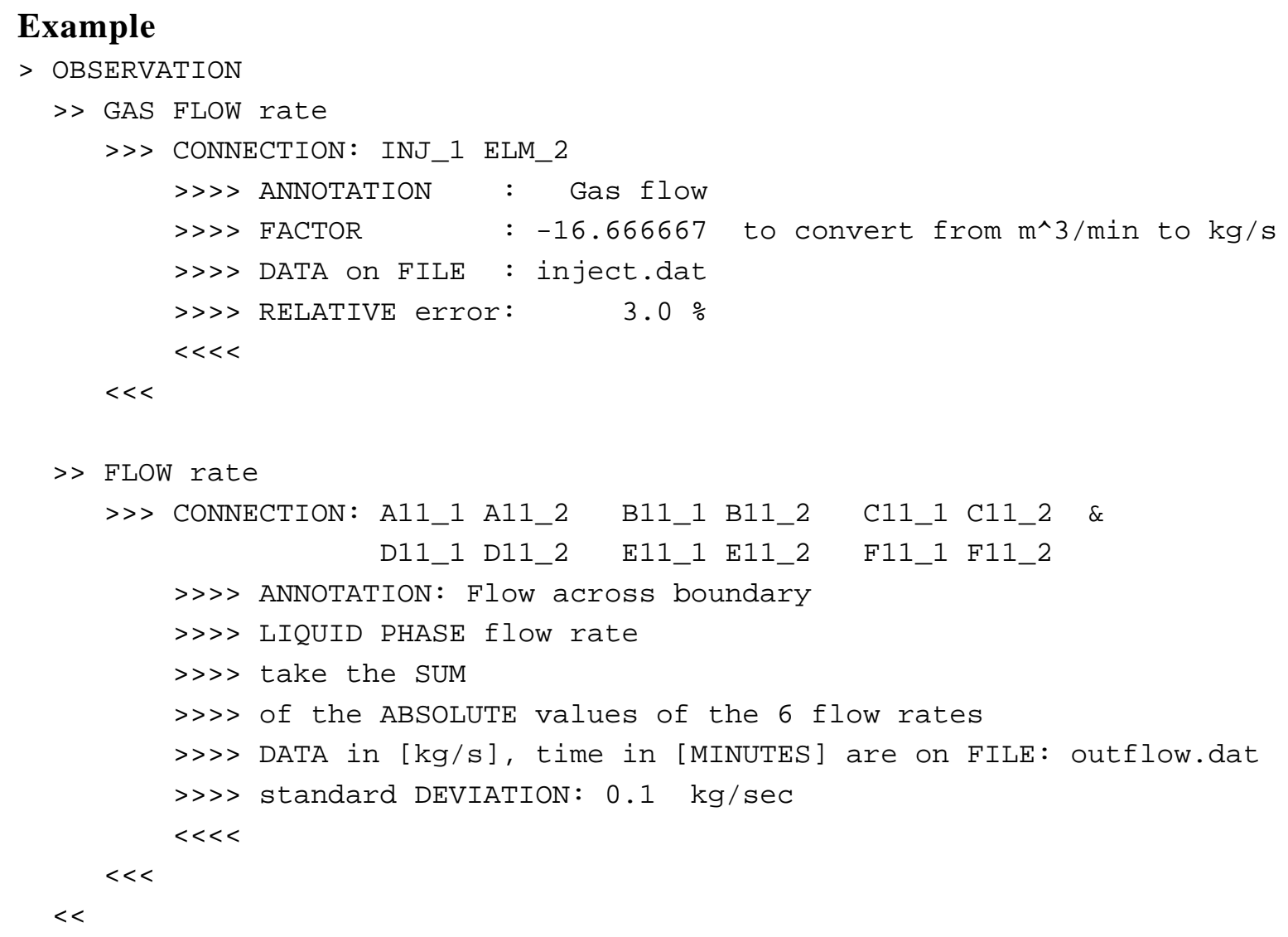

See Also

$-$ 


\section{Command}

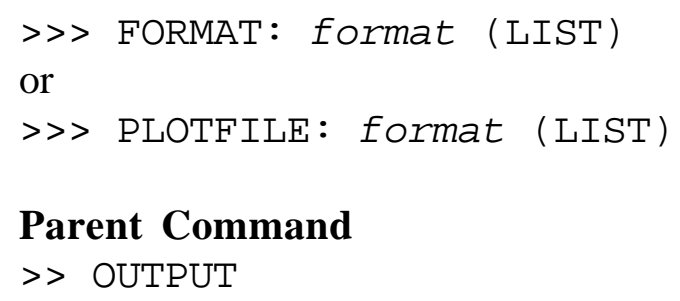

\section{Subcommand}

\section{Description}

iTOUGH2 does not directly generate graphs (except for the residual plot and correlation chart). However, it does generate a plot file with data at the calibration points and system response as calculated with the initial, intermediate (see command >>> PLOTTING), and final parameter set. A plot file with the relative permeability and capillary pressure curves can also be requested (see command >> CHARACTERISTIC). These plot files must be processed by an external visualization package. iTOUGH2 generates plot files in PLOPO format, a visualization software developed by U. Kuhlmann at ETH, Zürich. The PLOPO plot file is internally reformatted to comply with formats of other visualization programs. The string format identifies the visualization software (for a list of available formats add keyword LIST). The reformatted plot files have a file extension specific for the chosen software (for example ".tec" for TECPLOT.) A general format accepted by most commercially available plotting programs is the arrangement in columns, where the first column contains the time, and additional columns hold the data and calculated system response for various observations. This general format can be obtained by using keyword COLUMN.

The default format is TECPLOT, and can be changed to another format in file it2main.f, BLOCK DATA IT, through variable IPLOTFMT. Additional interfaces can be programmed into subroutine PLOTIF and REFORMAT.

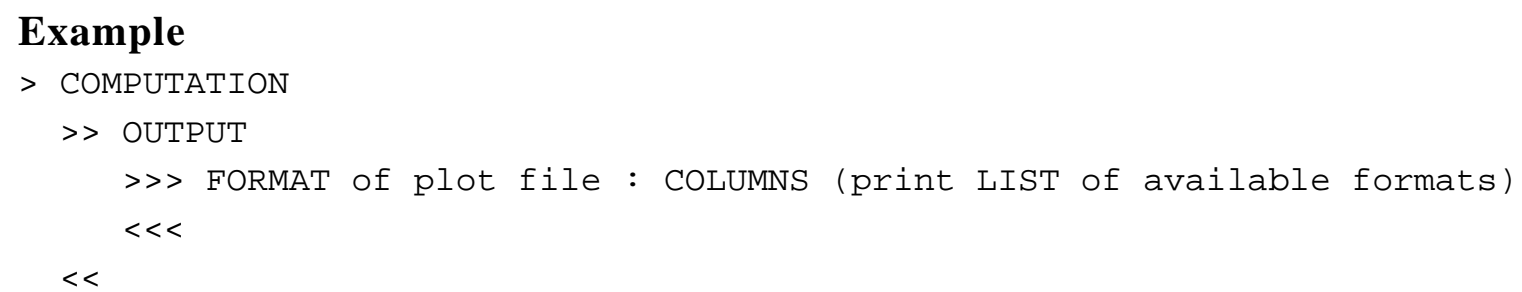

See Also

>> CHARACTERISTIC, $>>>$ PLOTTING 


\section{Command}

>>> FORMAT: format

Parent Command

all third-level commands in block > OBSERVATION

\section{Subcommand}

\section{Description}

This command accepts a FORTRAN format statement for reading data. By default, data are read in free format following the >>>> DATA command or directly from a data file. If a column contains non-numeric characters, formatted input can be invoked by providing a string format representing the corresponding FORTRAN format statement. The format statement must be in brackets and must not contain any blanks.

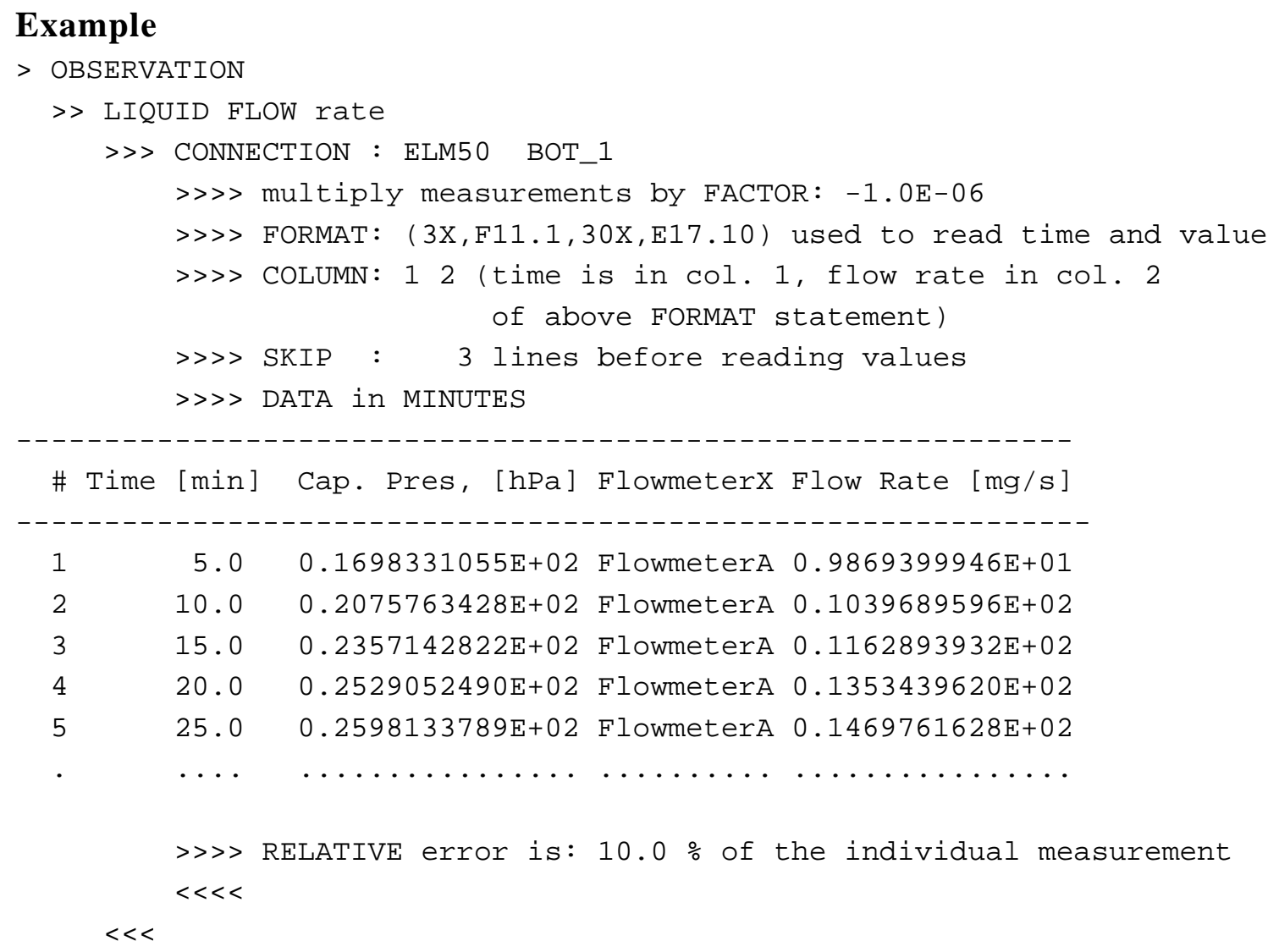




\section{Command}

$>>$ FORWARD

or

$>>$ DIRECT

Parent Command

>> CONVERGE

or

$>$ OPTION

\section{Subcommand}

$-$

\section{Description}

This command allows one TOUGH2 simulation to be performed in order to solve the forward problem.

It is advantageous to perform a single TOUGH2 simulation before invoking more expensive inversions. The result from the forward run can be used to check TOUGH2 and iTOUGH2 input. Furthermore, a plotfile is generated with the results from the simulation with the initial parameter set which can be compared to the observed data. The CPU time requirement for an inversion can also be estimated from a single TOUGH2 simulation.

(Note that there is another command $>>>$ FORWARD in block >> JACOBIAN.)

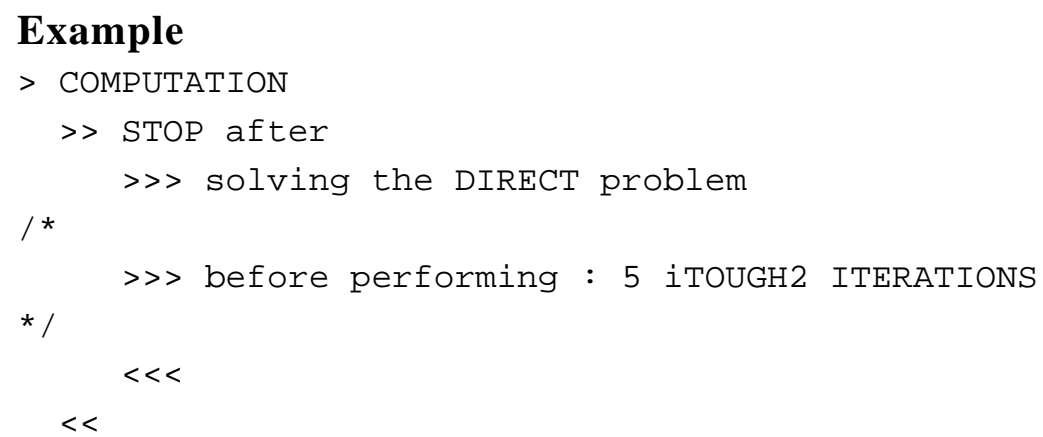

See Also

>> SIMULATION 


\section{Command}

>> FORWARD (: iswitch)

\section{Parent Command}

$>>$ JACOBIAN

\section{Subcommand}

$-$

\section{Description}

With this command the elements of Jacobian matrix are calculated by means of a forward finite difference quotient:

$$
J_{i j}=\frac{\partial z_{i}}{\partial p_{j}} \approx \frac{z_{i}\left(\mathbf{p} ; p_{j}+\delta p_{j}\right)-z_{i}(\mathbf{p})}{\delta p_{j}}
$$

The evaluation of the Jacobian thus requires $n+1$ TOUGH 2 simulations, where $n$ is the number of parameters. The size of the perturbation $\delta p_{j}$ can be controlled using command $>>$ PERTURB.

The Jacobian is used in both the minimization algorithm and the a posteriori error analysis. For the Levenberg-Marquardt minimization algorithm, the accuracy obtained by using a forward (as opposed to centered) finite difference quotient is usually sufficient, especially during the first few iterations far away from the minimum. However, if approaching the minimum and especially for the subsequent error analysis one might want to use a more accurate approximation of the Jacobian. If a colon is given on the command line followed by an integer iswitch, iTOUGH2 switches from forward to centered finite differences after iswitch iterations.

\section{Example}

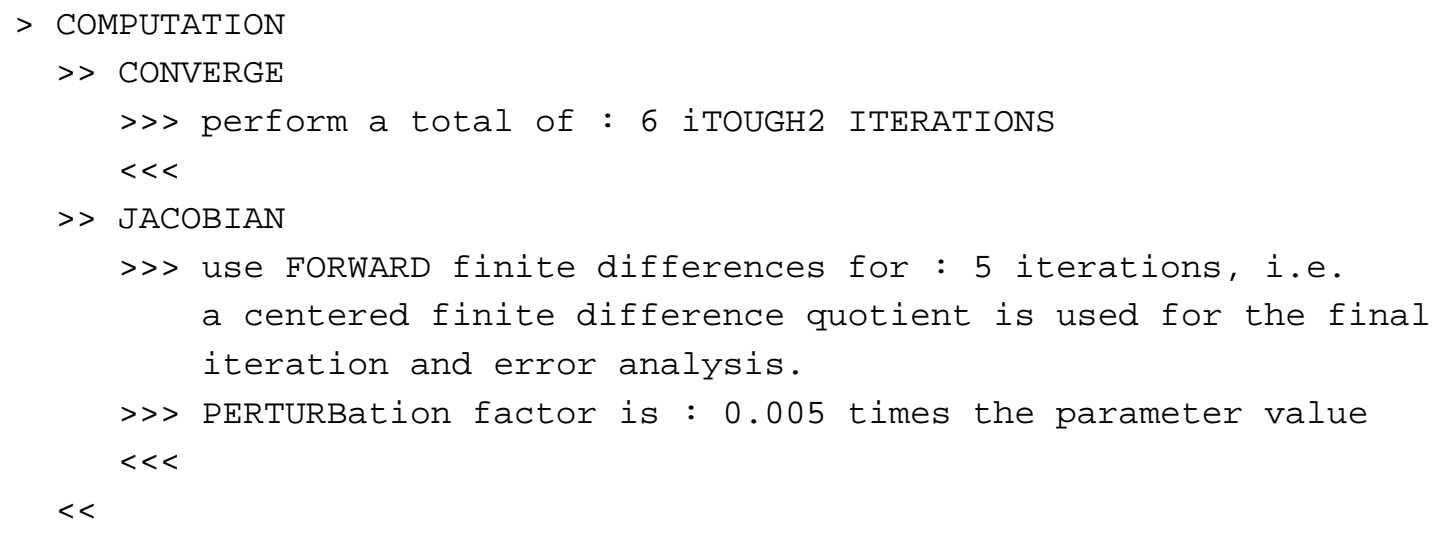

See Also

>>> CENTERED, >>> PERTURB 


\section{Command}

>> FOSM (MATRIX: ndim) (iTOUGH2) (CORRELATION) (DIAGONAL)

\section{Parent Command}

$>$ ERROR

\section{Subcommand}

\section{Description}

This command performs First-Order-Second-Moment (FOSM) uncertainty propagation analysis. FOSM quantifies the uncertainty of model predictions as result of parameter uncertainty. FOSM is the analysis of the mean and covariance of a random function based on its first order Taylor series expansion. FOSM analysis presumes that the mean and covariance are sufficient to characterize the distribution of the dependent variables, i.e., the model results are assumed to be normally distributed, and perturbations about the mean can be approximated by linear functions $\mathbf{J}$. The covariance of the uncertain parameters, $\mathbf{C}_{p p}$, is translated into the covariance of the simulated system response, $\mathbf{C}_{\hat{z} \hat{z}}$ :

$$
\mathbf{C}_{\hat{z} \hat{z}}=\mathbf{J C}_{p p} \mathbf{J}^{T}
$$

The diagonal elements of matrix $\mathbf{C}_{p p}$, i.e., the variances of the parameters, can be supplied by command $>>>>$ VARIANCE (or related commands) in block > PARAMETER. If correlations are to be taken into account, the full covariance matrix must be provided. The elements of matrix $\mathbf{C}_{p p}$ can be supplied using one of the following options:

(1) Provide indices and elements of $\mathbf{C}_{p p}$. Example:

>> FOSM error analysis

$110.80643 \mathrm{E}-04$

$220.71921 \mathrm{E}-04$

$210.64412 \mathrm{E}-04$

Use keyword CORRELATION if off-diagonal term is correlation coefficient instead of covariance. Example:

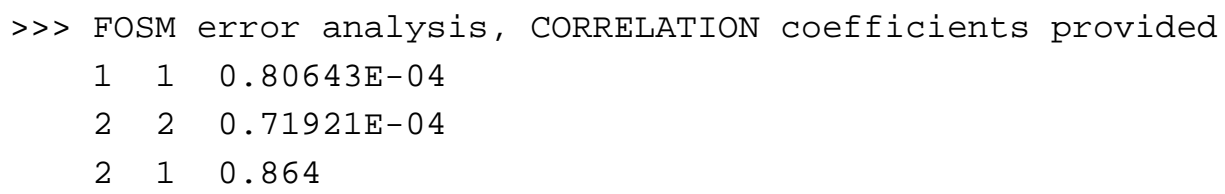

(2) Provide keyword MATRIX, followed by a colon and the dimension ndim of the square matrix $\mathbf{C}_{p p}$. The lower triangle of the covariance matrix is then provided on exactly ndim additional lines. If keyword CORRELATION is present, the offdiagonal terms represent correlation coefficients rather than covariances. Example: 


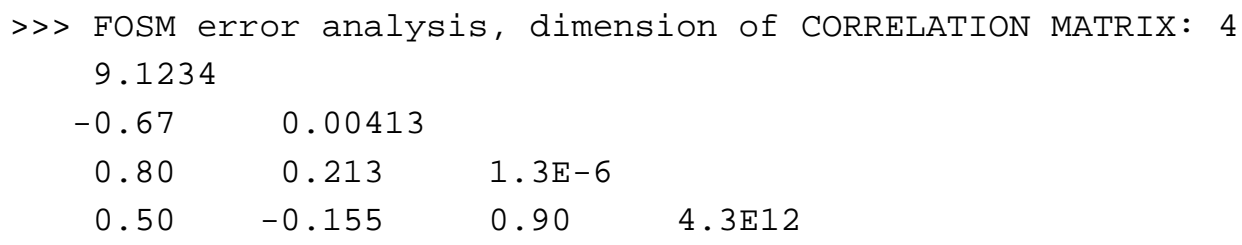

(3) If calculated during a previous iTOUGH2 inversion, the covariance matrix can be taken from the iTOUGH2 output file and directly copied after the command line. This option is invoked by keyword iTOUGH2. The matrix will be read by formatted input, so it is crucial that the correct format is maintained. If ndim is greater than 6 , the matrix is split in multiple submatrices. All submatrices must be copied exactly as they were printed to the iTOUGH2 output file. Example:

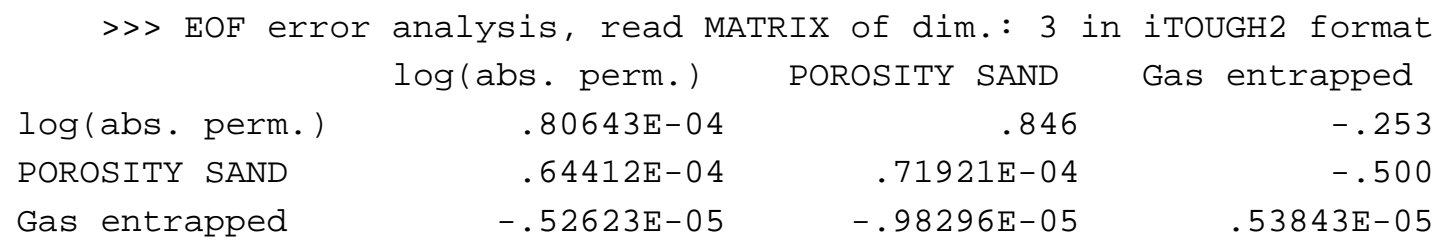

If the full matrix is provided, but only the diagonal terms (variances) shall be used in the error analysis, use keyword DIAGONAL on the command line. This option makes it easy to study the impact of correlations on the uncertainty propagation analysis.

The uncertainty of the model prediction as a result of parameter uncertainty is given as a standard deviation in the residual analysis. Furthermore, the plotfile contains the system response for the mean parameter set as well as error band on the specified confidence level (see command $>>>$ ALPHA).

It is suggested to also increase the perturbation factor for calculating the Jacobian matrix, and to use a centered finite difference quotient. This yields in an more realistic the error band if the model is highly non-linear. It should be realized, however, that Monte Carlo is the preferred method if dealing with highly non-linear flow systems.

\section{Example}

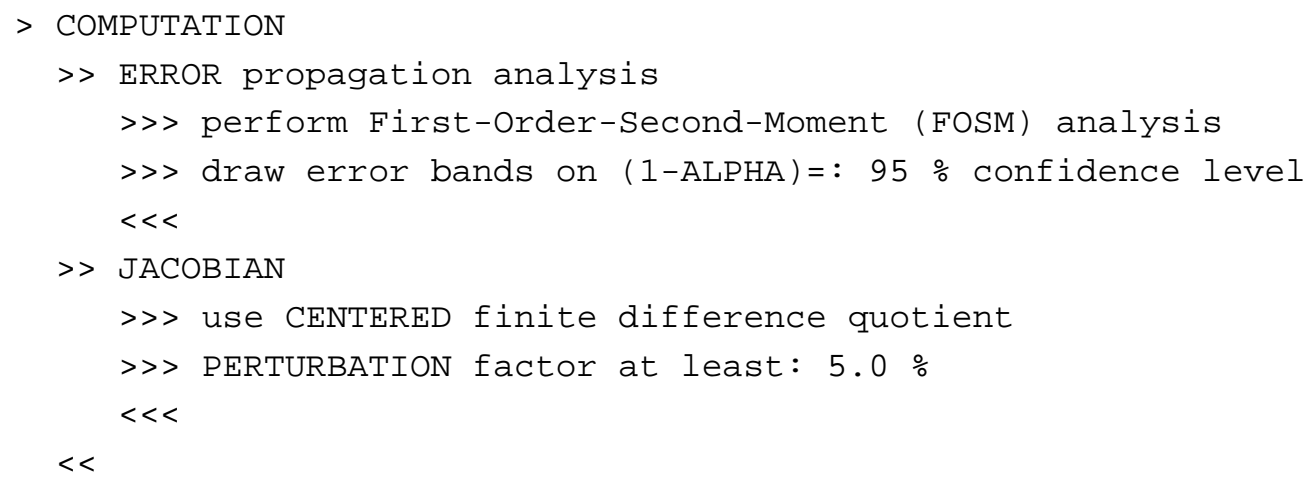

See Also

>> ALPHA, >> EMPIRICAL ORTHOGONAL FUNCTIONS, >> MONTE CARLO 


\section{Command}

$>>>$ GAUSS

or

>>> NORMAL

Parent Command

all third-level commands in block > PARAMETER

\section{Subcommand}

\section{$-$}

\section{Description}

This command generates normally distributed input parameters for Monte Carlo simulations. This is the default distribution. Parameter values will be generated following a normal distribution with the initial guess as the mean, and the standard deviation taken from command $>>>>$ DEVIATION. Only values within the specified range will be accepted. If command $>>>>$ LOGARITHM is also present, parameters follow a log-normal distribution.

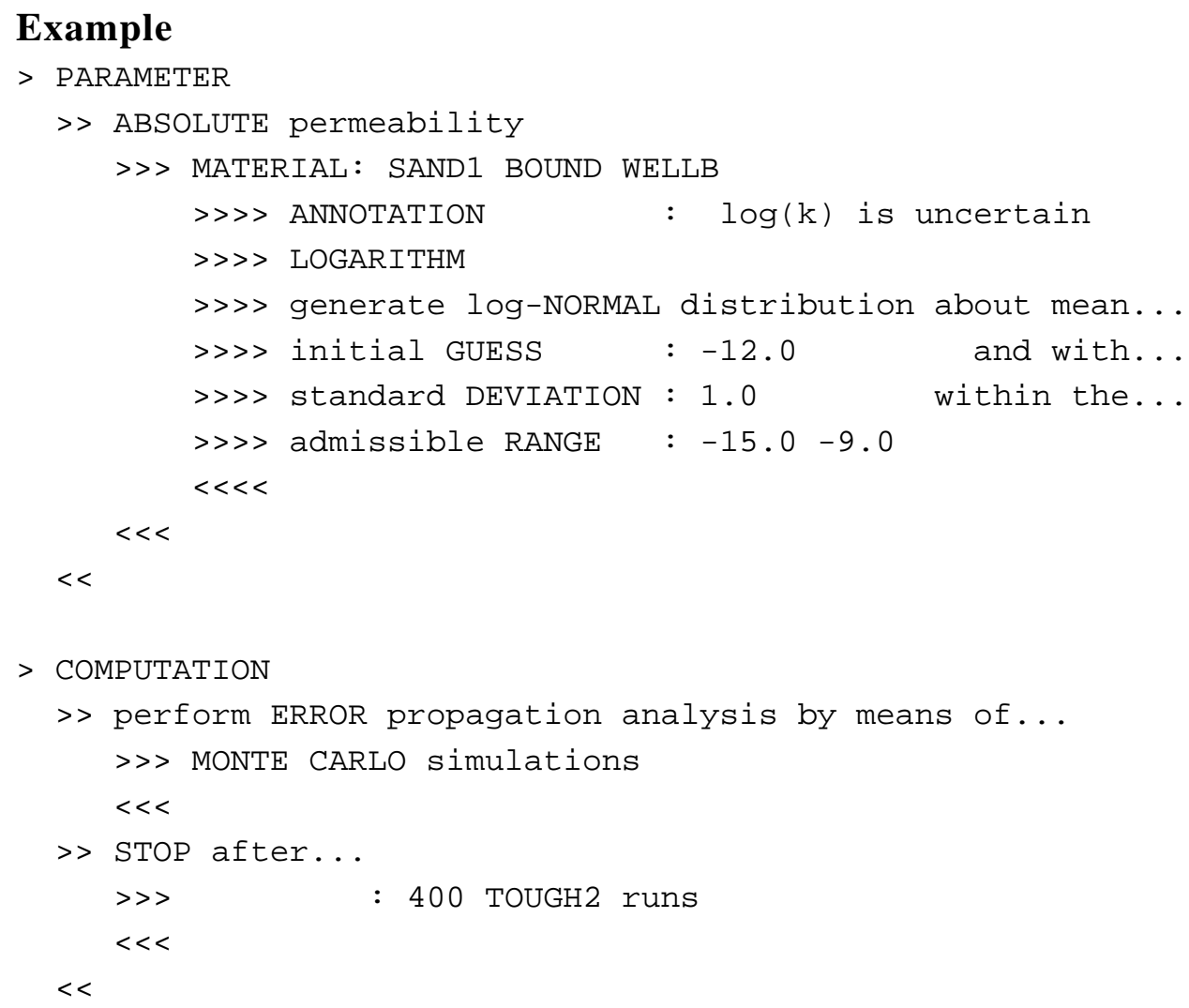

See Also

$>>$ MONTE CARLO, $>>>$ UNIFORM 


\section{Command}

>> GAUSS-NEWTON

\section{Parent Command}

$>$ OPTION

\section{Subcommand}

$-$

\section{Description}

This command performs Gauss-Newton steps to minimize the objective function. The Gauss-Newton algorithm assumes linearity and can be described as follows:

$$
\begin{gathered}
\Delta \mathbf{p}=\left(\mathbf{J}^{T} \mathbf{C}_{z z}^{-1} \mathbf{J}\right)^{-1} \mathbf{J}^{T} \mathbf{C}_{z z}^{-1} \mathbf{r} \\
\mathbf{p}^{(k+1)}=\mathbf{p}^{(k)}+\Delta \mathbf{p}
\end{gathered}
$$

Gauss-Newton steps are efficient if the model is linear (only one iteration required to find minimum) or nearly-linear. If the model is highly nonlinear, Gauss-Newton steps are usually too large, leading to an inefficient or even unsuccessful step.

By default, iTOUGH2 uses the Levenberg-Marquardt minimization algorithm, which is a modification of the Gauss-Newton algorithm.

\section{Example}

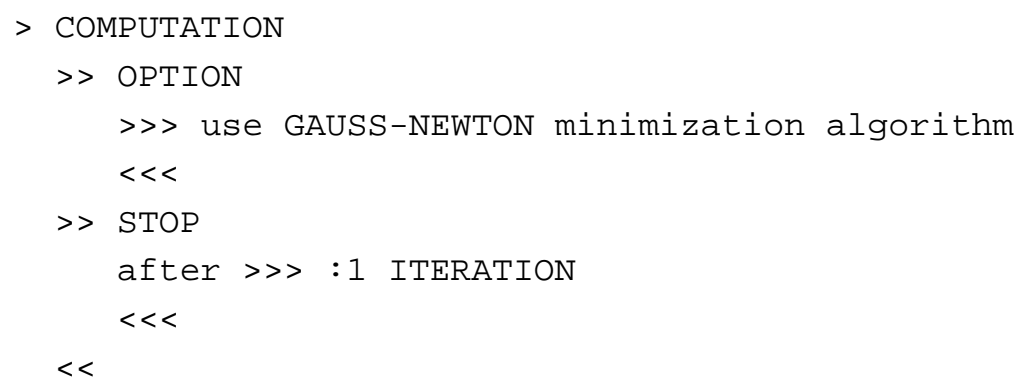

See Also

$>>$ ANNEAL, $>>>$ GRID SEARCH, $>>>$ LEVENBERG-MARQUARDT, $>>$ SIMPLEX 


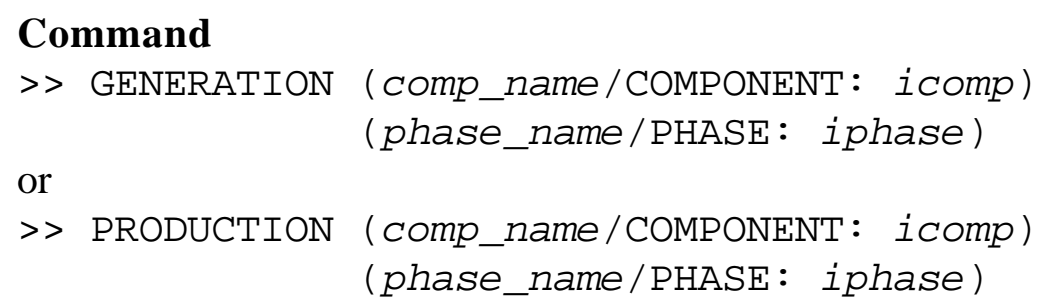

\section{Parent Command}

$>$ OBSERVATION

\section{Subcommand}

$>>$ SINK

\section{Description}

This command selects as an observation type the total or fractional generation rate in a production well. This observation type refers to a sink code name.

The total generation rate is usually prescribed in TOUGH2 block GENER, or can be considered a parameter to be estimated (see command $>>$ RATE). A variable generation rate suitable for calibration is obtained only for wells on deliverability (type DELV), or if the generation rate of a specific phase or component is used.

The fractional generation rate refers to the production of a specific phase or component. The component name comp_name or component number icomp, as well as the phase name phase_name or phase number iphase, which depend on the EOS module being used, are listed in the iTOUGH2 header. They can be specified either on the command line or using the subcommands >>>> COMPONENT and >>>> PHASE, respectively. If neither a phase nor a component is specified, the total generation rate is calculated. If a phase but no component is selected, the generation rate of that phase including all components is calculated. If a component but no phase is selected, the generation rate of that component in all phases is calculated. Finally, if a specific phase and a specific component is given, only the generation rate of that component in the specified phase is calculated.

\section{Example}

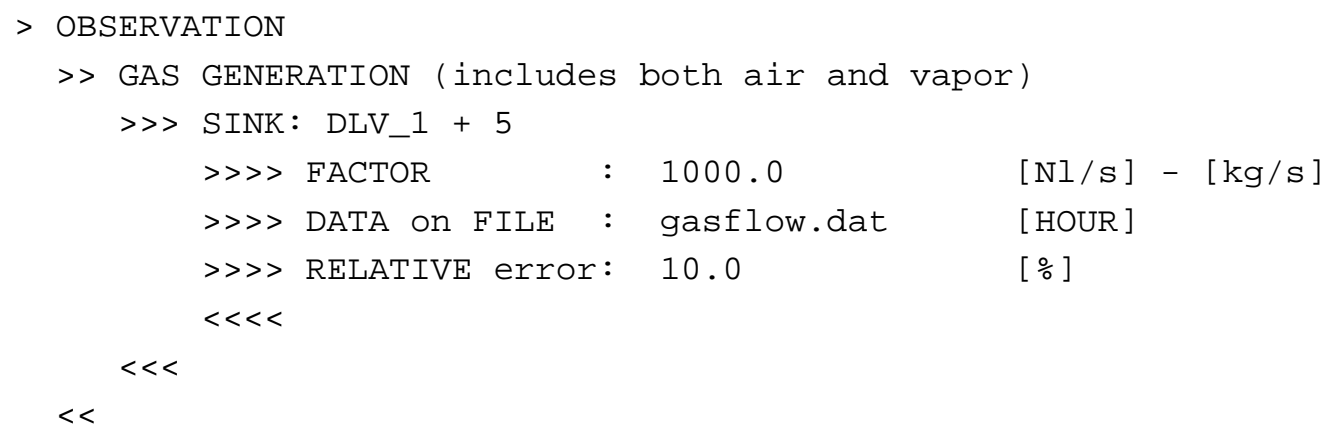

See Also

$>$ CUMULATIVE, $>$ RATE, > TOTAL MASS, >>> COMPONENT, $>>>$ PHASE 


\section{Command}

>> GRID BLOCK: eleme (eleme_i ...) (+ iplus)

\section{Parent Command}

all second-level commands in block > OBSERVATION requiring element names.

Subcommand

all fourth-level commands in block > OBSERVATION

Description

(synonym for command >>> ELEMENT)

\section{Example}

(see command $>>$ ELEMENT)

See Also

$>>$ ELEMENT 


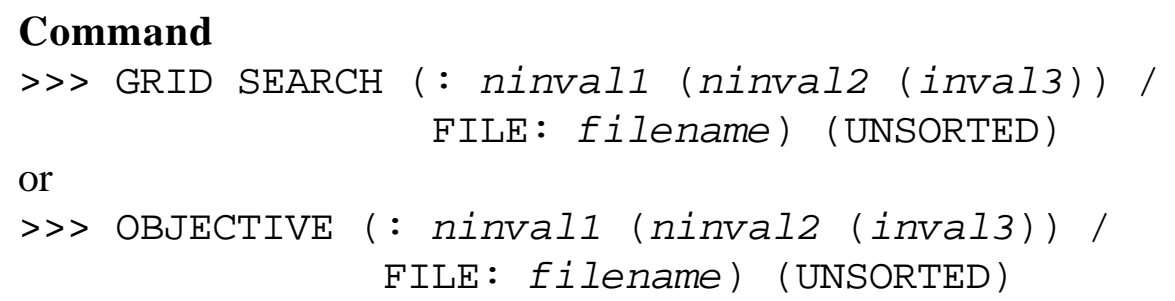

Subcommand

$-$

\section{Description}

This command evaluates the objective function for a number of specific parameter sets. A list of parameter sets can be provided on file filename. Alternatively, parameter sets are internally generated on a regular grid, mapping out the entire parameter space. In this case, lower and upper bounds must be defined for each parameter using command $>>>>$ RANGE in block $>$ PARAMETER. This range is then subdivided into ninteri intervals by inserting invali+1 equally spaced points, generating parameter sets on a regular grid in the $n$ dimensional parameter space. The objective function is evaluated at each grid point. Keyword UNSORTED may be used in PVM applications to improve efficiency. Evaluating the objective function throughout the entire parameter space is referred to as grid searching. The parameter set, the value of the objective function, and the contribution of each observation type to the objective function is printed to the iTOUGH2 output file. The global minimum is likely to be in the vicinity of the parameter set with the lowest objective function. Furthermore, the information listed in the output file can be used to visually represent and study the topology of the objective function. For example, one can generate a contour plot of the objective function for $n=2$ which may reveal the presence local minima. If only one output variable is defined in block > OBSERVATION and no data are provided, this option can also be used in combination with command $>>>$ L1-ESTIMATOR to examine the sensitivity of the output variable over an extensive parameter range.

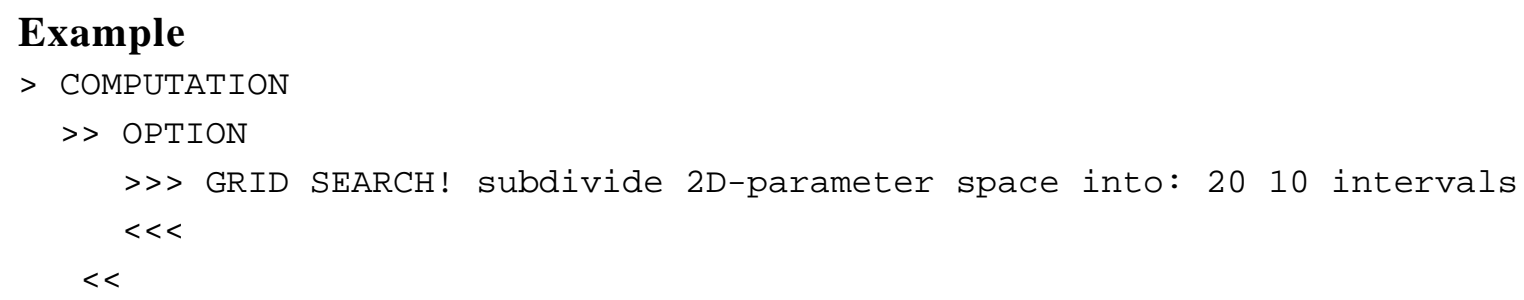

See Also

>>> ANNEAL, >>> GAUSS-NEWTON, >>> LEVENBERG-MARQUARDT, >>> PVM, 


\section{Command}

>> GUESS (FILE: file_name)

\section{Parent Command}

$>$ PARAMETER

\section{Subcommand}

\section{Description}

This command identifies initial guesses of the parameters to be estimated. The initial guess vector $\mathbf{p}^{(k=0)}=\mathbf{p}_{0}$ is the starting point of the minimization algorithm (iteration $k=0$ ). Usually, the initial guess vector is identical with vector $\mathbf{p} *$ which holds prior information about the parameters. By default, $\mathbf{p}_{0}$ and $\mathbf{p} *$ are identical, taken from the TOUGH2 input file, and overwritten by command $>>>>$ PRIOR. The starting point for the minimization algorithm may be different from the prior information vector. In this case, prior information is taken from the TOUGH 2 input file or must be provided through command $>>>>$ PRIOR. The starting point is taken from command $>$ GUESS which overwrites the initial guess provided through command $>>>>$ GUESS.

A parameter is identified by an integer value indicating its position in the parameter block:

\section{XIGUESS(I)}

This format is identical to that of file <invfile>.par. Initial guesses can be provided either following the command line, or read from a file if keyword FILE is present. The filename is given after the colon. This latter option is useful to transfer best estimates from one inversion to another if the order of the parameters is the same.

\section{Example}

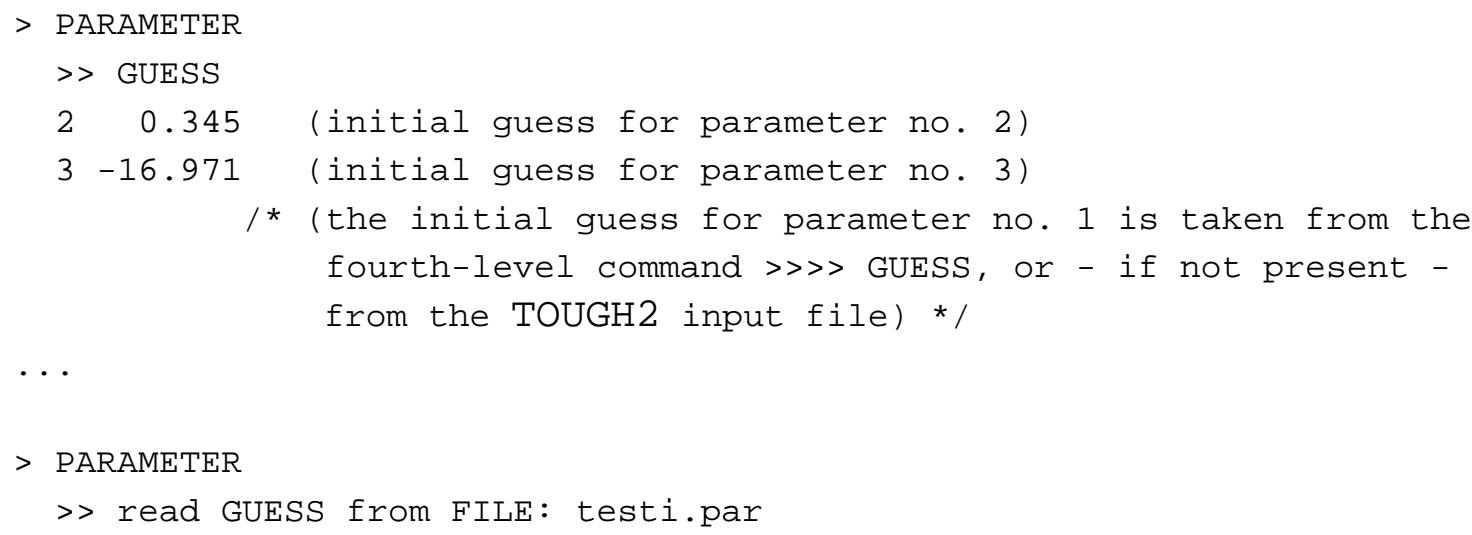

See Also

>>> GUESS, $>>>>$ PRIOR 


\section{Command}

>>> GUESS: guess

\section{Parent Command}

all third-level commands in block > PARAMETER

\section{Subcommand}

$-$

\section{Description}

This command provides an initial guess of the parameter to be estimated. If neither this command nor command > GUESS is used, the initial guess is taken from the TOUGH2 input file. The initial guess is the starting point for the minimization algorithm, to be distinguished from prior information (see command $>>>>$ PRIOR). The initial guess can be overwritten by the second-level command $>>$ GUESS.

If command $>>>>$ LOGARITHM is present, the initial guess is the logarithm of the parameter. Similarly, if command $>>>>$ FACTOR is present, the initial guess should be a multiplication factor (default is 1.0).

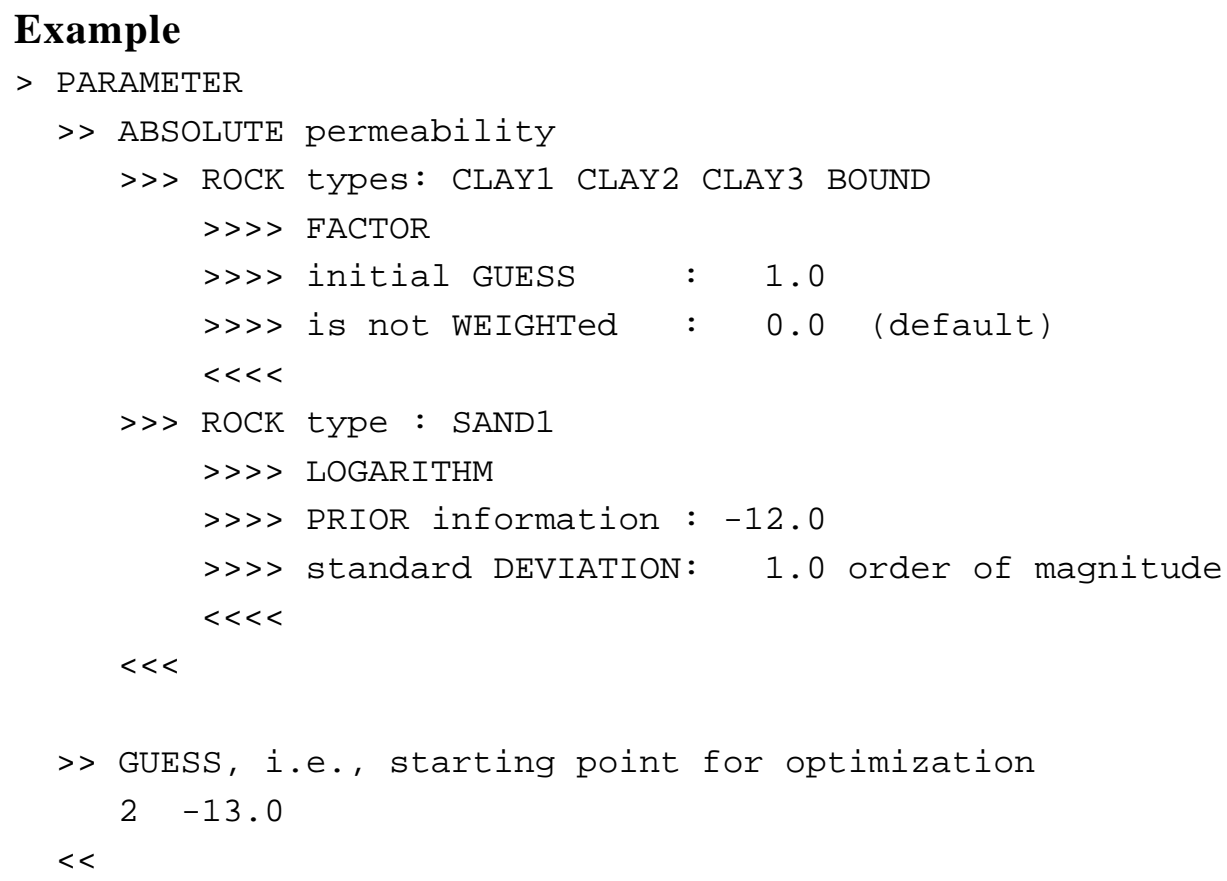

See Also

$>$ GUESS, $>>>$ PRIOR, $>>>$ DEVIATION, $>>>$ V VARIATION 


\section{Command}

>>> HEADER: nskip

or

>>> SKIP: nskip

Parent Command

all third-level commands in block > OBSERVATION

\section{Subcommand}

\section{$-$}

\section{Description}

This command identifies the number of lines to be skipped before reading data. Data reading starts nskip+1 lines after the command line >>>> DATA, or on the (nskip+1)-th line of the data file (see command $>>>>$ DATA). Lines containing data are skipped using command >>> P PICK.

\section{Example}

$>$ OBSERVATION

$>>$ TEMPERATURE

>> ELEMENTS: ELM_10 + 4

>>> SKIP: 3 lines before reading data

$>>>$ DATA [HOURS ]

(1)

(2)

-------------------------------------------------------------

(3)

$\begin{array}{lll}0.00 & 21.3 & \text { mean temperature prior to experiment } \\ 1.12 & 21.3 \\ 1.15 & 21.9 \\ 1.20 & 23.4 \\ 2.00 & 32.8 \\ 2.10 & 29.3 \\ 2.20 & 26.7 \\ 2.30 & 24.1 \\ 4.00 & 21.6 & \\ & \end{array}$

$>>>$ standard DEVIATION: 0.5 degrees C $<<<$

$<<<$

$<<$

See Also

$>>>$ COLUMN, $>>>>$ DATA, $>>>$ FORMAT, $>>>>$ PICK, $>>>>$ SET (o) 


\section{Command}

HELP (a keyword in combination with any command)

\section{Parent Command}

\section{Subcommand}

$-$

\section{Description}

A short message about the command usage is printed to the iTOUGH2 output file if keyword HELP is present on the command line. See also LIST and $>>>$ INDEX for further support. If command $>>>$ INPUT is used, the help message can be retrieved without performing any iTOUGH2 calculations.

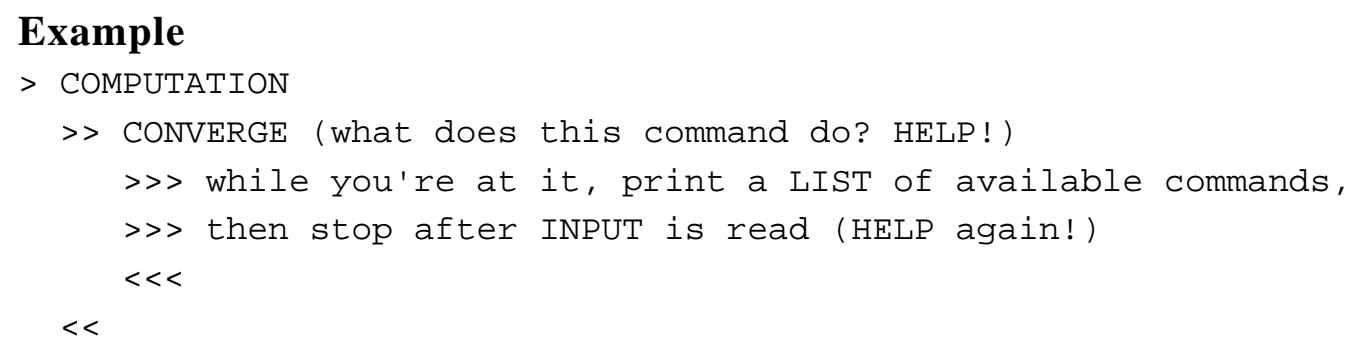

See Also

>> INDEX, LIST 


\section{Command}

$>>$ HESSIAN

\section{Parent Command \\ $>$ ERROR \\ $>>$ JACOBIAN}

\section{Subcommand}

\section{Description}

This command computes a finite difference Hessian matrix $\mathbf{H}$ for the error analysis following optimization. The elements of $\mathbf{H}$ are given by:

$$
H_{j k}=2 \sum_{i=1}^{m} \frac{1}{\sigma_{i}^{2}}\left[\frac{\partial z_{i}}{\partial p_{j}} \frac{\partial z_{i}}{\partial p_{k}}-r_{i} \frac{\partial^{2} z_{i}}{\partial p_{j} \partial p_{k}}\right]
$$

The evaluation of $\mathbf{H}$ by means of finite differences requires $2 n+n(n-1) / 2$ additional TOUGH2 simulations, where $n$ is the number of parameters. By default, the Hessian matrix, which is the inverse of the parameter covariance matrix, is approximated by $\mathbf{J}^{T} \mathbf{C}_{z z}^{-1} \mathbf{J}$ based on the linearity assumption, i.e., the second derivative term is ignored. Evaluating the finite difference Hessian, which takes into account the non-linearities, provides a means by which to check the linearity assumption (for another approach see command >> LINEARITY). This may lead to a more accurate calculation of the covariance matrix of the estimated parameters. However, inclusion of the second-derivative term may yield a Hessian matrix that is not positive definite due to the presence of outliers, strong nonlinearities, or the fact that the minimum has not been detected accurately, i.e., when the positive and negative residuals $r_{i}$ do not cancel each other. In this case, iTOUGH2 automatically proceeds with the linearized Hessian which is positive definite by definition.

\section{Example}

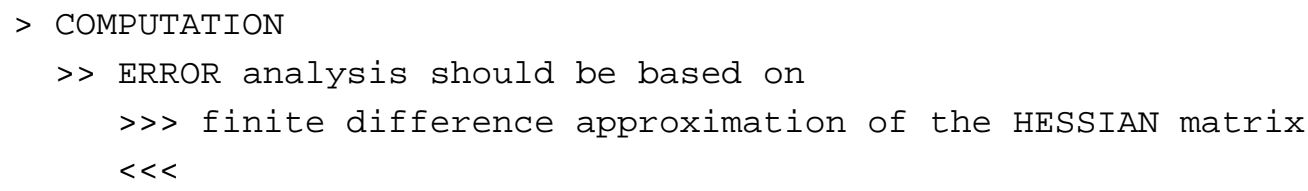

See Also

$>>$ LINEARITY 


\section{Command}

$>$ IFS

\section{Parent Command}

$>$ PARAMETER

\section{Subcommand}

$>>$ MODEL

\section{Description}

This command selects as a parameter one of the IFS parameters for describing heterogeneity (see Doughty [1995]). An index I must be provided through command $>>>>$ INDEX. If $I$ is positive, the parameter is one of the affine transform entries (variable PIFS(I)). If $I$ is negative, the parameter is the increment parameter for property field $-I$ (variable $\operatorname{TINC}(-I)$ ). If $I$ is lower than -100 , the parameter is the smoothing parameter in direction $(-I-100)$ (variable $\operatorname{SMOOTH}(-I-100)$ ).

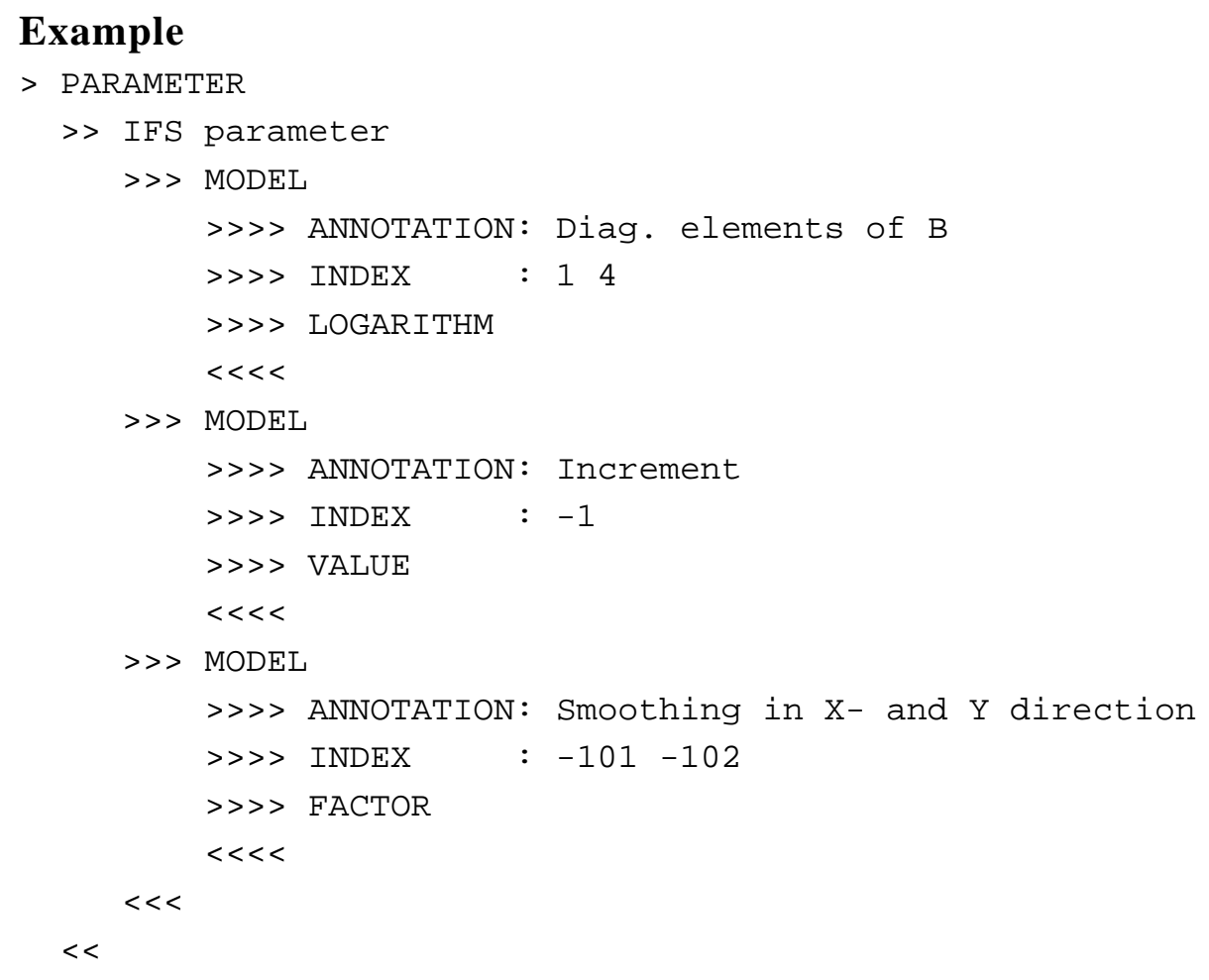

See Also

$-$ 


\section{Command}

>>> INCOMPLETE: max_incomplete

\section{Parent Command}

>> CONVERGE

\section{Subcommand}

$-$

\section{Description}

A successful iTOUGH2 run is based on the robustness and stability of the underlying TOUGH2 simulation. It is therefore imperative to develop a TOUGH2 model that is capable of completing the desired simulation for a variety of parameter combinations. The simulation must reach the time of the last calibration point, i.e., a premature termination due to convergence failures is not acceptable. The number of potential convergence failures or errors leading to premature termination is large. The type of convergence failure is indicated in the iTOUGH2 output file. It is usually impossible to continue the optimization process after a convergence failure. However, in some cases iTOUGH2 is able to retrieve information from a previous simulation which allows it to continue the inversion despite an incomplete run. iTOUGH2 always terminates if an incomplete run is encountered during the first evaluation of the Jacobian. The maximum number of incomplete simulations to be accepted by iTOUGH 2 can be set by variable max_incomplete (default: 5). However, one should not rely on inversions that contain incomplete TOUGH2 runs.

Note that if option $>>$ STEADY-STATE is invoked, all incomplete simulations are accepted by iTOUGH 2 assuming that steady-state conditions have been reached.

\section{Example}

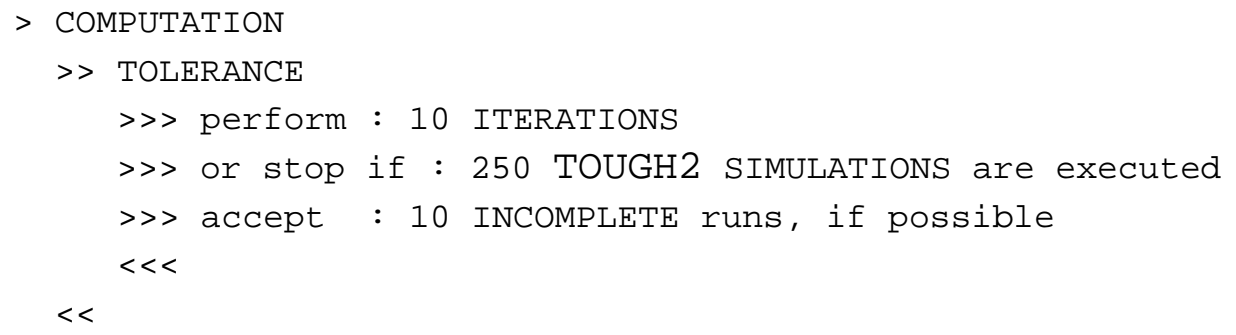

See Also

$>>$ STEADY-STATE 


\section{Command}

$>>$ INDEX

\section{Parent Command}

>> OUTPUT

\section{Subcommand}

\section{Description}

Prints the iTOUGH2 command index to the iTOUGH2 output file.

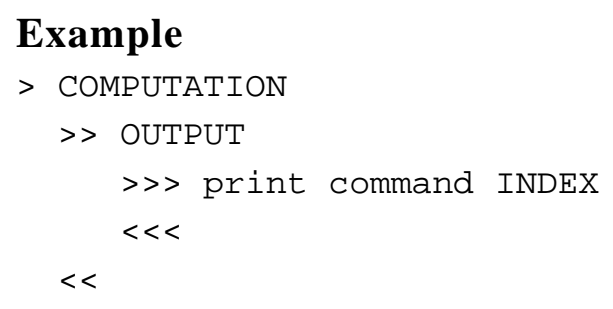

See Also

LIST, HELP 


\section{Command}

>>> INDEX: index (index_i...)

or

>>> PARAMETER: index (index_i...)

Parent Command

most third-level commands in block > PARAMETER

\section{Subcommand}

\section{Description}

This command provides a list of integers for further parameter specification. The integers are usually indexes of TOUGH2 arrays, such as IPAR in arrays CP(IPAR,NMAT) or $R P D(I P A R)$, selecting the IPAR-th parameter of the capillary pressure or default relative permeability function, respectively. If multiple indexes are provided, a single parameter will be estimated and assigned to all the corresponding array elements.

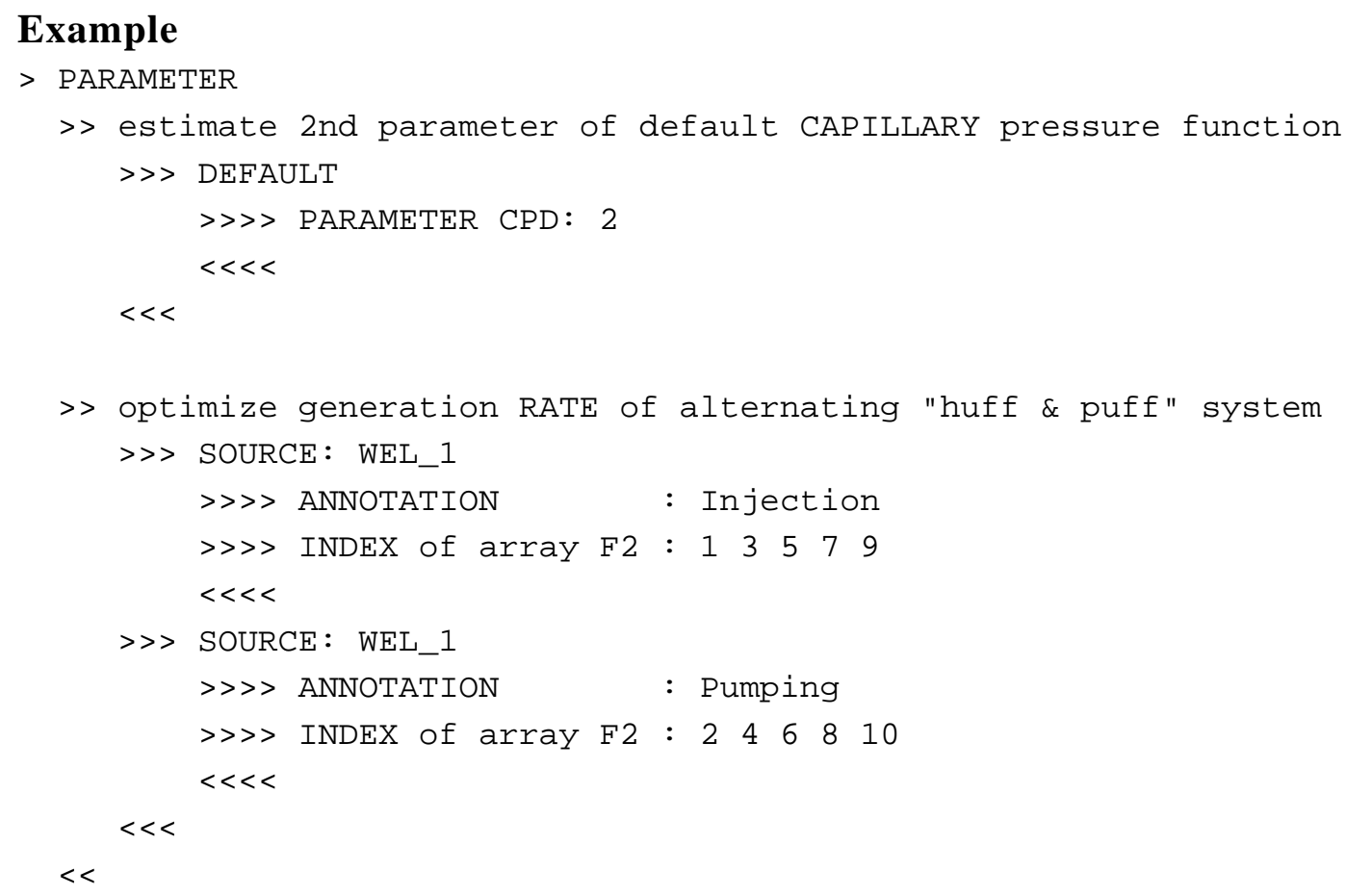

See Also

$-$ 


\section{Command}

>>> INDEX: index (index_i...)

or

>>> PARAMETER: index (index_i...)

Parent Command

all third-level commands in block > OBSERVATION

\section{Subcommand}

$-$

\section{Description}

This command provides a list of integers for further specification of user-specified observations (see command > USER (o)).

\section{Example}

(see command > USER (O))

See Also

>> USER (O) 


\section{Command}

>> INITIAL (PRESSURE/: ipv)

\section{Parent Command}

$>$ PARAMETER

\section{Subcommand}

$>>$ DEFAULT

$>>$ MATERIAL

\section{Description}

This command selects as a parameter the initial condition for all grid blocks associated with a certain rock type (TOUGH2 variable $D E P U(i p v)$ ) or default initial condition (TOUGH2 variable $D E P(i p v))$. Since boundary conditions are specified as initial conditions for inactive grid blocks or grid blocks with a large volume, this command can also be used to estimate boundary conditions. Initial conditions estimates cannot be provided for individual elements unless they have a unique material name associated with them. Estimating the first primary variable can be selected using keyword PRESSURE . All the other primary variables must be identified by number, i.e. by an integer ipv that follows a colon on the command line. Alternatively, ipv can be provided through command $>>>>$ INDEX. The initial guess for the parameter is taken from TOUGH2 block PARAM.4, or should be provided by using commands $>>$ GUESS or $>>>>$ GUESS or $>>>>$ PRIOR.

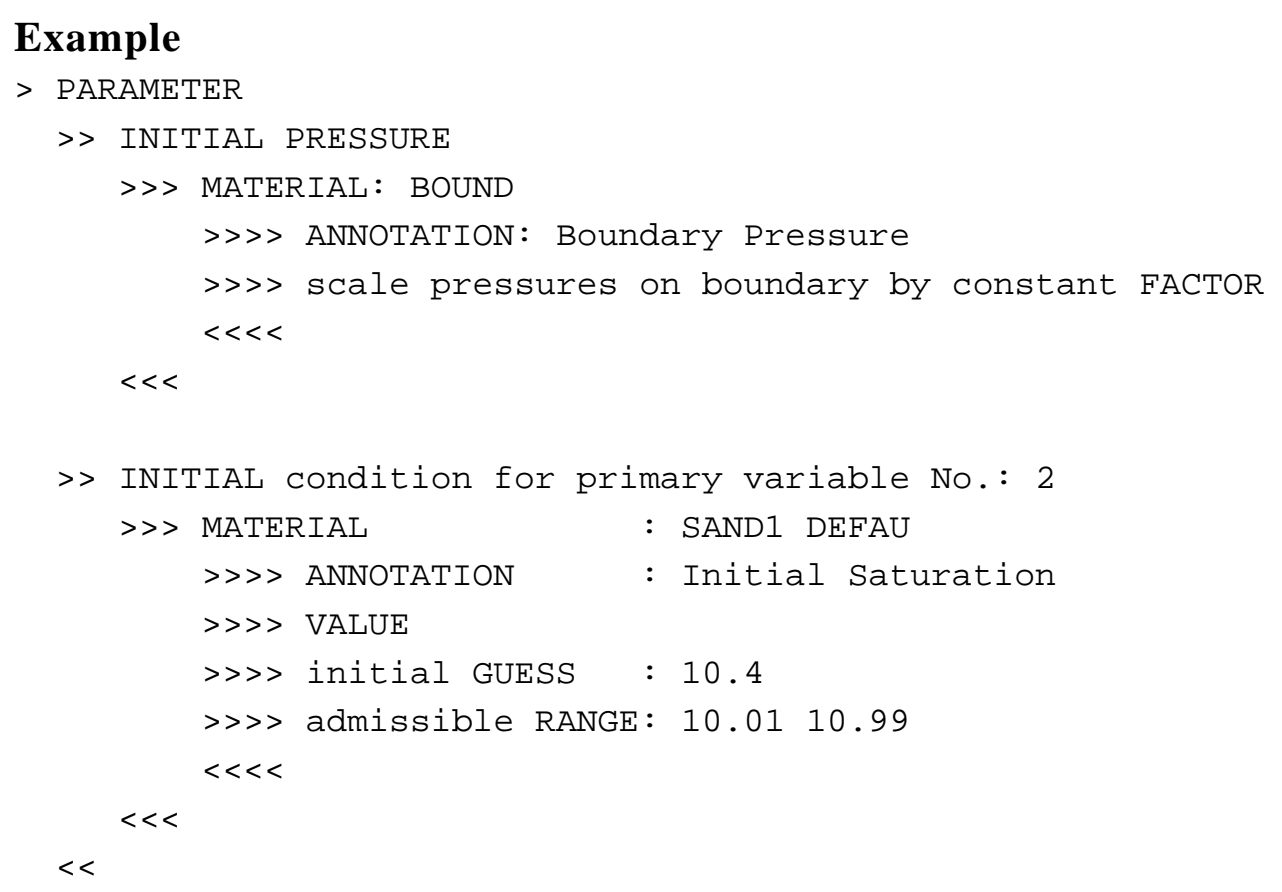

\section{See Also}




\section{Command}

$>>$ INPUT

\section{Parent Command}

>> CONVERGE

\section{Subcommand}

$-$

\section{Description}

This command makes iTOUGH2 stop immediately after the TOUGH2 and iTOUGH2 input files have been read and checked for consistency. This is useful to check input before time consuming simulations are invoked, or in combination with LIST, HELP, and $>>$ INDEX.

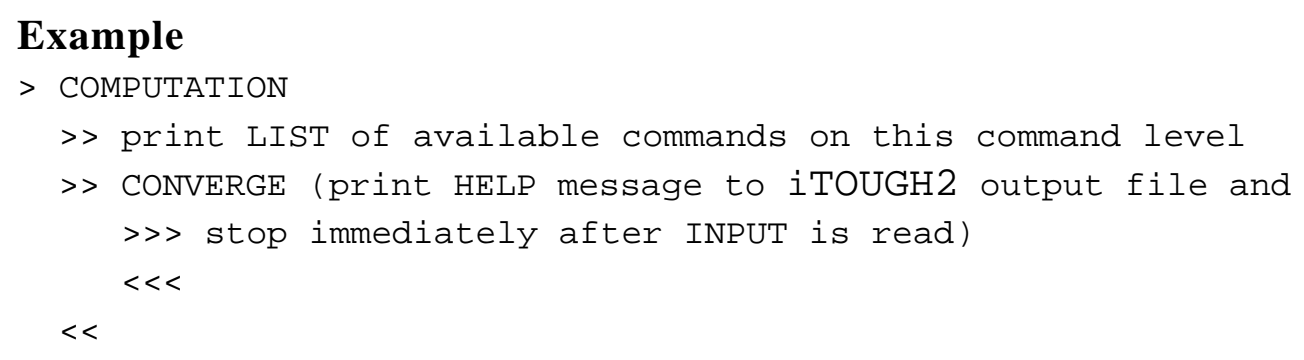

See Also

>> INDEX, HELP, LIST 


\section{Command}

>> INTERFACE: elem_l elem_2 (elem_i elem_j...) (+ iplus)

\section{Parent Command}

$>$ FLOW

Subcommand

all fourth-level commands in block > OBSERVATION

Description

(synonym for command >>> CONNECTION)

Example

(see command $>>>$ CONNECTION)

See Also

$>$ CONNECTION 


\section{Command}

>> ITERATION: max_iter

\section{Parent Command}

>> CONVERGE

\section{Subcommand}

\section{Description}

This command sets the maximum number of iTOUGH2 iterations to max_iter. If using the Levenberg-Marquardt minimization algorithm, an iTOUGH2 iteration consists of a number of TOUGH2 simulations and includes the following steps:

(1) solution of the forward problem,

(2) evaluation of the Jacobian matrix (requiring $n$ or $2 n$ TOUGH2 simulations depending on whether a forward or centered finite difference quotient is requested, see command >> JACOBIAN),

(3) updating of the parameter vector (see also command >>> STEP),

(4) check run(s) to see whether the new parameter set leads to a reduction of the objective function; if not, go back to step 3 .

If the objective function is successfully reduced, the iteration is completed, and the last check run is used as the solution of the forward problem (step (1) above) for the next iteration. By default, new iterations are performed until one of the following convergence criteria is met (note that different convergence criteria apply if options other than Levenberg-Marquardt optimization are used):

(1) the maximum number of TOUGH2 simulations is reached (see command >> SIMULATION),

(2) the maximum number of incomplete TOUGH2 simulations is reached (see command >> INCOMPLETE),

(3) the scaled step size is smaller than the minimum relative step size $=10^{-9}$,

(4) all parameters are at their user-specified bounds,

(5) the objective function is smaller than the relative function tolerance,

(6) the maximum number of unsuccessful uphill steps is exceeded (see command >> U UPHLL),

(7) the Levenberg parameter exceeds $10^{12}$,

(8) the norm of the gradient vector is smaller than $10^{-5}$ (optimality criterion).

In most cases, however, it is sufficient to stop the inversion after a few iterations because no significant fit improvement is obtained after about 5 to 15 iterations. Generally more iterations are required with increasing number of parameters and stronger non-linearities of the flow problem. 
The progress of the objective function reduction can be observed by typing the command prista (see unix script file prista), and inversion can be terminated using the $\mathrm{kit}$ command (see unix script file kit).

It is suggested to perform a single iTOUGH2 iteration or to use option $>>$ SENSITIVITY ANALYSIS prior to running a full inversion in order to check the relative importance and sensitivity of each parameter, the parameter step size, the initial value of the Levenberg parameter, etc.

\section{Example}

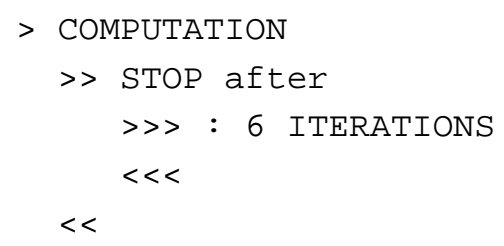

\section{See Also}

>> SENSITIVITY, >> CENTERED, >> FORWARD, >> INCOMPLETE, $>>$ SIMULATION, $>>>$ UPHILL 


\section{Command}

>>> ITERATION: max_iter

\section{Parent Command}

$>>$ ANNEAL

\section{Subcommand}

$-$

\section{Description}

This command limits the maximum number of iterations performed by the Simulated Annealing minimization algorithm. An iteration is completed if:

(1) the maximum number of steps $m_{\text {step }}$ on a temperature level is reached (see command $>>>\operatorname{STEP}$ (a)), or

(2) the objective function has been reduced $0.2 \cdot m_{\text {step }}$ times

Each iteration is followed by a reduction of the control parameter $\tau$ (temperature) according to the annealing schedule (see command >>> SCHEDULE).

\section{Example}

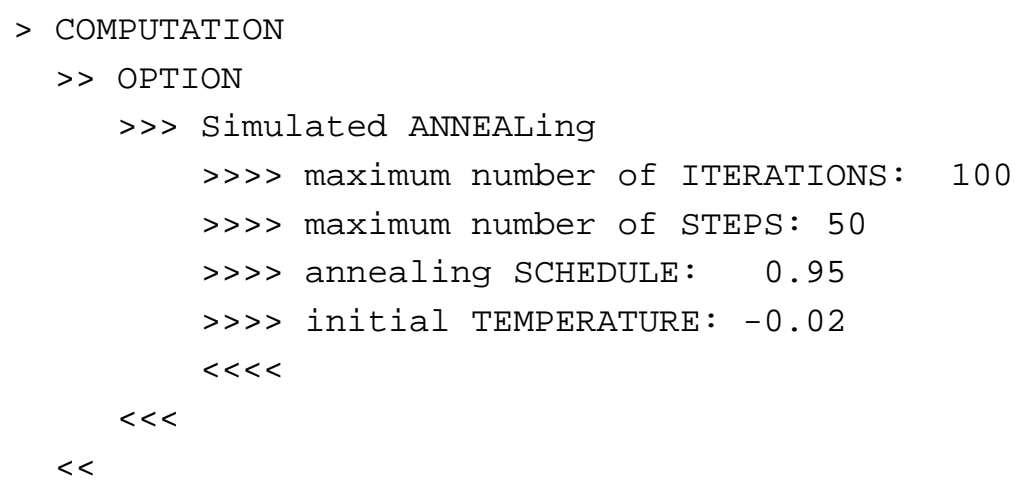

See Also

>>> STEP (a), >>> SCHEDULE 


\section{Command}

>>> ITERATION: niter

\section{Parent Command}

$>>$ SELECT

\section{Subcommand}

$-$

\section{Description}

This command defines the number of iterations after which the criteria for automatic parameter selection is reevaluated (see commands $>>>>$ CORRELATION and >>> SENSITIVITY).

A full Jacobian matrix is evaluated every multiple of niter iterations. In intermediate iterations, only the columns of the Jacobian corresponding to the selected parameters are updated.

\section{Example}

$>$ COMPUTATION

$>>$ OPTION

>> SELECT parameter automatically every

>>> : 3 ITERATIONS

$>>>$ based on the SENSITIVITY criterion with rsens : -0.10

$<<<<$

$<<<$

$<<$

See Also

>>> CORRELATION, >>> SENSITIVITY 


\section{Command}

>> JACOBIAN

Parent Command

$>$ COMPUTATION

\section{Subcommand}

$>>>$ CENTERED

$>>>$ FORWARD

$>>$ HESSIAN

$\gg>$ LIST

$>>$ PERTURB

\section{Description}

This is the parent command of a number of subcommands that deal with the calculation of the Jacobian matrix $\mathbf{J}$. The elements of the Jacobian matrix are the partial derivatives of the system response at the calibration points with respect to the parameters to be estimated:

$$
J_{i j}=-\frac{\partial r_{i}}{\partial p_{j}}=-\frac{\partial\left(z_{i}^{*}-z_{i}\right)}{\partial p_{j}}=\frac{\partial z_{i}}{\partial p_{j}}
$$

The Jacobian matrix discussed here must be distinguished from the one calculated in the simulation program TOUGH2. The latter is used to solve the set of non-linear algebraic iterations arising at each time step; its elements are the partial derivatives of the mass residuals with respect to the primary variables, and its numerical computation is controlled by the TOUGH2 variable DFAC.

\section{Example}

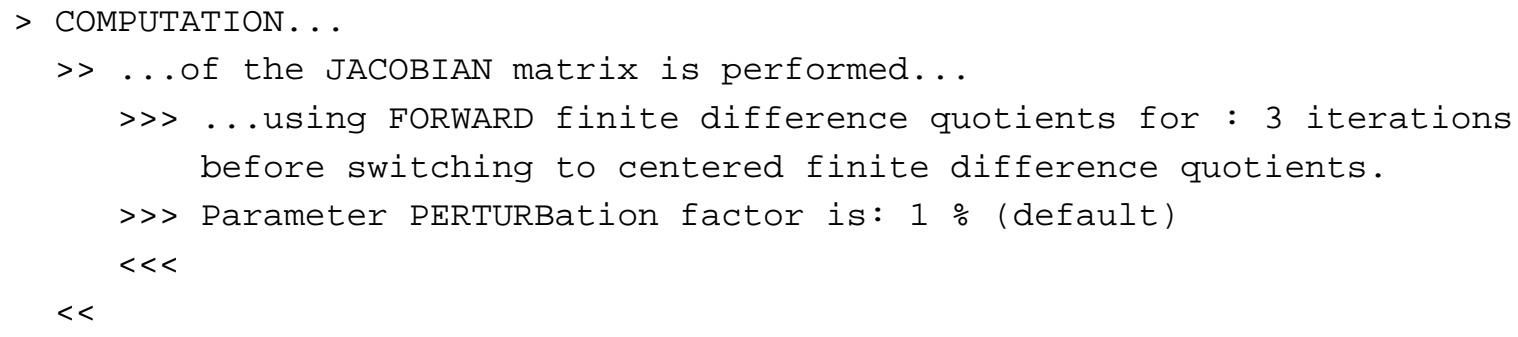

See Also 


\section{Command}

$>>$ JACOBIAN

\section{Parent Command}

$>$ OUTPUT

\section{Subcommand}

\section{Description}

This command prints the Jacobian matrix after each iteration. By default, the (scaled) Jacobian matrix is printed only once at the end of the optimization.

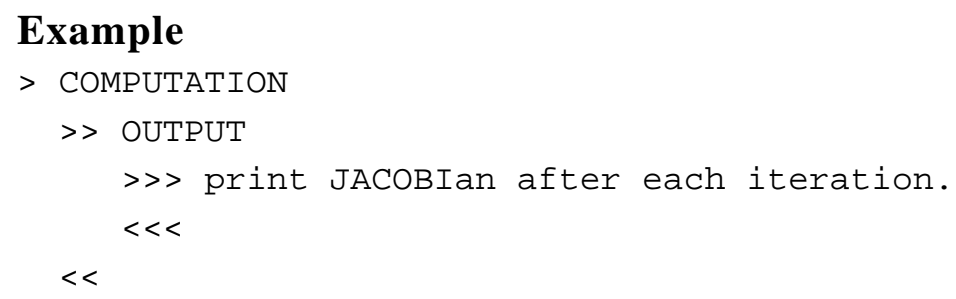

See Also

$>>$ RESIDUAL 


\section{Command}

>> KLINKENBERG

\section{Parent Command}

> PARAMETER

\section{Subcommand}

>> MATERIAL

\section{Description}

This command selects as a parameter the Klinkenberg slip factor (TOUGH2 variable GK (NMAT)).

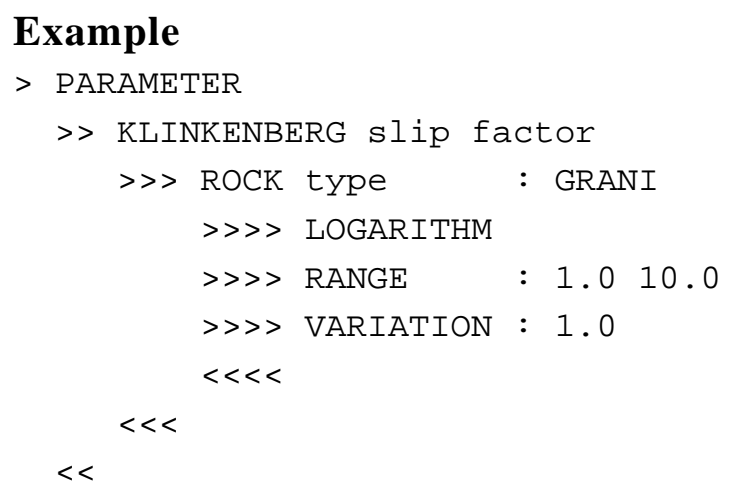

See Also 


\section{Command}

>> L1-ESTIMATOR

\section{Parent Command}

$>>$ OPTION

\section{Subcommand}

$-$

\section{Description}

This command selects the $\mathrm{L}_{1}$-estimator, i.e., the objective function to be minimized is the sum of the weighted absolute residuals.

$$
S=\sum_{i=1}^{m} \frac{\left|r_{i}\right|}{\sigma_{i}}
$$

Minimizing the mean absolute deviation leads to a maximum-likelihood estimate if the errors follow a double exponential distribution.

$$
\varphi\left(r_{i}\right)=\frac{1}{2 \sigma_{i}} \exp \left(-\left|\frac{r_{i}}{\sigma_{i}}\right|\right)
$$

The $\mathrm{L}_{1}$-estimator should be used, for example, to minimize a cost function for the optimization of a cleanup operation. Furthermore, it can be used whenever the objective function of interest is a linear function of the model output (e.g., in a sensitivity analysis using command $>>>$ OBJECTIVE in block $>>$ OPTION).

Note that this objective function is usually minimized using the Levenberg-Marquardt algorithm which is designed for a quadratic objective function. Minimization is therefore rather inefficient, requiring more iterations and a high initial Levenberg parameter.

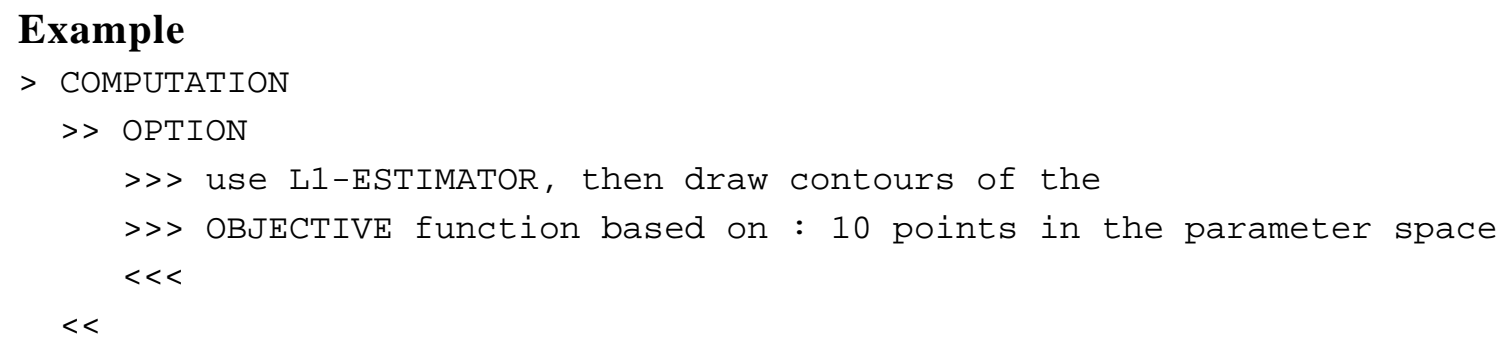

See Also

>> ANDREWS, >>> CAUCHY, >>> LEAST-SQUARES,

$>>$ QUADRATIC-LINEAR 


\section{Command}

$>$ LAG

\section{Parent Command}

$>$ PARAMETER

\section{Subcommand}

$>>$ NONE

$>>\mathrm{SET}$

\section{Description}

This command selects as a parameter a constant lag, shifting data in time. The time lag is applied to the output that refers to a specific data set:

$$
z(t)=z_{\text {TOUGH2 }}(t+l a g)
$$

Here, $t$ is time, lag is the estimated time lag in seconds, and $z_{\text {TOUGH2 } 2}$ is the TOUGH2 output. The result $z$ is compared to the measurement $z^{*}$ of the corresponding data set. A single data set is identified by number using command $>>>\operatorname{SET}(\mathrm{p})$; multiple data sets are specified with command $>>>>\operatorname{INDEX}(p)$.

If the lag is known, i.e., does not need to be estimated, use command $>>>>$ SHIFT TIME, a subcommand of > OBSERVATION.

\section{Example}

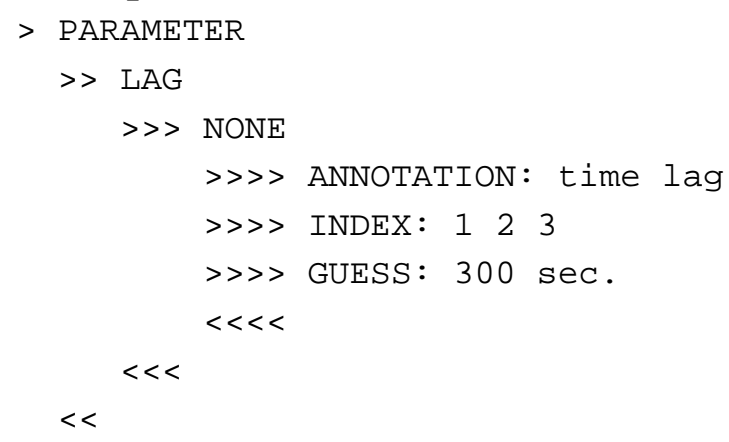

See Also

$>>\operatorname{DRIFT},>>\operatorname{FACTOR},>>\operatorname{SHIFT},>>\operatorname{SET}(\mathrm{p}),>>>>\operatorname{INDEX}(\mathrm{p})$, $>>>$ SHIFT 


\section{Command}

>> LEAST-SQUARES

\section{Parent Command}

$>$ OPTION

\section{Subcommand}

$-$

\section{Description}

This command selects least-squares optimization, i.e., the objective function to be minimized is the sum of the squared weighted residuals.

$$
S=\mathbf{r}^{T} \mathbf{C}_{z z}^{-1} \mathbf{r}=\sum_{i=1}^{m}\left(\frac{r_{i}}{\sigma_{i}}\right)^{2}
$$

Minimizing the squared weighted residuals leads to a maximum-likelihood estimate if the errors are normally distributed with zero mean and covariance matrix $\mathbf{C}_{z z}$ :

$$
\varphi(\mathbf{r})=(2 \pi)^{-m / 2}\left|\mathbf{C}_{z z}\right|^{-1 / 2} \exp \left(-\frac{1}{2} \mathbf{r}^{T} \mathbf{C}_{z z}^{-1} \mathbf{r}\right)
$$

Least-squares estimation is the default. If outliers are more prominent than described by the tail of the normal distribution, one may want to use one of the robust estimators to reduce the weight of outliers or even eliminate them (see commands $>>>$ ANDREWS, >> CAUCHY, or $>>>$ QUADRATIC-LINEAR).

The least-squares objective function is a quadratic function if the residuals depend linearly on the parameters; it is nearly-quadratic for a non-linear model. The Levenberg-Marquardt algorithm is best suited to minimize the non-linear least-squares objective function.

\section{Example}

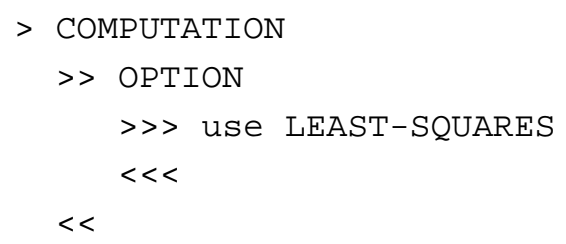

\section{See Also}

>> ANDREWS, >>> CAUCHY, >>> L1-ESTIMATOR, >>> QUADRATIC-LINEAR 


\section{Command}

>> LEVENBERG: lambda

\section{Parent Command}

>> CONVERGE

\section{Subcommand}

$-$

\section{Description}

This command sets the initial value of the Levenberg parameter $\lambda$ (default: 0.001 ). During the optimization process (see command $>>>$ LEVENBERG-MARQUARDT), the Levenberg parameter is divided by the Marquardt parameter $v$ (see command $>>>$ MARQUARDT) after each successful iteration, and is multiplied by $v$ if the new parameter set leads to an increased value of the objective function, i.e., if an unsuccessful step was proposed. A big value for $\lambda$ means that a small step along the steepest descent direction is performed. A $\lambda$ value of zero is equivalent to a Gauss-Newton step. The former is robust but inefficient, the latter has a quadratic convergence rate but may lead to unsuccessful steps.

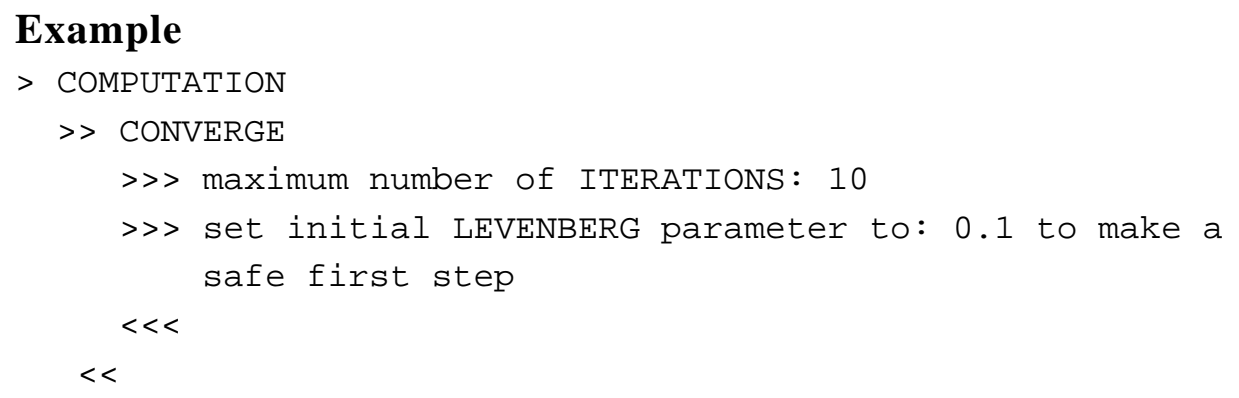

See Also

>> MARQUARDT 


\section{Command}

>> LEVENBERG-MARQUARDT

\section{Parent Command}

>> OPTION

\section{Subcommand}

$-$

\section{Description}

This command selects the Levenberg-Marquardt algorithm to minimize the objective function. This is the default minimization algorithm. The Levenberg-Marquardt algorithm combines the robustness of a steepest descent method with the efficiency of a Gauss-Newton step (see command >>> GAUSS-NEWTON):

$$
\begin{gathered}
\Delta \mathbf{p}=\left(\mathbf{J}^{T} \mathbf{C}_{z z}^{-1} \mathbf{J}+\lambda \mathbf{D}\right)^{-1} \mathbf{J}^{T} \mathbf{C}_{z z}^{-1} \mathbf{r} \\
\mathbf{p}^{(k+1)}=\mathbf{p}^{(k)}+\Delta \mathbf{p}
\end{gathered}
$$

where $\mathbf{D}$ is a diagonal matrix with elements $D_{i i}=\left(\mathbf{J}^{T} \mathbf{C}_{z z}^{-1} \mathbf{J}\right)_{i i}$.

The Levenberg-Marquardt method switches continuously from a gradient method (large $\lambda$, see command >> LEVENBERG) far from the minimum to a Gauss-Newton step as the minimum is approached and $\lambda$ is reduced.

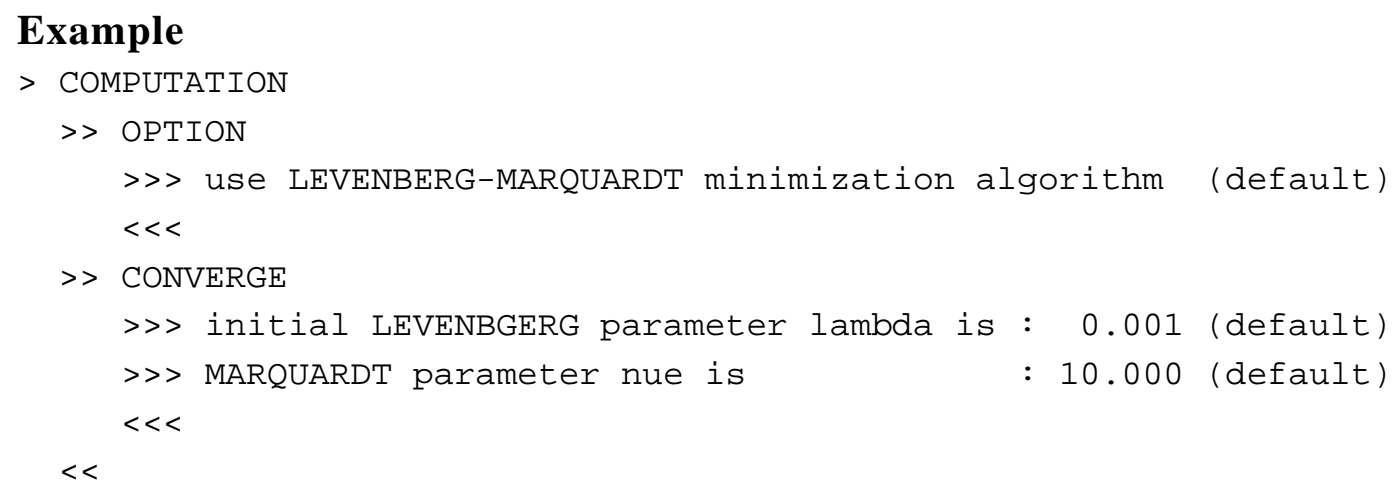

See Also

>>> ANNEAL, >>> GAUSS-NEWTON, >>> GRID SEARCH, >>>SIMPLEX,

$>>>$ LEVENBERG, $>>>$ MARQUARDT 


\section{Command}

>> LINEARITY (: alpha (\%))

Parent Command

>> ERROR

\section{Subcommand}

\section{Description}

The covariance matrix of the estimated parameters, $\mathbf{C}_{p p}$, is calculated using linear error analysis:

$$
\mathbf{C}_{p p}=s_{0}^{2}\left(\mathbf{J}^{T} \mathbf{C}_{z z}^{-1} \mathbf{J}\right)^{-1}
$$

The confidence region around the estimated parameter set for the linearized case consists of those values $\mathbf{p}$ for which

$$
(\mathbf{p}-\hat{\mathbf{p}})^{T} \mathbf{C}_{p p}(\mathbf{p}-\hat{\mathbf{p}}) \leq n \cdot F_{n, m-n, 1-\alpha}
$$

where $\hat{\mathbf{p}}$ is the parameter vector at the optimum. The covariance matrix $\mathbf{C}_{p p}$ approximates the actual surface of the objective function at its minimum by a tangent hyperellipsoid under the assumption of normality and linearity. If the model is nonlinear, the coverage of the confidence region by the linear approximation may be very poor with respect to both its size and its shape.

When using this command, it is assumed that the shape of the confidence region is close to ellipsoidal, and that the orientation of the hyperellipsoid in the $n$-dimensional parameter space is accurately obtained from the linear error analysis. Then, by only adjusting the size of the hyperellipsoid, we can better approximate the confidence region without losing the advantage of producing easily understandable results which are also simple to report. The procedure adopted here is based on a comparison of the actual objective function with the results from the linear approximation at discrete points in the parameter space. These test points $\tilde{\mathbf{p}}$ are located along the main axis of the hyperellipsoid, i.e.:

$$
\tilde{\mathbf{p}}_{i \pm}=\hat{\mathbf{p}} \pm\left(n \cdot F_{n, m-n, 1-\alpha}\right)^{1 / 2} a_{i} \mathbf{u}_{i} \quad i=1, \cdots, n
$$

Here, $\tilde{\mathbf{p}}_{i \pm}$ are two test parameter sets on the $i$-th axis, the direction of which is given by the eigenvector $\mathbf{u}_{i}$ of the covariance matrix $\mathbf{C}_{p p}$. The distance from the optimal parameter set $\hat{\mathbf{p}}$ is selected as a multiple of the corresponding eigenvalue $a_{i}^{2}$ and the quantile of the $F$ distribution. This means that the correction is tailored to approximate the confidence region on a certain confidence level $1-\alpha$. The eigenvalues $a_{i}^{2}$, which determine the length of the semiaxis, are now corrected as follows: 


$$
a_{i}^{\prime 2}=a_{i}^{2} s_{0}^{2}\left(\frac{A_{+}+A_{-}}{2}\right)_{i}
$$

with

$$
A_{ \pm i}=\frac{n \cdot F_{n, m-n, 1-\alpha}}{S\left(\tilde{\mathbf{p}}_{i \pm}\right)-S(\hat{\mathbf{p}})}
$$

Finally, the new covariance matrix is back-calculated from the eigenvectors $\mathbf{u}_{i}$ and the updated eigenvalues $a_{i}^{\prime 2}$.

This correction procedure requires $2 n$ additional solutions of the direct problem and is thus relatively inexpensive. While the resulting confidence region is ellipsoidal by definition, the differences between $S\left(\tilde{\mathbf{p}}_{i+}\right)$ and $S\left(\tilde{\mathbf{p}}_{i-}\right)$ provide, as a byproduct of the correction procedure, some insight into the asymmetry of the true confidence region.

The user may specify $\alpha$ or $1-\alpha$ in $\%$ or the quantile $\left(n \cdot F_{n, m-n, 1-\alpha}\right)^{1 / 2}$ directly (by omitting $\div$ on the command line).

Note that the correction procedure fails if the minimum is not accurately identified. In this case iTOUGH2 automatically proceeds with the covariance matrix from the linear error analysis.

\section{Example}

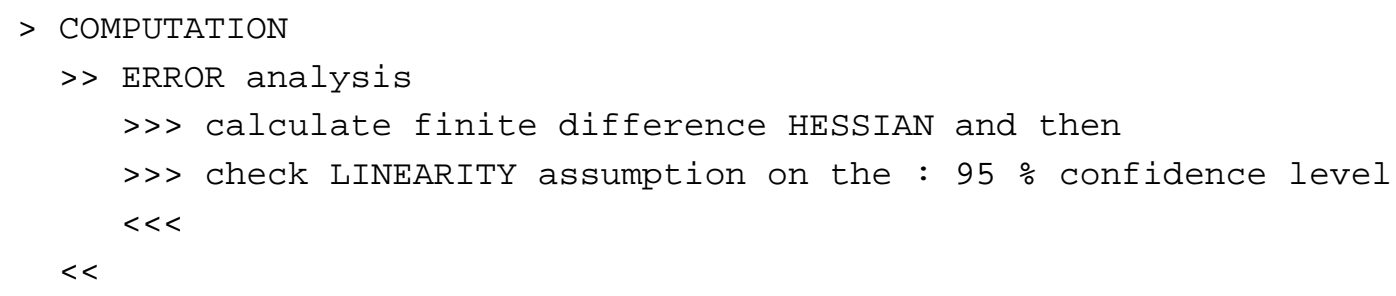

See Also

$>>$ HESSIAN 


\section{Command}

LIST (available on all command levels)

\section{Parent Command}

\section{Subcommand}

$-$

\section{Description}

This command provides a list of all available commands on the corresponding command level. The list contains the actual upper case spelling of the command as interpreted by the program. Potential keywords are not listed. A complete list of all commands can be obtained with command $>>$ command INDEX.

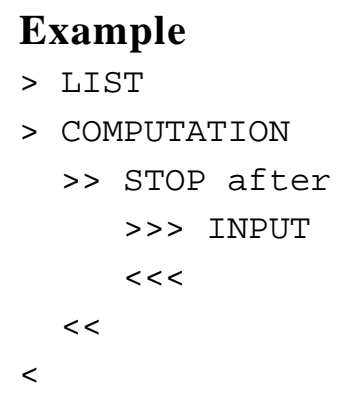

The following list is printed to the iTOUGH2 output file showing all first-level commands:

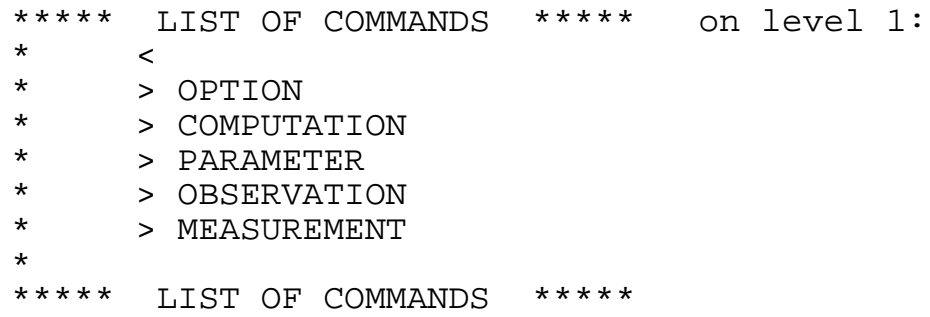

See Also

$>>$ INDEX, >> INPUT 


\section{Command}

>>> LOGARITHM

Parent Command

all third-level commands in block > PARAMETER

\section{Subcommand}

\section{Description}

The parameter $p$ to be estimated is the logarithm of the TOUGH2 parameter $X$ :

$$
p=\log _{10}(X) \Leftrightarrow X=10^{p}
$$

Estimation of logarithms is recommended if the parameter is expected to vary over a large range of values, suggesting a log-normal distribution of its estimate. Note that all quantities referring to this parameter are also in log-space (e.g., range, standard deviation, step length, etc.). Estimating the logarithm is chosen her eover estimating the parameter value directly (command $>>>>$ VALUE), estimating a multiplication factor (command $>>>>$ FACTOR (p) ), or estimating a log-normally distributed multiplication factor (command $>>>$ LOG $(\mathrm{F})$.

\section{Example}

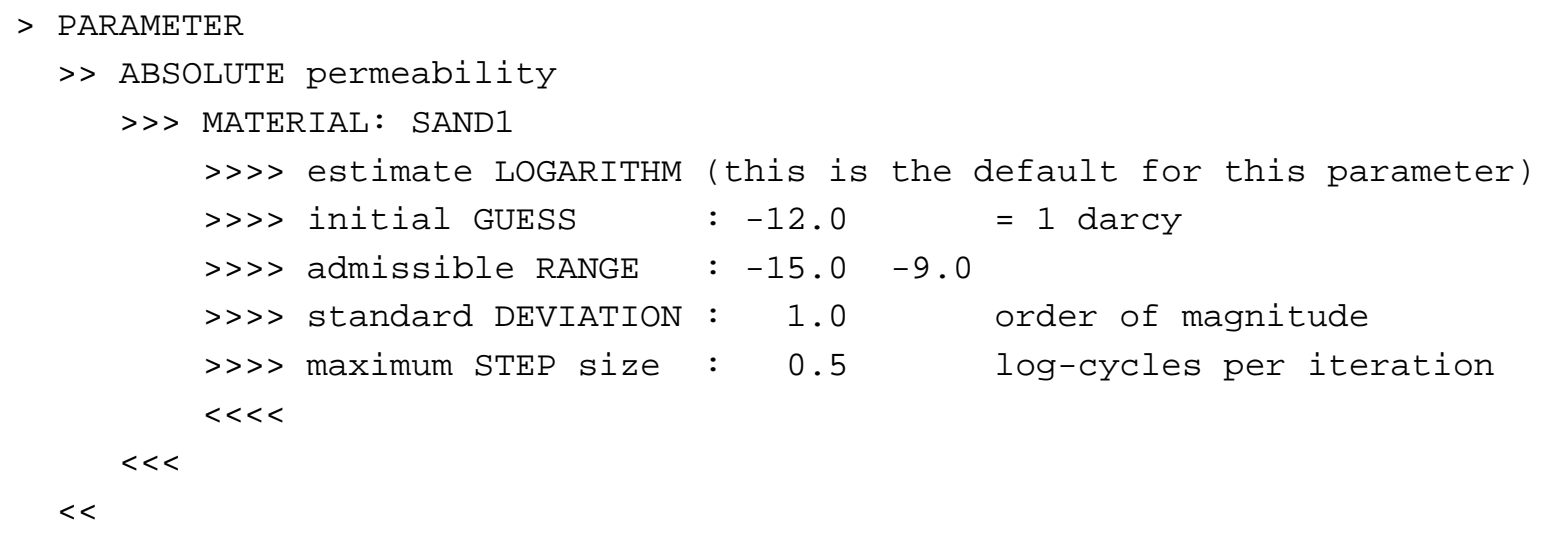

See Also

$>>>>$ FACTOR $(p),>>>>$ LOG $(F), \quad>>>>$ VALUE 


\section{Command}

>>> LOGARITHM

Parent Command

all third-level commands in block > OBSERVATION

\section{Subcommand}

$-$

\section{Description}

This command takes the logarithm (base 10) of an observable variable. The corresponding measurement error is assumed to be log-normally distributed. Taking the logarithm is suggested when the observation assumes values over many orders of magnitude (e.g., concentration, water potential). Note that this option emphasizes the importance of smaller values of the variable, and is not applicable to data sets that contain zero measurements.

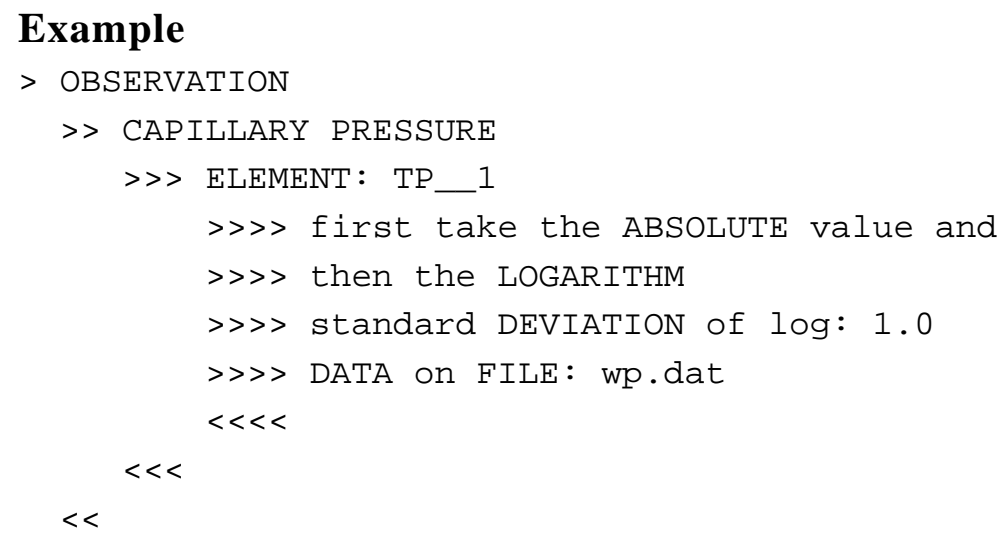

See Also 


\section{Command}

$>>>$ LOG $(F)$

\section{Parent Command}

all third-level commands in block > PARAMETER

\section{Subcommand}

\section{Description}

The parameter to be estimated is a log-normally distributed factor with which the initial TOUGH2 parameter value is multiplied:

$$
p=\log _{10}\left(X / X_{0}\right) \Leftrightarrow X=10^{p} \cdot X_{0}
$$

Here, $p$ is the estimated parameter, $X$ is the TOUGH2 parameter, and $X_{0}$ is the initial value of the TOUGH2 parameter. This option is useful to determine the mean of a log-normally distributed quantity while maintaining ratios (e.g., if estimating a common factor applied to all three permeability values in a model domain, the anisotropy ratio remains constant). Estimating a factor is chosen here over estimating the parameter value directly (command >>> VALUE) or its logarithm (command >>> LOGARITHM (p)).

\section{Example}

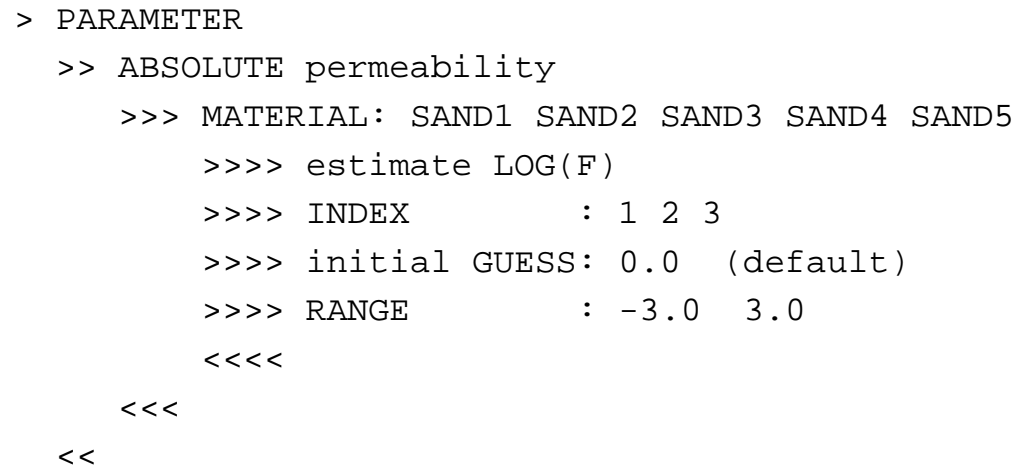

See Also

$>>>$ LOGARITHM $(\mathrm{p}),>>>$ FACTOR, $>>>>$ VALUE 


\section{Command}

>> MARQUARDT: nue

\section{Parent Command}

>> CONVERGE

\section{Subcommand}

$-$

\section{Description}

This command sets the Marquardt parameter $v$ (default: 10.0). During the optimization process, the Levenberg parameter $\lambda$ (see command $>>>$ LEVENBERG) will be divided by the Marquardt parameter $v$ after each successful iteration, and it will be multiplied by $v$ if the new parameter set leads to an increased value of the objective function, i.e., if an unsuccessful step was proposed.

A big value for $\lambda$ means that a small step along the gradient direction is performed. A $\lambda$ value of zero is equivalent to a Gauss-Newton step. The former is robust but inefficient, the latter has a quadratic convergence rate but may lead to unsuccessful steps.

The Marquardt parameter therefore determines how fast the step size and step direction changes from steepest descent to Gauss-Newton and vice versa.

\section{Example}

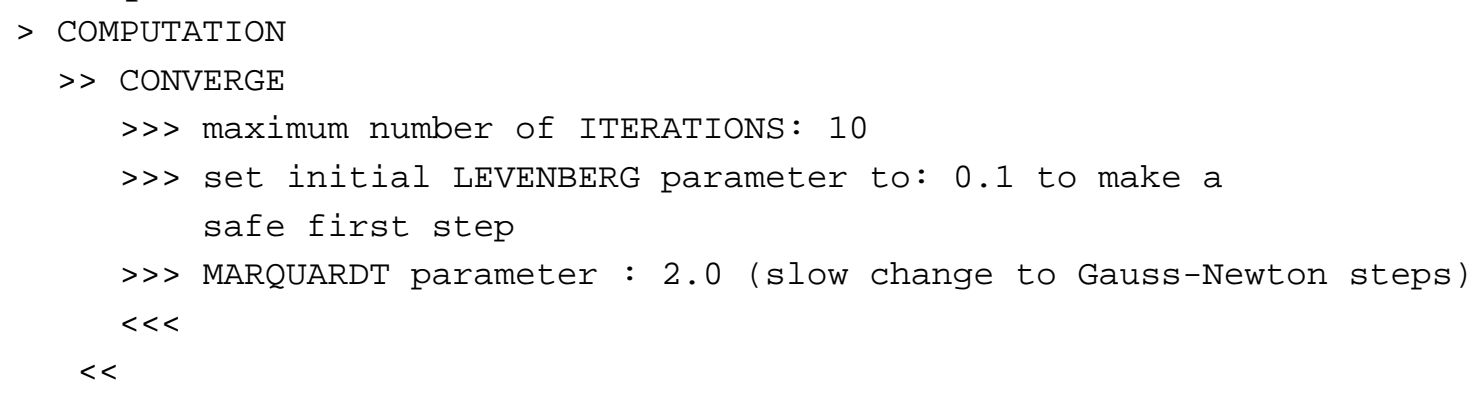

See Also

>>> LEVENBERG 


\section{Command}

>> MASS FRACTION (comp_name/COMPONENT: icomp)

(phase_name/PHASE: iphase)

Parent Command

$>$ OBSERVATION

\section{Subcommand}

$>>$ ELEMENT

\section{Description}

This command selects as an observation type the mass fraction of component icomp in phase iphase. This observation type refers to one or more elements.

Component number icomp or component name comp_name, and phase number iphase or phase name phase_name depend on the EOS module being used. They are listed in the iTOUGH2 header, and can be specified either on the command line or using the two subcommands >>> COMPONENT and >>> PHASE, respectively.

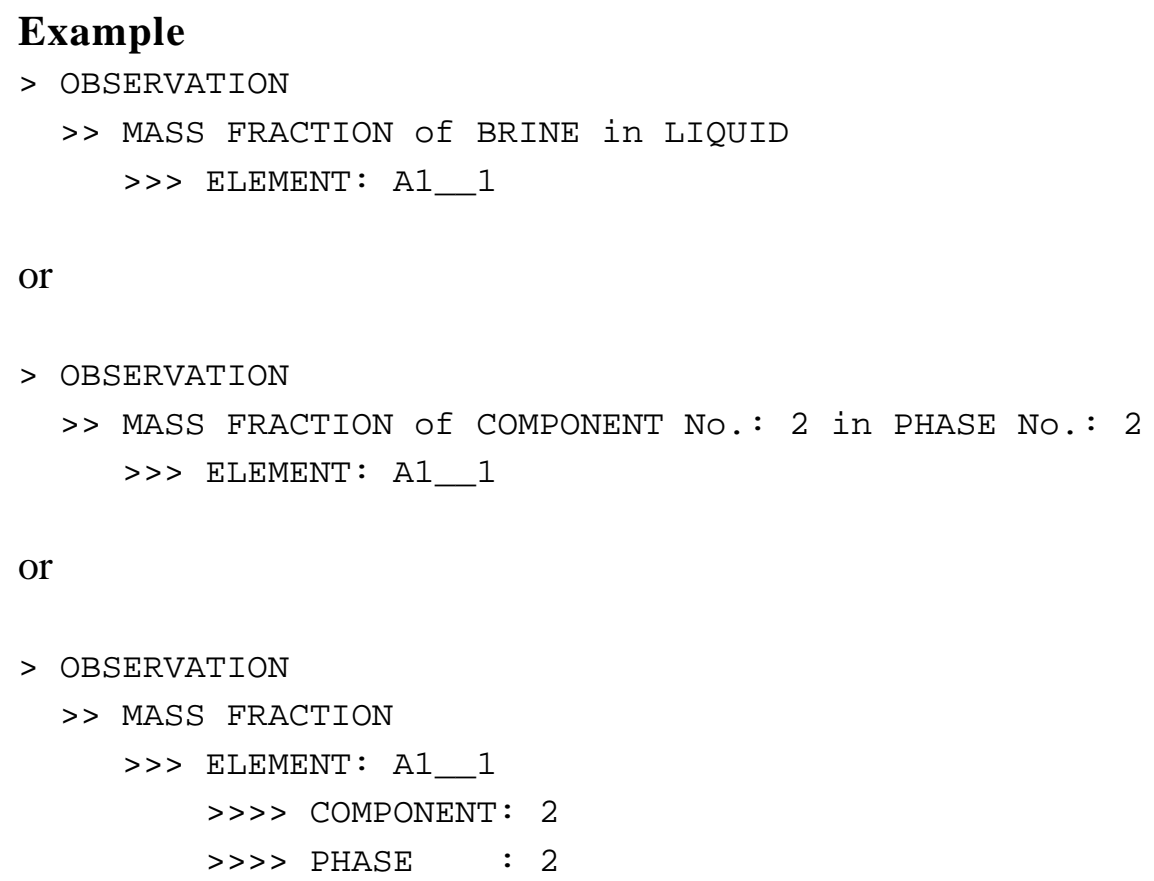

See Also

$>$ CONCENTRATION 


\section{Command}

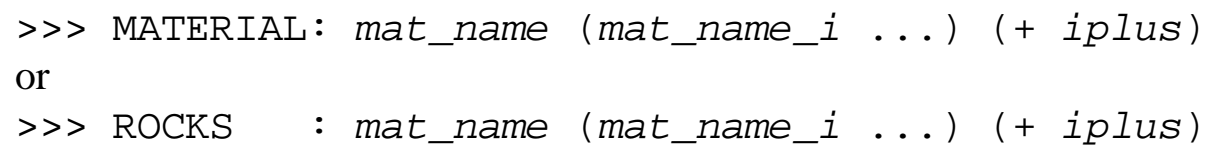

\section{Parent Command}

all second-level commands in block > PARAMETER referring to a material name

\section{Subcommand}

all fourth-level commands in block > PARAMETER

\section{Description}

This command identifies material names (TOUGH2 variable MAT). Most parameters refer to a particular material, i.e., they are specified in TOUGH2 in block ROCKS. Rock types are designated by a five-character code name. Blanks in the material name must be replaced by underscores (e.g., if MAT in TOUGH2 reads 'CLAY ', mat_name must read 'CLAY_'). If multiple material names are provided, the estimate of the corresponding parameter will be jointly assigned to all listed materials. If distinct parameters are sought for each rock type, separate >>> MATERIAL blocks must be defined. Default properties (i.e., for parameters referring to TOUGH2 blocks PARAM.4 and RPCAP) can be selected either by >> DEFAULT Or >> MATERIAL: DEFAU.

If the last two characters of the last mat_name are integers, a sequence of iplus material names can be generated. The following two command lines are thus identical:

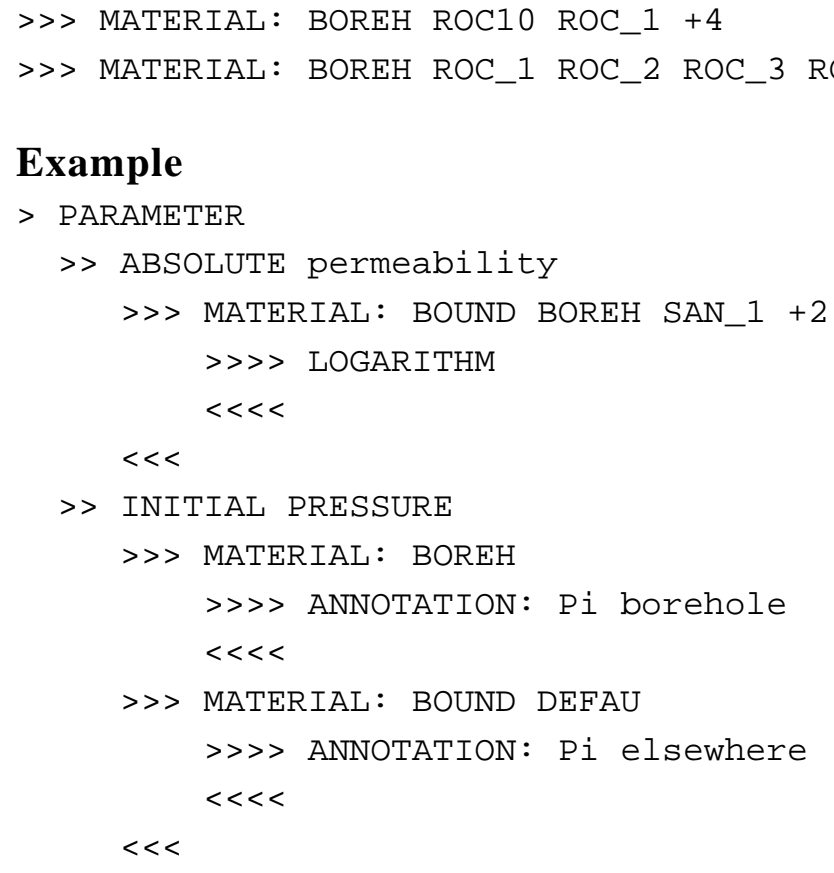

See Also

$>>$ DEFAULT, $>>$ MODEL 


\section{Command}

>>> MEAN (VOLUME)

\section{Parent Command}

all third-level commands in block > OBSERVATION

\section{Subcommand}

$-$

\section{Description}

(synonym for command $>>>$ AVERAGE)

\section{Example}

(see command $>>>>$ AVERAGE)

\section{See Also}

>>> AVERAGE 


\section{Command}

$>$ MINC

\section{Parent Command}

$>$ PARAMETER

\section{Subcommand}

$>>$ MODEL

\section{Description}

This command selects as a parameter the fracture spacing which is a parameter of the MINC preprocessor (TOUGH2 variable $P A R M I N C(I)$ ). The fracture set is identified through command $>>>>$ INDEX. This parameter refers to the entire model.

Estimating fracture spacing is only possible if the mesh can be generated internally without further editing by the user. Single elements and connections can be modified, however, by means of TOUGH2 commands ELEM2 and CONN2, respectively. For example, if a mesh is generated using MESHMAKER and MINC, the volumes of individual boundary elements can be adjusted in the ELEM2 block, i.e., avoiding the need for external editing.

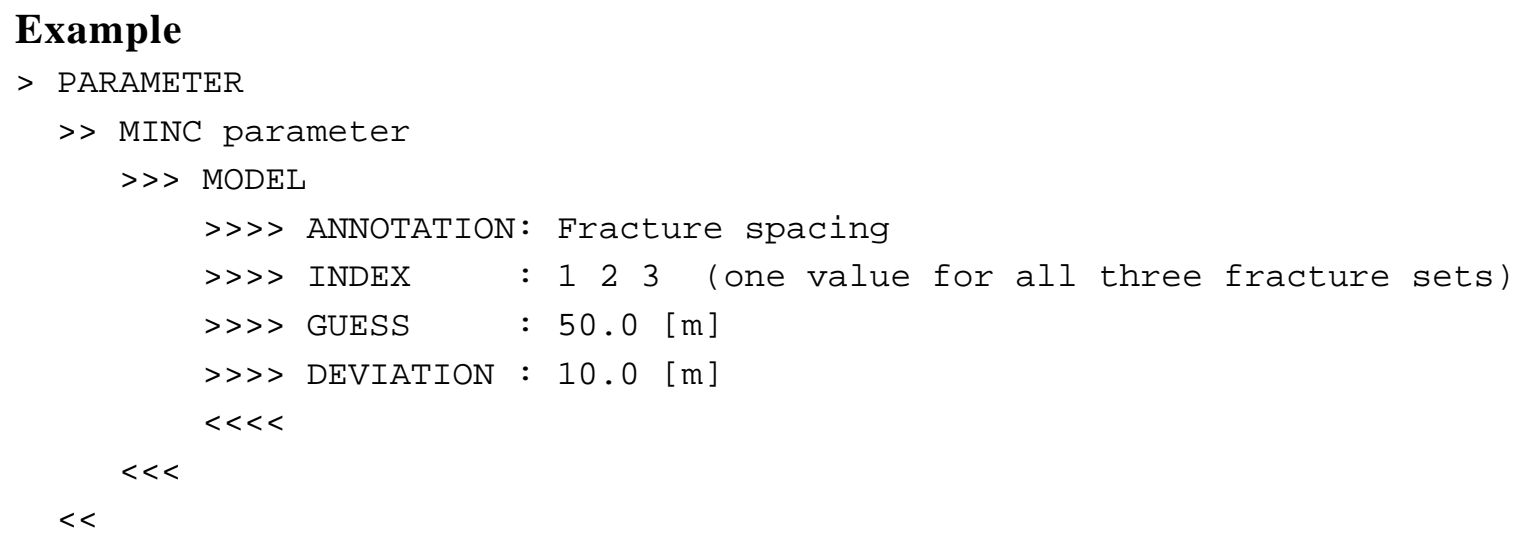

See Also 


\section{Command}

$>>$ MODEL

or

$>>$ NONE

\section{Parent Command}

all second-level commands in block > PARAMETER or > OBSERVATION addressing parameters or observations that refer to the entire model domain or a non-specific item.

\section{Subcommand}

all fourth-level commands in block > PARAMETER or > OBSERVATION, respectively.

\section{Description}

Most parameters refer to a rock type or code name of a sink or source. Some parameters, however, are general or associated with the entire model domain. Similarly, while most observations refer to an element, connection, or sink/source, there are observation types that are not associated with a well-defined point in space. The commands >>> MODEL or >> NONE are dummy commands, i.e. place holders on the third command level for parameter and observation types not further specified by one of the other third-level commands.

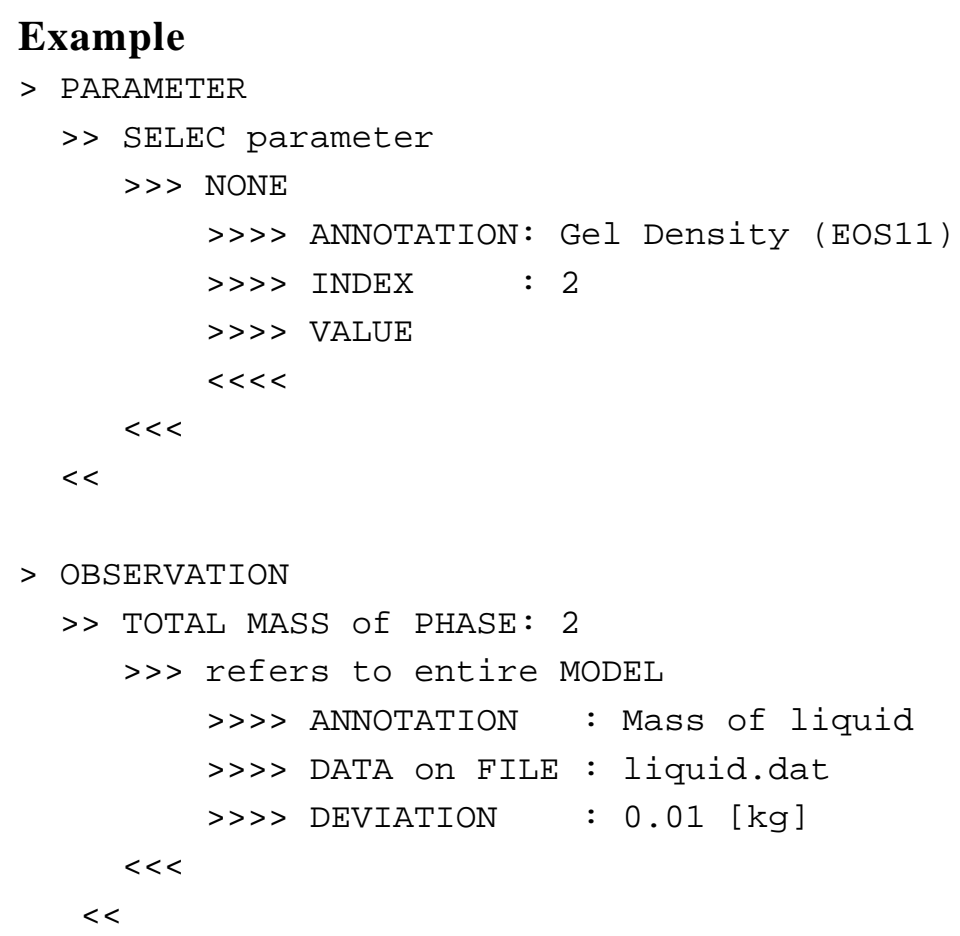




\section{Command}

>> MOMENT (FIRST/SECOND) (X/Y/Z) (comp_name/COMPONENT: icomp) (phase_name/PHASE: iphase)

\section{Parent Command}

$>$ OBSERVATION

\section{Subcommand}

$>>$ MODEL

\section{Description}

This command selects as an observation type the first or second spatial moment in $\mathrm{X}, \mathrm{Y}$, or Z direction of component icomp or phase iphase. This observation type refers to all elements with $10,000>S P H T>0$. The $\mathrm{X}, \mathrm{Y}$, and $\mathrm{Z}$ coordinates of the grid blocks must be given in TOUGH2 block ELEME, columns 51-60, 61-70, 71-80, respectively.

Component number icomp or component name comp_name, and phase number iphase or phase name phase_name depend on the EOS module being used. They are listed in the iTOUGH2 header, and can be specified either on the command line or using the two subcommands >>> COMPONENT and >>> PHASE, respectively. If only a phase but no component is specified, the spatial moment of the indicated phase including all components is calculated. If only a component but no phase is given, the spatial moment of that component in all phases is calculated. If both a component and a phase are given, the spatial moment of the component in the specified phase is calculated.

The spatial moments are calculated as follows:

$$
M_{i j k}=\sum_{\beta} \sum_{\kappa} \sum_{n=1}^{N E L}\left(X^{i} \cdot Y^{j} \cdot Z^{k} \cdot V \cdot \phi \cdot S_{\beta} \cdot \rho_{\beta} \cdot X_{\beta}^{\kappa}\right)_{n}
$$

where the first sum is taken over the selected phase(s) $\beta$, the second sum is taken over the selected component(s) $\kappa$, and the third summation accumulates the masses of component $\kappa$ in phase $\beta$ from all elements that are included in the global mass balance calculation (i.e., elements with a rock grain specific heat lower than $10^{4} \mathrm{~J} / \mathrm{kg}^{\circ} \mathrm{C}$ ). The mass in element $n$ is the product of the element volume $V_{n}$, the porosity $\phi_{n}$, the saturation $S_{\beta n}$, the phase density $\rho_{\beta n}$, and the mass fraction $X_{\beta n}^{\kappa}$.

The first moment represents the center of mass coordinates which are given by

$$
\begin{aligned}
& \langle X\rangle=M_{100} / M_{000} \\
& \langle Y\rangle=M_{010} / M_{000} \\
& \langle Z\rangle=M_{001} / M_{000}
\end{aligned}
$$

The second moment represents the variances in the three directions given by 


$$
\begin{aligned}
\sigma_{X}^{2} & =\frac{M_{200}}{M_{000}}-\langle X\rangle^{2} \\
\sigma_{Y}^{2} & =\frac{M_{020}}{M_{000}}-\langle Y\rangle^{2} \\
\sigma_{Z}^{2} & =\frac{M_{002}}{M_{000}}-\langle Z\rangle^{2}
\end{aligned}
$$

This option may be especially useful for characterizing the location and spreading of a contaminant or saturation plume. Note that boundary effects may have a strong impact on spatial moment calculation.

\section{Example}

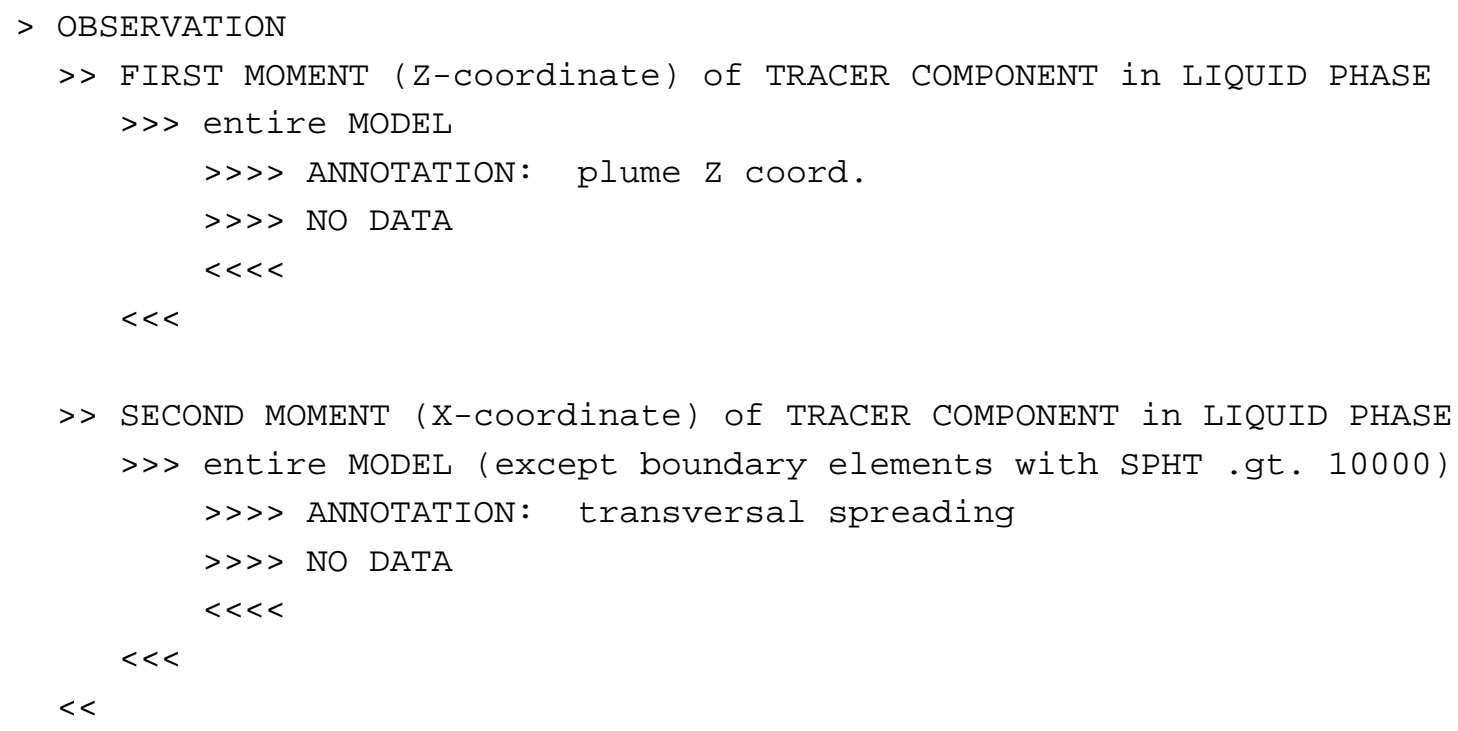

See Also 


\section{Command}

>> MONTE CARLO (SEED: iseed) (GENERATE) (CLASS: nclass)

\section{Parent Command}

>> ERROR

\section{Subcommand}

\section{Description}

This command performs Monte Carlo simulations to determine the uncertainty of model predictions as a result of parameter uncertainty. The general procedure is as follows:

(1) a probability distribution is defined for each uncertain parameter;

(2) parameter values are randomly sampled from their respective distributions;

(3) sampled parameter values are randomly combined to obtain a parameter vector;

(4) a TOUGH2 simulation is performed and the results at the specified observation points are stored;

(5) steps (3) through (4) are repeated;

(6) the ensemble of the modeling results can be statistically analyzed.

The type of distribution can be specified for each parameter in the respective parameter definition block (see command >>> UNIFORM or >>>> NORMAL). The mean is given by the initial guess (see command $>>>>$ GUESS), and the standard deviation by command >>> DEVIATION $(\mathrm{p})$. The generated parameter value is rejected if outside the specified range (see command >>> RANGE).

The number of Monte Carlo simulations, $n_{M C}$, must be provided in block $>>$ CONVERGE using command $>>>$ SIMULATION, i.e., by specifying the maximum number of TOUGH2 simulations. The number of Monte Carlo simulations $n_{M C}$ can be considered sufficient if:

(1) The selected probability density function of the input parameters is reasonably well approximated by the histogram of the randomly generated parameter values. If correlations among the parameters are to be included, use Empirical Orthogonal Functions (command >>> EOF). Keyword GENERATE can be used in combination with various seed numbers (keyword SEED) and values for $n_{M C}$ (command >>> SIMULATION) to generate histograms of the input parameters without actually performing Monte Carlo simulations. Once a satisfactory distribution of the input parameters is achieved, the Monte Carlo simulations can be invoked by simply deleting keyword GENERATE.

(2) The histogram of the model predictions allows for a statistical analysis. That means that a sufficient number of realizations (= simulation results) should fall within each 
interval used to calculate probabilities. For example: The probability that TCE concentrations $c_{T C E}$ fall within the interval $[a, b]$ is approximated by:

$$
\Gamma_{[a, b]}=\operatorname{Pr}\left(a \leq c_{T C E} \leq b\right)=\frac{\text { number of realizations in interval }[a, b]}{\text { total number of Monte Carlo simulations }}=\frac{n_{[a, b]}}{n_{M C}}
$$

Therefore, the minimum number of Monte Carlo simulations, $n_{M C \text {, min }}$, should be large enough so that $\Gamma_{[a, b]}$ remains constant, i.e. independent of $n_{M C}$. This condition is fulfilled for relatively small values of $n_{M C}$ in the case of intervals around the mean, where $n_{[a, b]}$ is usually large due to the high probability density. However, if we are interested in the tail of the distribution, for example to calculate the (low) risk that TCE concentrations exceed a certain standard, then the number of Monte Carlo simulations required is much higher.

(3) The minimum number of Monte Carlo simulations must be increased if the number of uncertain parameter increases because more parameter combinations are possible.

(4) From experience, the number of Monte Carlo simulations can be as low as 50 and as high as 2000 or greater.

Histograms of the input parameters and output variables as printed to the iTOUGH2 output file. By default, the interval between the smallest and highest value is subdivided into $\sqrt{n_{M C}}$ classes. The number of classes can be changed using keyword CLASS.

The standard plot file contains all output sets from $n_{M C}$ simulations, where each set contains the model result as a function of time. A second plot file is generated with "_ $m c$ " in the file name. In this second plot file, each curve represents the ensemble of the model output at one point in time. Denoting the number of output sets, for which prediction uncertainty is to be studied, by $n_{\text {set }}$, and the number of times by $n_{\text {times }}$, then the standard plot contains $n_{\text {set }} \cdot n_{M C}$ curves, each curve consisting of $n_{\text {times }}$ points. The second plotfile contains $n_{\text {set }} \cdot n_{\text {times }}$ curves, each consisting of $n_{M C}$ points. 


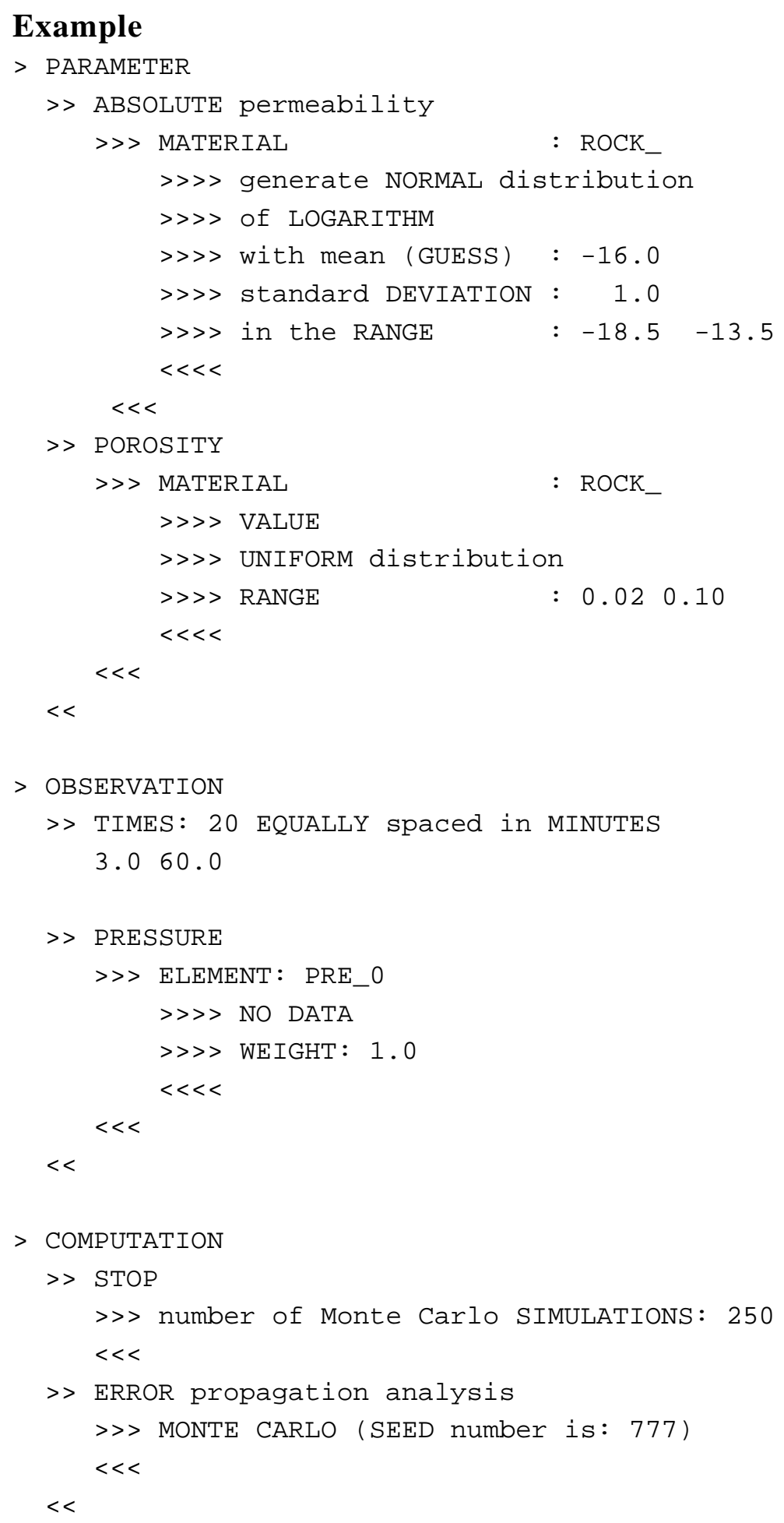

\section{See Also}

>> EMPIRICAL ORTHOGONAL FUNCTIONS, $>>>$ FOSM, $>>>$ SIMULATION, $>>>$ NORMAL, $>>>>$ RANGE, $>>>>$ UNIFORM 


\section{Command}

>> NEW OUTPUT

\section{Parent Command}

>> OUTPUT

\section{Subcommand}

\section{Description}

This command creates a new TOUGH2 output file with unique file name for each TOUGH2 run. By default, the TOUGH2 output file is overwritten each time a new simulation starts.

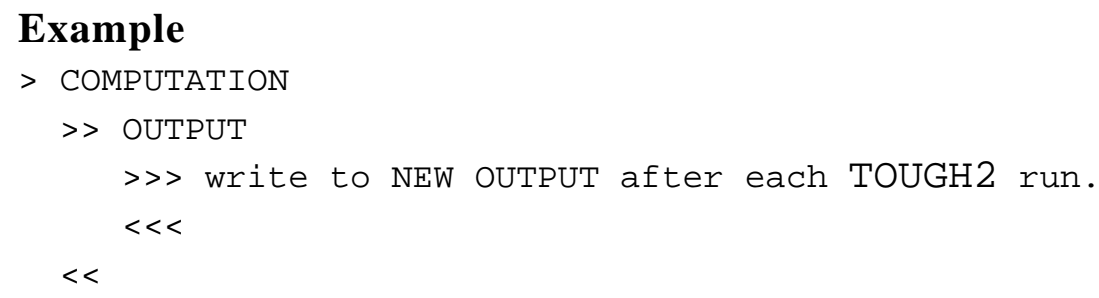

See Also 


\section{Command}

$>>$ NONE

\section{Parent Command}

all second-level commands in block > PARAMETER or > OBSERVATION addressing parameters or observations that refer to the entire model domain or a non-specific item.

\section{Subcommand}

all fourth-level commands in block > PARAMETER or > OBSERVATION, respectively.

\section{Description}

(synonym for command >> MODEL)

\section{Example}

(see command >> MODEL)

\section{See Also}

$>>$ MODEL 
Command

>>> NORMAL

Parent Command

all third-level commands in block > PARAMETER

Subcommand

Description

(synonym for command $>>>$ GAUSS)

Example

(see command $>>>>$ GAUSS)

See Also

>>> GAUSS 


\section{Command}

>> OBJECTIVE

\section{Parent Command}

>> OUTPUT

\section{Subcommand}

\section{Description}

This command prints the value of the objective function after each TOUGH 2 simulation. By default, the value of the objective function is printed to the output file only after completion of an iTOUGH2 iteration.

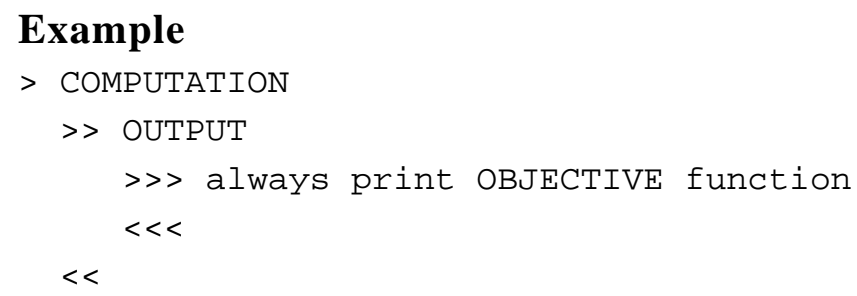

See Also

>> JACOBIAN 


\section{Command}

>> OBJECTIVE: ninvall (ninvali...) (UNSORTED)

\section{Parent Command}

$>$ OPTION

Subcommand

Description

(synonym for command >>> GRID SEARCH)

Example

(see command $>>$ SRID SEARCH)

See Also

>> GRID SEARCH 


\title{
Command
}

$>$ OBSERVATION

\section{Parent Command}

\author{
Subcommands \\ $>$ CONCENTRATION \\ $>>$ CONTENT \\ $>>$ COVARIANCE \\ $>>$ CUMULATIVE \\ $>>$ DRAWDOWN \\ $>>$ ENTHALPY \\ $>>$ FLOW \\ $>>$ GENERATION \\ $>$ LIST \\ $>>$ MASS FRACTION \\ $>$ MOMENT \\ $>>$ PRESSURE \\ $>>$ PRODUCTION \\ $>>$ RESTART TIME \\ $>$ SECONDARY \\ $>>$ SATURATION \\ $>>$ TEMPERATURE \\ $>$ TIME (O) \\ $>>$ TOTAL MASS \\ $>>$ USER (O) \\ $>$ VOLUME
}

\section{Description}

This is the first-level command for specifying the observations available for use in parameter estimation. It can also be used to specify the points in space and time at which output is requested for sensitivity analysis, uncertainty propagation analysis, or plotting. The observations are a subset of all TOUGH2 output variables. Only those variables should be specified for which data are available. Besides the observations listed above, the user can specify additional data types by means of command >> USER.

The second-level command >> TIME is used to select calibration points in time. The observation type is specified by the second-level command, the points in space are identified by the third-level command, and further specifications as well as the data themselves are given through fourth-level commands. The generic structure of a observation block is as follows: 


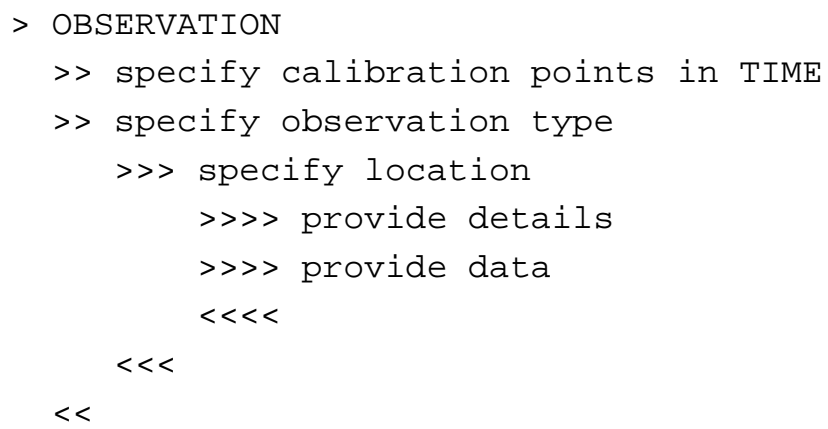

\section{Example}

(see examples on second command level)

\section{See Also}




\section{Command}

>> OPTION

Parent Command

$>$ COMPUTATION

\section{Subcommand}

$>>>$ ANDREWS

$>>$ ANNEAL

$>>$ CAUCHY

$>>>$ DIRECT

$\gg>$ FORWARD

$>>>$ GAUSS-NEWTON

$>>>$ GRID SEARCH

$>>$ L1-ESTIMATOR

$>>>$ LEAST-SQUARE

$>>>$ LEVENBERG-MARQUARDT

$>>$ LIST

$>>>$ OBJECTIVE

$>>>$ QUADRATIC-LINEAR

$>>$ SELECT

$>>$ SENSITIVITY

$>>$ SIMPLEX

$>>$ STEADY-STATE

\section{Description}

This is the parent command of a number of subcommands for selecting iTOUGH2 program options. By default, iTOUGH2 performs automatic model calibration using the weighted least-squares objective function and the Levenberg-Marquardt minimization algorithm.

\section{Example}

(see examples on third command level)

See Also 


\section{Command}

>> OUTPUT

\section{Parent Command}

$>$ COMPUTATION

\section{Subcommand}

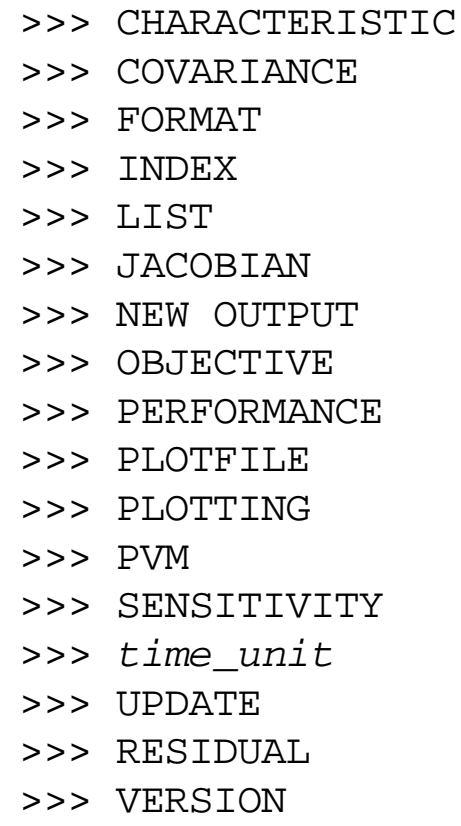

\section{Description}

This command specifies the format and amount of printout generated. The iTOUGH2 output file contains the results usually needed for subsequent interpretation. Additional information can be requested which may be useful for debugging.

\section{Example}

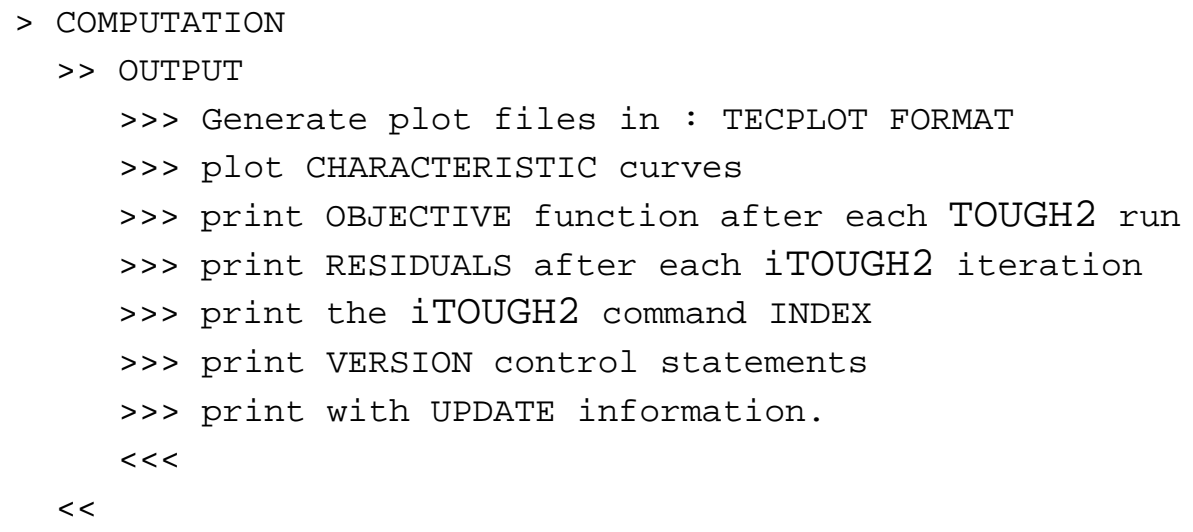

See Also

- 


\section{Command}

>> PARALLEL PLATE

\section{Parent Command}

$>$ PARAMETER

\section{Subcommand}

$>>$ MATERIAL

\section{Description}

This command selects as a parameter the aperture of a parallel plate fracture model. The single fracture must be modeled as a 1D- or 2D model of thickness 1 meter, i.e., aperture must be identical to porosity. Furthermore, the capillary pressure model must contain a parameter $\mathrm{CP}(2, \mathrm{NMAT})$ which represents the gas entry pressure (e.g., Brooks-Corey $(\mathrm{ICP}=10)$, van Genuchten $(\mathrm{ICP}=11)$ ).

The parallel plate model relates porosity $\varphi$, permeability $k\left[\mathrm{~m}^{2}\right]$, and gas entry pressure $p_{e}$ $[\mathrm{Pa}]$ to aperture $a[\mathrm{~m}]$ as follows:

$$
\begin{gathered}
\varphi=a \\
k=\frac{f \cdot a^{3}}{12} \\
p_{e}=\frac{2 \sigma \cos (\phi)}{a}=\frac{0.14366}{a}
\end{gathered}
$$

Here, $f=1$ is fracture frequency $\left[\mathrm{m}^{-1}\right], \sigma=0.07183[\mathrm{~Pa} \cdot \mathrm{m}]$ is the surface tension of water, and $\phi$ is the contact angle which is assumed to be zero. Using this option overwrites the porosity, absolute permeability and parameter $\mathrm{CP}(2, \mathrm{NMAT})$ given in the TOUGH2 input file.

Note that the parallel plate model used here is inconsistent. Using a relative permeability and capillary pressure function presumes some surface roughness or aperture distribution within the fracture plane.

\section{Example}

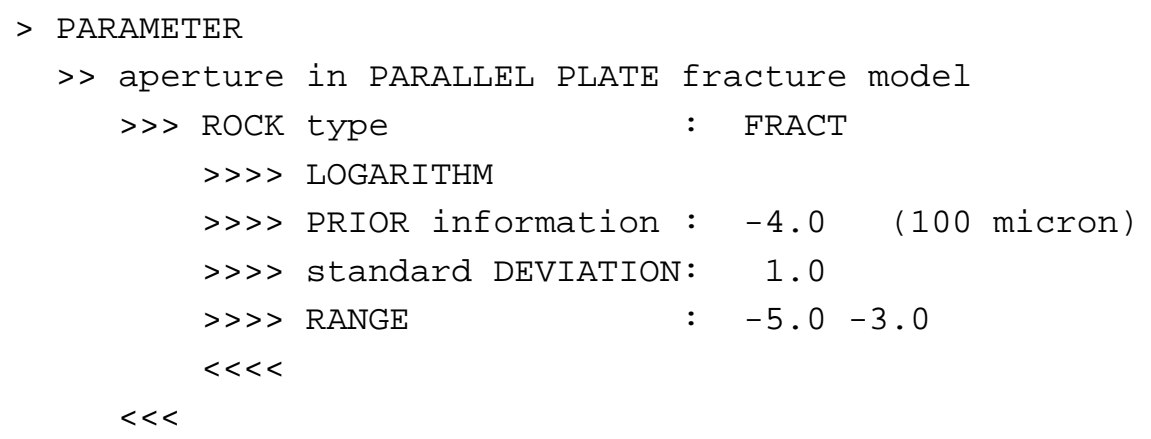

See Also 


\section{Command}

$>$ PARAMETER

\section{Parent Command}

-

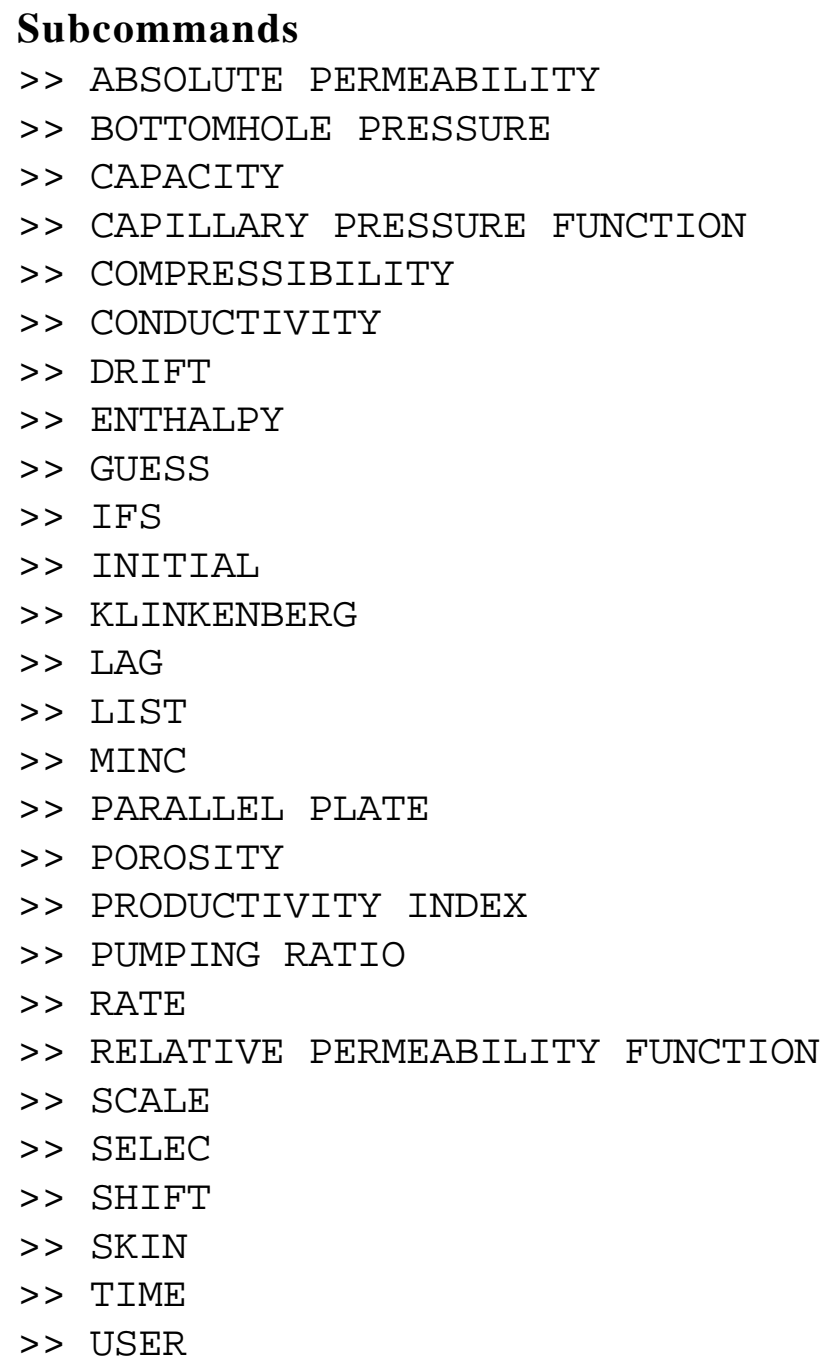

\section{Description}

This is the first-level command to specify the parameters to be estimated. The parameters to be estimated are a subset of all TOUGH 2 input parameters defined in the TOUGH 2 input file. Only those parameters should be specified that are unknown or uncertain; they will be subjected to the estimation process or uncertainty propagation analysis. Select only those parameters that are sensitive enough to influence the state variables for which observations are available (see also $>>>$ SELECT). Besides the parameters listed above, the user can specify additional parameters by means of command $>>$ USER. 
The parameter type is specified by the second-level command, the domain it refers to is identified by the third-level command, and further specifications must be provided through a number of fourth-level commands. The generic structure of a parameter block is as follows:

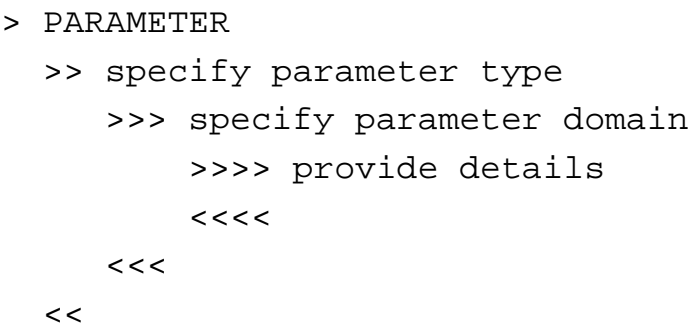

\section{Example}

(see examples for second-level commands)

\section{See Also}

$>$ OBSERVATION, > COMPUTATION 


\section{Command}

>>> PARAMETER: index (index_i...)

\section{Parent Command}

all third-level commands in block > PARAMETER and > OBSERVATION

\section{Subcommand}

$-$

\section{Description}

(synonym for command $>>>>$ INDEX)

\section{Example}

(see command $>>>>$ INDEX)

See Also

$>>>$ INDEX 


\section{Command}

>> PERFORMANCE

\section{Parent Command}

>> OUTPUT

\section{Subcommand}

$-$

\section{Description}

This command performs a very rough benchmark analysis of computer performance and prints the relative CPU time requirement as compared to a reference workstation.

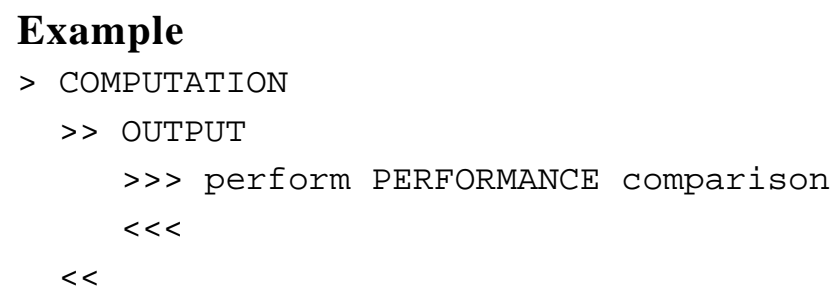

See Also 


\section{Command}

>>> PERTURB: (-) alpha $(\%)$

Parent Command

$>>$ JACOBIAN

\section{Subcommand}

$-$

\section{Description}

This command specifies the perturbation factor $\alpha$ for numerical computation of the Jacobian matrix.

The columns of the Jacobian matrix are calculated by perturbing the corresponding parameter $p_{i}$ by a small amount $\delta p_{i}$, and taking either a forward or centered finite difference quotient. The perturbation is usually a fraction of the parameter value itself:

$$
\delta p_{i}=\alpha \cdot p_{i}
$$

The default value for $\alpha$ is $1 \%$, which can be changed globally for all parameters using the third-level command $>>>$ PERTURB, or individually for each parameter using the fourthlevel command $>>>>$ PERTURB. A negative value can be provided to specify a constant perturbation, independent of parameter value:

$$
\delta p_{i}=|-\alpha|
$$

This option is sometimes required for parameters having either very small or very large values (such as initial pressure, generation times, or residual gas saturation).

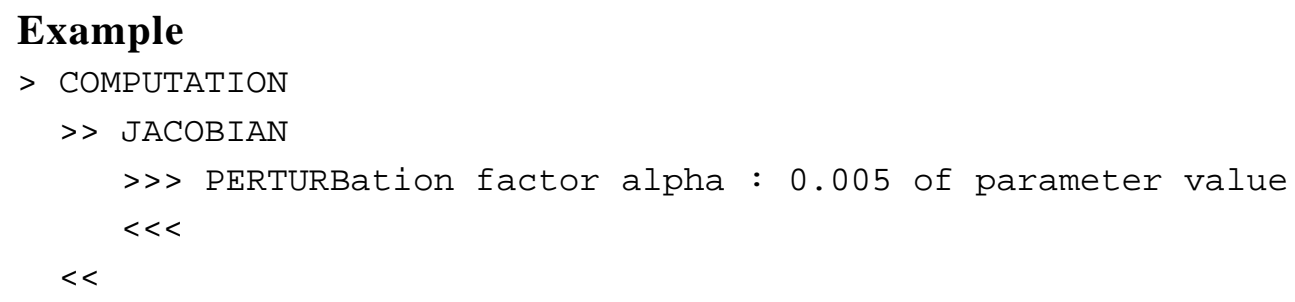

See Also

>>> FORWARD, >>> CENTERED, >>>> PERTURB 


\section{Command}

>>> PERTURB: (-) alpha (\%)

Parent Command

all third-level commands in block > PARAMETER

\section{Subcommand}

\section{Description}

This command specifies the perturbation factor $\alpha$ for numerical computation of the Jacobian matrix. The columns of the Jacobian matrix are calculated by perturbing the corresponding parameter $p_{i}$ by a small amount $\delta p_{i}$, and taking either a forward or centered finite difference quotient. The perturbation is usually a fraction of the parameter value itself:

$$
\delta p_{i}=\alpha \cdot p_{i}
$$

The default value for $\alpha$ is $1 \%$, which can be changed globally for all parameters using the third-level command >>> PERTURB, or individually for each parameter using the fourthlevel command >>>> PERTURB. A negative value can be provided to specify a constant perturbation, independent of parameter value:

$$
\delta p_{i}=|-\alpha|
$$

This option is sometimes required for parameters having either very small or very large values (such as initial pressure, generation times, or residual gas saturation).

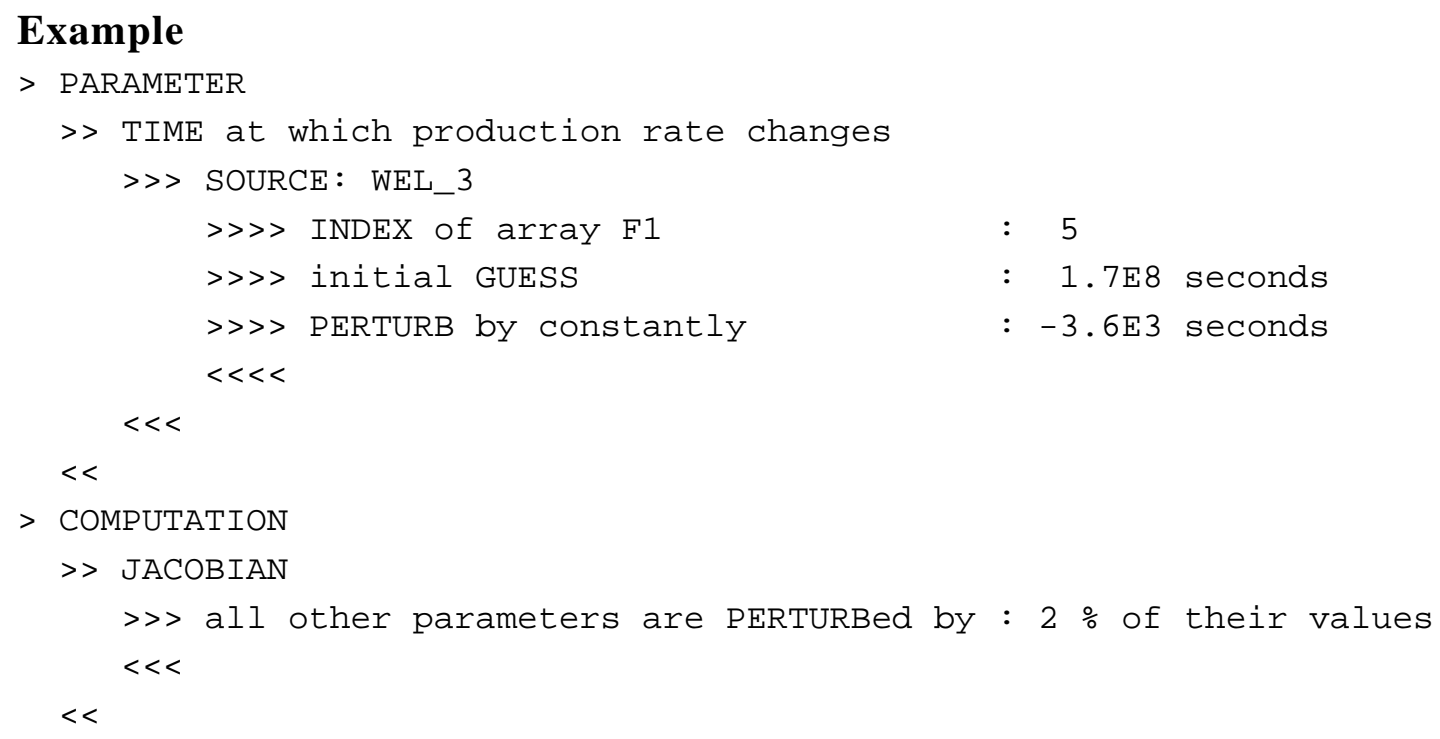

See Also

$>>>$ PERTURB 


\section{Command}

>>> PHASE phase_name/: iphase

\section{Parent Command}

$>>$ ELEMENT

$>>$ SOURCE (O)

\section{Subcommand}

$-$

\section{Description}

This command identifies a phase either by its name (phase_name) or the phase number (iphase). A list of allowable phase names for the given EOS module can be obtained from the header of the iTOUGH2 output file.

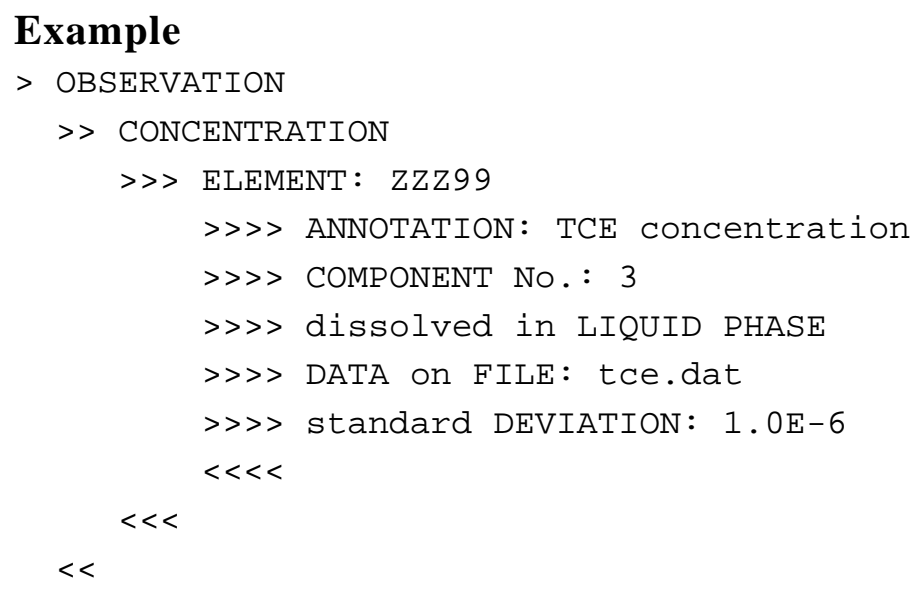

See Also

$>>>$ COMPONENT 


\section{Command}

>>>> PICK: npick

\section{Parent Command}

all third-level commands in block > OBSERVATION

\section{Subcommand}

$-$

\section{Description}

This command identifies the number of data points being skipped when reading from a long data file. Only every npick data point is read. The default is $n p i c k=1$, i.e., every data point is accepted.

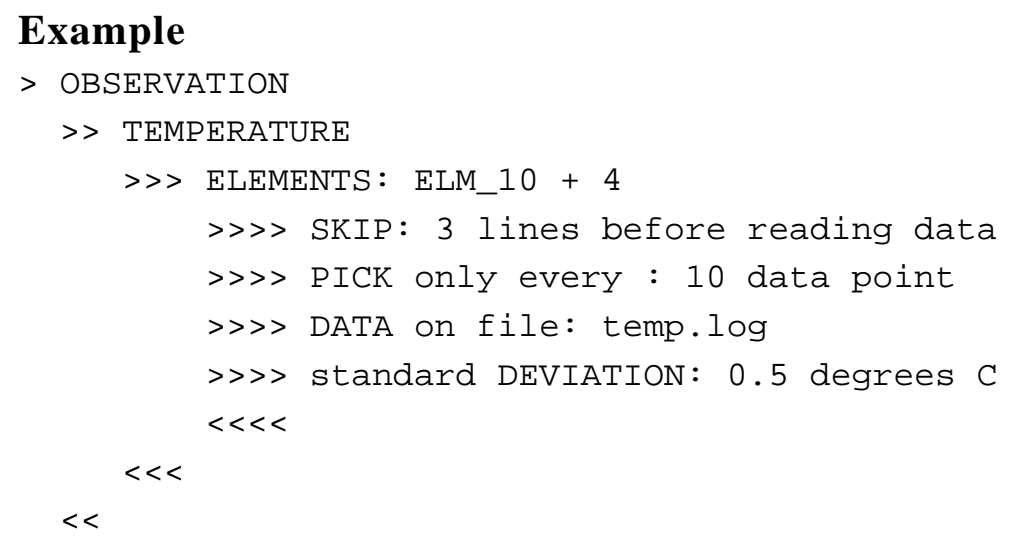

See Also

>>> COLUMN, >>> DATA, >>> FORMAT, >>> SET (0), >>> SKIP 


\section{Command}

>> PLOTFILE: format (LIST)

\section{Parent Command}

>> OUTPUT

Subcommand

Description

(synonym for command >> FORMAT (o))

Example

(see command $>>$ FORMAT (o))

See Also

$>>$ FORMAT (O) 


\section{Command}

>> PLOTTING: niter

\section{Parent Command}

$>$ OUTPUT

\section{Subcommand}

$-$

\section{Description}

By default, the plot file contains the observed data (interpolated at the calibration points), as well as the system response calculated with the initial and final parameter set. Additional intermediate curves can be requested for visualizing the optimization process. Curves are generated for every multiple of niter iTOUGH2 iterations.

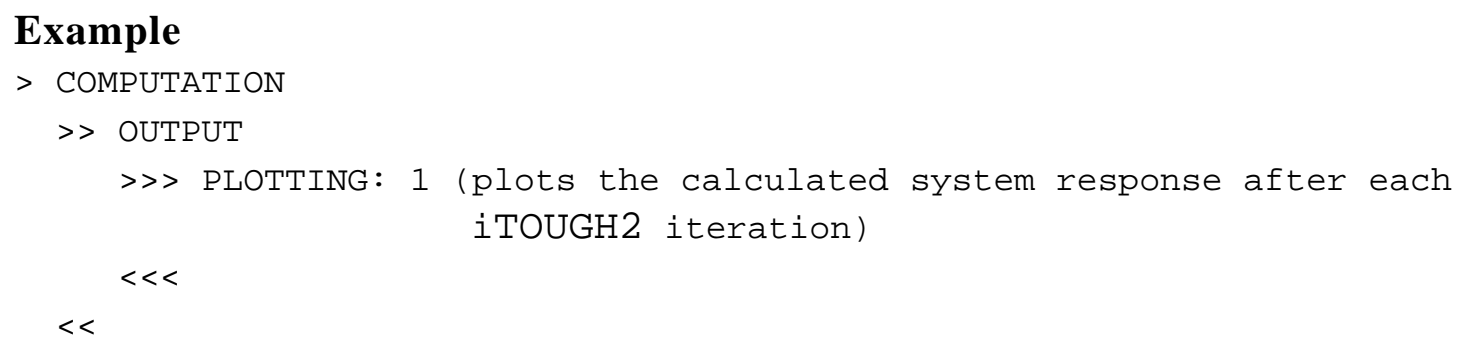

See Also 


\section{Command}

>>> POLYNOM: idegree (time_unit)

\section{Parent Command}

all third-level commands in block > OBSERVATION

\section{Subcommand}

$-$

\section{Description}

Represents observed data by a polynomial of degree idegree:

$$
z^{*}(t)=\sum_{i=0}^{\text {idegree }} A_{i} \cdot t^{i}
$$

where $t$ denotes time in time_units (default is seconds), and $A_{i}$ are the idegree +1 coefficients which are read in free format on the lines following the command line.

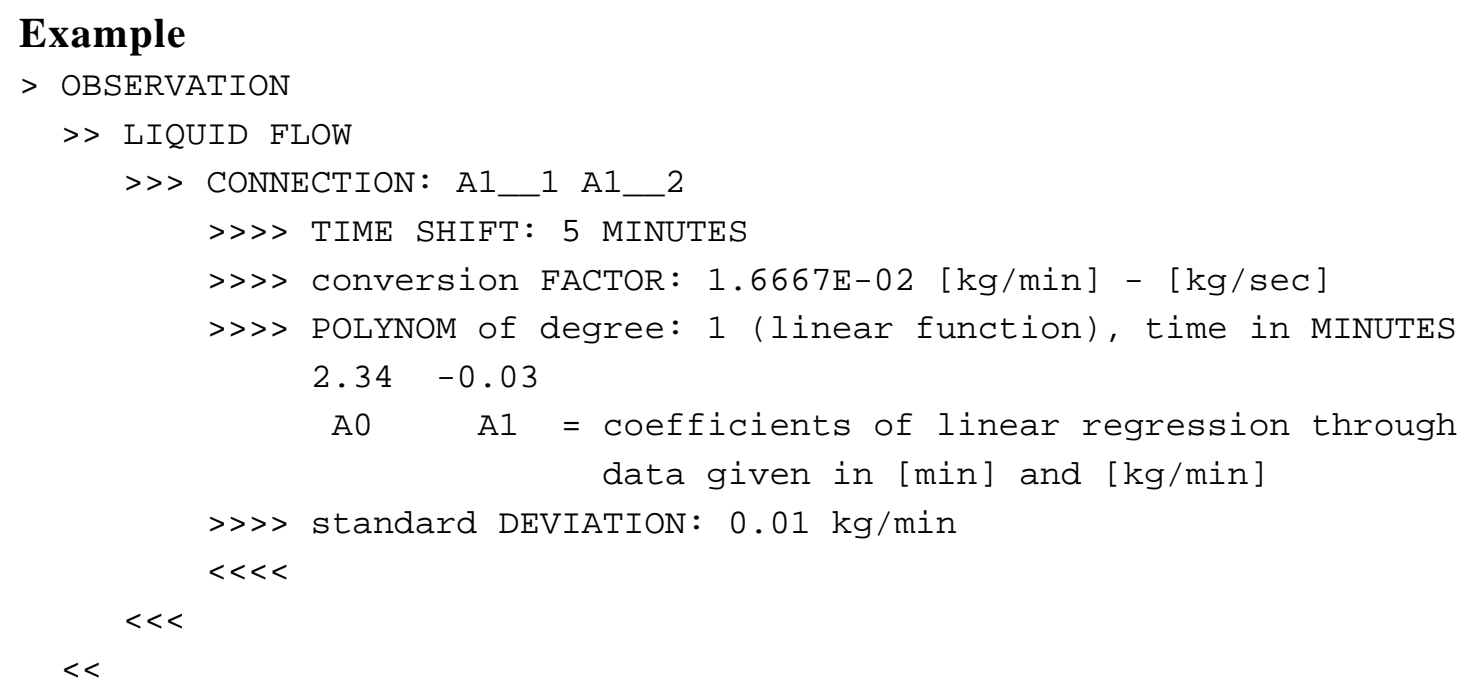

See Also

>>>> DATA, >>>> USER 


\section{Command}

>> POROSITY

\section{Parent Command}

$>$ PARAMETER

\section{Subcommand}

>> MATERIAL

\section{Description}

This command selects as a parameter porosity (TOUGH2 variable POR (NMAT)). This parameter refers to a rock type.

Note that the porosity specified in TOUGH2 block ROCKS (variable POR (NMAT)) may be overwritten by non-zero values given in block INCON (variable PORX). However, porosity values are not overwritten if the element belongs to a rock type for which porosity is a parameter to be estimated, unless a negative value for $P O R X$ is provided.

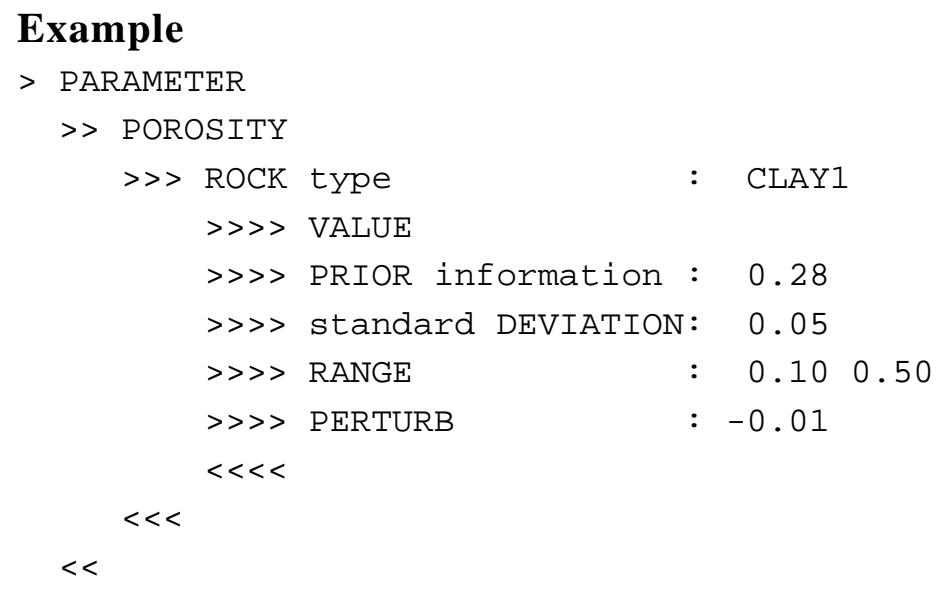

See Also

- 


\section{Command}

>> POSTERIORI

\section{Parent Command}

$>$ ERROR

\section{Subcommand}

$-$

\section{Description}

The estimated error variance $s_{0}^{2}$ represents the variance of the mean weighted residual and is thus a measure of goodness-of-fit:

$$
s_{0}^{2}=\frac{\mathbf{r}^{T} \mathbf{C}_{z z}^{-1} \mathbf{r}}{m-n}
$$

The a posteriori error variance $s_{0}^{2}$ or a priori error variance $\sigma_{0}^{2}$ is used in the subsequent error analysis. For example, the covariance matrix of the estimated parameters, $\mathbf{C}_{p p}$, is directly proportional to the scalar $s_{0}^{2}$ or $\sigma_{0}^{2}$, respectively. Note that if the residuals are consistent with the distributional assumption about the measurement errors (i.e., matrix $\mathbf{C}_{z z}$ ), then the estimated error variance assumes a value close to one.

The user must decide whether the error analysis should be based on the a posteriori or $a$ priori error variance. The decision can also be delegated to the Fisher Model Test (see command $>>>$ FISHER). iTOUGH2 uses the a posteriori error variance $s_{0}^{2}$ by default.

\section{Example}

$>$ COMPUTATION

$>>$ ERROR analysis

$>>$ based on A POSTERIORI error variance

$<<$

See Also

$>>$ FISHER, $>>>$ PRIORI 


\section{Command}

>> PRESSURE (CAPILLARY) (phase_name/PHASE: iphase)

\section{Parent Command \\ $>$ OBSERVATION}

\section{Subcommand}

$>>$ ELEMENT

\section{Description}

This command selects as an observation type the phase pressure of capillary pressure. This observation type refers to one or more elements. The phase name phase_name or phase number iphase, which depend on the EOS module being used, are listed in the header of the iTOUGH2 output file. They can be specified either on the command line or using subcommand $>>>>$ PHASE. If no phase is specified, iTOUGH2 takes the pressure of the first phase which is usually the reference pressure. The pressure of a given phase is calculated as the sum of the reference pressure and the corresponding capillary pressure.

In a two-phase system, there is only one capillary pressure. The capillary pressure can be selected using keyword CAP ILLARY. In a three-phase system (e.g., gas, NAPL, aqueous phase), there are two capillary pressures, i.e., the capillary pressure between the NAPL and the gas phase, and between the aqueous and the gas phase, which is the reference phase. The name of the wetting phase must be provided to identify the particular capillary pressure in the three-phase system.

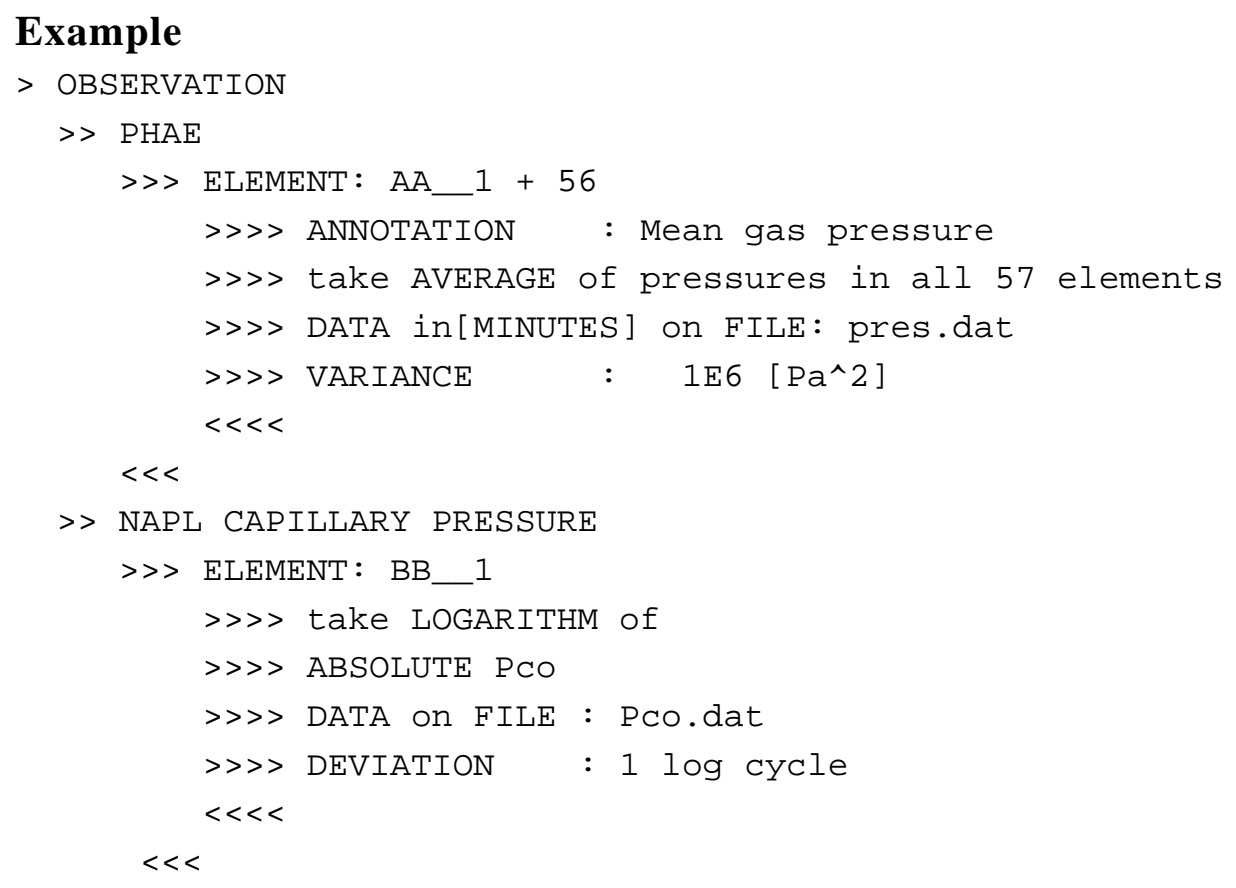

See Also

>> DRAWDOWN 


\section{Command}

>>> PRIOR: prior_info

Parent Command

all third-level commands in block > PARAMETER

\section{Subcommand}

\section{Description}

This command provides the prior information of the parameter to be estimated. If command $>>>>$ PRIOR is omitted, the value from the TOUGH2 input file is taken. Prior information is only effective if a standard deviation is provided (see command $>>>>$ DEVIATION (p) ), in which case the difference between the parameter estimate and its prior value is penalized in the objective function. The starting point for the optimization is given through commands $>>$ GUESS and $>>>>$ GUESS.

If command $>>>>$ LOGARITHM is present, the prior information is the logarithm of the parameter. Similarly, if command $>>>>$ FACTOR is present, the prior information should be a multiplication factor (default is 1.0 ).

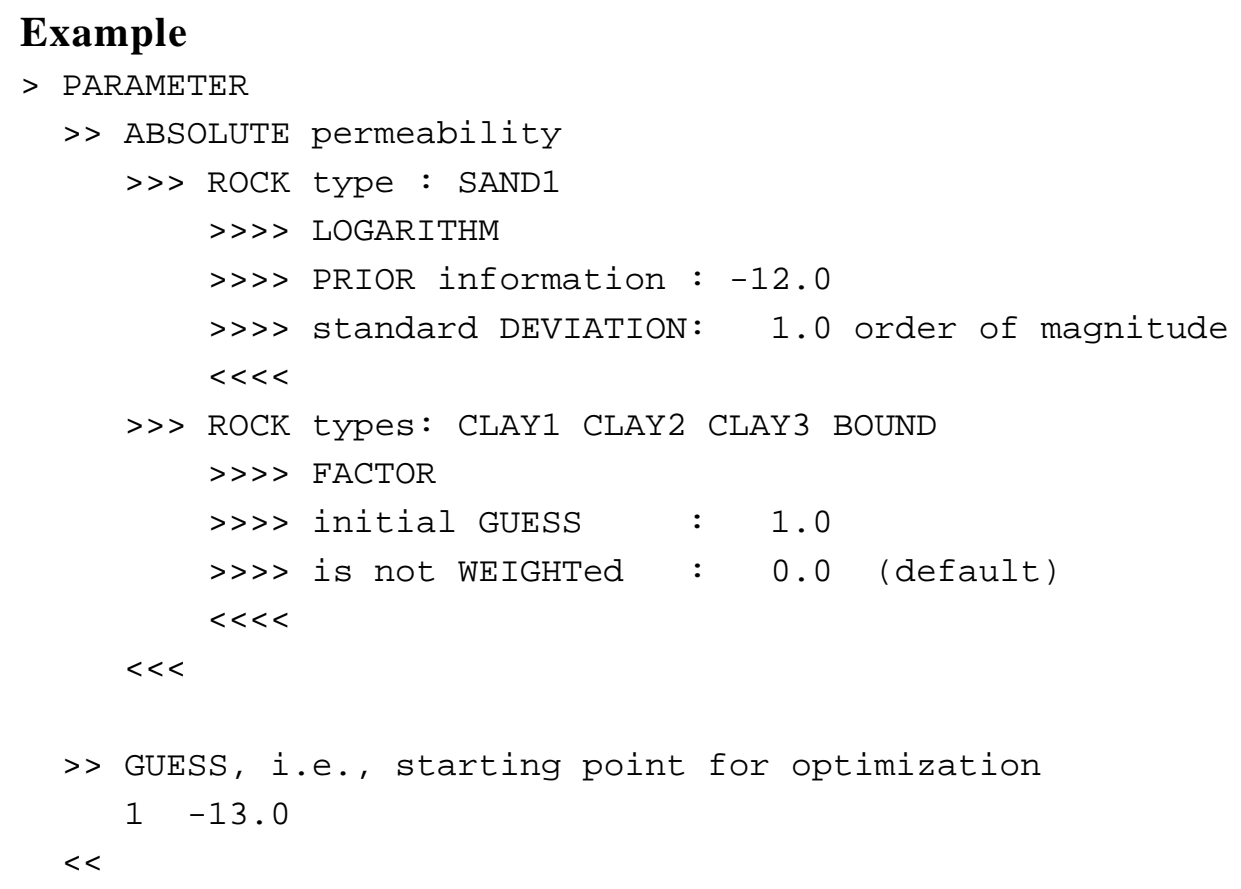

See Also

>> GUESS, >>> GUESS, >>>> DEVIATION, >>> VARIATION 


\section{Command}

$>>$ PRIORI

\section{Parent Command}

$>$ ERROR

\section{Subcommand}

$-$

\section{Description}

The estimated error variance $s_{0}^{2}$ represents the variance of the mean weighted residual and is thus a measure of goodness-of-fit:

$$
s_{0}^{2}=\frac{\mathbf{r}^{T} \mathbf{C}_{z z}^{-1} \mathbf{r}}{m-n}
$$

The a posteriori error variance $s_{0}^{2}$ or a priori error variance $\sigma_{0}^{2}$ is used in the subsequent error analysis. For example, the covariance matrix of the estimated parameters, $\mathbf{C}_{p p}$, is directly proportional to the scalar $s_{0}^{2}$ or $\sigma_{0}^{2}$, respectively. Note that if the residuals are consistent with the distributional assumption about the measurement errors (i.e., matrix $\mathbf{C}_{z z}$ ), then the estimated error variance $s_{0}$ assumes a value close to one.

The user must decide whether the error analysis should be based on the a posteriori or $a$ priori error variance. The decision can also be delegated to the Fisher Model Test (see command $>>>$ FISHER). iTOUGH2 uses the a posteriori error variance $s_{0}^{2}$ by default. However, for design calculations or synthetic inversions, the error analysis should be based on the a priori variance $\sigma_{0}^{2}$ which is 1 by definition.

\section{Example}

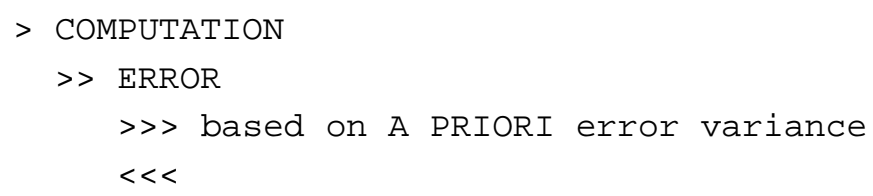

See Also

>> FISHER, >> POSTERIORI 
Command

>> PRODUCTION (phase_name/PHASE: iphase)

Parent Command

> OBSERVATION

Subcommand

>> SINK

Description

(synonym for command >> GENERATION)

Example

(see command >> GENERATION)

See Also

>> GENERATION 


\section{Command}

>> PRODUCTIVITY INDEX

\section{Parent Command}

> PARAMETER

\section{Subcommand}

>> SOURCE

\section{Description}

This command selects the productivity index for wells on deliverability (TOUGH2 variable $G X)$ as a parameter to be estimated. This parameter refers to a sink/source code name. The generation type must be DELV.

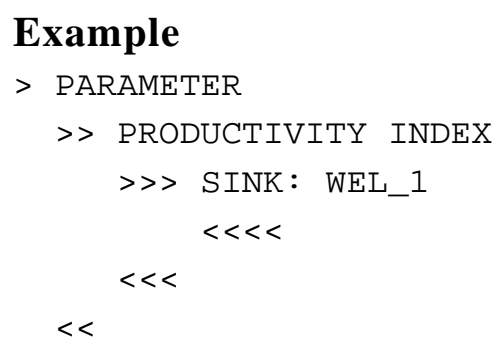

See Also

$-$ 


\section{Command}

>> PUMPING RATIO

\section{Parent Command}

$>$ PARAMETER

\section{Subcommand}

>> SOURCE

\section{Description}

This command selects as a parameter the pumping ratios of all wells belonging to the same well group. This option can be used to determine the distribution of generation rates among a group of wells, e.g., to determine the optimum pumping strategy for a cleanup operation. For example, the total extraction rate from a system of wells is often limited by the treatment capacity. However, extraction rates of individual wells can be adjusted, optimizing the efficiency of the cleanup operation. A well group consists of all sinks/sources with the same code name (TOUGH2 variable $S L$ ). The sum of the constant generation rates (TOUGH2 variable $G X$ ) of all wells within the same well group remains constant, but individual rates are adjusted. It is imperative to estimate as many pumping ratios as the number $n$ of wells within the well group of interest. After optimization, the actual generation rate for well $i$ is calculated from the prescribed total pumping rate $q_{t o t}$ and the estimated pumping ratio $a_{i}$

$$
q_{i}=q_{\text {tot }} \frac{a_{i}}{\sum_{j=1}^{n} a_{j}}
$$

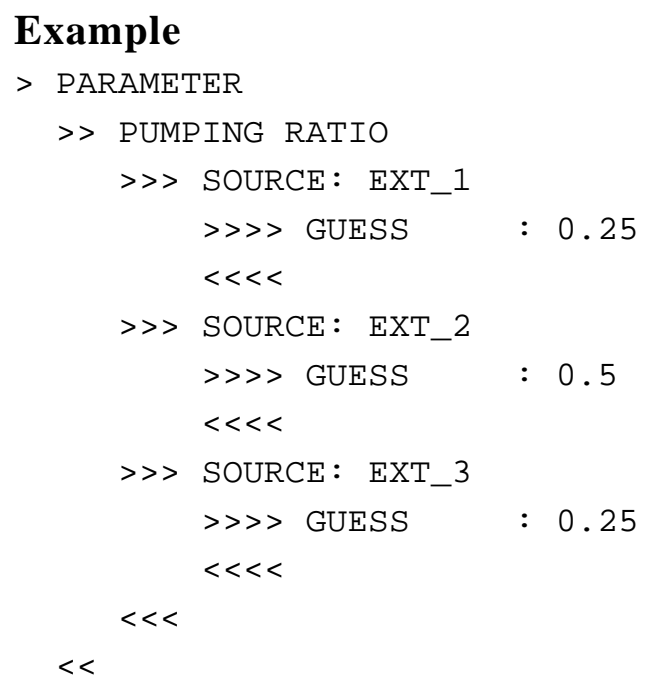

See Also 


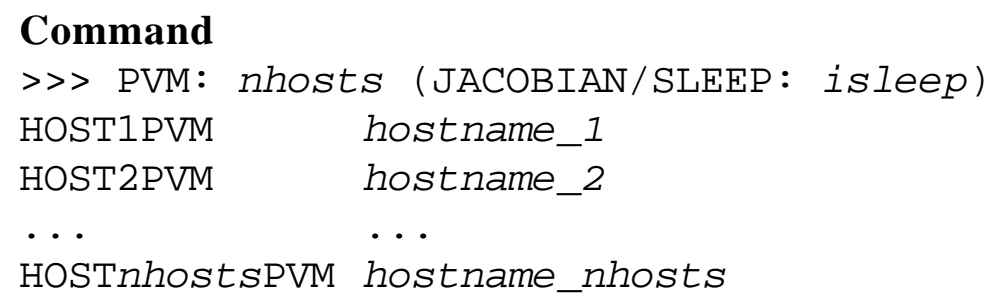

\section{Subcommand}

\section{Description}

Certain TOUGH2 forward calculations are independent from each other and can thus be processed in parallel on a heterogeneous network of UNIX workstations using PVM (Parallel Virtual Machine, Geist et al. [1994]). Consult Finsterle [1998] for details about installation and execution of iTOUGH2-PVM applications.

The number of hosts nhosts is provided on the command line, followed by nhosts lines containing the keyword ${ }^{*}$ HOSTi $\quad(i=1, \ldots$, nhosts $)$ and the name of the host. The wild card * must be a unique identifier if more than one iTOUGH2-PVM application are run simultaneously. The master process must not be included in the list. However, a child process may be spawned on the parent host. The host must be registered in file .rhosts. The name of the host must be identical to that appended to the file name of the iTOUGH2 executable on that specific machine. A host (especially the multiprocessor machines) may be named several times in the list of hosts.. Notice that the PVM block in the iTOUGH2 input file is also parsed by the $i$ TOUGH2 script [Finsterle, 1998]. If the Levenberg-Marquardt algorithm is used, parallelization can be restricted to the evaluation of the Jacobian matrix by using keyword JACOBIAN on the command line. The parent process can be suspended for is leep seconds (default: is leep=1) before it checks for incoming data.

\section{Example}

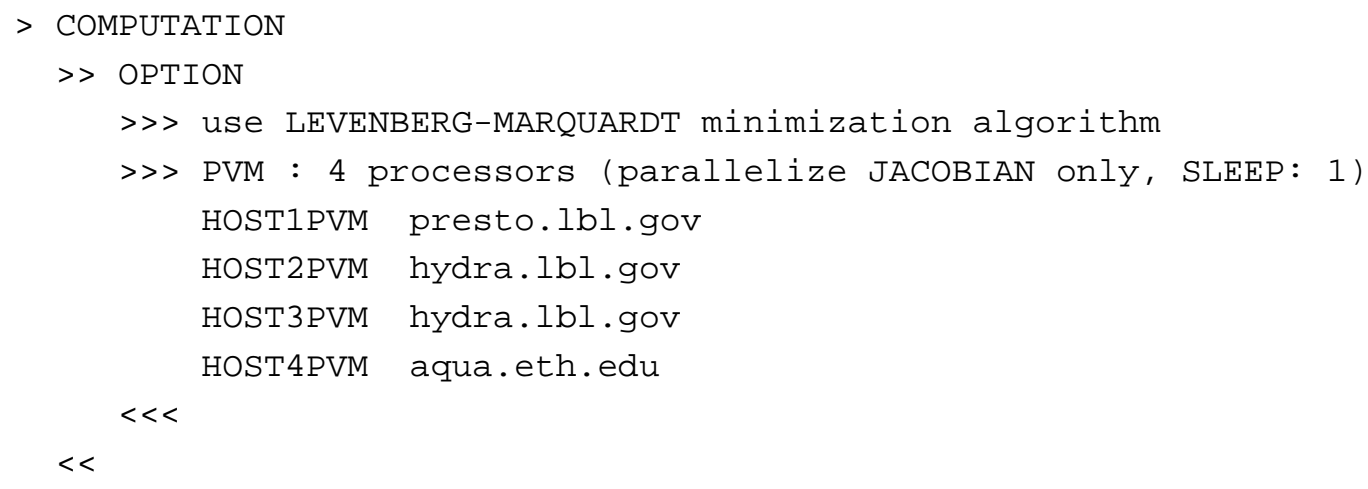

See Also 


\section{Command}

>> QUADRATIC-LINEAR: C

\section{Parent Command}

>> OPTION

\section{Subcommand}

$-$

\section{Description}

This command selects a quadratic-linear robust estimator. Given this estimator, the objective function to be minimized is a combination of least-squares for small residuals and the first norm for residuals larger than $c$-times the prior standard deviation:

$$
S=\sum_{i=1}^{m} \gamma\left(y_{i}\right)
$$

where

with

$$
\gamma\left(y_{i}\right)=\left\{\begin{array}{cl}
y_{i}^{2} & \left|y_{i}\right| \leq c \\
c\left(2\left|y_{i}\right|-c\right) & \left|y_{i}\right|>c
\end{array}\right.
$$

$$
y_{i}=\frac{r_{i}}{\sigma_{i}}
$$

This objective function does not correspond to a standard probability density function. It has the general characteristic that the weight given individual residuals first increases quadratically with deviation, then only linearly to reduce the impact of outliers.

For $c \rightarrow \infty$, the estimator is identical to least-squares; for $c \rightarrow 0$, it approaches the $\mathrm{L}_{1^{-}}$ estimator.

Note that this objective function is minimized using the standard Levenberg-Marquardt algorithm which is designed for a quadratic objective function. Since the function is quadratic for $y_{i} \leq c$, the Levenberg-Marquardt algorithm is usually quite efficient.

\section{Example}

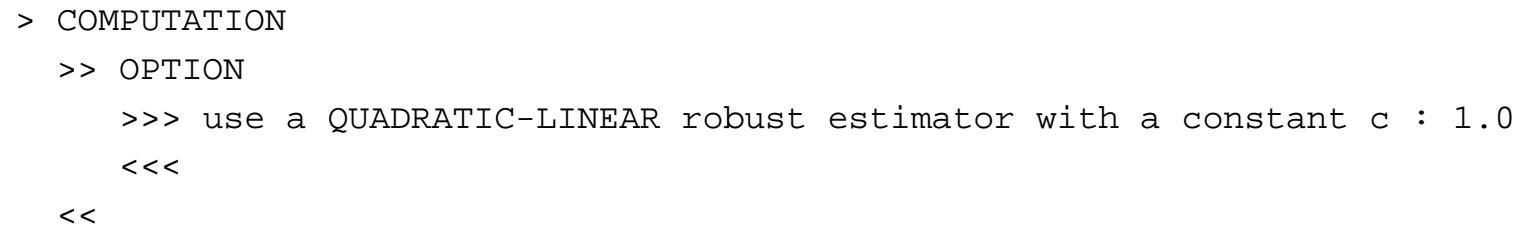

See Also

>> ANDREWS, >> CAUCHY, >>> L1-ESTIMATOR, >> LEAST-SQUARES 


\section{Command}

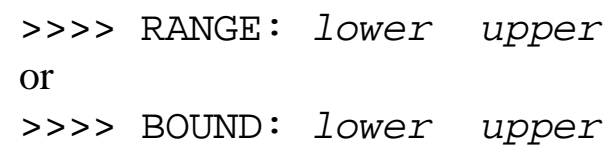

\section{Parent Command}

all third-level commands in block > PARAMETER

\section{Subcommand}

\section{Description}

This command sets the admissible parameter range. It provides lower and upper bounds of the parameter. During the optimization process, iTOUGH2 may suggest parameter values that are physically not valid (e.g., negative porosity) or not reasonable (e.g., very high permeability). Limiting the admissible range for the values a parameter can assume prevents the simulation from stopping due to an unphysical or unreasonable parameter value. However, it is strongly suggested not to specify a narrow parameter range about the initial guess. The range should reflect physical bounds, and the expected variation of the parameter. If prior knowledge suggests that a certain parameter varies only slightly about the initial guess, this information should enter the inversion as a standard deviation associated with the initial guess, i.e. prior information (see command $>>>>$ DEVIATION $(p)$ ). A parameter tends to greatly vary, potentially hitting its lower or upper bound, if (i) a systematic error is present, (ii) the initial guess is far away from the best estimate, (iii) the parameter is not sensitive, or (iv) the parameter is highly correlated to more sensitive parameters. The final parameter set should not contain parameters at their lower or upper bounds. If some of the estimated parameters are at the bounds, it is suggested to carefully examine the four potential reasons mentioned above. A new inversion should be performed after corrective actions have been taken.

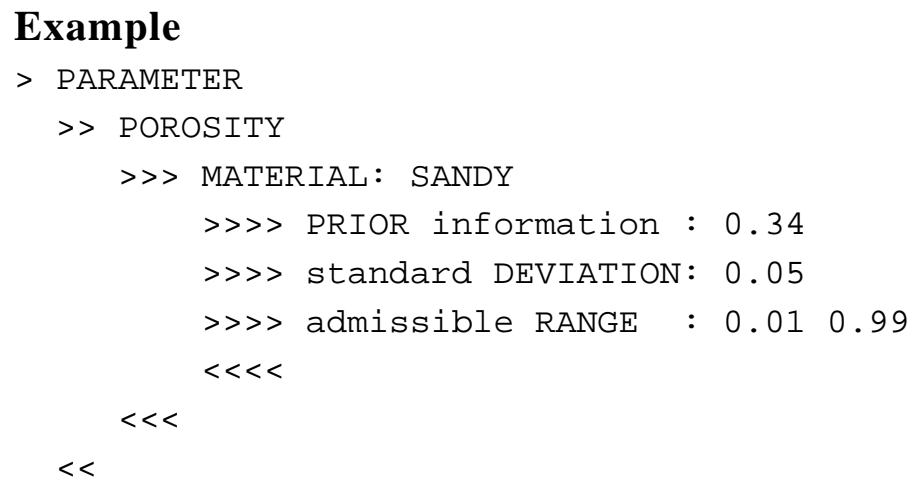

See Also

>>> DEVIATION (p), >>> STEP 


\section{Command}

$>$ RATE

\section{Parent Command}

$>$ PARAMETER

\section{Subcommand}

$>>$ SOURCE

\section{Description}

This command selects as a parameter the constant generation rate (TOUGH2 variable GX) or time-dependent generation rate (TOUGH2 variable $F 2(L)$ for $L T A B>1)$. This parameter refers to a sink/source code name. Estimating a time-dependent generation rate requires providing index $L$ through command $>>>>$ INDEX.

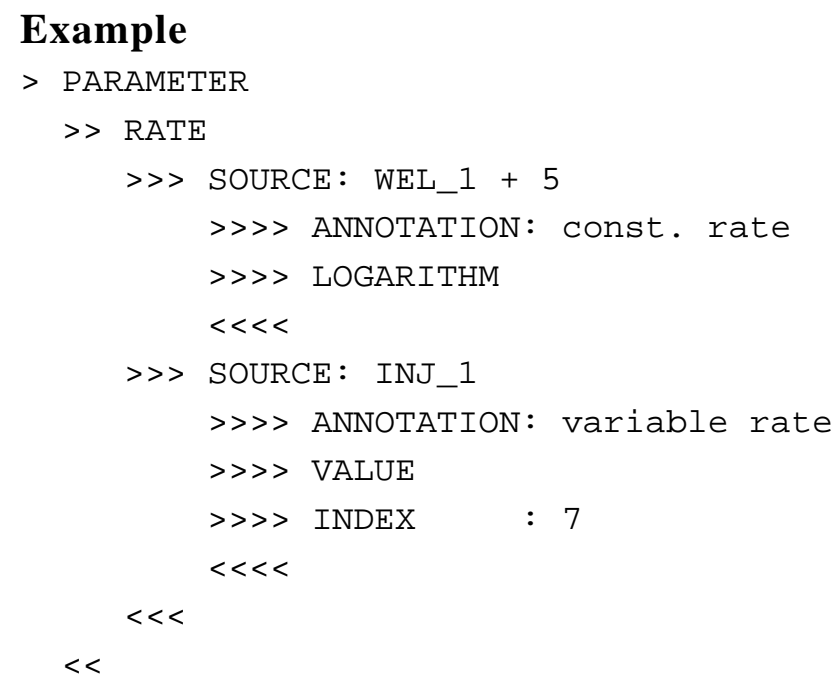

See Also

$>$ TIME (p) 


\section{Command}

>>> REDUCTION: max_red

\section{Parent Command}

>> CONVERGE

\section{Subcommand}

$-$

\section{Description}

By default, a TOUGH2 simulation stops if 20 consecutive time step reductions have occurred without convergence. This command changes the maximum number of allowable time step reductions to max_red.

Time step reductions occur if:

(1) the initial time step is too large (see TOUGH2 variable DELTEN or DLT (1), and command >> ADJUST),

(2) TOUGH2 parameter NOITE is too small,

(3) a failure in the EOS module occurs,

(4) boundary conditions are changed drastically during the course of the simulation.

Variable max_red should only be increased to address problem (4), i.e., when drastic changes in generation rates (TOUGH2 block GENER) are imposed, or if Dirichlet boundary conditions are changed using command $>>$ RESTART TIME or through subroutine USERBC.

\section{Example}

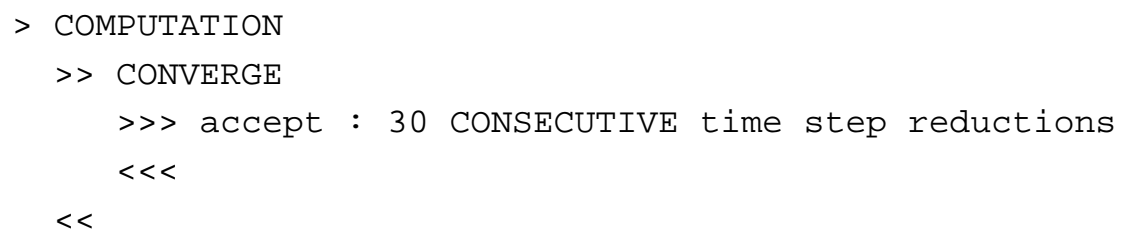

See Also

$>$ RESTART TIME, $>>$ ADJUST, $>>$ CONSECUTIVE 


\section{Command}

>> RELATIVE

\section{Parent Command}

$>$ PARAMETER

\section{Subcommand}

$>>>$ DEFAULT

$>>$ MATERIAL

\section{Description}

This command selects a parameter of the relative permeability function (TOUGH2 variable $R P(I P A R, N M A T)$ ) of a certain rock type, or a parameter of the default relative permeability function (TOUGH2 variable RPD (IPAR)). Command >>> INDEX must be used to select the parameter index IPAR. The physical meaning of the parameter depends on the type of relative permeability function selected in the TOUGH2 input file, variable IRP or IRPD. The admissible range should be specified explicitly to comply with parameter restrictions (see Pruess [1987], Appendix A).

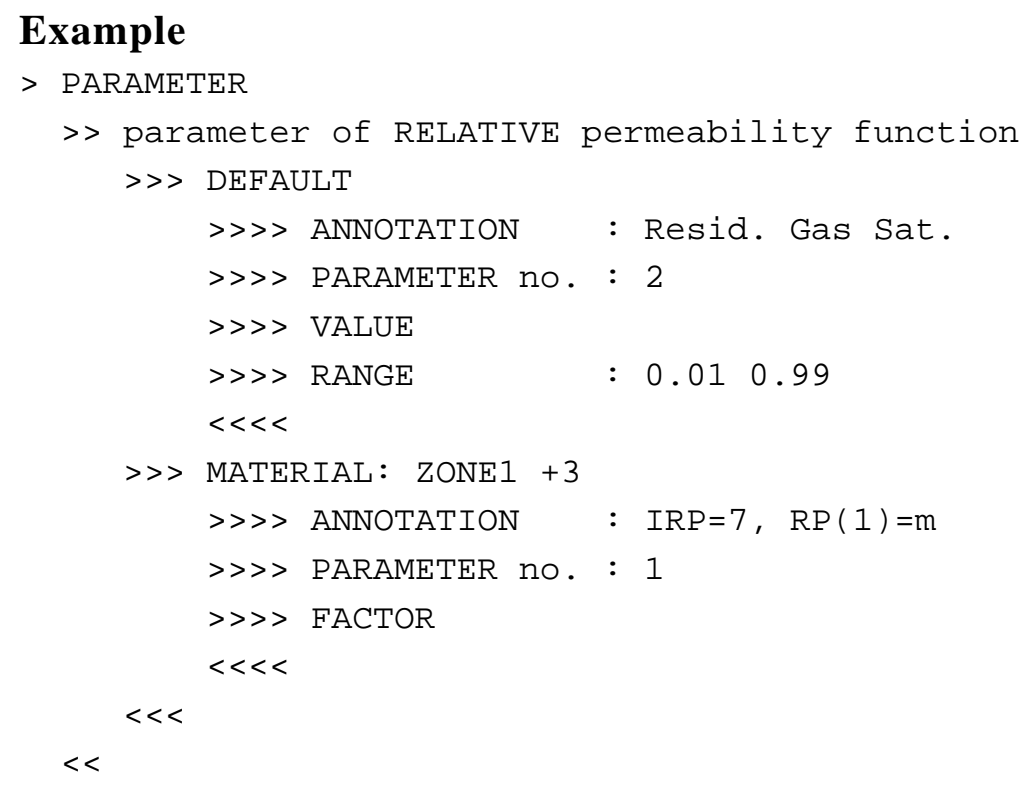

See Also

>> CAPILLARY 


\section{Command}

>>> RELATIVE: rel_err (\%)

\section{Parent Command}

all third-level commands in block > OBSERVATION

\section{Subcommand}

$-$

\section{Description}

This command takes a fraction of the observed value as the standard deviation of the measurement error (for more details see command $>>>>$ DEVIATION (o)). This option cannot be applied to data sets that contain zero measurements.

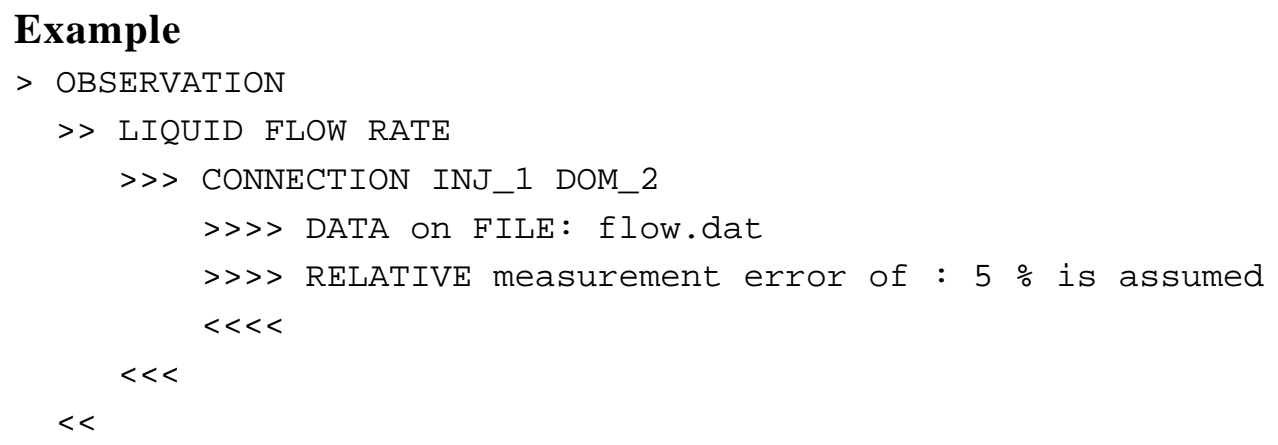

See Also

>>> DEVIATION (O) 


\section{Command}

$>>$ RESIDUAL

\section{Parent Command}

$>$ OUTPUT

\section{Subcommand}

$-$

\section{Description}

This command prints the observed and calculated system response as well as the residuals after each iTOUGH2 iteration. By default, the residuals are printed only once at the end of the optimization.

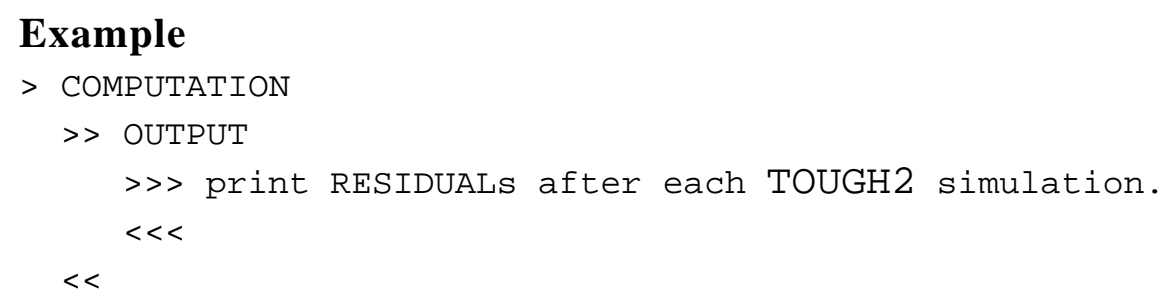

See Also

>> JACOBIAN 


\section{Command}

>> RESTART TIME: ntime (time_unit) (NEW)

\section{Parent Command}

$>$ OBSERVATION

\section{Subcommand}

\section{Description}

This command selects points in time at which the boundary conditions are changed by a step function, and reassigns primary variables and/or grid block volumes for selected elements. Such times are termed "restart times" because in standard TOUGH2 one would have to stop the simulation, and restart it after having changed the initial conditions of certain elements. This option is useful for modeling well tests, where the condition in the borehole is changed at certain points in time. For the simultaneous calibration of well tests consisting of many test events, it is imperative to model the entire sequence in a single simulation run, i.e. individual events must be connected. Recall, that complicated, time-varying boundary conditions can also be programmed into subroutine USERBC.

The specification of a restart time is identical to specifying calibration points (see command >> TIME (0)). Instead of calibration times, a restart time restart_time is indicated. Following the line containing the restart time, a list of element names elem (or element numbers) can be given, followed by an integer ipv indicating which primary variable is to be changed. If ipv is zero, the volume of the corresponding element is changed. Finally, the new primary variable or grid block volume, respectively, is given. The general format is as follows:

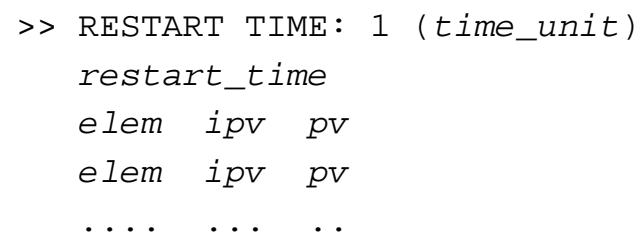

A comprehensive example is given below.

This option can also be used to periodically generate a SAVE file. This may be desirable for very long TOUGH2 simulations. In case of a computer failure, the run can be restarted at the time of the last restart time.

If keyword NEW is present, a new SAVE file with unique file name is created for each RESTART time.

\section{Example}

This example demonstrates the use of restart times to connect four individual test events into a single simulation. The test sequence starts with a shut-in recovery period, i.e. in the TOUGH2 input file the actual interval volume is assigned to the element representing the borehole. At time $t_{C P}$, a constant pressure pumping test is initiated, i.e., the element volume 
is increased to a very large number, and the prescribed interval pressure is specified as initial conditions. A shut-in pressure recovery period starts at time $t_{R E C}$, modeled by reassigning the actual interval volume. Finally, a pulse test is simulated, assuming that all the gas that potentially accumulates in the borehole interval has been released at time $t_{P U L S E}$, prior to applying a short pressure pulse. The pressure response in the injection well is schematically shown in the figure below, followed by the appropriate iTOUGH2 block with restart times and boundary condition specifications.
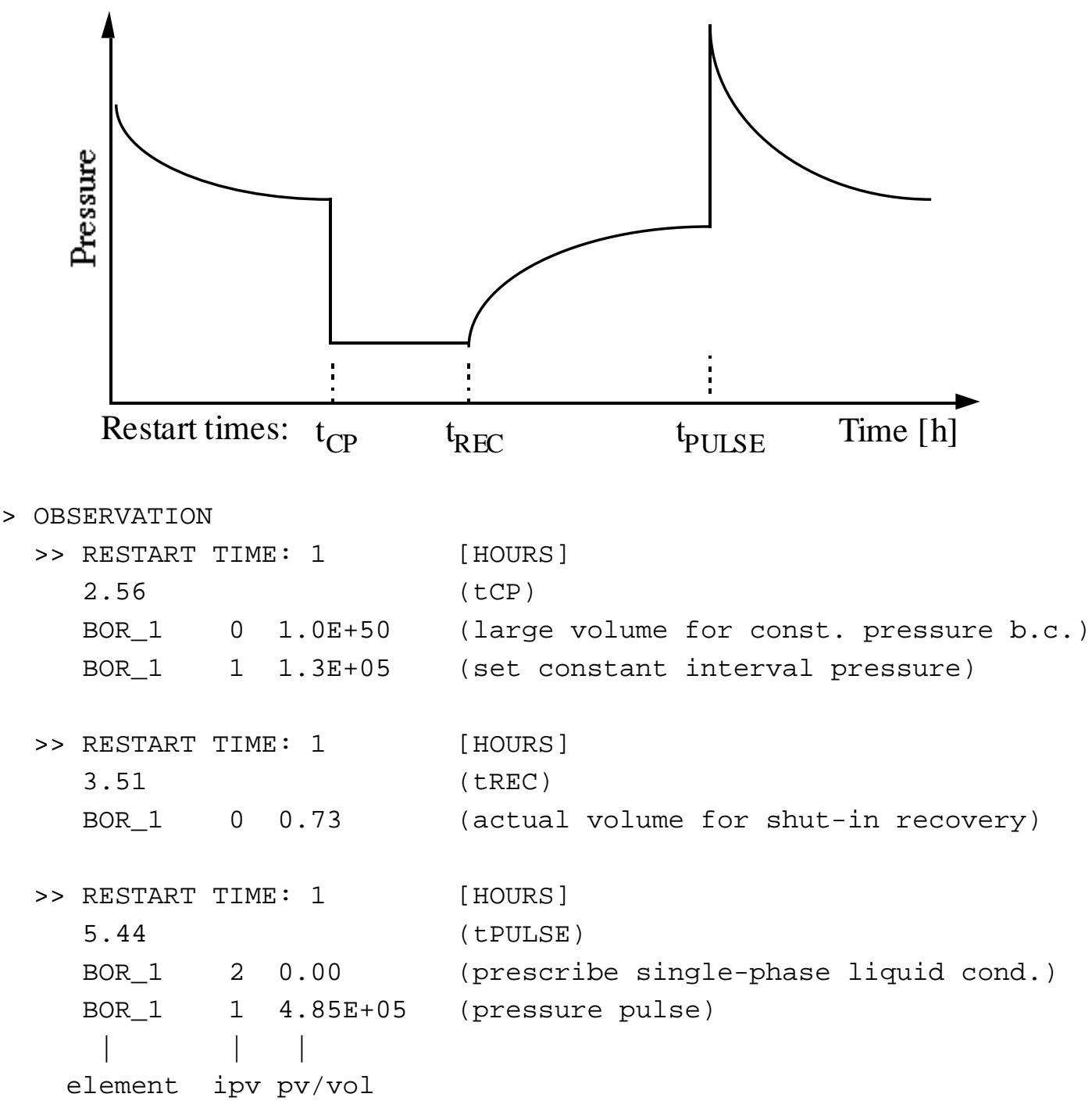

TOUGH2 stops at each restart time, writes a SAVE file, reads it as an INCON file, changes initial conditions, i.e. updates the primary variables or grid block volumes of the indicated elements, and continues the simulation.

See Also

$>$ TIME (O) 


\section{Command}

>>> ROCKS: mat_name (mat_name_i_...) (+ iplus)

\section{Parent Command}

all second-level commands in block $>$ PARAMETER referring to a material name

\section{Subcommand}

(synonym for command $>>$ MATERIAL)

\section{Description}

(see command >> MATERIAL)

\section{Example}

See Also

>> MATERIAL 


\section{Command}

>> SATURATION (phase_name/PHASE: iphase)

\section{Parent Command}

$>$ OBSERVATION

\section{Subcommand}

>> ELEMENT

\section{Description}

This command selects phase saturation as an observation type. This observation type refers to an element. The phase name phase_name or phase number iphase, which depend on the EOS module being used, are listed in the iTOUGH2 header. They can be specified either on the command line or using the subcommand $>>>>$ PHASE.

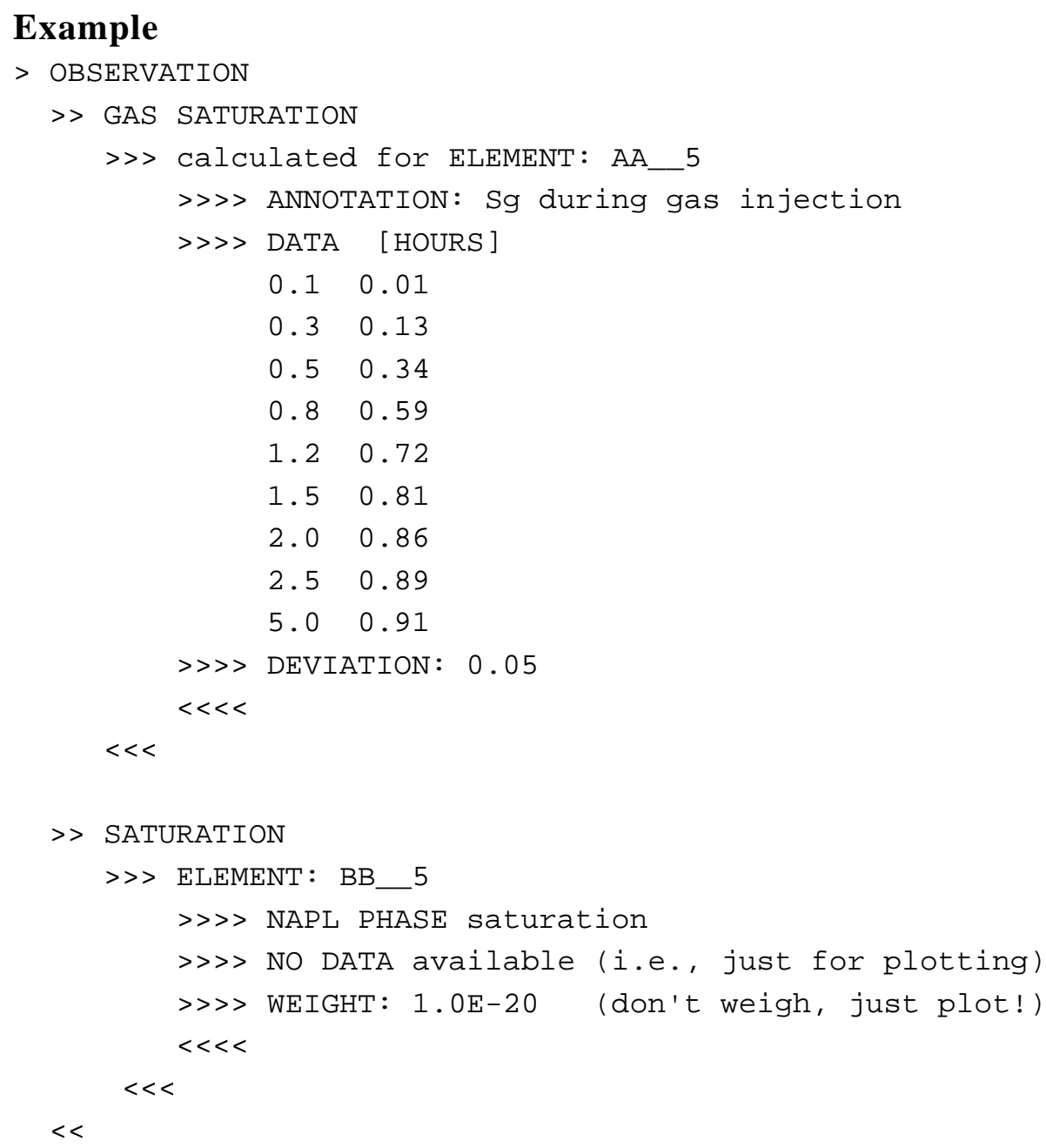

See Also

$-$ 


\section{Command}

$>$ SCALE

\section{Parent Command}

$>$ PARAMETER

\section{Subcommand}

$>>$ NONE

\section{Description}

This command selects as a parameter a grid scaling factor. Nodal distances, interface areas, and gridblock volumes will be scaled accordingly. There are three grid scaling factors, referring to the three directions specifed by TOUGH2 variable ISOT. The direction is selected through command $>>>>$ INDEX. If all three directions are selected, the mesh is scaled isotropically (see TOUGH2 variable SCALE).

Selecting a grid scaling factor as a parameter can be used to design and optimize the horizontal and/or vertical spacing between injection points.

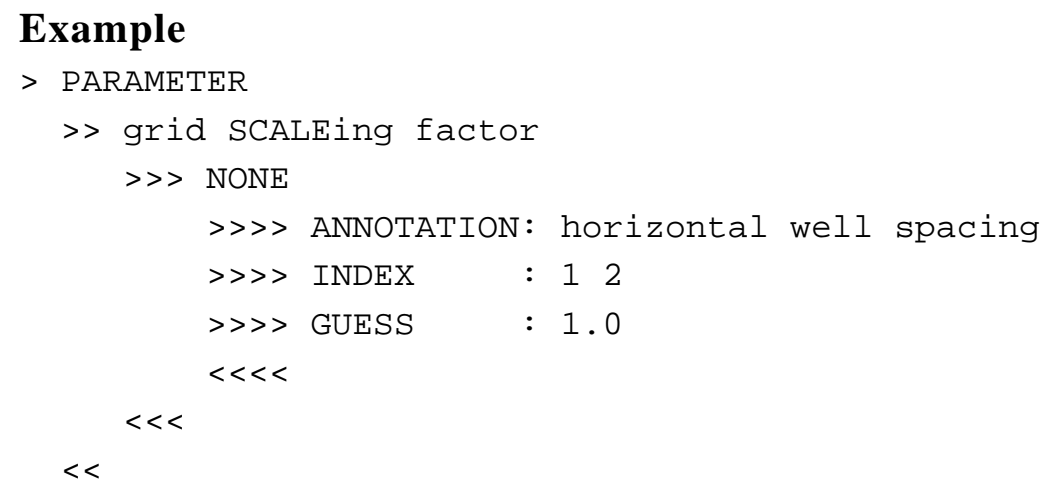

See Also

$-$ 


\section{Command}

>>> SCHEDULE: beta

\section{Parent Command}

$>>$ ANNEAL

\section{Subcommand}

$-$

\section{Description}

This command defines the annealing schedule for Simulated Annealing minimization. The annealing schedule is a function describing the temperature reduction (and thus the probability of accepting an uphill step, see command $>>>$ ANNEAL). The temperature is updated after a certain number of successful steps have been performed (see command $>>>$ STEP (a)).

There are two functions available. If beta is in the range $0<\beta<1$ (typically 0.9 ), the following annealing schedule is used:

$$
\tau_{k}=\beta^{k} \cdot \tau_{0}
$$

where $k$ is the iteration index, and $\tau_{0}$ is the initial temperature specified by command >>> TEMPERATURE. Ifbeta is greater than one, the following annealing schedule is invoked:

$$
\tau_{k}=\tau_{0}(1-k / K)^{\beta}
$$

where $K$ is the maximum number of iterations (see command $>>>$ ITERATION (a)).

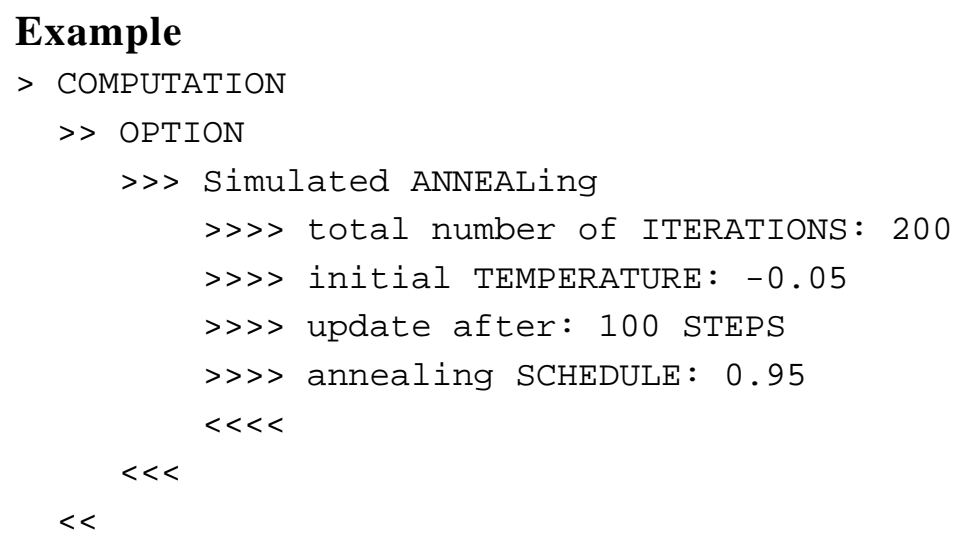

See Also

>>> ITERATION (a), >>> STEP, >>> TEMPERATURE 


\section{Command}

>> SECONDARY (phase_name/PHASE: iphase) (: ipar)

\section{Parent Command}

$>$ OBSERVATION

\section{Subcommand}

$>>$ ELEMENT

\section{Description}

This command selects thermophysical properties, which are so-called "secondary parameters" in TOUGH2, as an observation type. This observation type refers to an element. The secondary parameter refers to elements of TOUGH2 vector PAR (see Pruess [1991a], Figure 2). The index ipar must be provided in the command line after a colon or through the fourth-level command >>> INDEX. The fluid phase must also be identified. The phase name phase_name or phase number iphase, which depend on the EOS module being used, are listed in the iTOUGH2 header. They can be specified either on the command line or using the fourth-level command $>>>>$ PHASE.

The following table lists the thermophysical property addressed by ipar. Some of the properties can be selected using an alternative second-level command.

\begin{tabular}{cll}
\hline ipar & Secondary Parameter & Alternative Command \\
\hline 1 & phase saturation & SATURATION \\
2 & relative permeability & - \\
3 & viscosity & - \\
4 & density & - \\
5 & specific enthalpy & - \\
6 & capillary pressure & PRESSURE \\
$N B+i$ & mass fraction of component $i$ & MASS FRACTION \\
\hline
\end{tabular}

\section{Example}

$>$ OBSERVATION

>> SECONDARY parameter No: 3

>> ELEMENT: AA_5

>>> ANNOTATION: gas viscosity

$>>>$ GAS PHASE

$>>>$ NO DATA

$<<<<$

$<<<$

See Also

$>>$ MASS FRACTION, >> PRESSURE, >> SATURATION,

$>>>$ PHASE, $>>>>$ INDEX 


\section{Command}

$>$ SELEC

\section{Parent Command}

$>$ PARAMETER

\section{Subcommand}

$>>$ MODEL

\section{Description}

This command selects as a parameter one of the SELEC parameters (TOUGH2 variable $F E(I))$. The physical meaning of the parameter depends on the module being used. The index I must be provided through command $>>>>$ INDEX.

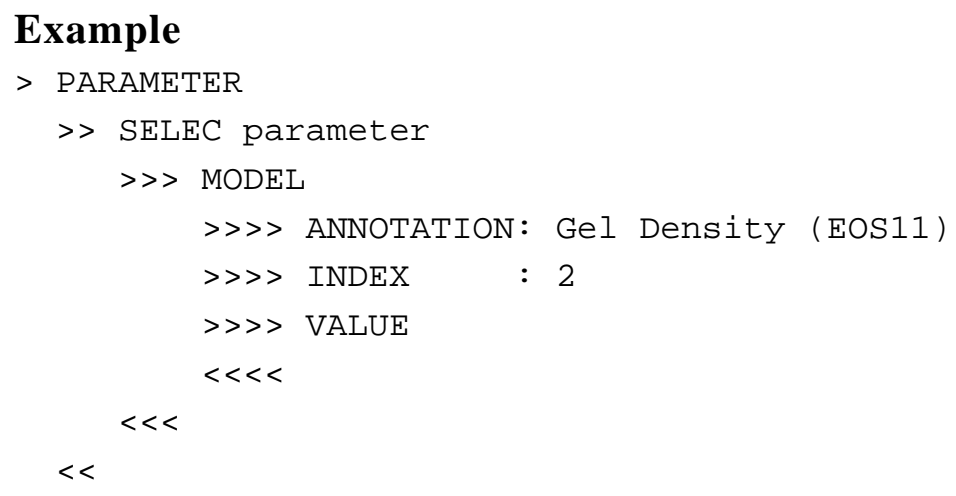

See Also 


\section{Command}

$>>$ SELECT

Parent Command

$>>$ OPTION

\section{Subcommand}

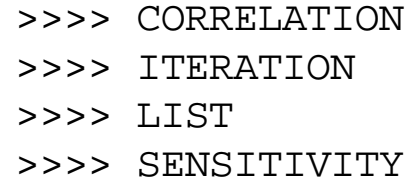

\section{Description}

This command invokes the automatic parameter selection option of iTOUGH2. The parameters defined in block $>$ PARAMETER are screened according to two selection criteria (see commands $>>>>$ SENSITIVITY and >>> CORRELATION). Only the most sensitive and/or most independent parameters are subjected to the optimization process. Automatic parameter selection allows one to submit a larger set of parameters to the estimation process. If a parameter is not sensitive enough to be estimated from the available data, it is automatically removed from the set of parameters being updated. This makes the inversion faster because fewer parameters must be perturbed for calculating the Jacobian matrix (the full Jacobian is only calculated every few iteration when the selection criteria are reevaluated). The inversion is also more stable. Parameters that are not sensitive or highly correlated tend to be changed drastically during an iTOUGH2 iteration which may cause unnecessary numerical difficulties. If they are (temporarily) removed from the parameter set, they remain at their initial or current value.

An additional advantage of using this option is the fact that sensitivities, estimation uncertainties, and parameter correlations are calculated for all the specified parameters, regardless of whether they are updated during the optimization.

Due to the non-linearity of the inverse problem at hand, sensitivity coefficients and parameter correlations constantly change during the optimization. Therefore, the selection criteria must be reevaluated from time to time (see command $>>>>$ ITERATION (s)), i.e., parameters may be deactivated and reactivated during the course of an inversion.

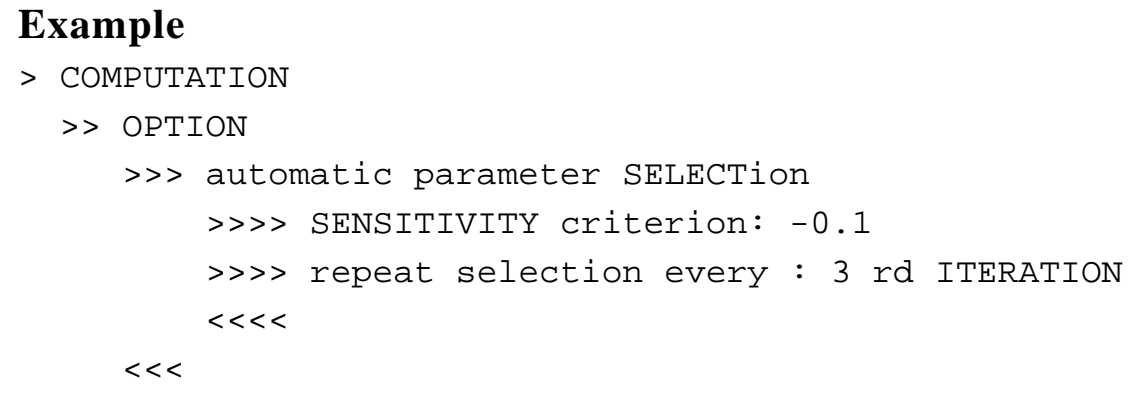

See Also 


\section{Command}

$>>$ SENSITIVITY

or

$>>$ DESIGN

Parent Command

$>$ OPTION

\section{Subcommand}

\section{Description}

This command makes iTOUGH2 evaluate the sensitivity matrix without performing any optimization. By default, the scaled Jacobian matrix, i.e., the sensitivity coefficients scaled by the standard deviation of the observation and expected parameter variation, respectively, is printed to the iTOUGH2 output file:

$$
\tilde{J}_{i j}=J_{i j} \frac{\sigma_{p_{j}}}{\sigma_{z_{i}}}=\frac{\partial z_{i}}{\partial p_{j}} \cdot \frac{\sigma_{p_{j}}}{\sigma_{z_{i}}}
$$

In addition, the unscaled sensitivity coefficients can be printed by invoking subcommand >> SENSITIVITY in block >> OUTPUT.

This information can be used to identify the parameters that most strongly affect the system behavior at actual or potential observation points. Similarly, the relative information content of actual or potential observations, i.e., the contribution of each data point to the solution of the inverse problem can be evaluated.

Based on this command, iTOUGH2 also calculates the covariance matrix of the estimated parameters, i.e., the estimation uncertainty under the assumption that the variances of the residuals are accurately depicted by the prior covariance matrix $\mathbf{C}_{z z}$. This information along with the global sensitivity measures (sums of absolute sensitivity coefficients) can be used to optimize the design of an experiment.

It is recommended to use a relatively large perturbation factor (see command >> PERTURB), possibly in combination with centered finite difference quotients (see command $>>>$ CENTERED) for the purpose of sensitivity analysis.

\section{Example}

$>$ COMPUTATION

$>>$ OPTION

$>>$ perform a SENSITIVITY analysis for test DESIGN

$<<<$

$<<$

See Also

>> CENTERED, >> PERTURB, >> SENSITIVITY (ou) 


\section{Command}

>> SENSITIVITY

\section{Parent Command}

>> OUTPUT

\section{Subcommand}

-

\section{Description}

This command prints the sensitivity matrix to the iTOUGH2 output file. The elements of the sensitivity matrix are the partial derivatives of the system response at the observation points with respect to the parameters:

$$
J_{i j}=\frac{\partial z_{i}}{\partial p_{j}}
$$

By default, iTOUGH2 prints the scaled Jacobian matrix, the elements of which are the sensitivity coefficients scaled by the ratio of the standard deviation of the observation and expected parameter variation:

$$
\tilde{J}_{i j}=J_{i j} \frac{\sigma_{p_{j}}}{\sigma_{z_{i}}}=\frac{\partial z_{i}}{\partial p_{j}} \cdot \frac{\sigma_{p_{j}}}{\sigma_{z_{i}}}
$$

\section{Example}

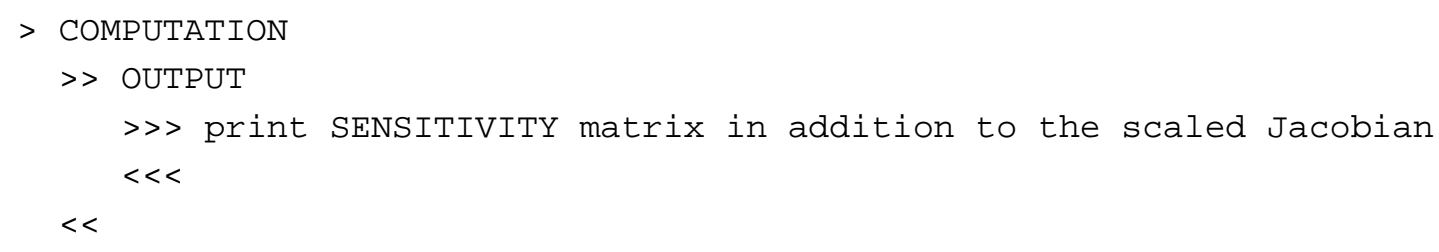

\section{See Also}




\section{Command}

>>> SENSITIVITY: (-) rsens

\section{Parent Command}

$>>$ SELECT

\section{Subcommand}

$-$

\section{Description}

This command defines one of the criteria used for automatic parameter selection. It examines the potential of a parameter to reduce the objective function:

$$
\delta=|\partial S|
$$

Here, $\partial S$ is the change of the objective function if the parameter is perturbed by a certain, small value (see command $>>>$ PERTURB).

Normalizing to the maximum value $\delta_{\max }$ yields the selection criterion $\omega$ :

$$
\omega=\frac{\delta}{\delta_{\max }} \quad 0<\omega \leq 1
$$

Those parameters with an $\omega$-value larger than $\mid$ rsens $\mid$, i.e., the most sensitive parameters, are selected. Parameters which are unlikely to significantly reduce the objective function are (temporarily) excluded from the optimization process.

If a negative value is given for rsens, the selection criterion is relaxed with each iteration $k$, and reaches zero for the last iteration max_iter, i.e., all parameters specified in block $>$ PARAMETER are selected for the final step.

$$
\text { rsens }_{k}=|r \operatorname{sens}| \cdot\left(1-\frac{k}{\text { max_iter }_{-}}\right)
$$

\section{Example}

$>$ COMPUTATION

$>>$ OPTION

>> SELECT parameter automatically every

>>> : 3 ITERATIONS

$>>>$ based on the SENSITIVITY criterion with rsens : -0.10

$<<<<$

$<<<$

$<<$

See Also

>>> ITERATION (s), >>> CORRELATION 


\section{Command}

>> SET: iset

\section{Parent Command}

$>>$ DRIFT

$>$ FACTOR

$>$ LAG

$>$ SHIFT

\section{Subcommand}

all fourth-level commands in block > PARAMETER

\section{Description}

The parent command of this command identifies a parameter that refers to a data set, i.e., it manipulates the observed data. The data set is identified by its ordering number iset as specified in block > OBSERVATION. Alternatively, iset can be read using subcommand >>> INDEX; the latter command must be used if multiple sets are selected.

\section{Example}

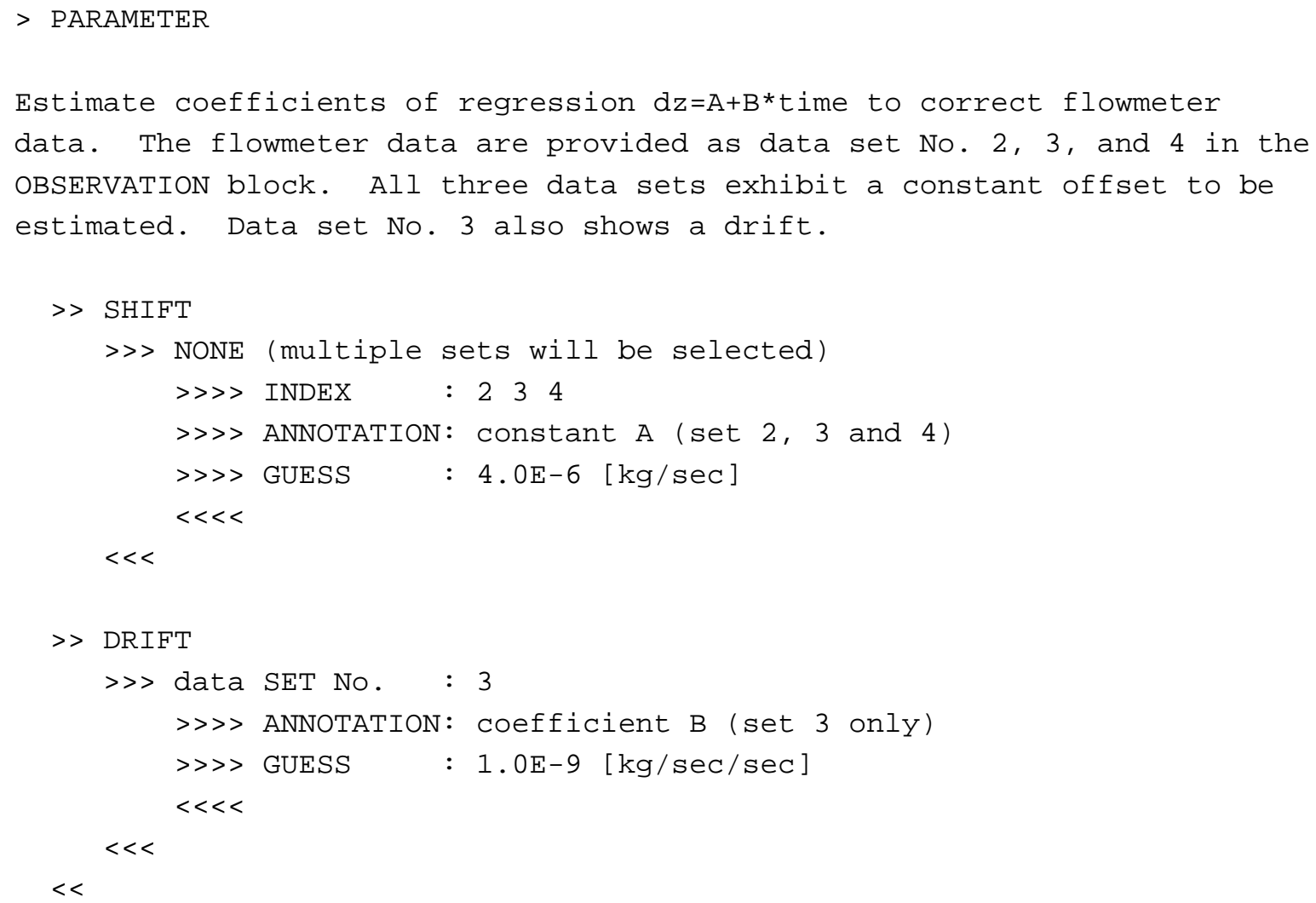

See Also

- 


\section{Command}

>>> SET: iset

\section{Parent Command}

all third-level commands in block > OBSERVATION

\section{Subcommand}

\section{Description}

Multiple data sets can be stored on a single file, separated by a single line containing characters. This command is used to select the iset'th data set on a data file. By default, data are read from the first set following the header lines (see command $>>>>$ HEADER).

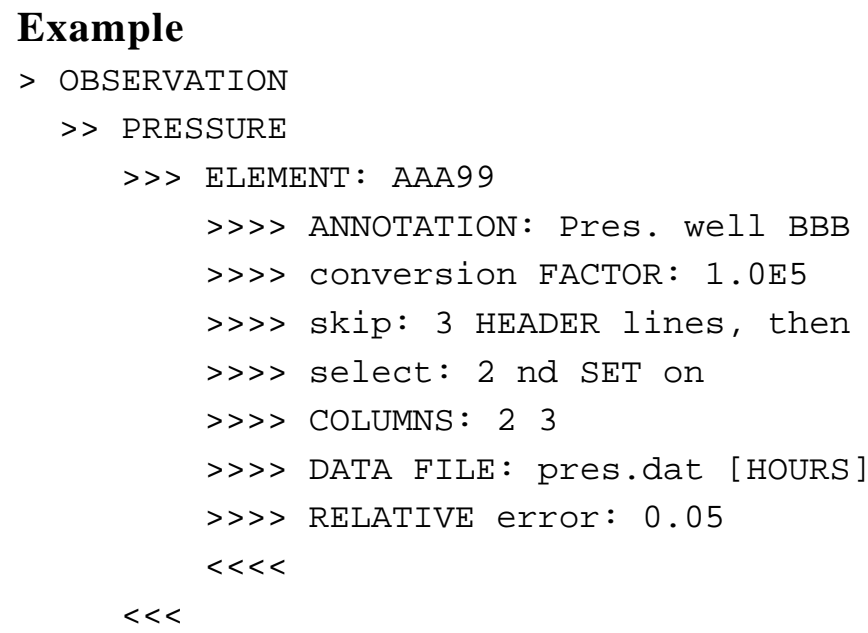

The content of file pres.dat is:

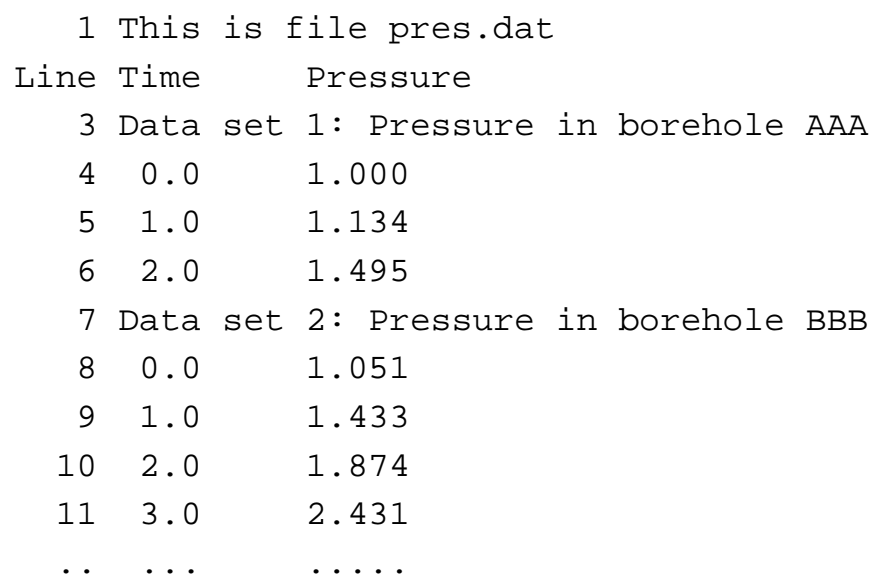

See Also

>>> HEADER 


\section{Command}

$>$ SHIFT

\section{Parent Command}

$>$ PARAMETER

\section{Subcommand}

$>>$ NONE

$>>\mathrm{SET}$

\section{Description}

This command selects as a parameter a constant used to shift the calculated TOUGH2 output. The constant is added to the output that refers to a specific data set:

$$
z=z_{\text {TOUGH2 } 2}+\text { shift }
$$

where shift is the constant added to the calculated TOUGH2 output $z_{\text {TOUGH2 }}$. The result $z$ is compared to the measurement $z^{*}$ of the corresponding data set.

This option allows removal of a constant from the data. For example, if matching relative pressure data that fluctuate around an unknown mean, or if a flowmeter exhibits an unknown offset that needs to be estimated, the shift parameter being estimated is the mean or offset, respectively.

A non-zero value must be provided as an initial guess through the iTOUGH2 input file using command $>>>>$ GUESS.

The data set is identified by number using command $>>>\operatorname{SET} \quad(\mathrm{p})$ or command $>>>\operatorname{INDEX}(\mathrm{p})$.

If the constant is known, i.e. does not need to be estimated, use command $>>>>$ SHIFT, a subcommand of > OBSERVATION, to shift the data.

\section{Example}

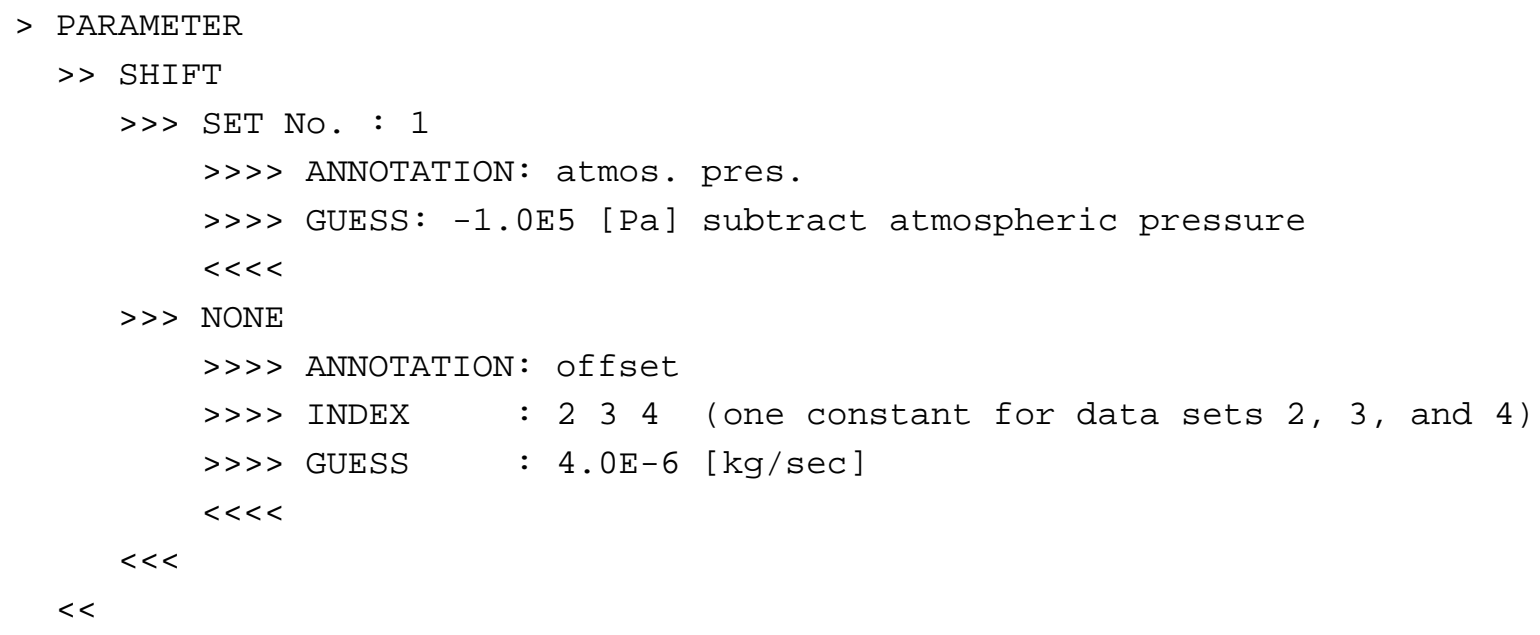

See Also

> DRIFT, > FACTOR, > LAG, >> SET (p), >>> SHIFT 


\section{Command}

>>> SHIFT: shift (TIME (time_unit))

\section{Parent Command}

all third-level commands in block > OBSERVATION

\section{Subcommand}

\section{Description}

This command shifts data values or observation times by a known, constant value shift. The effect of this command is a direct data manipulation which must be distinguished from estimating an unknown constant that shifts the model output (see command $>$ SHIFT). If keyword TIME is present on the command line, the constant shift is added to all observation times of the corresponding data set. This is useful to adjust the reference time of the data record if it is different from the starting time of the simulation (TOUGH2 variable TSTART). The time unit can be selected using one of the time_unit keywords.

If keyword TIME is not present, shift is added to all observed values. The units of shift must match those of the observed data (see command $>>>>$ FACTOR).

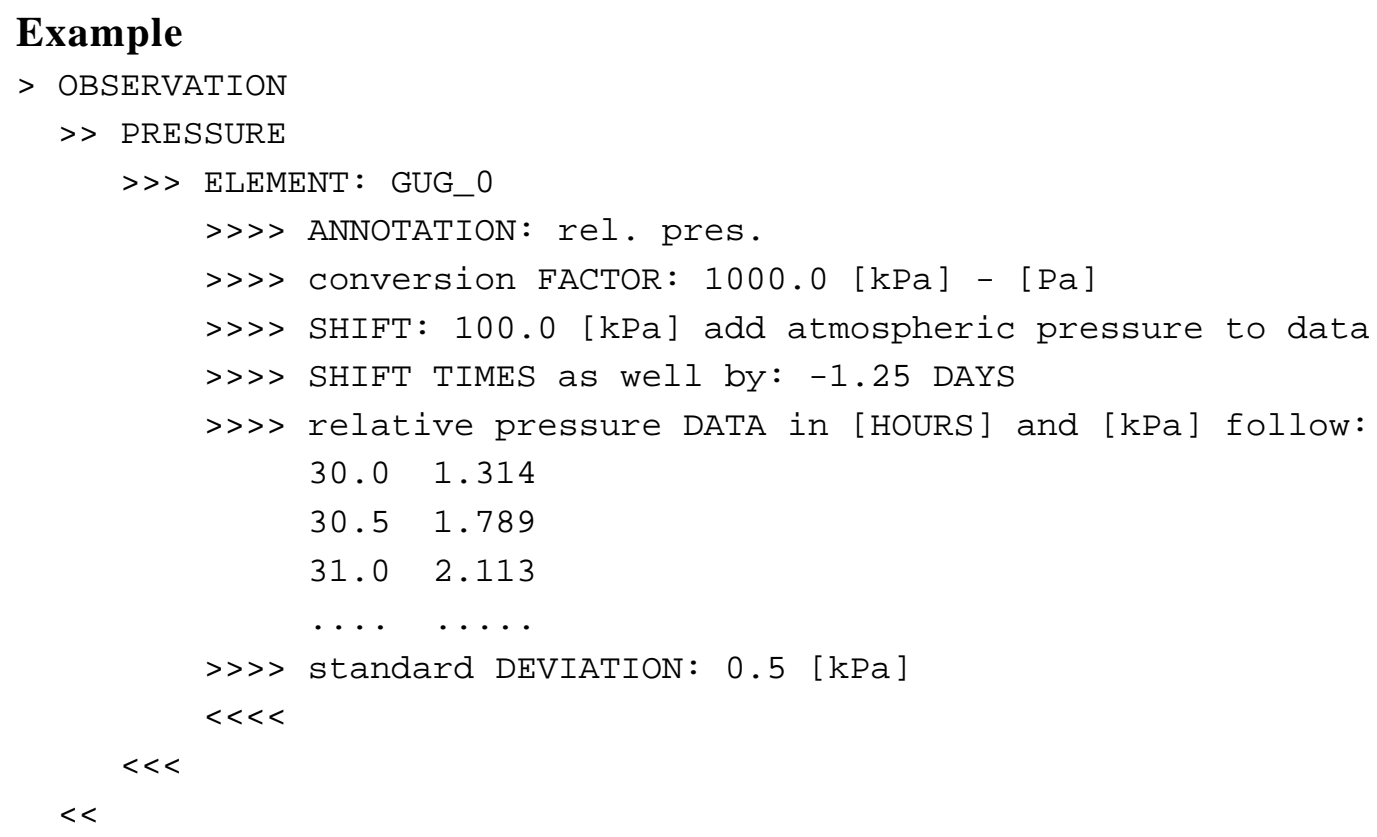




\section{Command}

>> SIMPLEX

\section{Parent Command}

$>$ OPTION

\section{Subcommand}

$-$

\section{Description}

This command selects the downhill simplex method to minimize the objective function. The downhill simplex method does not calculate derivatives and can therefore be used for discontinuous objective functions. The initial simplex is defined by the initial parameter set $\mathbf{p}_{0}$ and $n$ additional points given by :

$$
\mathbf{p}_{i}=\mathbf{p}_{0}+\sigma_{i} \cdot \mathbf{e}_{i} \quad i=2, \ldots,(n+1)
$$

where $\sigma_{i}$ is the parameter variation given by commands $>>>>$ VARIATION or $>>>$ DEVIATION (p), and the $\mathbf{e}_{i}$ 's are $n$ unit vectors along the parameter axis. At the end of minimization by means of the simplex algorithm, the Jacobian matrix is evaluated to allow for a standard error analysis.

The simplex algorithm is most useful in connection with the $\mathrm{L}_{1}$-estimator and discontinuous cost functions.

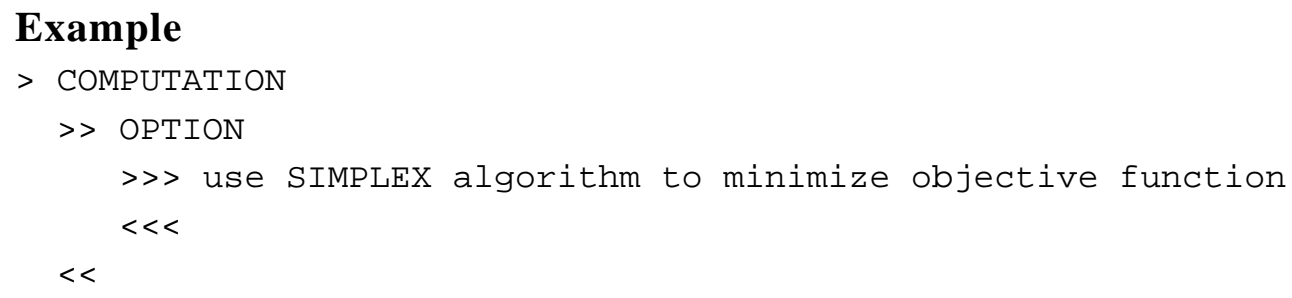

See Also

>> ANNEAL, >> GAUSS-NEWTON, >> GRID SEARCH, $>>>$ LEVENBERG-MARQUARDT 


\section{Command}

>> SIMULATION: mtough2

\section{Parent Command}

>> CONVERGE

\section{Subcommand}

$-$

\section{Description}

This command limits the number of TOUGH2 simulations performed during an inversion. A rough estimate of the total number of TOUGH2 simulations during an inversion can be made given the number of iterations requested (see command $>>>$ ITERATION) and the type of finite difference quotients calculated (see commands $>>>$ FORWARD and $>>>$ CENTERED). However, there is an unknown number of unsuccessful steps that cannot be accounted for.

The maximum number of TOUGH2 simulations may also be limited in order to stop and examine a specific simulation that leads to convergence problems (see command >> INCOMPLETE).

If performing Monte Carlo simulations (see command $>>>$ MONTE CARLO), mtough2 is the number of parameter sets generated and thus the number of realizations of the calculated system output.

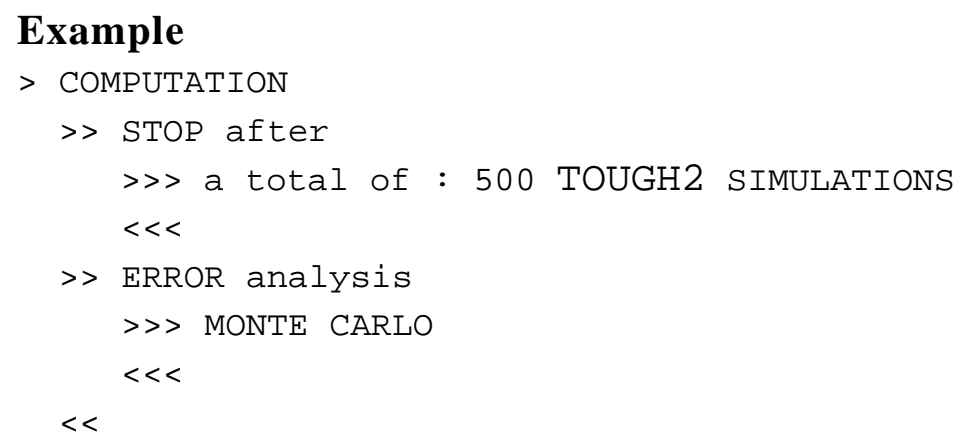

See Also

>> ITERATION, >> MONTE CARLO, >>> INCOMPLETE 
Command

>> SINK: sink_name (sink_name_i ...) (+ iplus)

\section{Parent Command}

all second-level commands in block > PARAMETER or $>$ OBSERVATION referring to sink names

\section{Subcommand}

all fourth-level commands in block > PARAMETER or $>$ OBSERVATION

\section{Description}

(synonym for command $>>$ SOURCE)

\section{Example}

(see command >> SOURCE)

See Also

>> SOURCE 


\section{Command}

$>$ SKIN

\section{Parent Command}

$>$ PARAMETER

\section{Subcommand}

>> MATERIAL

\section{Description}

This command selects as a parameter the skin zone radius around a well. In general, the radial thickness of the skin zone is predefined by the discretization of the flow region. In order to estimate the skin zone radius, the TOUGH2 mesh must be generated using the MESHMAKER utility. Furthermore, two zones must be generated using logarithmically increasing radial distances (option LOGAR). The skin radius is defined by the parameter RLOG after the first appearance of keyword LOGAR. In addition, an I5 integer must be provided after NRAD, NEQU, and NLOG, indicating the material type number for the corresponding grid blocks (see example below). Estimation of a skin zone radius may be unstable if changing the discretization significantly affects the modeling results.

\section{Example}

The following MESHMAKER block is from a TOUGH2 input file. A radial mesh is generated with a borehole of radius $0.1 \mathrm{~m}$ (material type 1), a skin zone of material type 2 and initial radius of $0.3 \mathrm{~m}$, partitioned into 20 grid blocks, and an outer zone of material type 3 which extends to a radial distance of $10.0 \mathrm{~m}$. The thickness of the layer is $1.0 \mathrm{~m}$. The skin zone radius to be estimated is printed in italics.

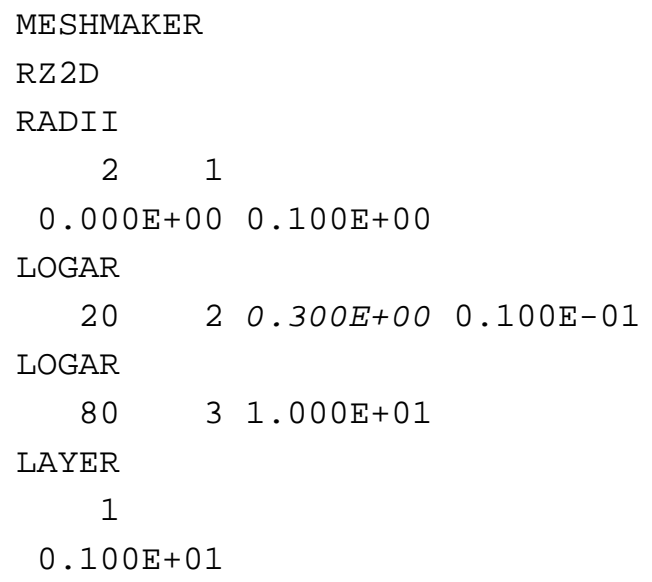


The corresponding parameter block in the iTOUGH2 input file reads:

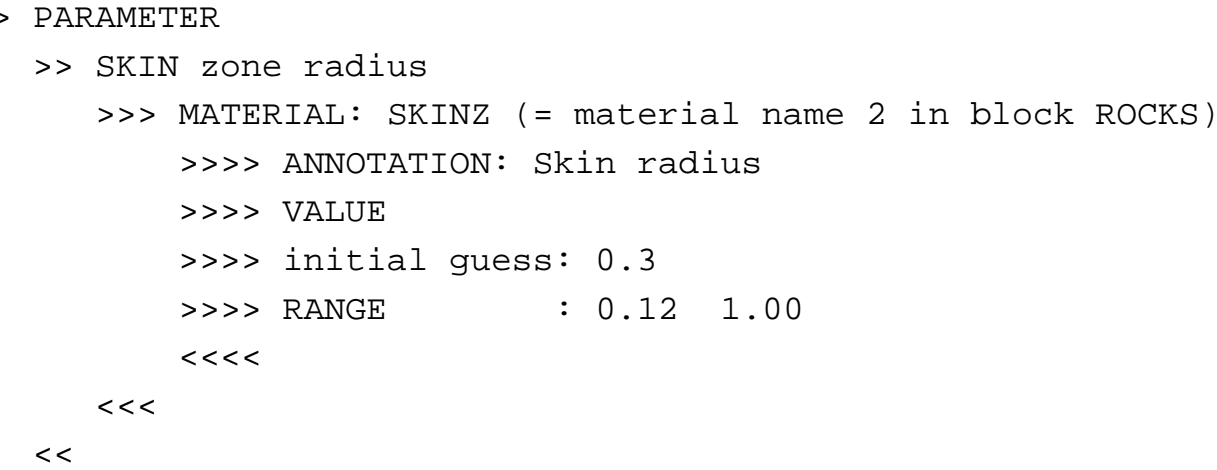

See Also

$-$ 


\section{Command}

>>> SKIP: nskip

\section{Parent Command}

all third-level commands in block > OBSERVATION

\section{Subcommand}

Description

(synonym for command $>>>>$ HEADER)

\section{Example}

(see command $>>>>$ HEADER)

\section{See Also}

$>>>$ HEADER 


\section{Command}

>> SOURCE: source_name (source_name_i ...) (+ iplus)

or

>> SINK: sink_name (sink_name_i ...) (+ iplus)

\section{Parent Command}

all second-level commands in block > PARAMETER or > OBSERVATION referring to source names.

\section{Subcommand}

all fourth-level commands in block > PARAMETER or > OBSERVATION, respectively

\section{Description}

This command reads one or more sink/source code names (TOUGH2 variable SOURCE) or element names associated with a sink or source (TOUGH2 variable $E L E G)$ as specified in the TOUGH2 block GENER. Both parameter and observations may refer to sources. Sources and source elements are designated by a three-character/two-integer (FORTRAN format: AAAII) code name. Blanks in the names as printed in the TOUGH2 output file must be replaced by underscores (e.g. a source or element name specified in the TOUGH2 input file as 'B 007 ' is printed as 'B $0 \quad 7$ ' in the TOUGH2 output file. Therefore, it must be addressed in the iTOUGH2 input file as 'B_0_7').

Multiple names can be specified. A sequence of iplus elements can be generated by increasing the number of the last element. The following two command lines are identical:

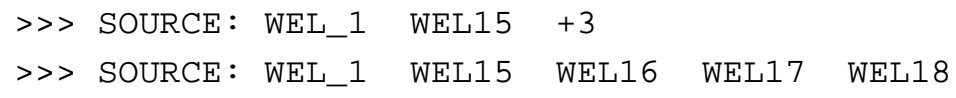

\section{Example}

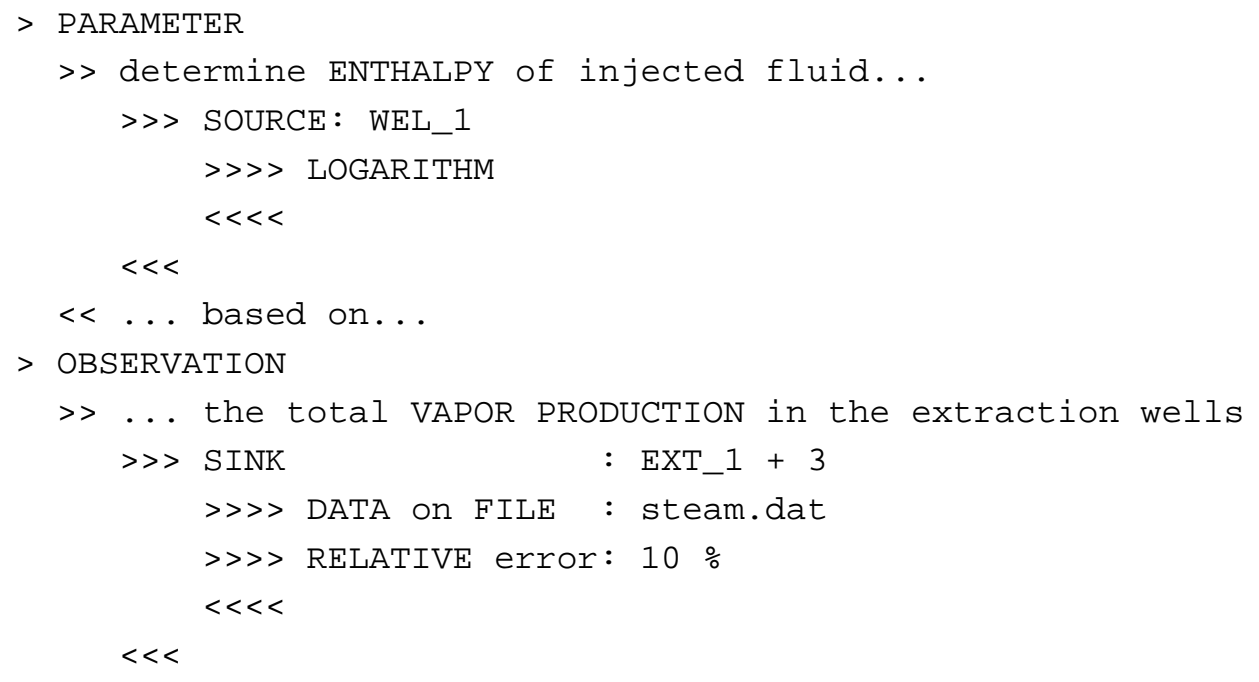

See Also

- 


\section{Command}

>> STEADY-STATE (SAVE) (: max_time_step)

\section{Parent Command}

>> OPTION

\section{Subcommand}

\section{Description}

This command allows TOUGH2 to reach steady state prior to or after a transient simulation. This option can be used to calibrate against steady-state data (only or in combination with transient data), or to reach an equilibrium as initial conditions for a subsequent transient simulation.

Note that a transient TOUGH2 simulation must be performed to reach steady state. Steady state is usually indicated by one of the following convergence failures:

- $\quad$ too many time steps converged on a single Newton-Raphson iteration (see command >>> CONSECUTIVE);

- convergence failure following two time steps that converged on a single NewtonRaphson iteration;

- $\quad$ too many time step reductions (see command >>> REDUCTION);

- $\quad$ maximum number of time steps reached (TOUGH2 variable $M C Y C$ );

- maximum simulation time reached (TOUGH2 variable TIMAX);

- $\quad$ maximum time step size reached (use a colon on the command line followed by max_time_step to select a maximum time step size. The max_time_step must be smaller than TOUGH2 variable DELTMX).

iTOUGH2 usually stops if a TOUGH2 run terminates due to one of the above mentioned convergence failures (see command $>>$ INCOMPLETE). If command $>>>$ STEADYSTATE is present, however, it accepts convergence failures and proceeds with the inversion.

Inversions involving steady-state runs can be greatly accelerated by using keyword SAVE on the command line. After the first steady-state run has been completed, iTOUGH2 takes the steady-state conditions stored on file $S A V E$ as the initial condition (file INCON) for the next TOUGH2 run, sets the initial time step to the (usually large) last time step of the previous simulation, and updates the parameter set. Since the parameter set is changed only slightly between subsequent TOUGH2 simulations, the new steady state is usually reached within a few additional time steps. This option should not be used if also calibrating against transient data preceding the steady-state data point.

Figure (a) below illustrates the use of the steady-state option assuming that calibration against steady-state data is to be performed. A steady-state calibration time $t_{\infty}$ should be specified at a very late point in time (i.e., beyond the transient phase of the simulation), and the same time should be assigned to the observed steady-state data point in block >>> DATA. (In addition to the steady-state point, one might also specify calibration points during the 
transient phase). The TOUGH2 simulation proceeds until a convergence failure occurs at an unknown point in time $t_{c f}$. The primary variables at that point are written to file $S A V E$ and reused as initial conditions for the subsequent TOUGH2 run (only if keyword SAVE is present). Then, the calculated system state at time $t_{c f}$ is compared to the steady-state data point at time $t_{\infty}$ (make sure that $t_{\infty}$ is always greater than $t_{c f}$, e.g., set $t_{\infty}=1 \mathrm{E} 20$ ).

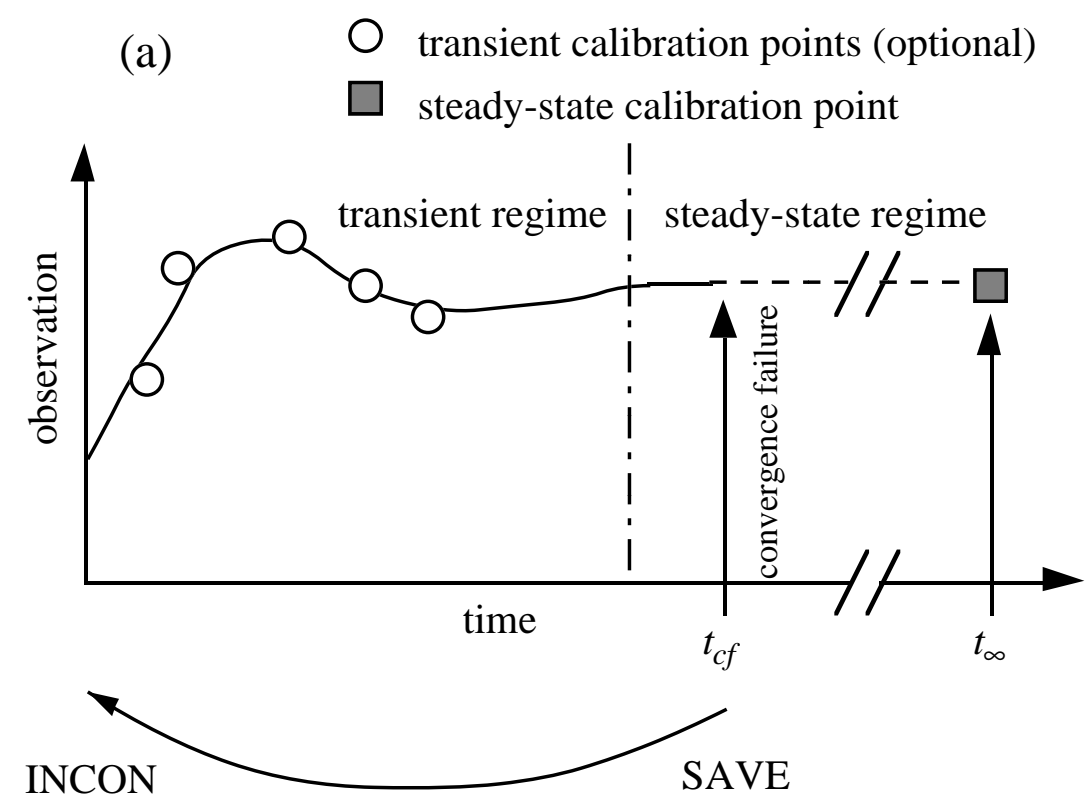

(b)

$$
\begin{aligned}
& \bigcirc \text { transient calibration points } \\
& \square \quad \text { steady-state calibration point (optional) }
\end{aligned}
$$

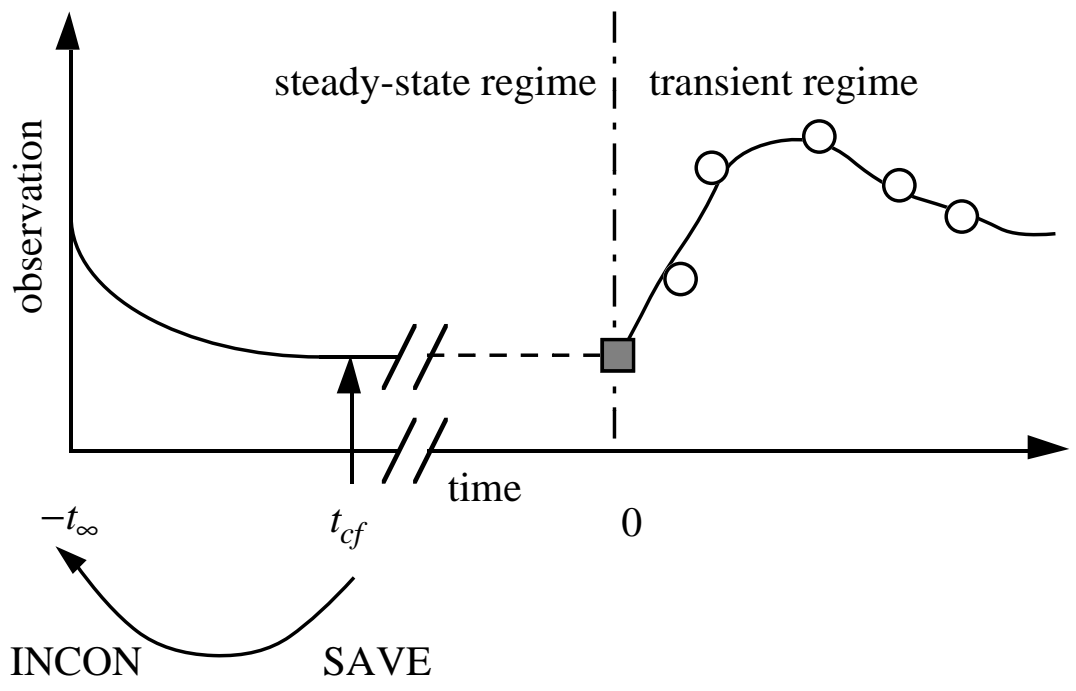


The second application of the steady-state option is illustrated in Figure (b). If a steady-state regime is to precede a transient regime within a single simulation (e.g., to assure initial conditions are at equilibrium, a state that depends on the parameters to be estimated), a negative starting time $-t_{\infty}$ (TOUGH2 variable TSTART) must be specified. Ensure that $\left|-t_{\infty}\right|$ is larger than the duration required to reach steady state, e.g., $-t_{\infty}=-1 \mathrm{E} 20$. The TOUGH2 simulation proceeds until a convergence failure occurs at an unknown point in time $t_{c f}$, creating the steady-state regime. The primary variables at that point are written to file $S A V E$ to be used as initial conditions for the subsequent TOUGH2 simulation (only if keyword SAVE is present). The simulation time is then set to zero, and the transient regime of the simulation is initiated. This requires that boundary conditions are changed at time zero, e.g., by starting injection or withdrawal, or by changing Dirichlet-type boundary conditions (see command >> RESTART TIME).

(Note that if transient data are combined with steady-state data, the standard deviation of the steady-state data point may have to be decreased substantially to outweigh the large number of transient points.)

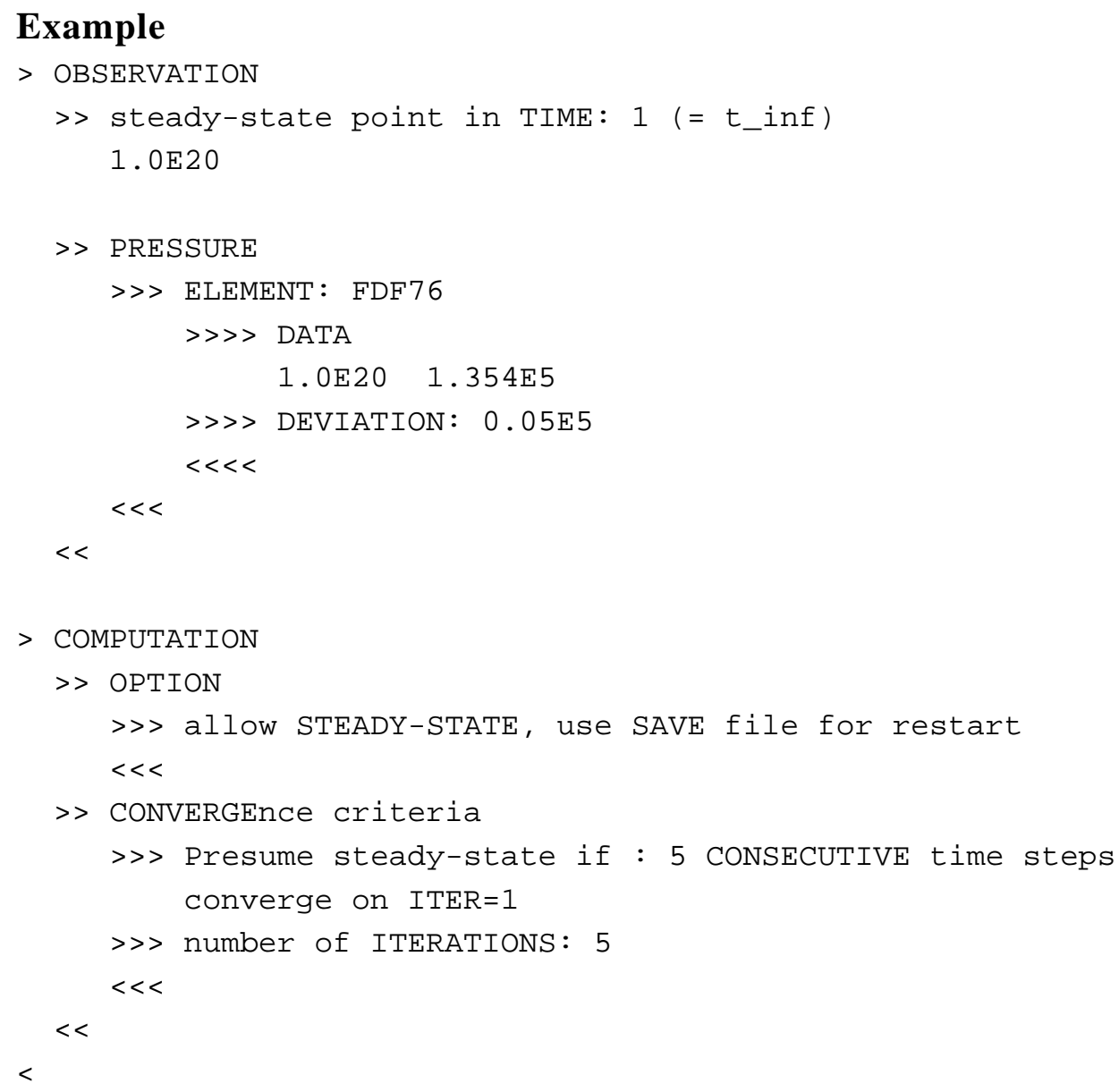




\section{Command}

>> STEP (UNSCALED) : max_step

\section{Parent Command}

$>$ CONVERGENCE

\section{Subcommand}

\section{Description}

This command defines the maximum allowable size of the scaled ( $f_{i}=p_{i}$, default) or unscaled ( $f_{i}=1$, keyword UNSCALED) update vector $\Delta \mathbf{p}$ per iTOUGH2 iteration. The step length of the scaled or unscaled parameter update vector is defined as follows:

$$
|\Delta \mathbf{p}|=\left[\sum_{i=1}^{n}\left(\frac{\Delta p_{i}}{f_{i}}\right)^{2}\right]^{1 / 2}
$$

This is a global step size limitation as opposed to the one specified for each individual parameter (see command $>>>>S T E P$ ). Limiting the global step size may make the inversion more stable. Parameter max_step should be chosen large enough, so that the size of the proposed step size $\left|\Delta \mathbf{p}^{\prime}\right|$ is reduced to $\left|\Delta \mathbf{p}_{\max }\right|$ only during the first few iterations. The figure below illustrates that limiting the step size maintains the direction of the step taken in the parameter space.

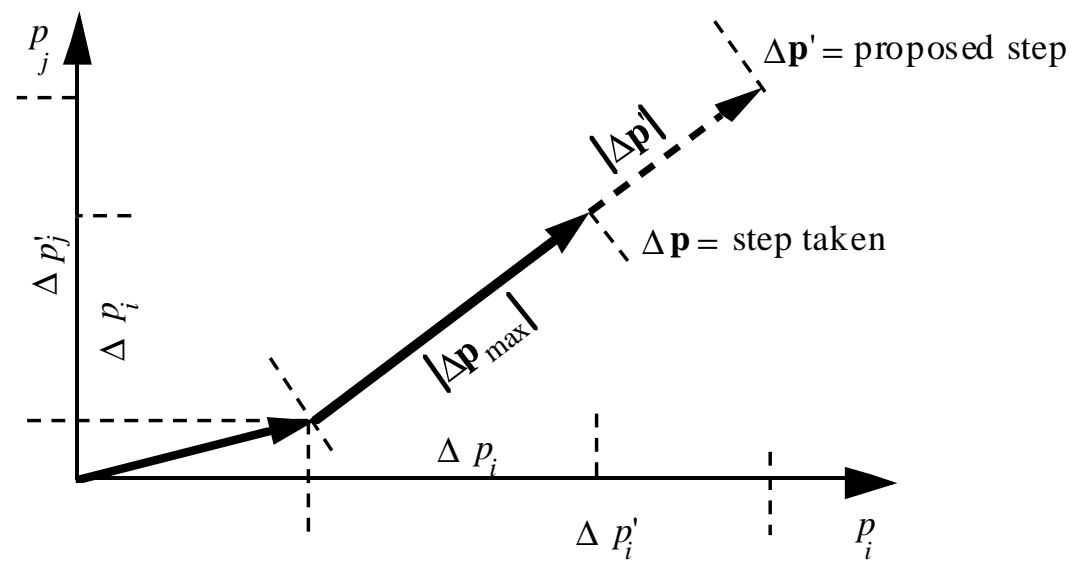

\section{Example}

$>$ COMPUTATION

$>>$ CONVERGENCE

$>>$ number of ITERATIONS : 10 (may need more iterations!)

$>>$ limit scaled STEP size to: 1.0 [dimensionless]

$<<<$

$<<$

See Also

$>>>$ STEP 


\section{Command}

>>> STEP: max_step

Parent Command

all third-level commands in block > PARAMETER

\section{Subcommand}

\section{Description}

This command defines the maximum allowable step size, limiting the change in the corresponding parameter per iTOUGH2 iteration. Large steps are usually proposed for a parameter if (i) the initial guess is far away from the best estimate, (ii) the parameter has a low sensitivity, or (iii) the parameter is highly correlated to more sensitive parameters. If the system state is a strongly non-linear function of the parameter, even a moderate step size may make the inversion unstable. Parameter max_step should be chosen large enough, so that the proposed step size $\Delta p_{i}^{\prime}$ is reduced to $\Delta p_{i, \max }$ only during the first few iterations. The figure below illustrates that limiting the step size also changes the direction of the step taken in the parameter space. A global step size limitation can also be specified (see command >> STEP), maintaining the direction of vector $\Delta \mathbf{p}^{\prime}$.

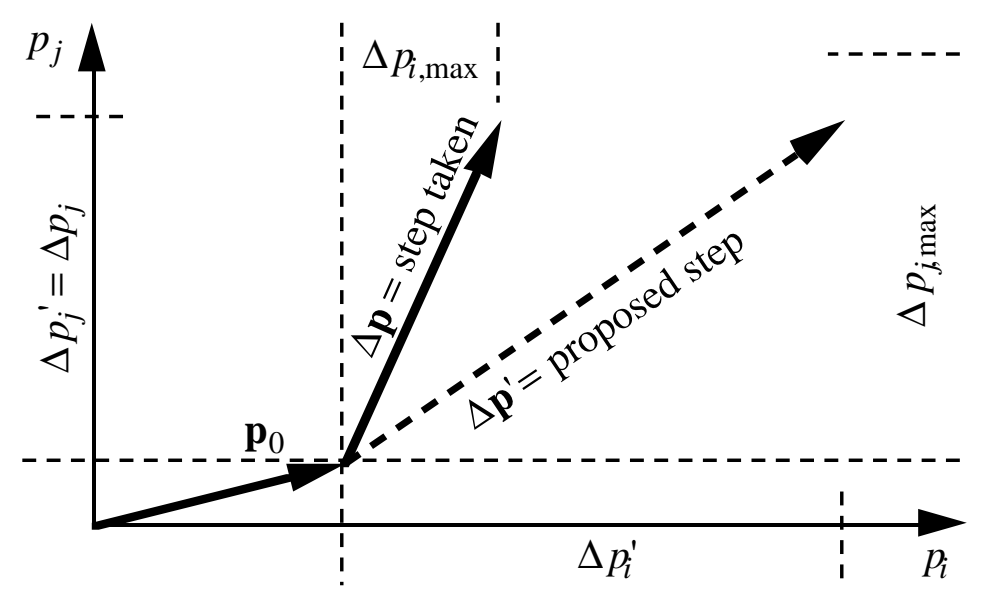

\section{Example}

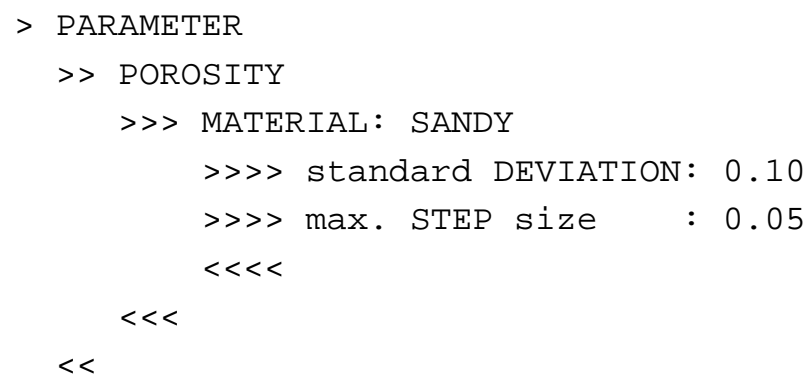

See Also

>> STEP, >>> DEVIATION, >>> RANGE 


\section{Command}

>>> STEP: max_step

\section{Parent Command}

$>>$ ANNEAL

\section{Subcommand}

$-$

\section{Description}

This command defines an iteration for Simulated Annealing minimization based on the number of successful steps or total number of steps as defined by max_step. After each iteration is completed, the temperature is reduced according to the annealing schedule (see command $>>>>$ SCHEDULE). An iteration is completed and the annealing temperature reduced if:

(1) the total number of successful and unsuccessful steps reaches max_step, or

(2) $(0.2 \cdot$ max_step $)$ successful steps have been performed

\section{Example}

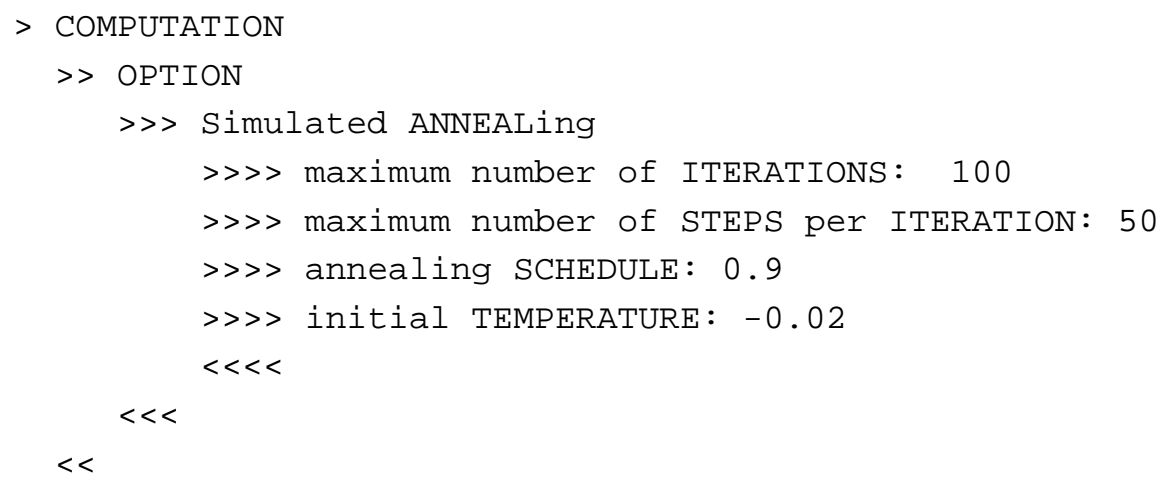

See Also

>>> SCHEDULE 
Command

>> STOP

Parent Command

$>$ COMPUTATION

Subcommand

(see command >> CONVERGE)

Description

(synonym for command $>$ CONVERGE)

Example

(see command $>$ CONVERGE)

See Also

>> CONVERGE 


\section{Command}

$>>>$ SUM

\section{Parent Command}

all third-level commands in block > OBSERVATION

\section{Subcommand}

$-$

\section{Description}

If multiple elements or connections are provided to indicate the location of a measurement point, this command takes the sum of all calculated values as the model output to be compared to the data. The user must ensure that the summation of the quantity is meaningful.

\section{Example}

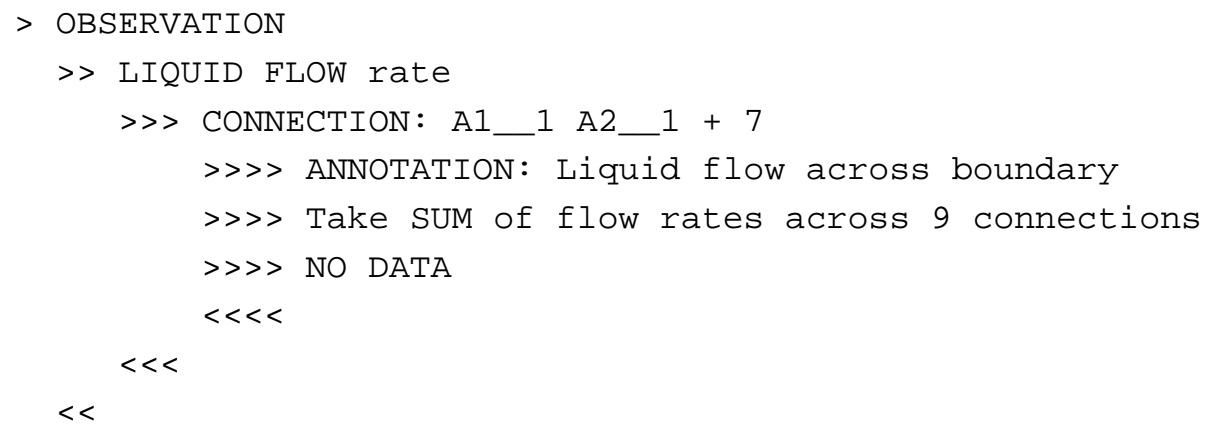

See Also

>>> AVERAGE 


\section{Command}

>> TAU: (-) niter

\section{Parent Command}

$>$ ERROR

\section{Subcommand}

\section{Description}

If multiple observation types (e.g., pressure, flow rate, prior information, etc.) are used to estimate model parameters, the relative weights assigned to each type is given by the ratio $\lambda_{i j}=\tau_{i} / \tau_{j}$, where $\tau_{i}$ are scalars such that $\mathbf{C}_{i}=\tau_{i} \mathbf{V}_{i}$. Here, $\mathbf{C}_{i}$ is the covariance matrix of all observations of type $i$ (a submatrix of covariance matrix $\mathbf{C}_{z z}$ ), and $\mathbf{V}_{i}$ is a positive symmetric matrix. By default, $\tau_{i}$ is fixed at 1 . This command allows $\lambda_{i j}$ to be updated in an iterative process, where $\tau_{i}$ is recalculated every multiple of niter iterations and is given by:

$$
\tau_{i}=\frac{\mathbf{r}_{i}^{T} \mathbf{C}_{i}^{-1} \mathbf{r}_{i}}{m_{i}}
$$

This procedure is similar to that sometimes referred to as iterated re-weighted least squares [Haining, 1990]. If a negative number is provided for niter, prior information is excluded from the process.

Recall that $\tau_{i}$ refers to all data of a certain observation type (i.e., not to individual data sets). The process assigns weights such that the relative contribution of each observation type to the objective function tends to $1 / \omega$, where $\omega$ is the number of observation types used in the inversion. It should also be realized that the Fisher model test becomes meaningless if $\lambda_{i j}$ is updated during the inversion because the test will be fulfilled by definition.

While this option provides flexibility in reassigning relative weights to data of different types, it is instead suggested to carefully select the standard deviations of the observations and prior parameter estimates (see command $>>>>$ DEVIATION $(\mathrm{p} / \mathrm{O})$ ) based on potential measurement and modeling errors.

\section{Example}

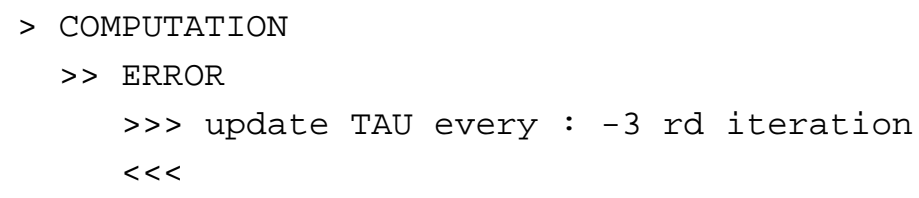

See Also

$>>>$ DEVIATION 


\section{Command}

>> TEMPERATURE

Parent Command

> OBSERVATION

\section{Subcommand}

>> ELEMENT

\section{Description}

This command selects temperature as an observation type. This observation type refers to an element.

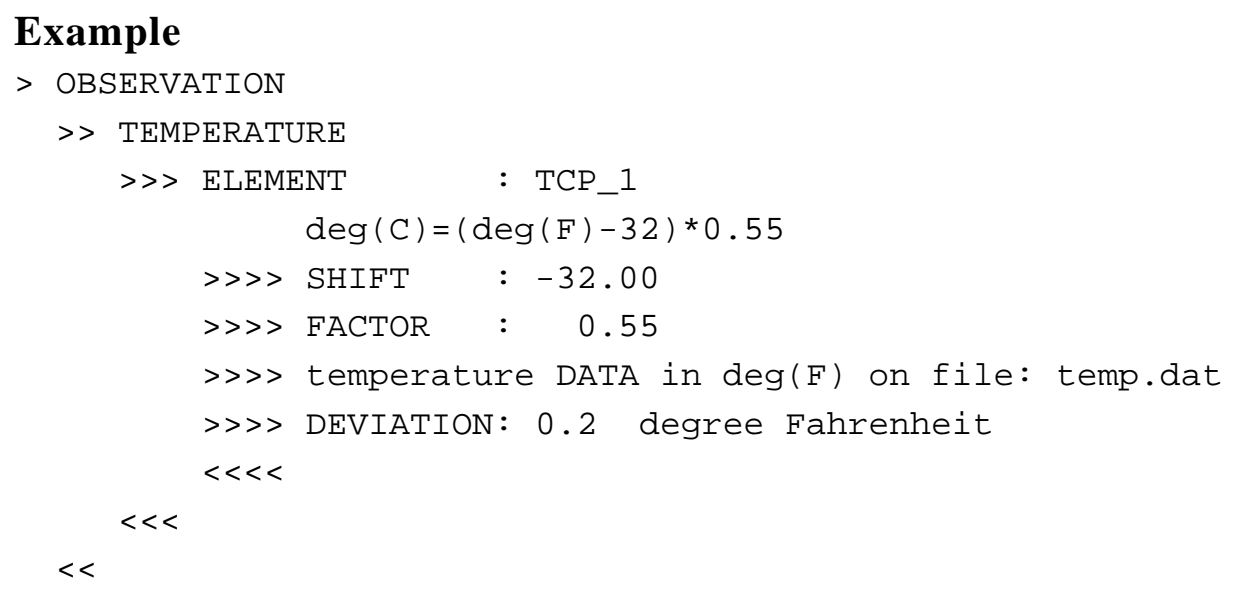

See Also 


\section{Command}

>>> TEMPERATURE: (-) temp0

\section{Parent Command}

$>>$ ANNEAL

\section{Subcommand}

$-$

\section{Description}

This command defines the initial temperature $\tau_{0}$ for Simulated Annealing minimization. The initial temperature is reduced after each iteration $k$ according to the annealing schedule (see command $>>>>$ SCHEDULE). The temperature $\tau_{k}$ defines the probability $\Pi$ with which an uphill step $\Delta S$ should be accepted:

$$
\Pi=\exp \left(-\Delta S / \tau_{k}\right)
$$

The probability decreases with decreasing temperature. The initial temperature temp 0 should be a reasonable fraction of the objective function $S$. If a positive value is given, then $\tau_{0}=$ temp 0 . If a negative value is provided, the initial temperature is internally calculated as a fraction of the initial objective function:

$$
\tau_{0}=|\operatorname{temp} 0| \cdot S_{0}
$$

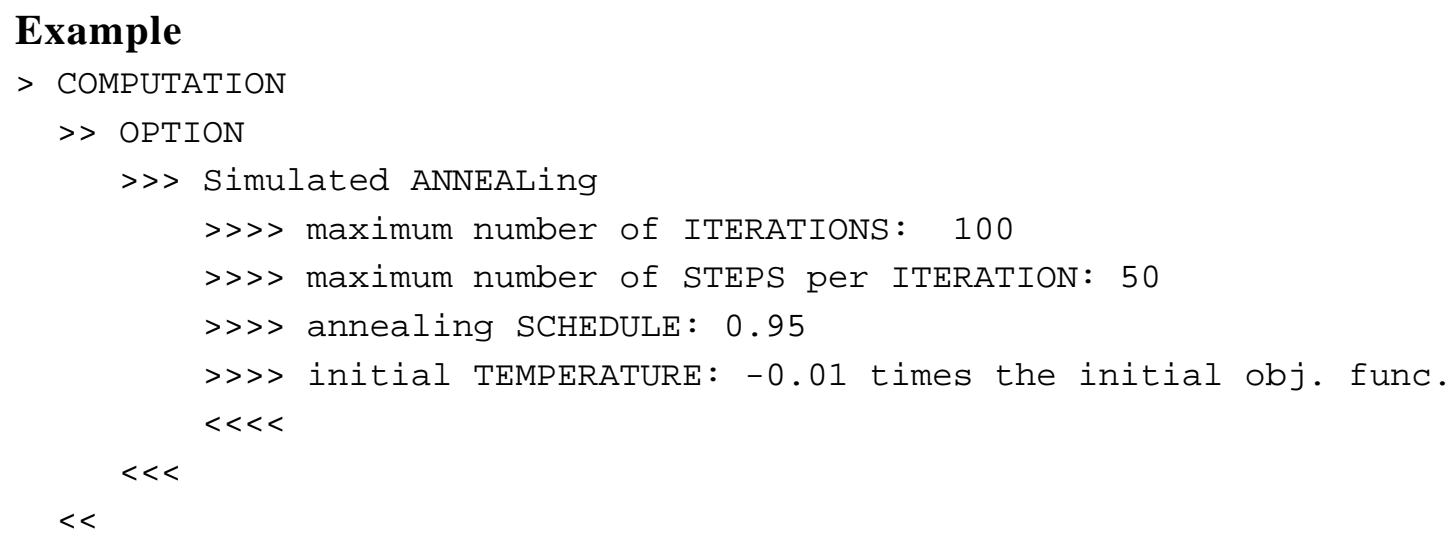

See Also

>>> STEP (a), >>> SCHEDULE 


\section{Command}

$>$ TIME

\section{Parent Command}

$>$ PARAMETER

\section{Subcommand}

>> SOURCE

\section{Description}

This command selects as a parameter the time of a time-dependent generation rate (TOUGH2 variable $F 1(L)$ for $L T A B>1$ ). This parameter refers to a sink/source code name. Index $L$ must be provided through command $>>>>$ INDEX. Time stepping during simulation should be small compared to the expected variation of the parameter. Furthermore, it is suggested to prescribe the parameter perturbation for calculation of the Jacobian, i.e. use command $>>>>$ PERTURB and specify a reasonably large time perturbation provided as a negative number.

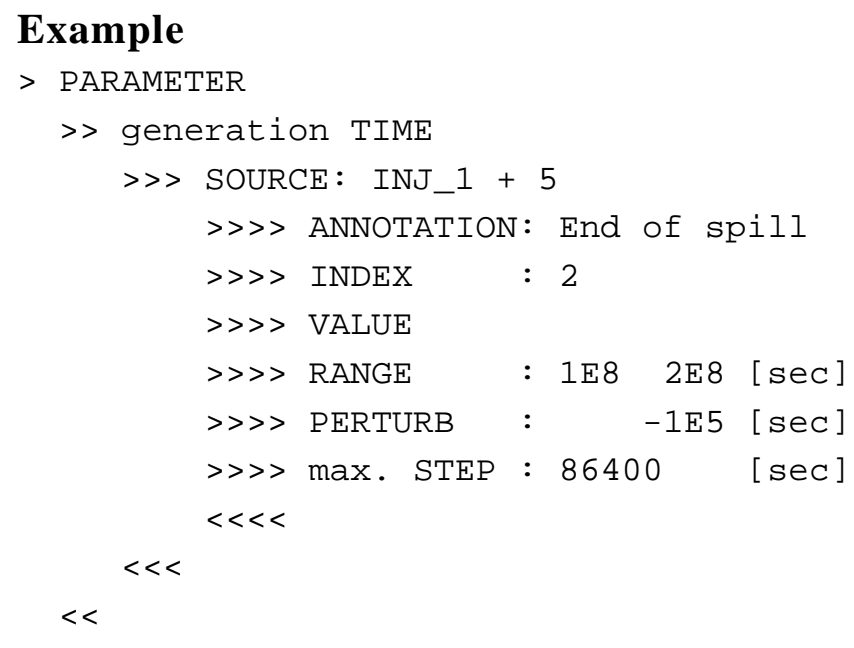

See Also

$>$ ENTHALPY, >> RATE 


\section{Command}

>> TIME: ntime (EQUAL/LOGARITHMIC) (time_unit)

Parent Command

$>$ OBSERVATION

\section{Subcommand}

\section{Description}

This command selects points in time during a TOUGH2 run at which the calculated system response is used for subsequent iTOUGH2 analysis. In the case of inverse modeling, these are the calibration times at which calculated and observed system responses are compared. Time stepping in the TOUGH2 simulation is forced to exactly match all specified calibration times, i.e., defining calibration times has the same effect on the simulation as specifying printout times in the TOUGH2 block TIMES (printout can be suppressed, however, by specifying a negative integer for TOUGH2 parameter KDATA). Thus, time stepping considerations must be taken into account when selecting the spacing of calibration points (see also command >>> CONSECUTIVE).

Only one set of calibration times can be specified which is then applied to all data sets (see, however, command >>>> WINDOW).

Calibration times do not have to match the times at which measurements are available, since the value of the observed data at the calibration point will be linearly interpolated between adjacent data points as illustrated in the figure below.
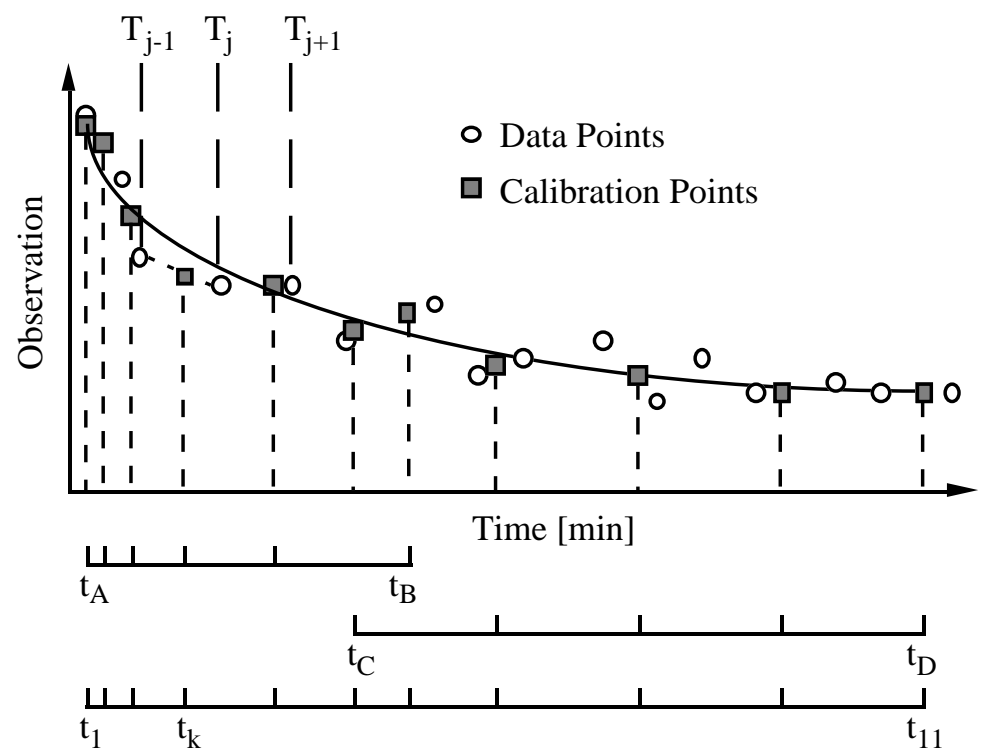
Calibration times can be specified in three ways: (i) explicitly listed, (ii) automatically generated with equal spacing, and (iii) automatically generated with logarithmic spacing. The corresponding input formats are given by example below. Admissible keywords for selecting the time units time_unit are SECOND, MINUTE, HOUR, DAY, WEEK and YEAR.

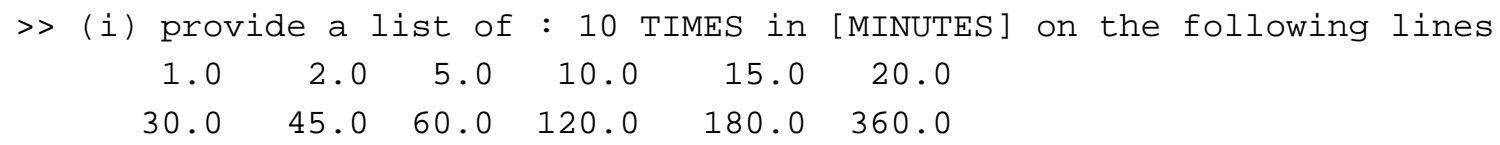

In the example above, the first ntime $=10$ numbers will be read in free format and interpreted as calibration times in minutes.

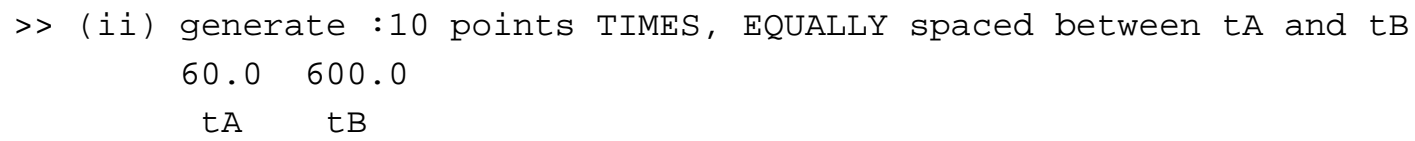

10 equally spaced points in time will be generated between 60.0 and 600.0 seconds. Time limits $t A$ and $t B$ are read in free format.

> (iii) generate:10, LOGARITHMICALLY spaced points TIMES in [HOURS]
0.0112 .0

10 logarithmically spaced points in time will be generated between 36 seconds and 12 hours.

The three formats can be used repeatedly, concurrently, and with overlapping time periods (see example below). All specified times will be sorted internally.

\section{Example}

The following block generates a total of 11 calibration times; they are schematically shown in the figure above.

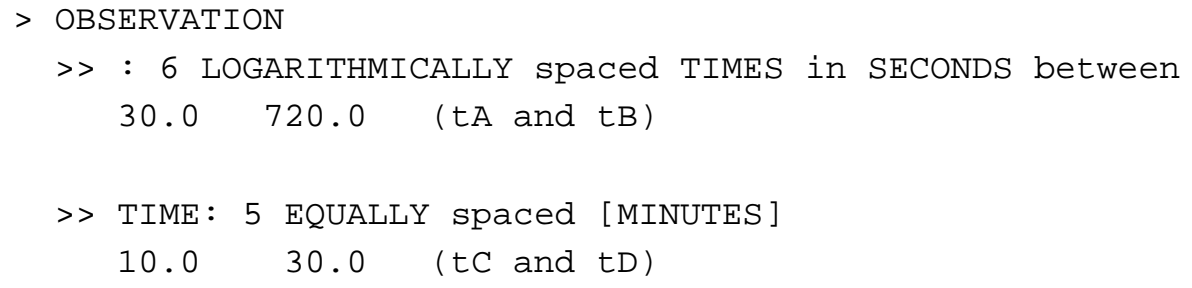

See Also

$>$ RESTART TIME, $>>$ CONSECUTIVE, $>>>>$ WINDOW 


\section{Command}

>> time_unit

or as a keyword to any command requesting time units

\section{Parent Command}

$>$ OUTPUT

\section{Subcommand}

\section{Description}

This command or keyword specifies the time units of all times in the iTOUGH2 output and plot files. Admissible time units are SECOND (default), MINUTE, HOUR, DAY, WEEK, MONTH, and YEAR. The time output unit may be different from the time units specified in the input or data files.

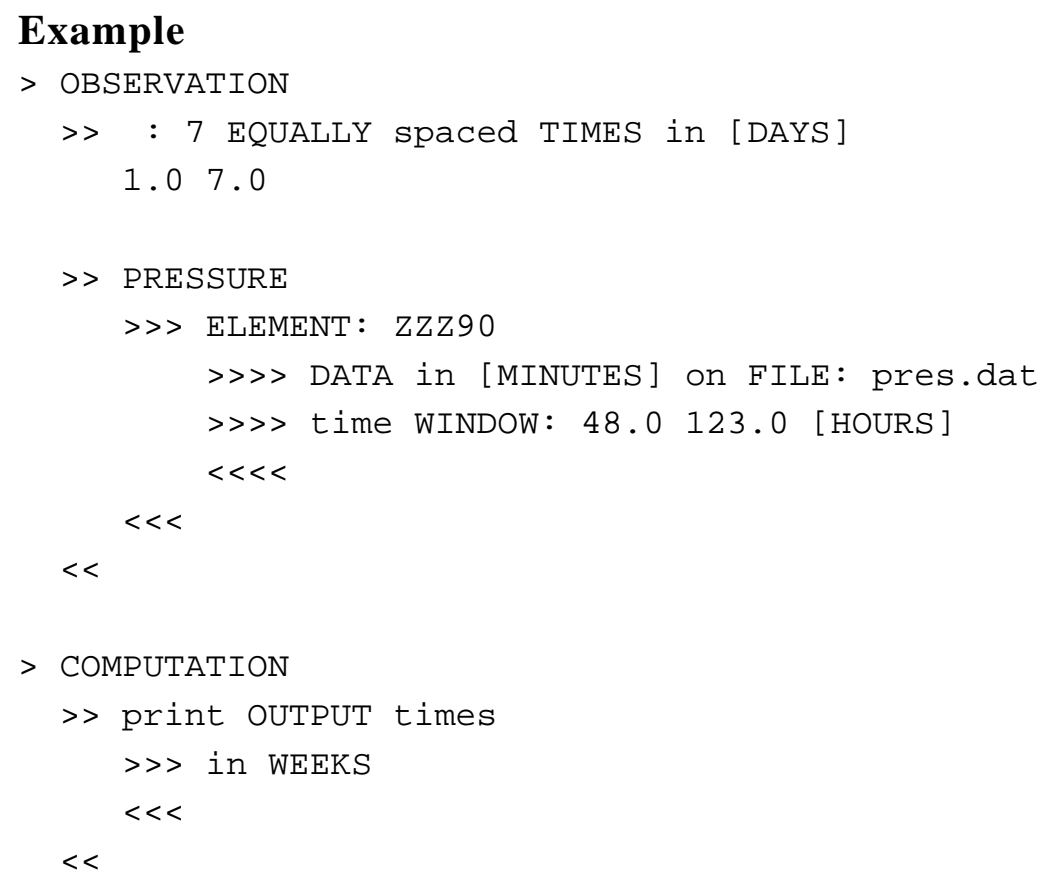

See Also 
Command

$>$ TOLERANCE

Parent Command

$>$ COMPUTATION

Subcommand

(see command $>$ CONVERGE)

Description

(synonym for command $>$ CONVERGE)

Example

(see command $>$ CONVERGE)

See Also

>> CONVERGE 


\section{Command}

>> TOTAL MASS (phase_name/PHASE: iphase/

comp_name/COMPONENT: icomp) (CHANGE)

\section{Parent Command}

$>$ OBSERVATION

\section{Subcommand}

$>>$ MATERIAL

$>>$ MODEL

\section{Description}

This command selects as an observation type the total mass of phase iphase or component icomp. This observation type refers either to the entire model domain (command >> MODEL, see also Section A8), or to the subdomain specified by a list of rock types (command >>> MATERIAL).

Component number icomp or component name comp_name, and phase number iphase or phase name phase_name depend on the EOS module being used. They are listed in the header of the iTOUGH2 output file, and can be specified either on the command line or using the two subcommands >>>> COMPONENT and >>>> PHASE, respectively. If keyword CHANGE is present, the change of the total mass since data initialization is computed.

\section{Example}

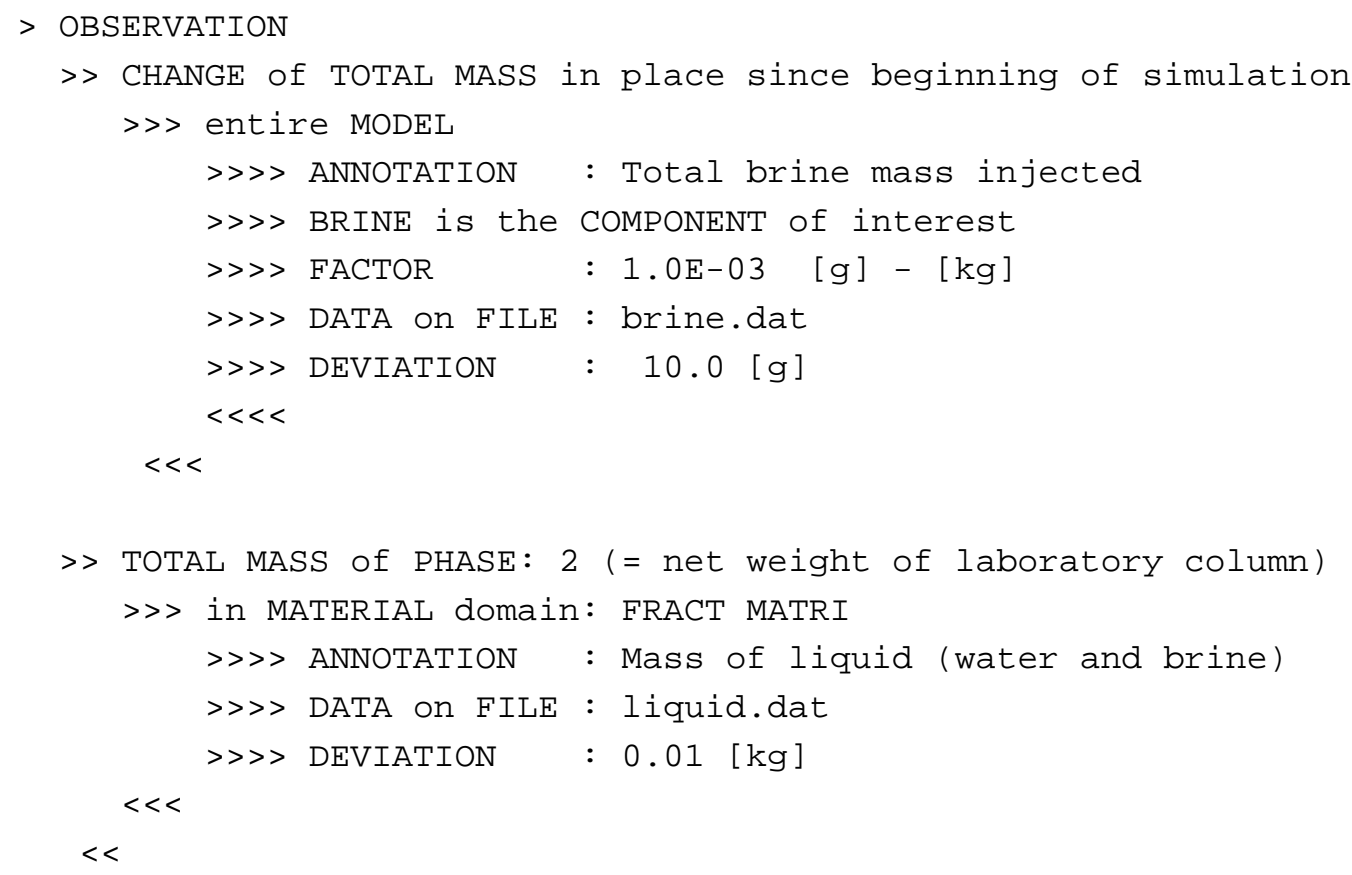

\section{See Also}




\section{Command}

$>>>$ UNIFORM

\section{Parent Command}

all third-level commands in block > PARAMETER

\section{Subcommand}

$-$

\section{Description}

This command generates uniformly distributed input parameters for Monte Carlo simulations. Parameter values are sampled between the lower and upper bound specified by command $>>>>$ RANGE.

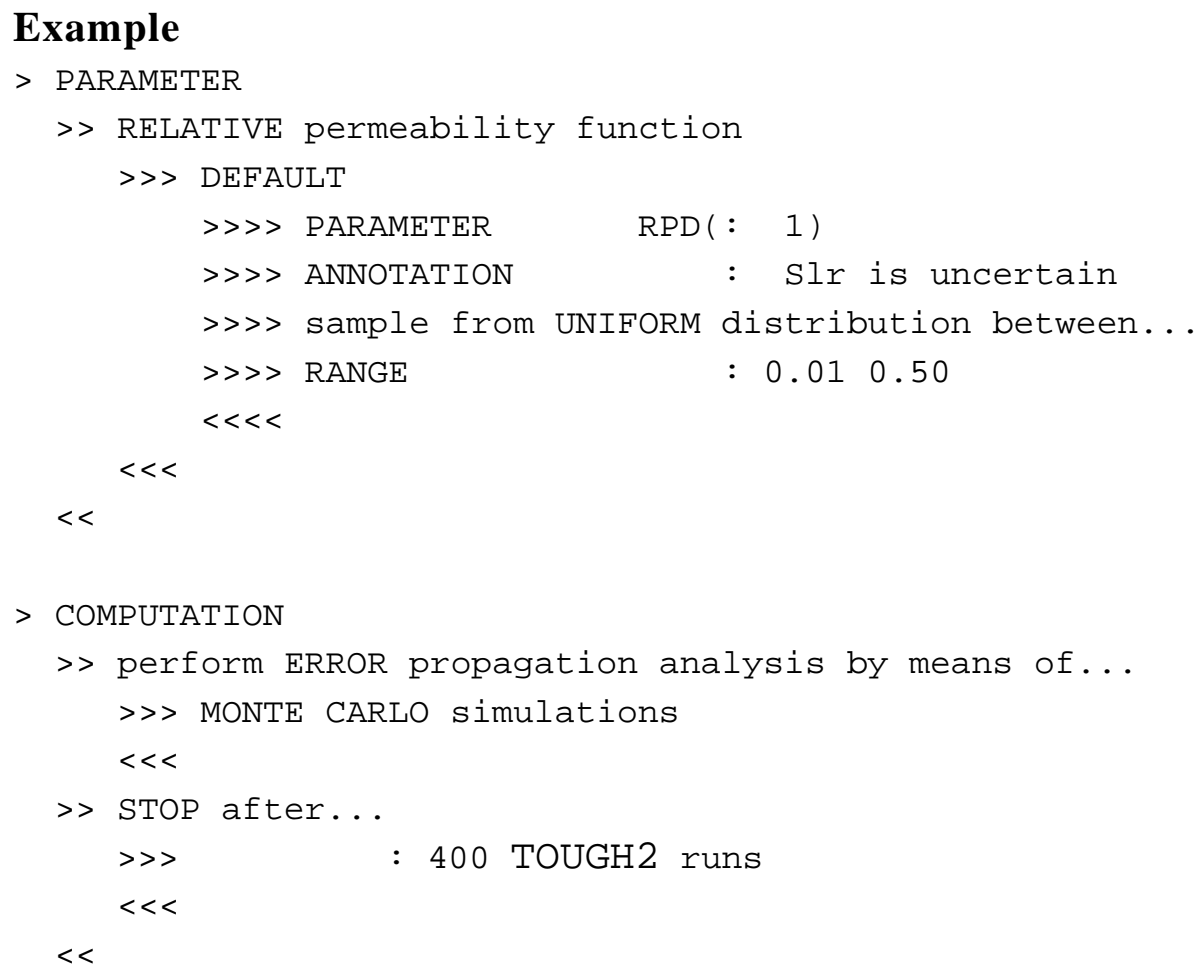

See Also

>> MONTE CARLO, $>>>$ GAUSS 


\section{Command}

>> UPDATE

\section{Parent Command}

$>$ OUTPUT

Subcommand

\section{Description}

This command writes version update information to the iTOUGH2 output file.

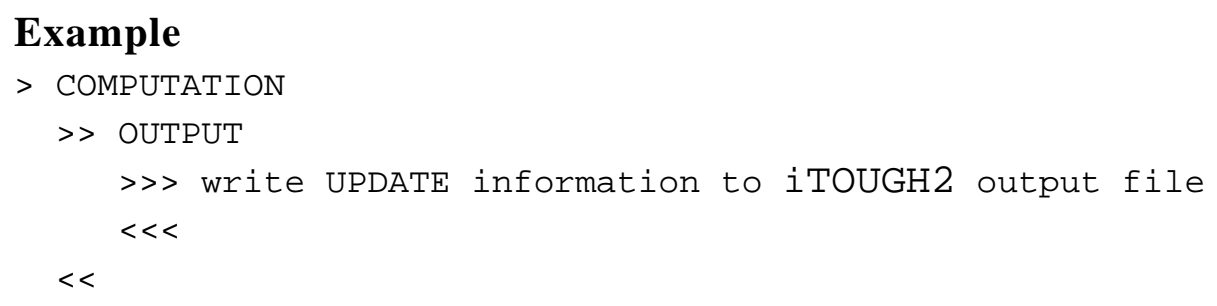

See Also

>> INDEX, >> VERSION, HELP, LIST 


\section{Command}

>>> UPHILL: max_uphill

\section{Parent Command}

>> CONVERGE

\section{Subcommand}

$-$

\section{Description}

By default, an inversion is stopped if more than 10 consecutive unsuccessful parameter steps have been proposed. The default can be overwritten by max_uphill. Each unsuccessful step results in an increase of the Levenberg parameter $\lambda$ (see command $>>>$ LEVENBERG), leading to a smaller step size deflected towards the gradient.

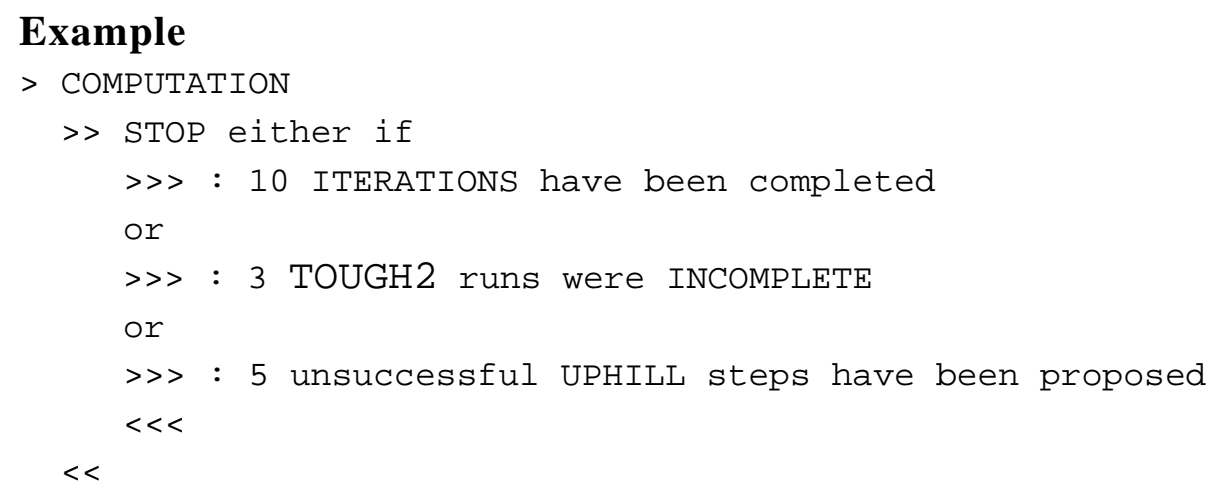

See Also

>> ITERATION, >>> LEVENBERG 


\title{
Command
}

>> USER (: anno)

\section{Parent Command}

$>$ PARAMETER

\author{
Subcommand \\ $>>$ ELEMENT \\ $>>>$ MATERIAL \\ $>>$ MODEL \\ $>>\mathrm{SET}$ \\ $>>$ SOURCE
}

\section{Description}

This command selects a user-specified parameter. This option enables a user to estimate any conceivable parameter which can be any TOUGH2 variable or function thereof. The user must program the function in subroutine USERPAR, file it2user.f. Identification of and details about the parameter can be given in the iTOUGH2 input file and will be transferred to subroutine USERPAR. A parameter annotation anno should be provided following a colon on the command line. This annotation (or a substring thereof) will be available in subroutine USERPAR (variable $A N N O$ ) and can be used to identify the parameter type. The significant part of the string should therefore not be changed by command >>> ANNOTATION. Furthermore, multiple material names as well as grid block or sink/source code names defined by the corresponding third-level command will be transferred to the subroutine in array NAMEA. Integers read after command >>> INDEX are provided through array IDA. A flag (variable IVLF) indicates whether the estimate is a value, logarithm, or multiplication factor of the corresponding parameter.

The user must ensure that all TOUGH2 variables used by the function are transferred to subroutine USERPAR via COMMON blocks. If a variable is not predefined in one of the standard COMMON blocks, a new COMMON block must be created and added to the include file usercom.inc.

Subroutine USERPAR has two major blocks. In the first block ( IUIG=1), the value specified in the TOUGH2 input file is transferred to iTOUGH2 as an initial guess. Programming this first part is optional because initial guesses can also be provided through the iTOUGH2 input file by means of command >> GUESS, >>>> PRIOR, or >>> GUESS. Programming the second part $(I U I G=2)$ is mandatory. In this part, the parameter is updated, i.e., the generic parameter value calculated by iTOUGH2, which is stored in variable $X X$, must be assigned to the appropriate TOUGH2 variable.

The following is the header of subroutine USERPAR in file it 2 user.f, describing the transfer variables. File it2user. $f$ must be recompiled before the user-specified parameter becomes active. 


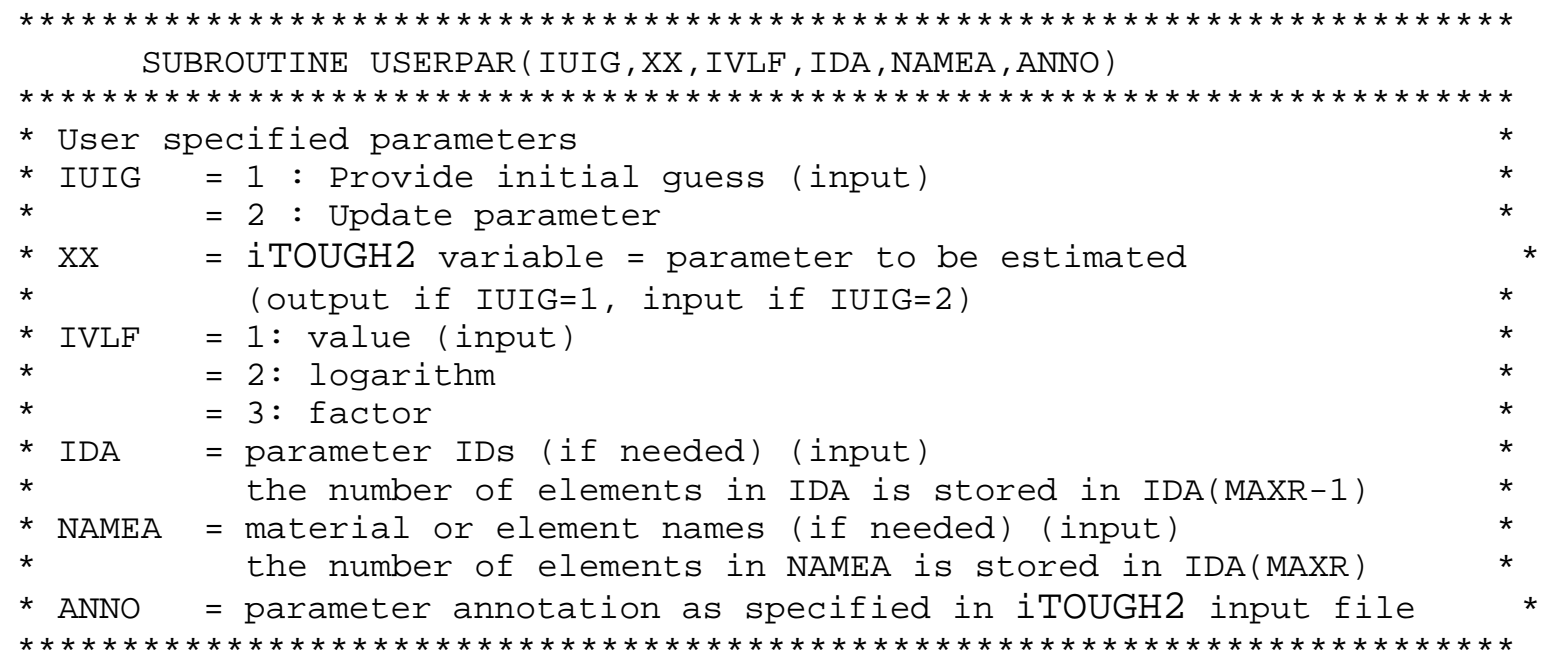

\section{Example}

In this simple example, the tortuosity factor (TOUGH2 variable TORT (NMAT)) is treated as a user-specified parameter. Variable TORT is provided through COMMON block SOLI1 1 in include file rock.inc. The parameter block in the iTOUGH2 input file and subroutine USERPAR are given below.

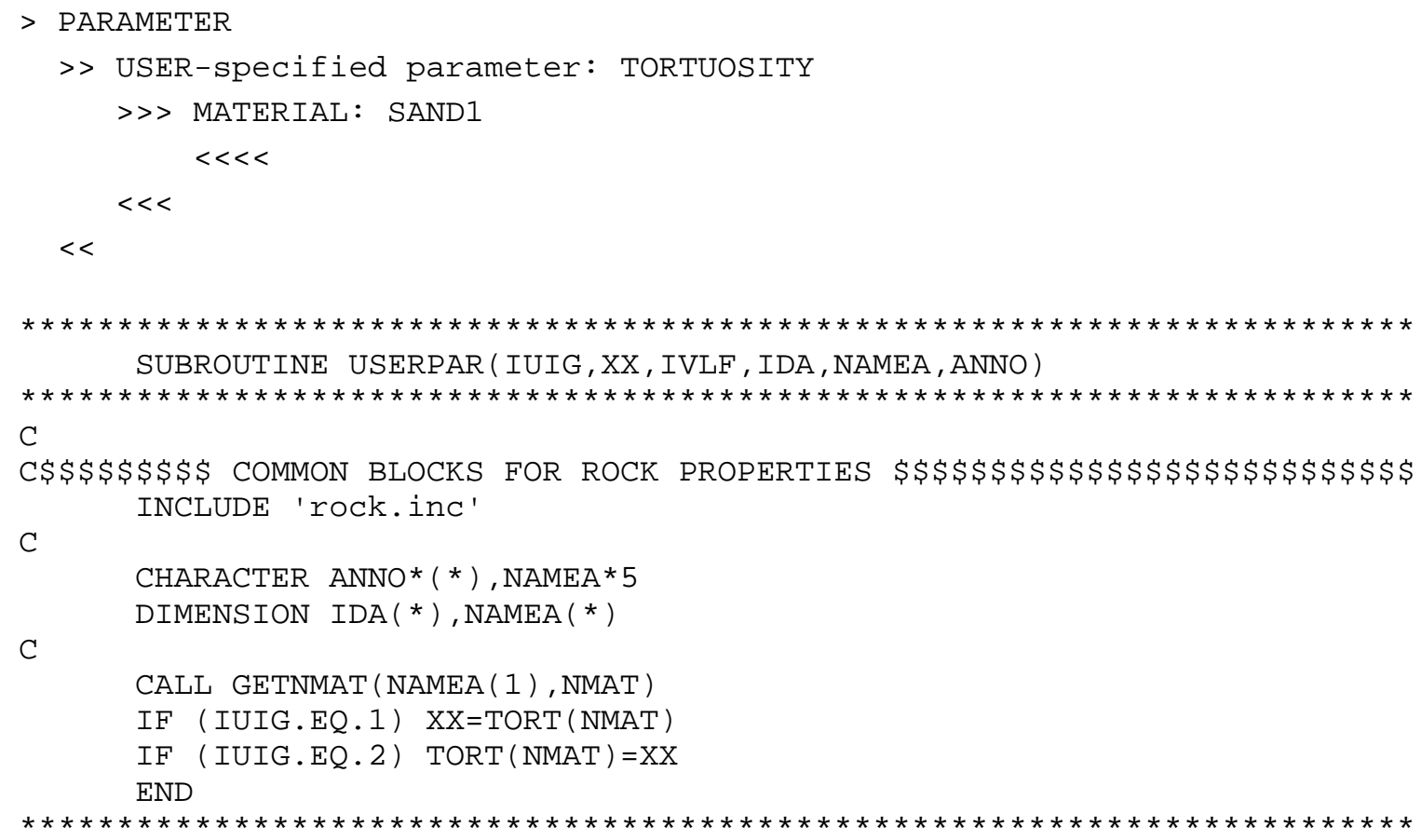

The following is a more general version of subroutine USERPAR for the determination of tortuosity. It allows specifying multiple rock types on command line $>>>>$ MATERIAL, and offers the possibility to estimate tortuosity either as a value, a logarithm, or a multiplication factor. Note that identifying the parameter by its annotation is only necessary if other user-specified parameters are also addressed in the same subroutine. 


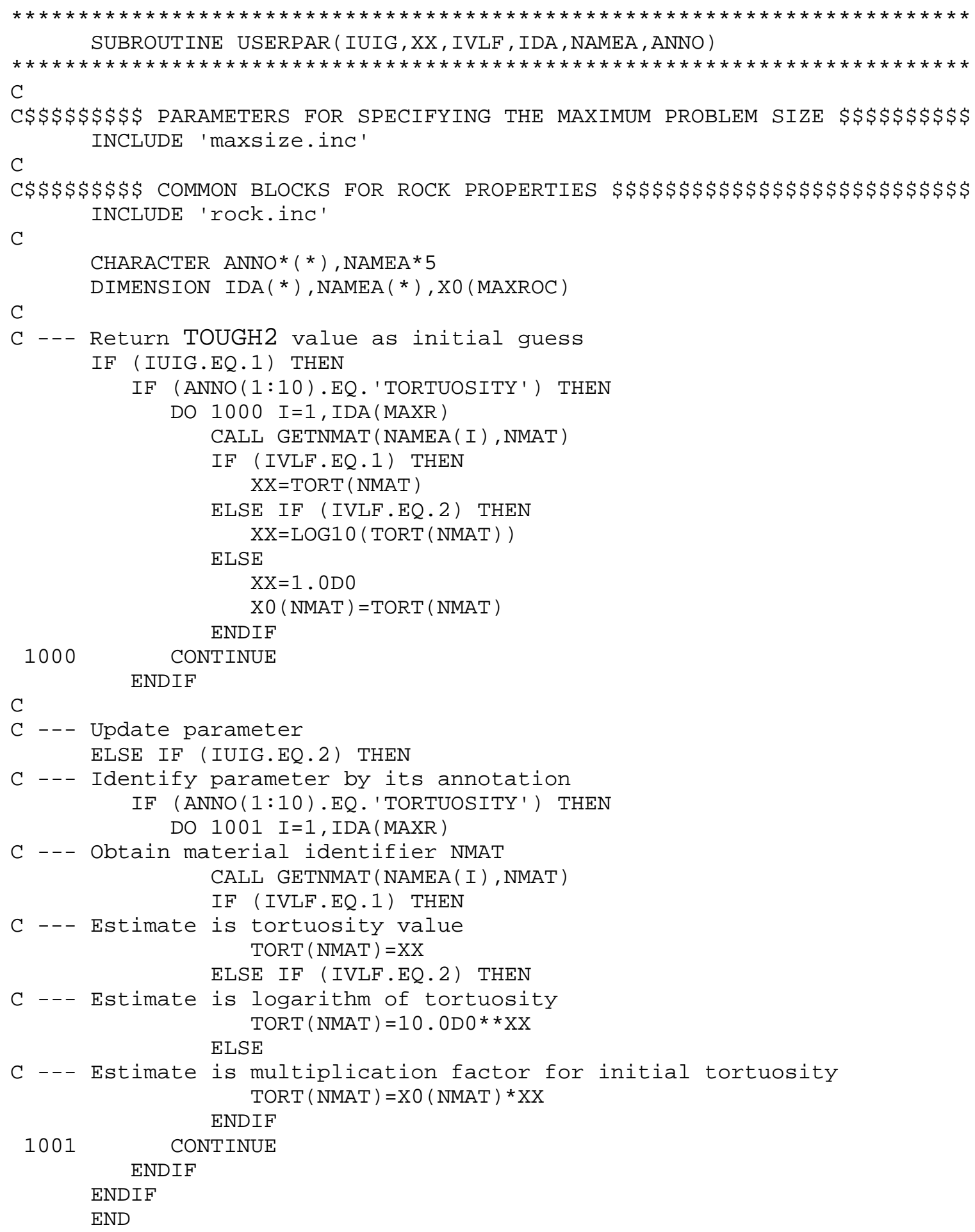

Additional examples can be found in file it2user.f .

\section{See Also}




\section{Command}

>> USER (: anno)

Parent Command

$>$ OBSERVATION

\section{Subcommand}

$>>$ CONNECTION

$>>$ ELEMENT

$>>$ MODEL

$>>$ SOURCE

\section{Description}

This command selects a user-specified observation type. This option enables a user to introduce a new observation type, i.e., any type of data can be used for parameter estimation by defining the corresponding model output which is a user-specified function of TOUGH2 variables. The user must program the function in subroutine USEROBS, file it2user.f. Identification of and details about the observation can be given in the iTOUGH2 input file and will be transferred to subroutine USEROBS. The annotation anno (or a substring thereof) will be available in subroutine USEROBS (variable $A N N O$ ); it can be used to identify the observation type and data set. The significant part of the string should therefore not be changed by subcommand >>> ANNOTATION. Multiple grid block names or sink/source code names defined by the appropriate third-level command will be transferred to the subroutine in array GRIDA. The corresponding element numbers are stored in array NECA, with INEC pointing to the currently processed array index. Integer variables read after command >>> INDEX are provided through array IOBSA. On output, subroutine USEROBS returns the user-specified model result TRESULT.

The user must ensure that all TOUGH2 variables used by the function are transferred to subroutine USERPAR via COMMON blocks. If a variable is not predefined in one of the standard COMMON blocks, a new COMMON block must be created and added to the include file usercom.inc.

The following is the header of subroutine USEROBS, describing the transfer variables. File it2user.f must be recompiled before the user-specified observation becomes active.

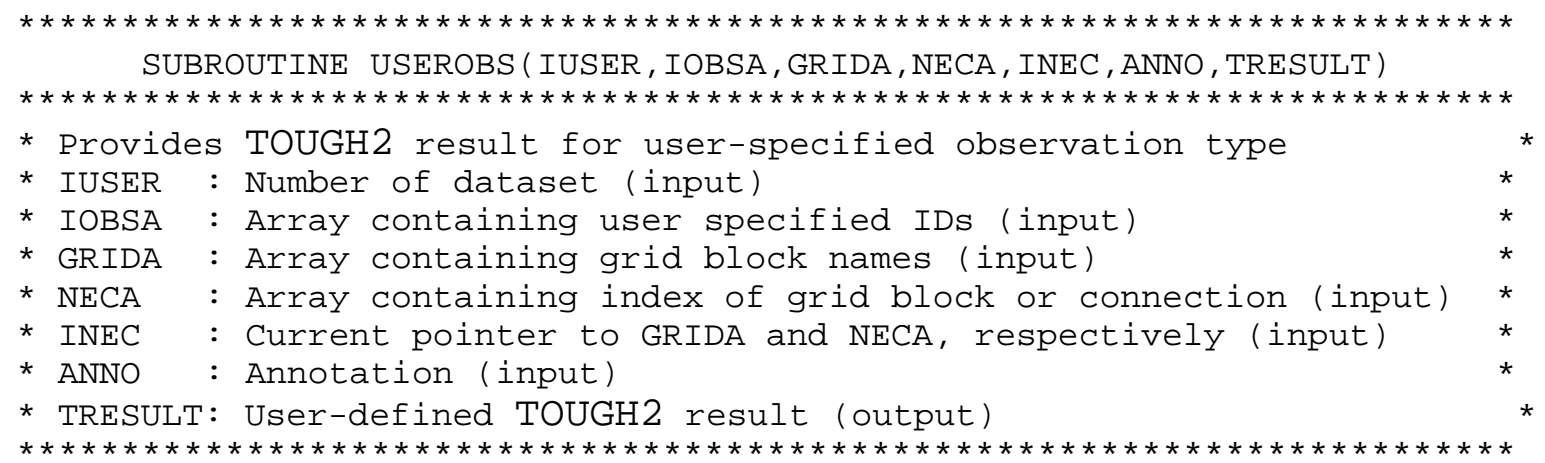




\section{Example}

In this example the pressure difference measured between two points in a laboratory column is defined as a user-specified observation. Two differential pressures are measured, referring to the liquid and NAPL phase, respectively. The iTOUGH2 input block and subroutine USEROBS are given below. Variable IOBSA (MAXR-2) holds the phase number.

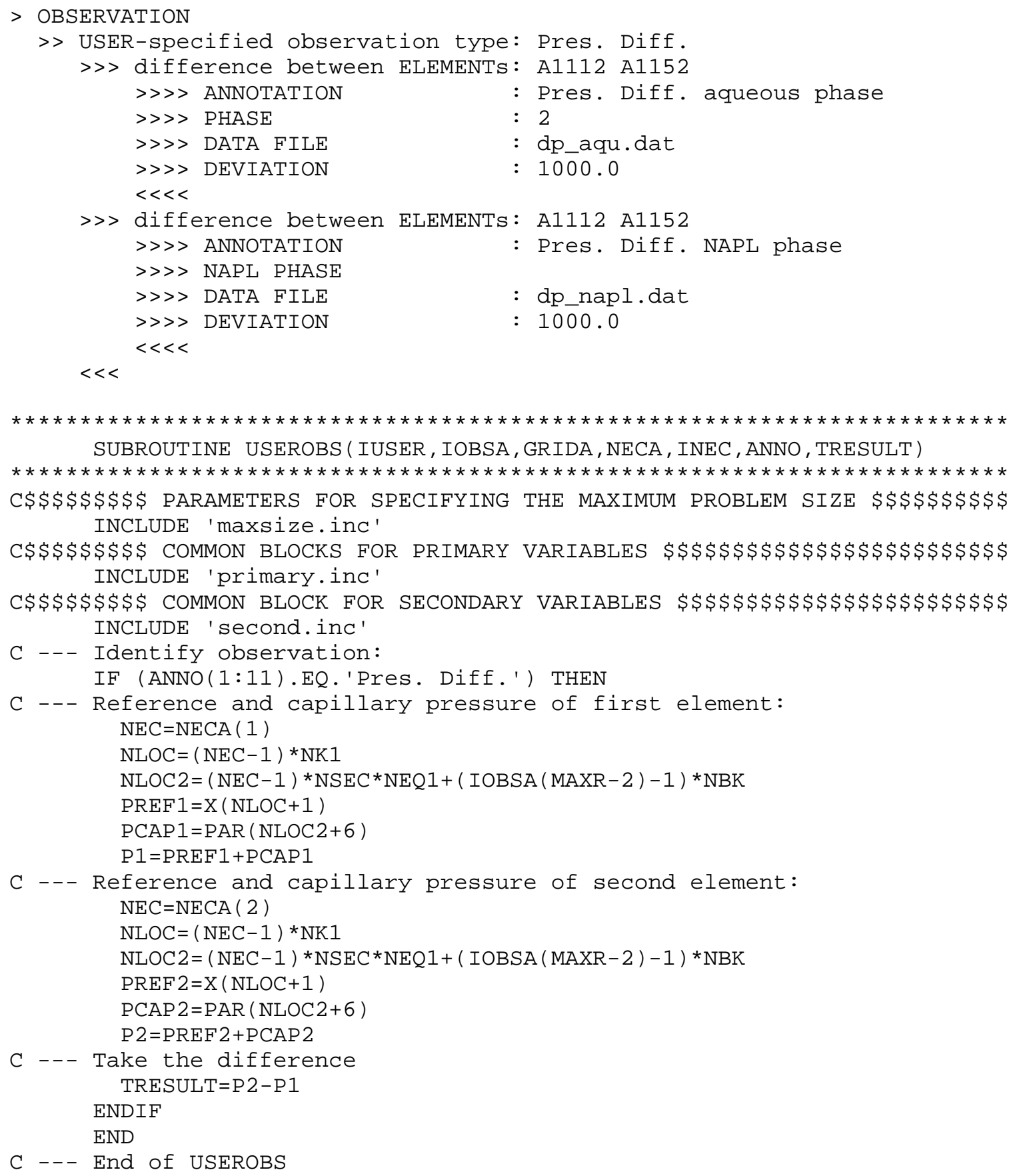

See Also 


\section{Command}

$>>>$ USER

Parent Command

all third-level commands in block > OBSERVATION

\section{Subcommand}

\section{Description}

Observed data can be represented as a user-specified function of time. The function must be coded into subroutine USERDATA, file it2user.f. The following is the header of subroutine USERDATA, describing the transfer variables. File it 2 user. $f$ must be recompiled before the user-specified data definition becomes active.

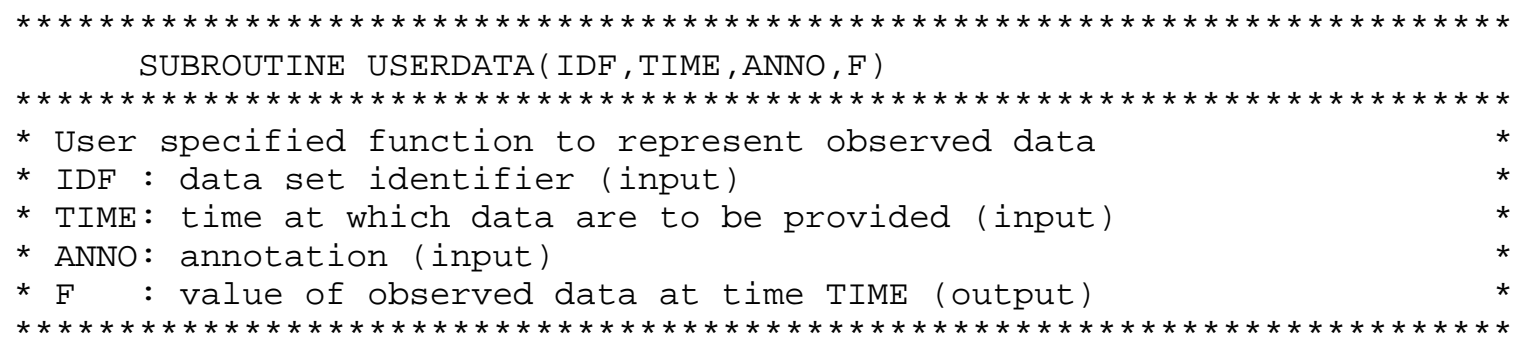

\section{Example}

In this example an analytical solution is provided as data to be matched (see sample problem 8). Liquid saturation in response to a liquid pulse is calculated as a function of time and location in subroutine USERDATA (see following page). Note that the Z-coordinate is passed to USERDATA through the annotation.

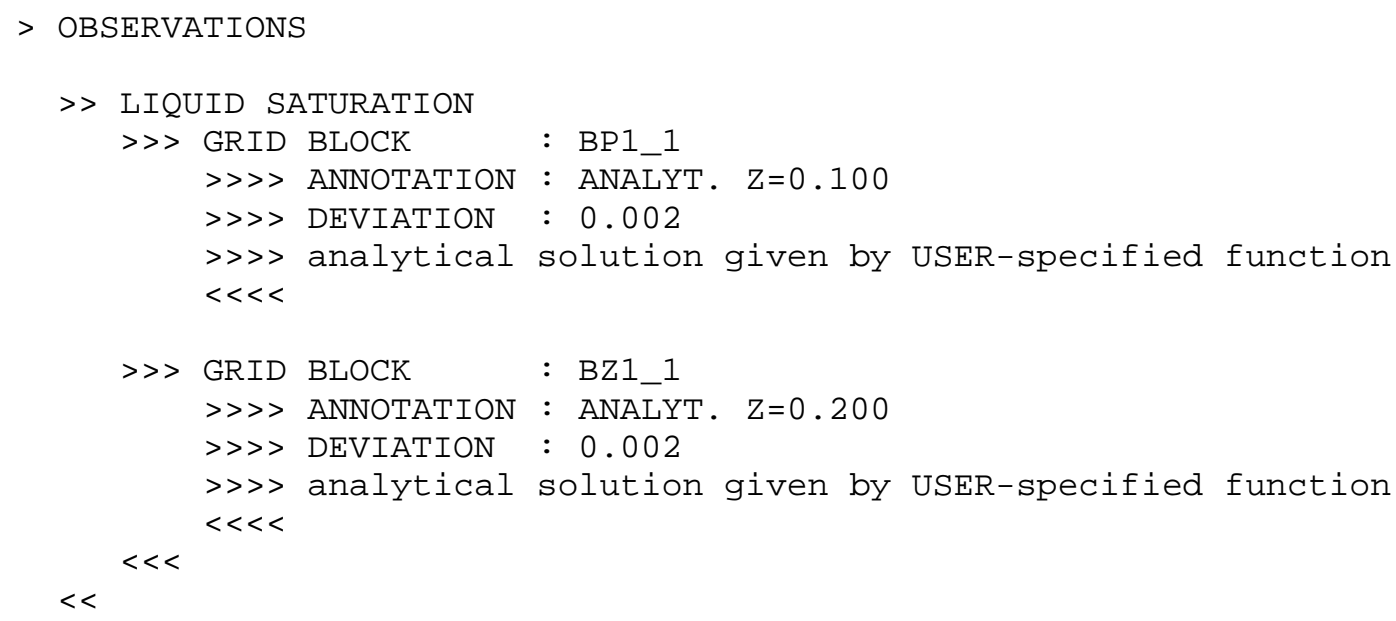




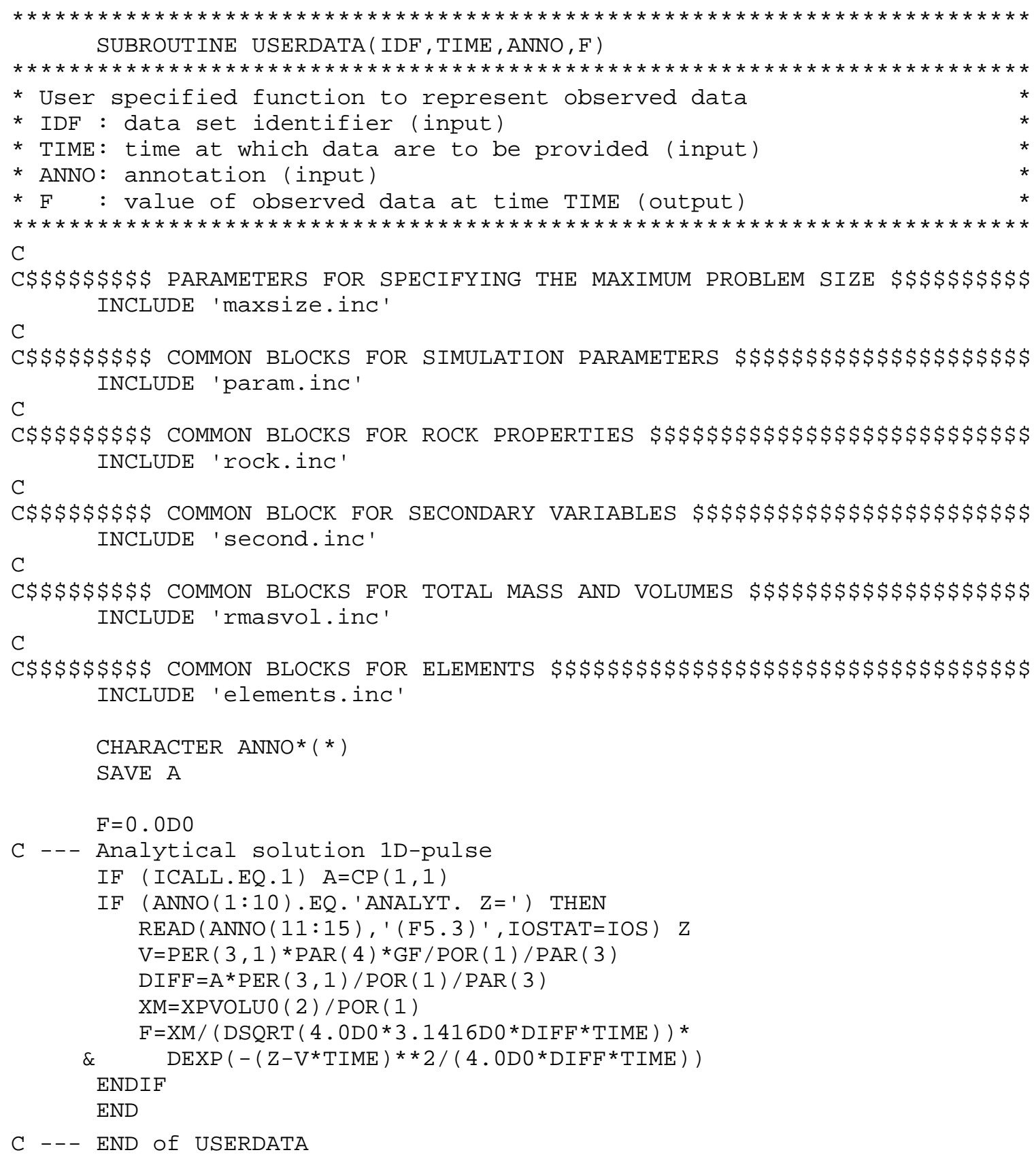

\section{See Also}

>>> DATA, >>>> POLYNOM 


\section{Command}

$>>>$ VALUE

\section{Parent Command}

all third-level commands in block > PARAMETER

\section{Subcommand}

$-$

\section{Description}

This command selects the estimation of the parameter value as opposed to its logarithm (see command $>>>>$ LOGARITHM $(\mathrm{p})$ ) or a multiplication factor (commands $>>>$ FACTOR $(\mathrm{p})$ or $>>>>$ LOG $(\mathrm{F}))$.

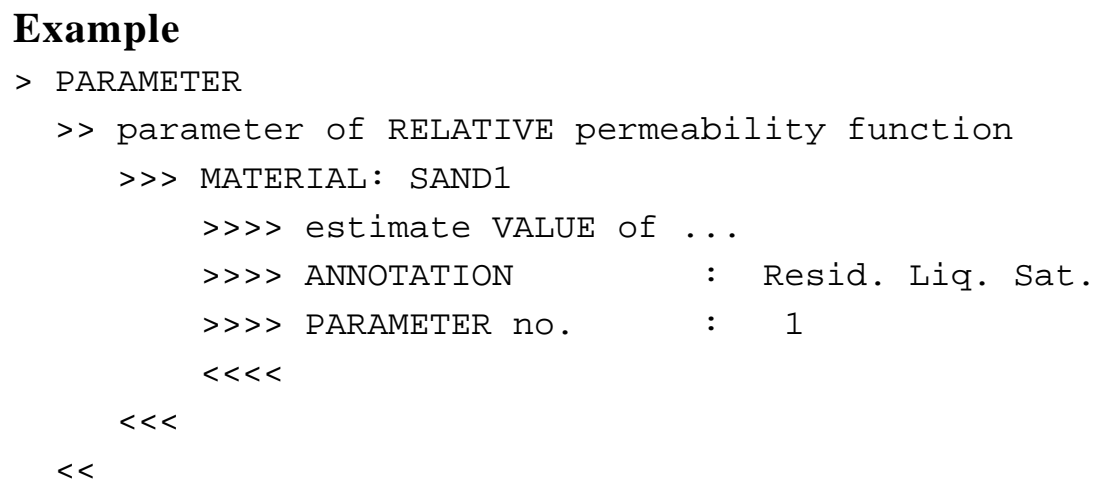

See Also

$>>>$ FACTOR $(\mathrm{p}),>>>>$ LOGARITHM $(\mathrm{p}),>>>>$ LOG $(\mathrm{F})$ 


\section{Command}

>>> VARIANCE: sigma^2

\section{Parent Command}

all third-level commands in block > PARAMETER and > OBSERVATION

\section{Subcommand}

$-$

\section{Description}

(see $>>>>$ DEVIATION $(\mathrm{p} / \mathrm{o})$ )

\section{Example}

$($ see $>>>$ DEVIATION $(\mathrm{p} / \mathrm{o}))$

\section{See Also}

$>>>$ DEVIATION ( $\mathrm{p} / \mathrm{o})$ 


\section{Command}

>>> VARIATION: sigma

\section{Parent Command}

all third-level commands in block > PARAMETER

\section{Subcommand}

\section{Description}

This command specifies the expected variation $\sigma_{p_{j}}$ of a parameter. $\sigma_{p_{j}}$ is used to scale the columns of the Jacobian matrix, yielding dimensionless and comparable sensitivity coefficients. While the solution of the inverse problem is not affected by the choice of the scaling factor, all the qualitative sensitivity measures are directly proportional to $\sigma_{p_{j}}$ :

$$
\text { scaled sensitivity coefficient: } S_{i j}=\frac{\partial z_{i}}{\partial p_{j}} \cdot \frac{\sigma_{p_{j}}}{\sigma_{z_{i}}}=J_{i j} \cdot \frac{\sigma_{p_{j}}}{\sigma_{z_{i}}}
$$

If no standard deviation (see $>>>>\operatorname{DEVIATION}(\mathrm{p})$ ) or parameter variation is specified, the scaling factor is taken to be $10 \%$ of the respective parameter value.

For sensitivity analyses, $\sigma_{p_{j}}$ can be taken as the perturbation one would apply to study the effect of the parameter on the modeling result.

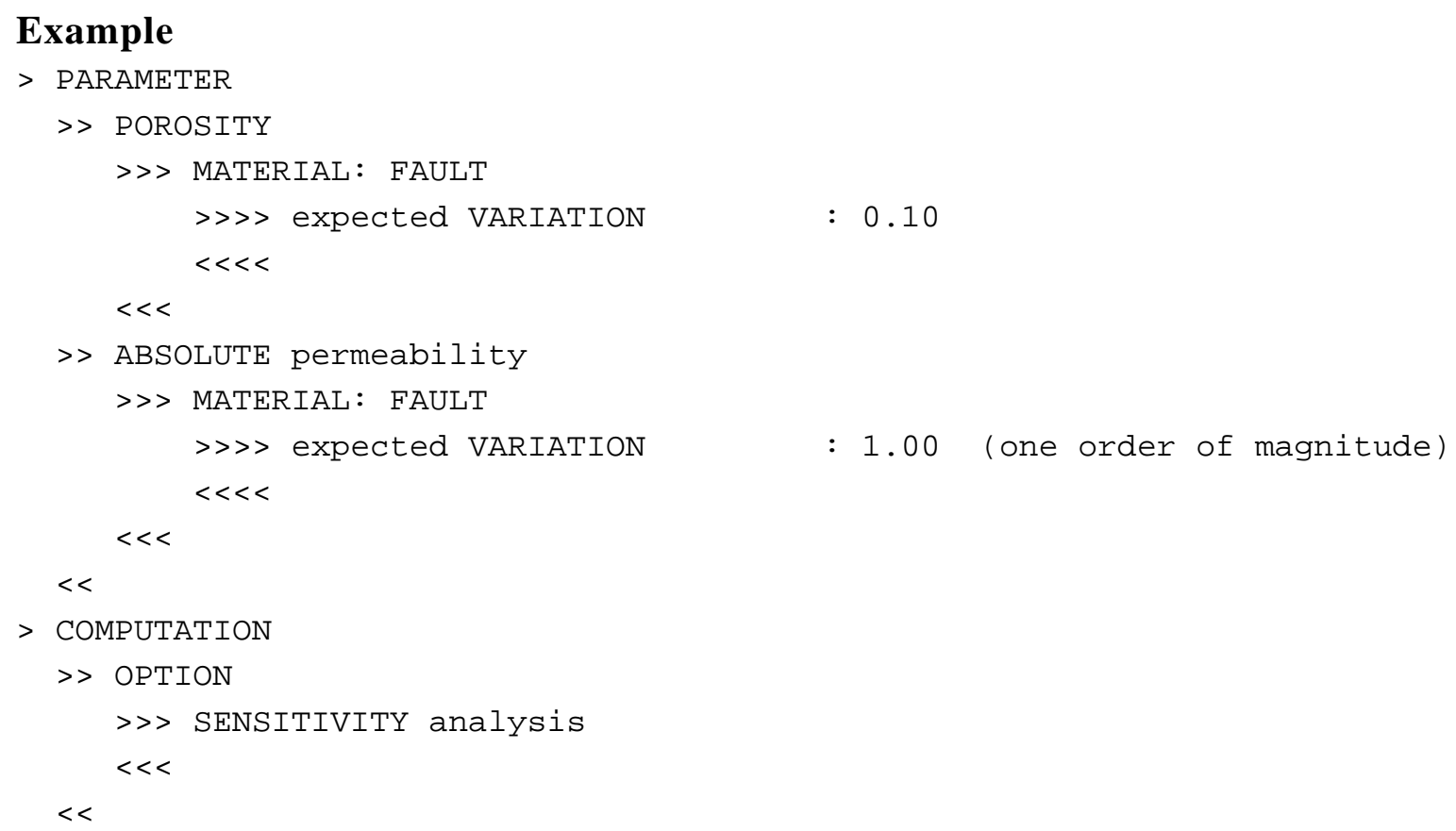

See Also

>>> DEVIATION ( $p$ ) 


\section{Command}

>> VERSION

\section{Parent Command}

>> OUTPUT

\section{Subcommand}

$-$

\section{Description}

This command prints version control statements at the end of the iTOUGH2 output file. The same information is always written to the *.msg file. If making code modifications it is strongly suggested to update the version control statement at the beginning of each subroutine or function.

\section{Example}

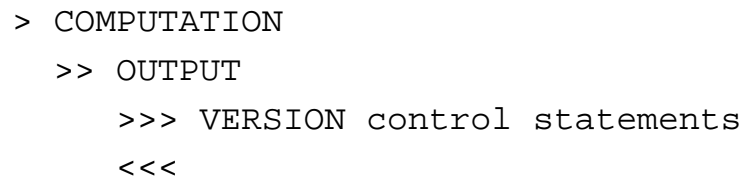

On output:

\begin{tabular}{|c|c|c|c|c|}
\hline PROGRAM & VERSON & J DATE & & COMMENT \\
\hline iTOUGH2 & & Current ver & sion & iTOUGH2 V4.0 (January 19, 1999) \\
\hline iTOUGH & 1.0 & 1 AUGUST & 1992 & iTOUGH User's Guide, Version 1.0, Report NIB 92-99 \\
\hline iTOUGH2 & 2.2 & 1 FEBRUARY & 1994 & iTOUGH2 User's Guide, Version 2.2, Report LBL-34581 \\
\hline iTOUGH2 & 3.0 & 12 JULY & 1996 & YMP Software qualification, Report LBNL-39489 \\
\hline iTOUGH2 & 3.1 & 1 APRIL & 1997 & iToUGH2 Command Reference, V3.1, Report LBNL-40041 \\
\hline iTOUGH2 & 3.2 & 30 JUNE & 1998 & YMP Software qualification, Report LBNL-42002 \\
\hline iTOUGH2 & 3.3 & 1 OCTOBER & 1998 & Parallelization using PVM, Report LBNL-42261 \\
\hline iTOUGH2 & 4.0 & 19 JANUARY & 1999 & Report LBNL-40040 \\
\hline WHATCOM & 1.0 & 10 AUGUST & 1993 & \#35: Q: WHAT COMPUTER IS USED? A: SUN \\
\hline CALLSIG & 1.0 & 5 DECEMBER & 1995 & \#112: SIGNAL HANDLER \\
\hline CPUSEC & 1.0 & 10 AUGUST & 1993 & \#--: RETURNS CPU-TIME (VERSION SUN) \\
\hline OPENFILE & 2.5 & 4 JUNE & 1996 & \#31: OPENS MOST OF THE FILES \\
\hline LENOS & 1.0 & $1 \mathrm{MARCH}$ & 1992 & \#28: RETURNS LENGTH OF LINE \\
\hline PREC & 1.0 & 1 AUGUST & 1992 & \#86: CALCULATE MACHINE DEPENDENT CONSTANTS \\
\hline ITHEADER & 3.2 & 27 MAY & 1998 & \#29: PRINTS ITOUGH2 HEADER \\
\hline DAYTIM & 1.0 & 10 AUGUST & 1993 & \#32: RETURNS DATE AND TIME (VERSION SUN) \\
\hline THEADER & 3.2 & 27 MAY & 1998 & \#30: PRINTS TOUGH2 HEADER \\
\hline INPUT & 4.0 & 19 JANUARY & 1999 & READ ALL DATA PROVIDED THROUGH FILE *INPUT*, + IFS \\
\hline MESHM & 1.0 & 24 MAY & 1990 & EXECUTIVE ROUTINE FOR INTERNAL MESH GENERATION \\
\hline MINC & 1.0 & 22 JANUARY & 1990 & "SECONDARY" FRACTURED-POROUS MEDIUM MESH \\
\hline
\end{tabular}

See Also

>> UPDATE 


\section{Command}

>> VOLUME (phase_name/PHASE: iphase) (CHANGE)

\section{Parent Command}

$>$ OBSERVATION

\section{Subcommand}

$\gg>$ MATERIAL

$>>$ MODEL

\section{Description}

This command selects as an observation type the total volume of phase iphase. This observation type refers either to the entire model domain (command >>> MODEL, see also Section A8), or to the subdomain specified by a list of rock types (command >> MATERIAL).

The phase name phase_name or phase number iphase, which depend on the EOS module being used, are listed in the iTOUGH2 header. They can be specified either on the command line or using the subcommand $>>>>$ PHASE.

If keyword CHANGE is present, the change of the total volume since data initialization is computed. This option can be used, for example, to calculate the cumulative volumetric liquid flow which is equivalent to the change of the total gas volume in place.

\section{Example}

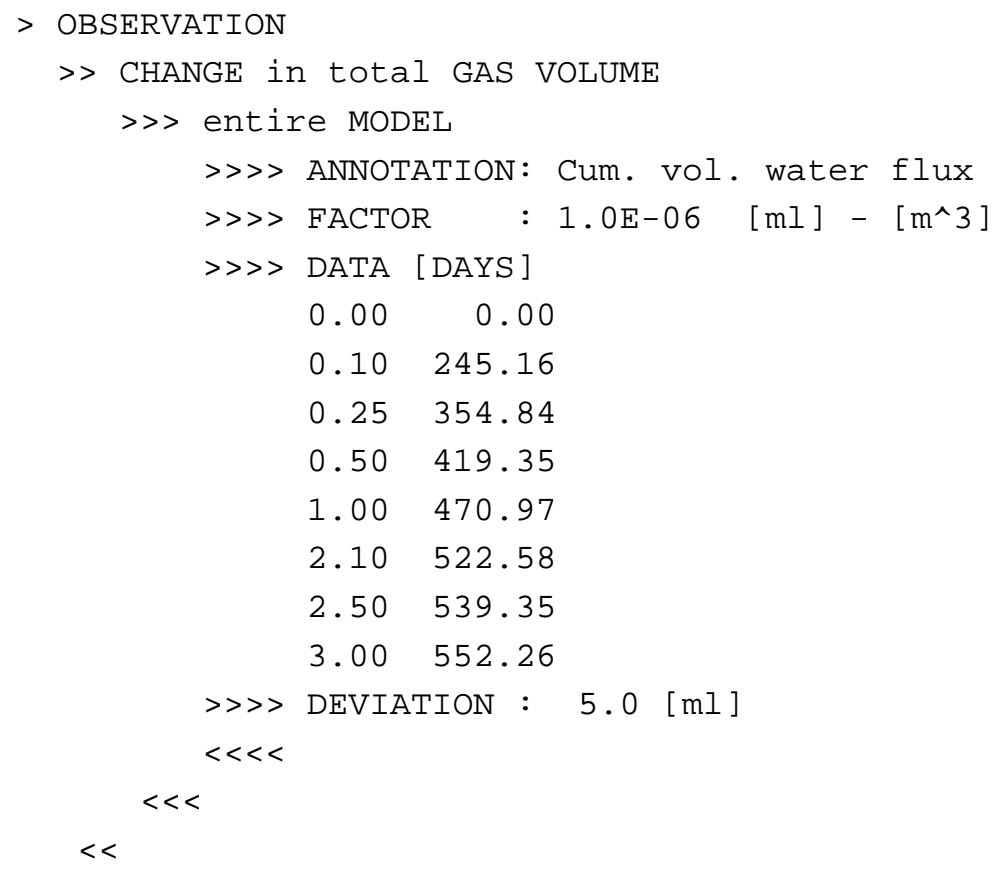

See Also 


\section{Command}

$>>$ WARNING

\section{Parent Command}

>> CONVERGE

\section{Subcommand}

$-$

\section{Description}

iTOUGH2 checks the consistency of the TOUGH2 and iTOUGH2 input, printing error and warning messages to the iTOUGH2 output file. The program stops if an error or warning is encountered. Command $>>>$ WARNING makes iTOUGH 2 continue despite the occurrence of a warning message.

It is strongly suggested to resolve all warning messages before continuing. Warning messages should only be ignored if the reason for message is completely understood and deemed harmless.

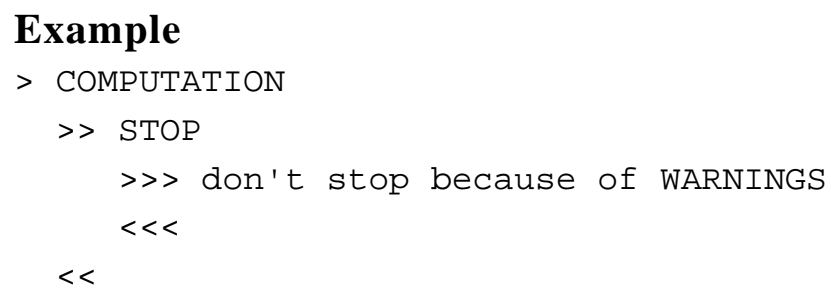

See Also

$>>$ INPUT 
Command

>>> WEIGHT: 1/sigma

Parent Command

all third-level commands in block > PARAMETER and > OBSERVATION

Subcommand

Description

(see $>>>>$ DEVIATION $(\mathrm{p} / \mathrm{o})$ )

Example

$($ see $>>>$ DEVIATION $(\mathrm{p} / \mathrm{o}))$

See Also

$>>>$ DEVIATION ( $\mathrm{p} / \mathrm{o})$ 


\section{Command}

>>> WINDOW: time_A time_B (time_unit)

Parent Command

all third-level commands in block > OBSERVATION

\section{Subcommand}

\section{Description}

The times at which the observed and calculated system response are compared are referred to as calibration times. They are specified using command >> TIME. The calibration times are defined globally, i.e., there is only one set of calibration times and it is applied to all data sets. Calibration times and observation times may be different; observed data are linearly interpolated between adjacent data points to obtain a value at the calibration time.

In the standard case, the first data point is observed at or before the first calibration time, and the last data point is observed at or after the last calibration time. This configuration ensures that a value for calibration is available or can be interpolated at every calibration point. The standard situation is sketched in Figure (a) below, assuming that equally spaced calibration times (dashed lines) have been defined, i.e., using the following command line:

\section{>> TIMES: 7 EQUALLY SPACED}

If, for some reason, only a subset of the available data can be used for calibration as shown in Figure (b) below, the user must specify the time window between which data should be processed: time_A and time_B are the lower and upper bounds of the window. Only one time window may be specified per data set. If more than one time window is required, new data sets containing the same data must be generated and different time windows specified for each data set. The time units of time_A and time_B can be specified using one of the time_unit keywords.

If a data set does not contain data that extend over the entire range of calibration times, iTOUGH2 automatically adjusts the time window to coincide with the first and last data point as shown in Figure (c). A warning message is printed, however, which can be avoided by explicitly specify the time window.

If the specified time window is larger than the available data set, the value of the first and last data point is horizontally extrapolated to time_A and time_B, respectively, as indicated in Figure (d).

If time is shifted, the time window must be given with reference to the time system of the data, if command $>>>$ SHIFT TIME: tshift appears before command >>>> WINDOW. Conversely, the window must be given in the shifted time system, if the shift command appears after command $>>>$ WINDOW. 

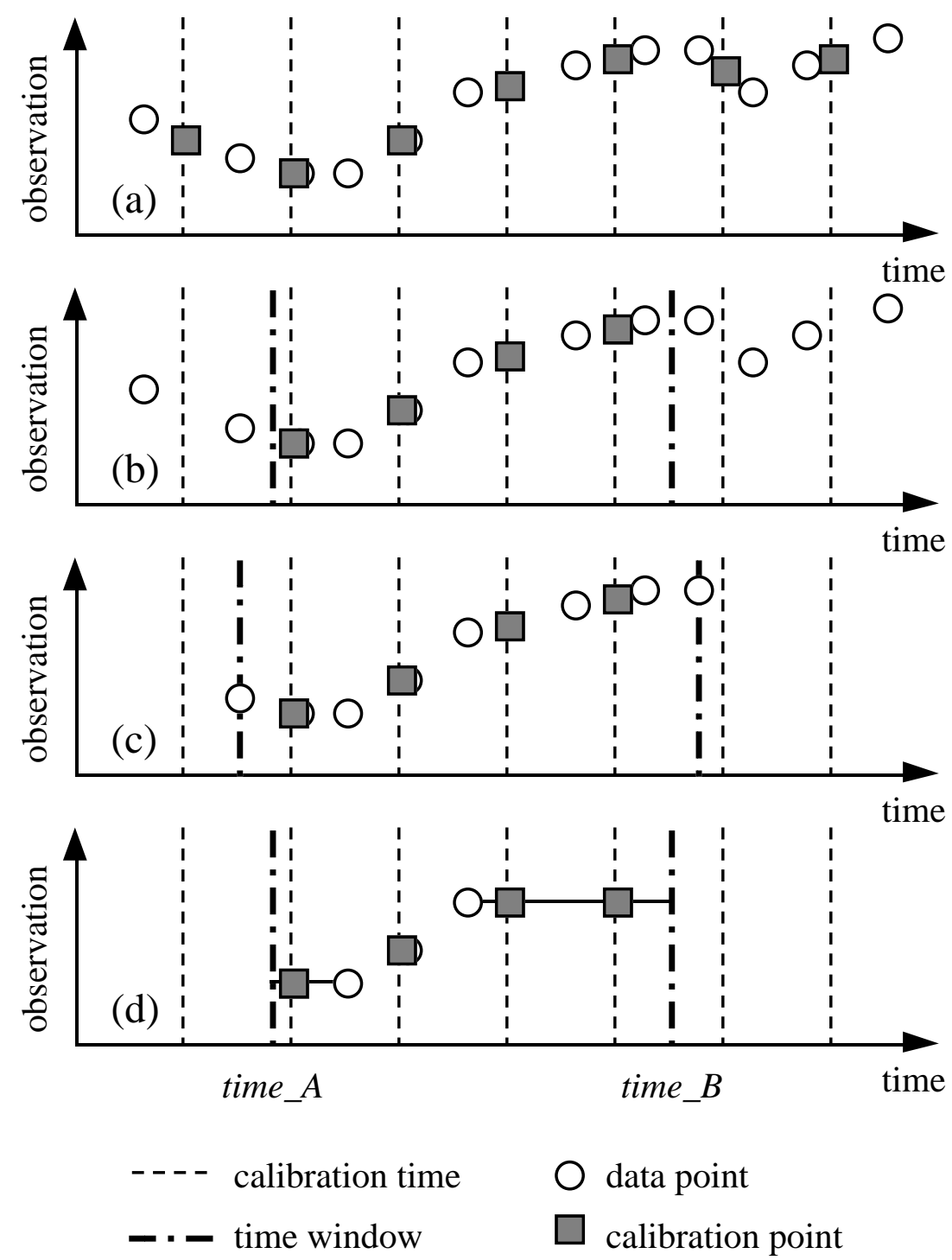

\section{Example}

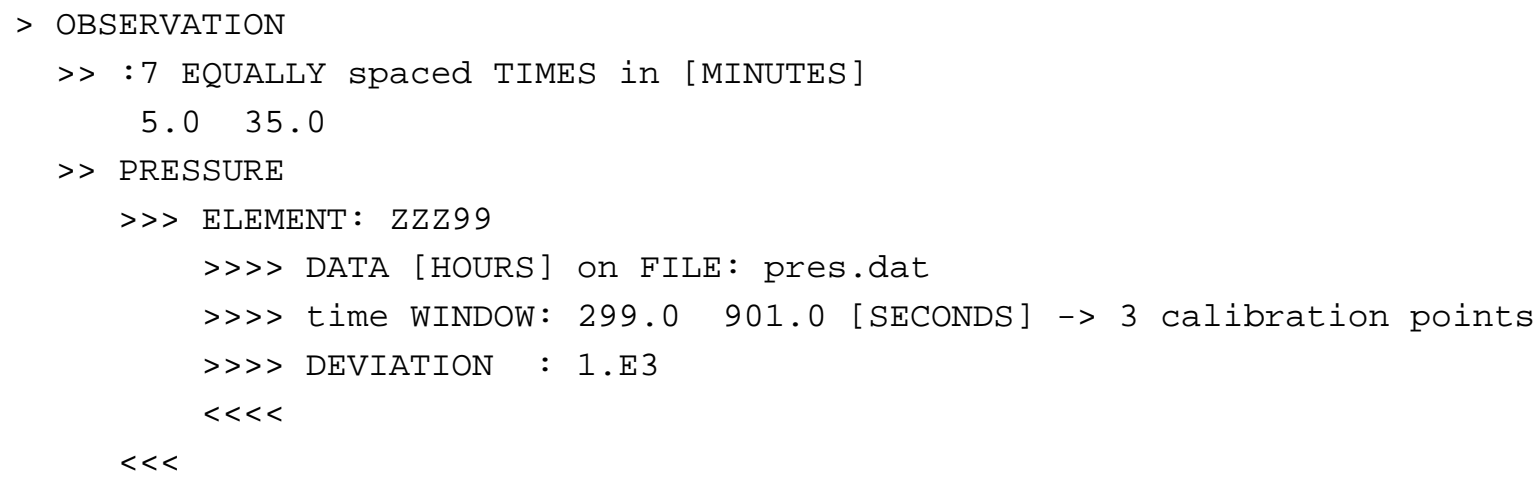

See Also

$>$ TIME, $>>>>$ SHIFT 


\section{ACKNOWLEDGMENT}

iTOUGH2 (up to version 1.1) was developed at the Laboratory of Hydraulics, Hydrology, and Glaciology (VAW) of the Swiss Federal Institute of Technology (ETH), Zürich, Switzerland, in collaboration with the Swiss National Cooperative for the Disposal of Radioactive Waste (Nagra), Wettingen, Switzerland. Subsequent versions were supported, in part, by the Assistant Secretary for Energy Efficiency and Renewable Energy, Office of Geothermal Technologies, of the U.S. Department of Energy, under Contract No. DEAC03-76SF00098, and by a grant from the Swiss National Cooperative for the Disposal of Radioactive Waste (Nagra), Wettingen, Switzerland.

Many people have made valuable suggestions and contributions during the long and ongoing development of iTOUGH2. I would like to thank J. Trösch, U. Kuhlmann (VAW) and S. Vomvoris (Nagra) for their support during the early stages of the project. Even though the main avenues of iTOUGH2 applications have been used extensively, there are many combinations of options that remain to be tested. Many errors, flaws, and inconsistencies have been detected by those who tried to apply earlier versions of iTOUGH2, and many new capabilities have been proposed. iTOUGH 2 has greatly benefited from this phase of beta-testing. I would like to acknowledge the contributions of R. Senger (INTERA/CPC, Baden, Switzerland), S. Webb (Sandia National Laboratories, Albuquerque), S. White (Industrial Research Ltd., New Zealand), E. Roeder (Clemson University), T. Sonnenborg (Technical University of Denmark), A. Battistelli (Aquater S.p.A., Italy), as well as C. Doughty, R. Ahlers, and F. Hale (LBNL). Special thanks are due to D. Bullivant and M. O'Sullivan (University of Auckland, New Zealand) who helped me restructure and streamline the coding of iTOUGH2, and to A. James and C. Doughty (LBNL) who tested some of the less frequently used options, and who thoroughly reviewed Chapter 4 of this report, making many of the command descriptions less cryptic. R. Falta (Clemson University) gave me the opportunity to write an adaptation of iTOUGH2 for PCs. C. Doughty and A. Simmons (LBNL) are thanked for their careful reviews.

If iTOUGH2 turns out to be a useful tool, this is mainly due to its powerful engine which is the simulator TOUGH2. I thank K. Pruess for his continuous support. 


\section{NOMENCLATURE}

$\alpha \quad$ level of significance; $(1-\alpha)$ is confidence level

$\mathbf{C}_{p p} \quad n \times n$ covariance matrix of estimated parameters; $\mathbf{C}_{p p}=s_{0}^{2}\left(\mathbf{J}^{T} \mathbf{V}_{z z}^{-1} \mathbf{J}\right)^{-1}$

$\mathbf{C}_{z z} \quad m \times m$ a priori covariance matrix of measurement error,

$\mathbf{C}_{\hat{z} \hat{z}} \quad m \times m$ a posteriori covariance matrix of predicted system response, $\mathbf{C}_{\hat{z} \hat{z}}=\mathbf{J} \mathbf{C}_{p p} \mathbf{J}^{T}$

J $\quad m \times n$ Jacobian matrix with elements $J_{i j}=\partial z_{i} / \partial p_{j}$

$\lambda \quad$ Levenberg parameter

$m \quad$ number of calibration points

$n \quad$ number of parameters

$v \quad$ Marquardt parameter

p parameter vector (dimension $n$ )

p* prior information vector (dimension $n$ )

$\mathbf{p}_{0} \quad$ vector of initial parameter guesses (dimension $n$ )

$\mathbf{r}$ residual vector (dimension $m$ ) with elements $r_{i}=z_{i} *-z_{i}(\mathbf{p})$

$\sigma_{i}^{2} \quad$ a priori error variance

$s_{0}^{2} \quad$ a posteriori or estimated error variance; $s_{0}^{2}=\frac{\mathbf{r}^{T} \mathbf{V}_{z z}^{-1} \mathbf{r}}{m-n}$

$S \quad$ objective function, e.g., sum of squared weighted residuals

$t \quad$ time

$\mathbf{V}_{z z}^{-1} \quad m \times m$ weighting matrix, where $\mathbf{C}_{z z}=\sigma_{0}^{2} \cdot \mathbf{V}_{z z}$

$\mathbf{x} \quad$ coordinate vector (dimension 3 )

$X \quad$ TOUGH2 input parameter

$X_{0} \quad$ initial guess of TOUGH2 input parameter

$\mathbf{z} \quad$ vector of observable variables (dimension $m$ )

$\mathbf{z}^{*} \quad$ measurement vector (dimension $m$ ), including prior information

$\hat{\mathbf{z}} \quad$ predicted system response (dimension $m$ ) 


\section{REFERENCES}

Doughty, C. A., Estimation of Hydrologic Parameters of Heterogeneous Geologic Media with an Inverse Method Based on Iterated Function Systems, Report LBL-38136, Lawrence Berkeley Laboratory, Berkeley, Calif., 1995.

Falta, R. W., K. Pruess, S. Finsterle, and A. Battistelli, T2VOC User's Guide, Report LBL-36400, Lawrence Berkeley Laboratory, Berkeley, Calif., 1995.

Finsterle, S., Parallelization of iTOUGH2 Using PVM, Report LBNL-42261, Lawrence Berkeley National Laboratory, Berkeley, Calif., 1998.

Finsterle, S., iTOUGH2 User's Guide, Report LBNL-40401, Lawrence Berkeley National Laboratory, Berkeley, Calif., 1999a.

Finsterle, S., iTOUGH2 Command Reference, Report LBNL-40401 (Updated reprint), Lawrence Berkeley National Laboratory, Berkeley, Calif., 1999 b.

Finsterle, S., iTOUGH2 Sample Problems, Report LBNL-40402 (Updated reprint), Lawrence Berkeley National Laboratory, Berkeley, Calif., 1999c.

Finsterle, S., G. J. Moridis, and K. Pruess, A TOUGH2 Equation-of-State Module for the Simulation of Two-Phase Flow of Air, Water, and a Miscible Gelling Liquid, Report LBL-36086, Lawrence Berkeley Laboratory, Berkeley, Calif., 1994.

Geist, A., A. Beguelin, J. Dongarra, W. Jiang, R. Manchek, and V. Sunderam, PVM: Parallel Virtual Machine-A User's Guide and Tutorial for Networked Parallel Computing, MIT Press, Cambridge, 1994.

(This book can be viewed at URL http://www.netlib.org/pvm3/book/pvm-book.html; a PostScript file can be retrieved from the anonymous ftp server netlib2.cs.utk.edu, directory pvm3/book, file pvm-book.ps)

Haining, R., Spatial Data Analysis in the Social and Environmental Sciences, Cambridge University Press, Cambridge, 1990.

Kitterød, N.-O., and L. Gottschalk, Simulation of normal distributed smooth fields by Karhunen-Loéve expansion in combination with kriging, Stochastic Hydrology and Hydraulics, 11, 459-482, 1997.

Levenberg, K., A method for the solution of certain nonlinear problems in least squares, Quart. Appl. Math., 2, 164-168, 1944.

Liu, H.-H., C. Doughty, and G. S. Bodvarsson, An active fracture model for unsaturated flow and transport in fractured rocks, Water Resour. Res., 34(10), 2633-2646, 1998. 
Luckner, L., M. Th. van Genuchten, and D. Nielsen, A consistent set of parametric models for the two-phase flow of immiscible fluids in the subsurface, Water Resour. Res., 25(10), 2187-2193, 1989.

Marquardt, D.W., An algorithm for least squares estimation of nonlinear parameters, SIAM J. Appl. Math., 11, 431-441, 1963.

Moridis, G. J., and K. Pruess, Flow and Transport Simulations Using T2CG1, a Package of Conjugate Gradient Solvers for the TOUGH2 Family of Codes, Report LBL-36235, Lawrence Berkeley Laboratory, Berkeley, Calif., 1995.

Pruess, K., TOUGH User's Guide, Report NUREG/CR-4645, Nuclear Regulatory Commission (also Report LBL-20700, Lawrence Berkeley Laboratory, Berkeley, Calif.), 1987.

Pruess, K., TOUGH2-A General-Purpose Numerical Simulator for Multiphase Fluid and Heat Flow, Report LBL-29400, Lawrence Berkeley Laboratory, Berkeley, Calif., $1991 \mathrm{a}$.

Pruess, K., EOS7, an Equation-of-State Module for the TOUGH2 Simulator for Two-Phase Flow of Saline Water and Air, Report LBL-31114, Lawrence Berkeley Laboratory, Berkeley, Calif., 1991b. 


\section{APPENDIX A: TOUGH2 Enhancements}

\section{A 1. Introduction}

The core of iTOUGH 2 contains slightly modified versions of TOUGH 2 modules. Most code modifications are editorial and do not affect the simulation results. As a result, standard TOUGH2 input files can be used in iTOUGH2, and identical results are obtained if iTOUGH2 is run in forward mode. However, a number of minor modifications have been made as described in the following sections. These modifications should also be made in new modules added to iTOUGH2.

\section{A 2. Printout Control (KDATA)}

In standard TOUGH2, printout of simulation results is controlled by variables KDATA and $M C Y P R$ in block PARAM.1, as well as by the additional printout times TIS given in block TIMES. In iTOUGH2, the calibration times are also stored in array TIS which means that at each calibration time the amount of printout specified by variable KDATA is written to the output file. This may make the TOUGH2 output file extremely long, and requires unnecessary CPU time for disk writing, since the TOUGH2 output file is overwritten each time a new TOUGH2 simulation is initiated by iTOUGH2.

If a negative number is specified for variable $K D A T A$, the amount of printout is reduced (see Table A2.1), saving both disk space and CPU time. Recall that printout at the calibration points is always written to the iTOUGH2 plot file (see command $>>>$ FORMAT). Also, if a TOUGH 2 run is terminated due to a convergence failure or using command $\mathrm{kit}$, the full output is automatically generated for the last time step.

Full output is always provided for the times specified in the TOUGH2 block TIMES; the amount of printout is given by the absolute value of $K D A T A$. The times provided in TOUGH2 block TIMES can be in arbitrary order; they are sorted internally.

Table A2. Amount of Printout as a Function of Variable KDATA

\begin{tabular}{ccccccc}
\hline-3 & -2 & -1 & Printout of ... & 1 & 2 & 3 \\
\hline- & + & + & volume and mass balance & + & + & + \\
- & - & + & generation rates & + & + & + \\
- & - & - & most important variables & + & + & + \\
- & - & - & fluxes and velocities & - & + & + \\
- & - & - & primary variables & - & - & + \\
\hline
\end{tabular}




\section{A3. Secondary Mesh (ELEM2, CONN2)}

This enhancement was introduced especially for the estimation of a skin radius (see command >> SKIN) or a MINC parameter (see command >> MINC), both requiring that a new TOUGH 2 mesh is generated automatically each iteration without user interference. In order to achieve this, a primary mesh must be generated using the MESHMAKER utility. Elements and connections of this primary mesh can then be overwritten by a secondary mesh provided through blocks ELEM2 and CONN2 which have the same format as blocks ELEME and CONNE, respectively. The names of the elements and connections to be modified by the secondary mesh must be identical with the corresponding ones of the primary mesh. If a secondary mesh is specified, both blocks, ELEM2 and CONN2, must be given; either of the two keywords may be followed by an empty line to indicate that no modification is to be made.

Figure A3.1 illustrates an application. A radial mesh is generated using MESHMAKER for simulating a pump test. In block ELEM2, the volume of the first grid block is changed to represent the actual interval volume. In block CONN2, the nodal distance from the first element to the interface is reduced to a very small number as usually done for connections to boundary elements. These last two modifications, which are usually made by editing the mesh file, are now automatically performed whenever a new mesh is generated during an iTOUGH2 run for the estimation of the skin radius.

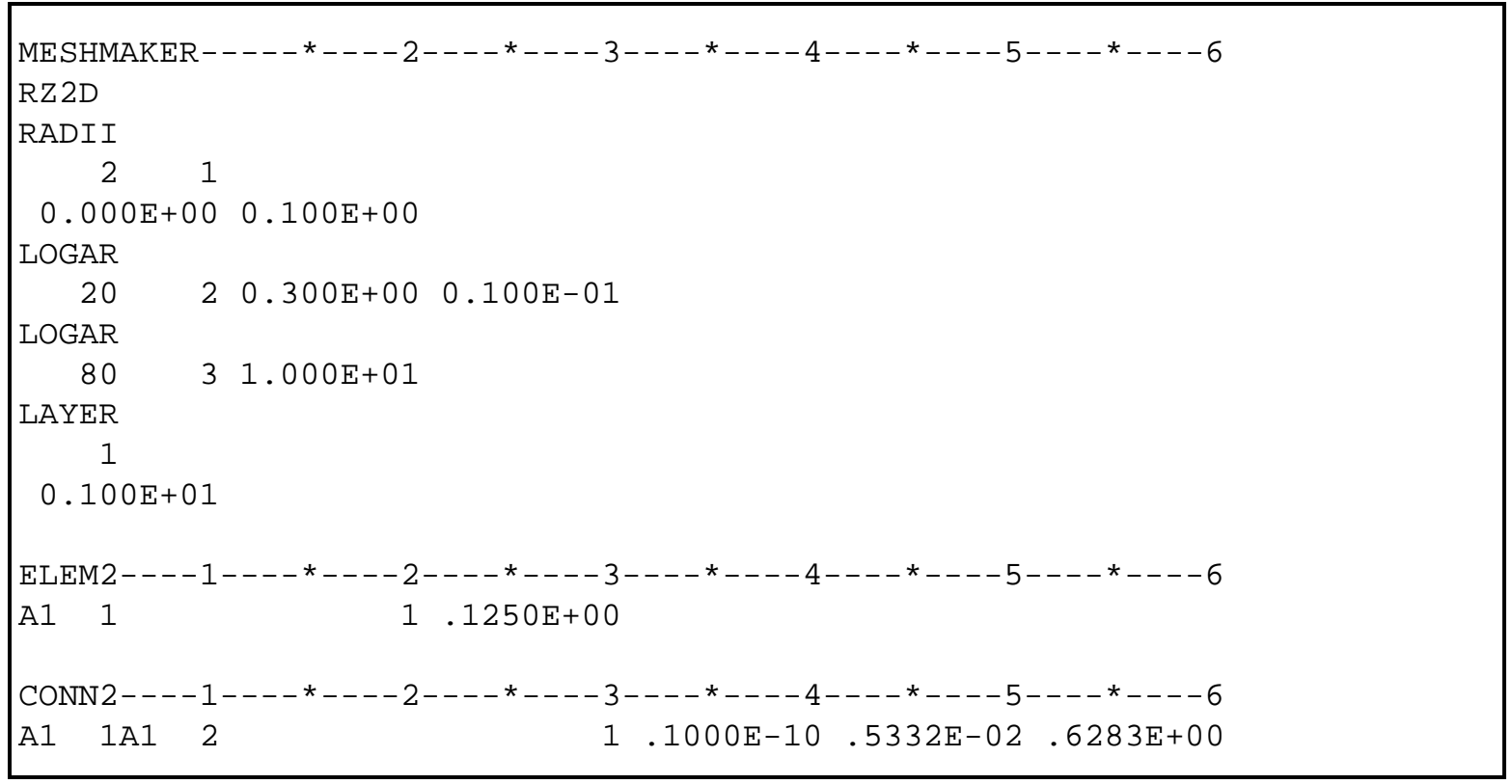

Figure A3.1. Primary and secondary mesh generation. 


\section{A 4. Element-by-Element Permeability}

Heterogeneity is introduced into TOUGH2 models by assigning a certain rock type, for which material properties are defined in block ROCKS, to each grid block. The maximum number of rock types one can specify is given by variable MAXROC (see file maxsize.inc). For high resolution simulations of permeability heterogeneity, it is inconvenient to specify hundreds of rock types, one for each grid block. TOUGH2 has been extended so that grid block permeabilities or permeability modifiers can be directly specified in block ELEME, columns 41-50. If a positive value is given, it is interpreted as absolute permeability; if a negative value is provided, it is interpreted as a permeability modifier, i.e., a factor with which the absolute permeability specified in block ROCKS is multiplied. The anisotropy ratio as given in block ROCKS is preserved in both cases. Alternatively, the same information can be provided through block INCON, columns 31-40. Four additional parameters specific to a grid block can be provided in block INCON, columns 41-80.

Using a permeability modifier instead of permeability itself has the advantage that the mean of the permeability field can easily be changed (or estimated) by adjusting parameter PER (ISOT) in block ROCKS.

The permeability or permeability modifiers are stored in array $\operatorname{USERX}(1, N)$, where $N$ is the grid block number, and additional parameters are stored in $\operatorname{USERX}(i, N)$ for $i=2 \ldots 5$. The second parameter, $\operatorname{USERX}(2, N)$, is reserved for a factor scaling capillary strength (see Appendix A6 for details). To write element-by-element parameters to file $S A V E$, set $M O P(13)$ to 1 .

\section{A 5. User-Specified Boundary Conditions}

In TOUGH2, Neumann boundary conditions are specified by introducing sinks and sources in block GENER. Dirichlet boundary conditions can be implemented by assigning very large volumes to grid blocks adjacent to the boundary so that the thermodynamic conditions in those elements do not change from fluid and heat exchange with finite-size grid blocks in the model domain.

Prescribed, but time-varying boundary conditions can be implemented by specifying appropriate (large) sinks and sources in grid blocks having a very large volume. For simple step changes, however, iTOUGH2 offers an alternative option (see command >> RESTART TIME). A third possibility is described in this section. The user can provide values of the primary variables for selected elements as a function of time. The function has to be programmed into subroutine USERBC, which can be found in file it2user.f. In subroutine USERBC, the user has the possibility to provide the value of one or more primary variables for selected elements as a function of time. For example, these values can be calculated internally or read from a file. The header of subroutine USERBC is shown in Figure A5.1. The element number $\mathrm{N}$ or element name CELEM can be used to identify the boundary grid block. The user is supposed to return a value for one or several of the primary variables 
through array $\mathrm{X}$. In the example given below, a table of time versus pressure data is read from file atm_pres.dat and assigned to element 'ATM O' using the linear interpolation function INTERP1. Note that either the full path to file atm_pres.dat must be given, or the file must be copied to the temporary directory using option $-\mathrm{fi}$ filename.

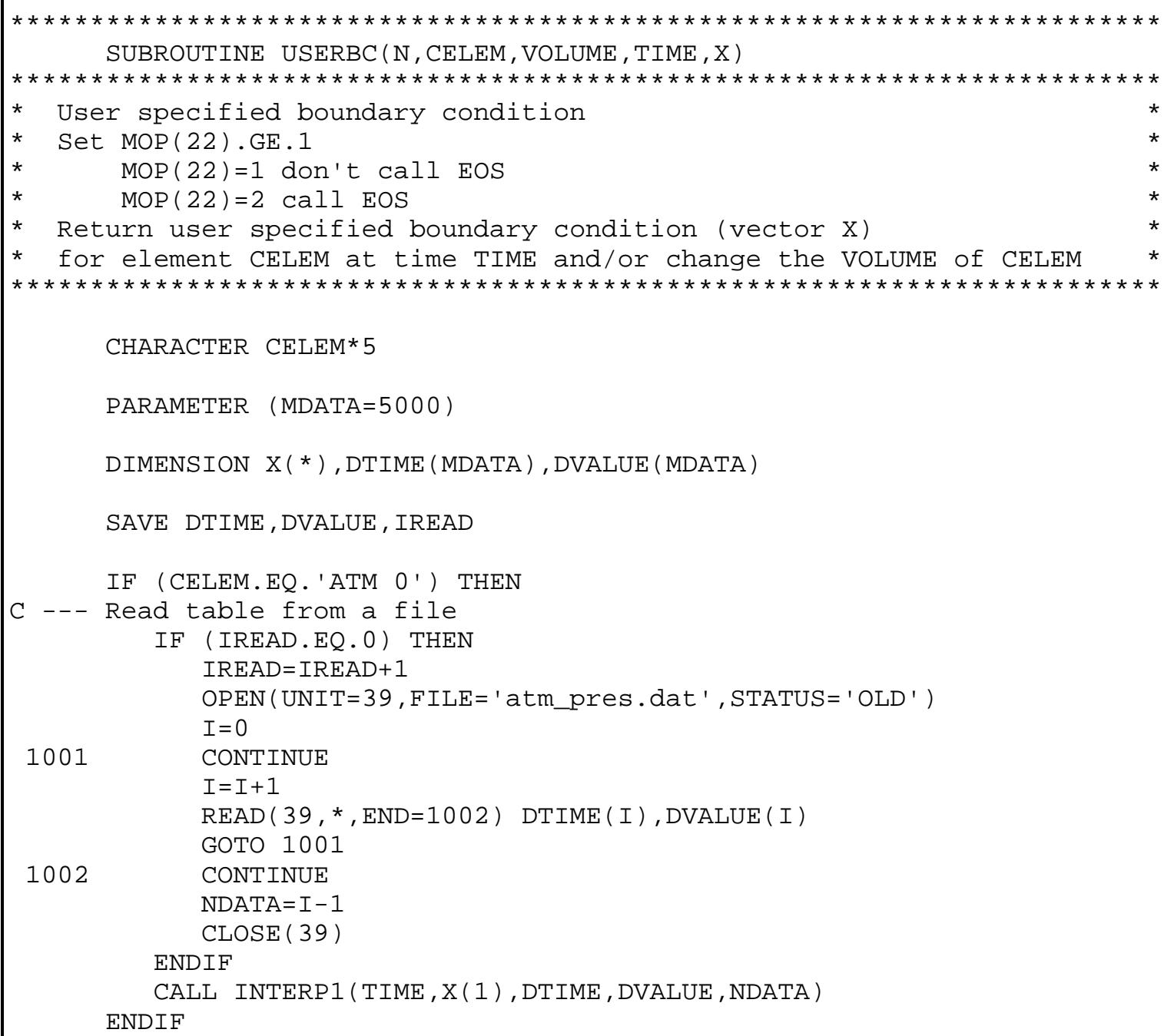

Figure A5.2. Subroutine USERBC for specifying time-dependent boundary conditions.

Subroutine USERBC is called only if $M O P$ (22) is either 1 or 2. If $M O P(22)$ is 2, the EOS module is called after completion of a time step to ensure that the user-specified thermodynamic conditions are updated. If MOP (22) is 1, subroutine USERBC is called, but no additional call to subroutine EOS is made. This allows one to make time-dependent changes to TOUGH2 variables that are not primary variables, and that do not require a recalculation of the thermodynamic state. 


\section{A 6. Relative Permeability and Capillary Pressure Functions}

Subroutines RELP and PCAP provide the relative permeability and capillary pressure functions, respectively (see Pruess [1987]). They are frequently modified to accommodate particular needs. The user should therefore carefully check the functional form and required parameters before selecting a certain curve through variable $I R P$ and $I C P$, respectively (see also command >> CHARACTERISTIC).

The functions provided in this version include the three-phase curves used by the T2VOC module [Falta et al., 1995]. Additional functions are provided as described in the following subsections.

\section{A6.1 Modified Brooks-Corey model}

A modified version of the Brooks-Corey model [Luckner et al., 1989] has been implemented. In order to prevent the capillary pressure from decreasing towards negative infinity as the effective saturation approaches zero, a linear function is used for saturations $S_{l}$ below a certain value $\left(S_{l r}+\varepsilon\right)$, where $\varepsilon$ is a small number. The slope of the linear extrapolation is identical with the slope of the capillary pressure curve at $S_{l}=S_{l r}+\varepsilon$. Alternatively, the capillary pressure is prevented from becoming more negative than $-p_{c, \max } \cdot$

The modified Brooks-Corey model is invoked by setting both IRP and ICP to 10 . The model is described by the following set of equations (the input parameters are listed in Table A6.1.1):

$$
\begin{gathered}
S_{e c}=\frac{S_{l}-S_{l r c}}{1-S_{l r c}} \\
S_{e k}=\frac{S_{l}-S_{l r k}}{1-S_{l r k}-S_{g r}} \\
p_{c}=-p_{e}\left(S_{e c}\right)^{-1 / \lambda} \quad \text { for } S_{l} \geq\left(S_{l r c}+\varepsilon\right) \\
p_{c}=-p_{e}\left(\frac{\varepsilon}{1-S_{l r c}}\right)^{-1 / \lambda}-\frac{p_{e}}{\lambda}\left(\frac{\varepsilon}{1-S_{l r c}}\right)^{-\frac{1-\lambda}{\lambda}}\left(S_{l}-S_{l r c}-\varepsilon\right) \text { for } S_{l}<\left(S_{l r c}+\varepsilon\right) \\
p_{c} \geq-p_{c, \max }
\end{gathered}
$$




$$
\begin{gathered}
k_{r l}=S_{e k} \frac{2+3 \lambda}{\lambda} \\
k_{r g}=\left(1-S_{e k}\right)^{2}\left(1-S_{e k} \frac{2+\lambda}{\lambda}\right) \\
k_{r g}=1-k_{r l}
\end{gathered}
$$

Table A6.1.1. Input Parameters for Modified Brooks-Corey Model

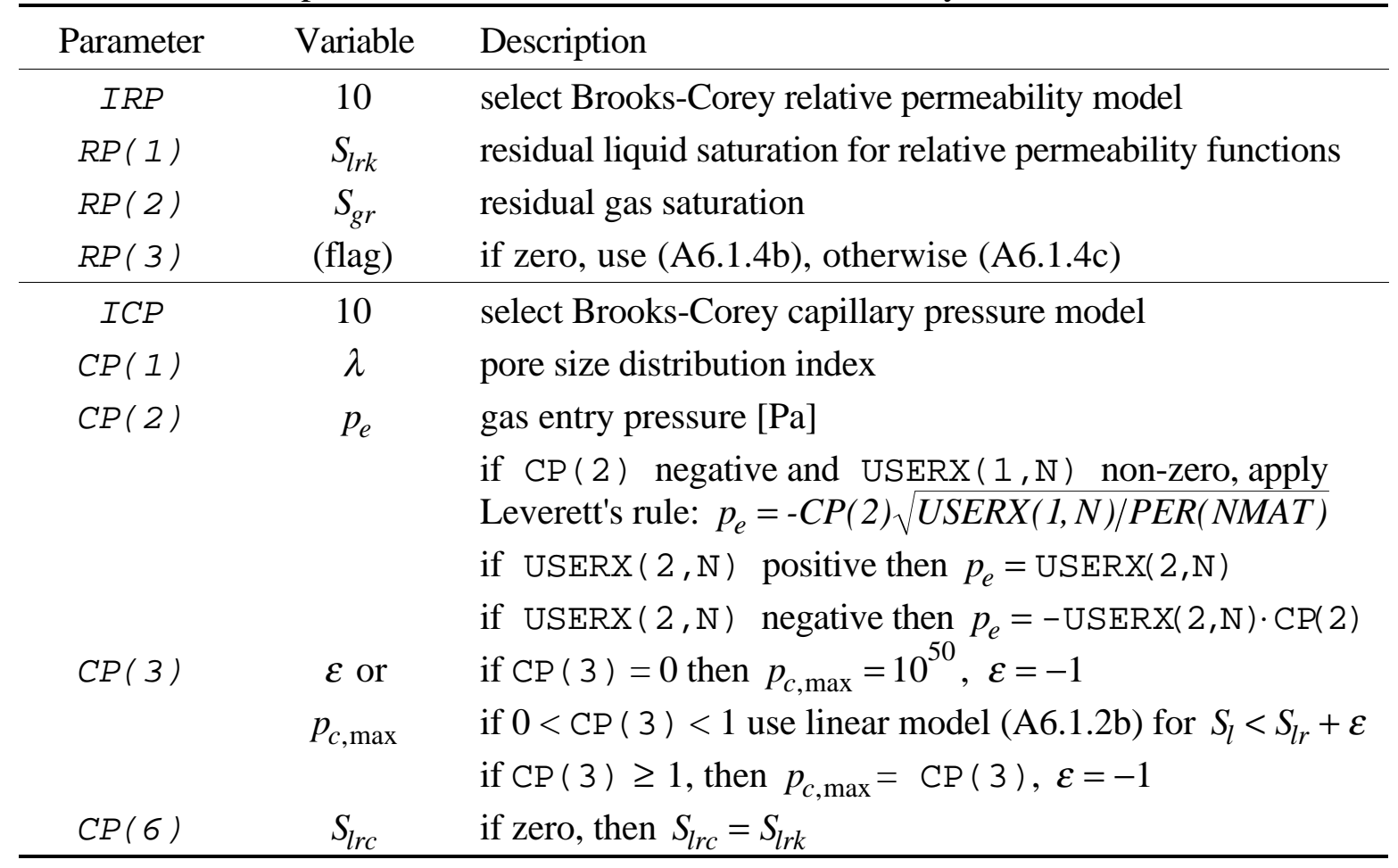
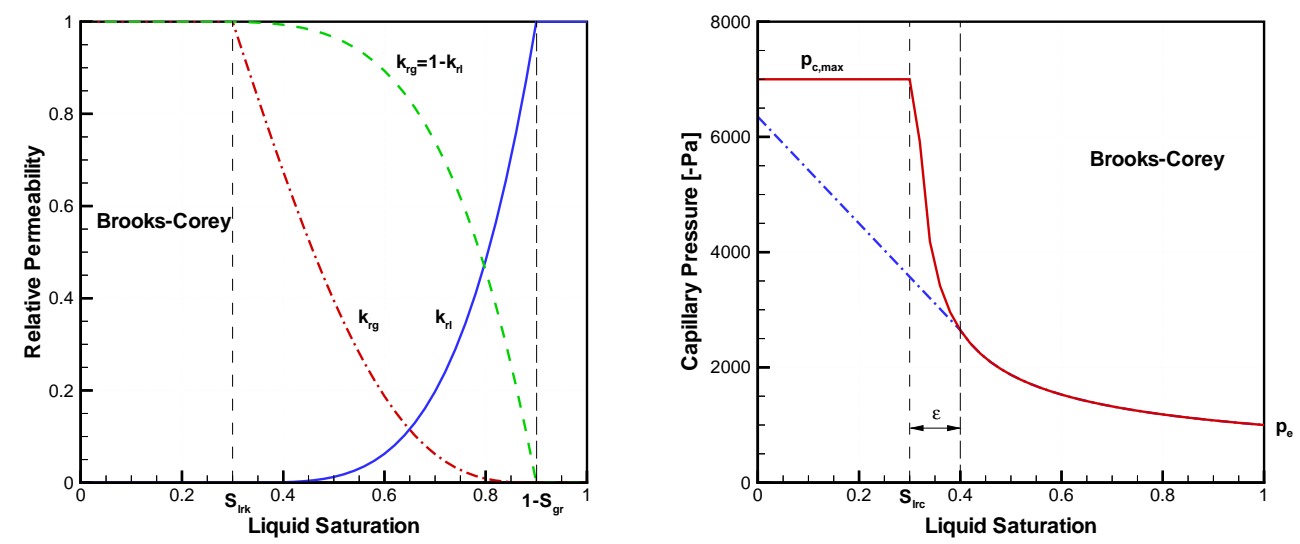

Figure A6.1.1. Modified Brooks-Corey relative permeability and capillary pressure curves. 


\section{A 6.2 Modified van Genuchten model}

A modified version of the van Genuchten model [Luckner et al., 1989] has been implemented. In order to prevent the capillary pressure from decreasing towards negative infinity as the effective saturation approaches zero, a linear function is used for saturations $S_{l}$ below a certain value $\left(S_{l r}+\varepsilon\right)$, where $\varepsilon$ is a small number. The slope of the linear extrapolation is identical with the slope of the capillary pressure curve at $S_{l}=S_{l r}+\varepsilon$. Alternatively, the capillary pressure is prevented from becoming more negative than $-p_{c, \max } \cdot$

The modified van Genuchten model is invoked by setting both IRP and ICP to 11 . The model is described by the following set of equations (the input parameters are listed in Table A6.2.1):

$$
\begin{gathered}
S_{e c}=\frac{S_{l}-S_{l r c}}{1-S_{l r c}} \\
S_{e k}=\frac{S_{l}-S_{l r k}}{1-S_{l r k}-S_{g r}} \\
p_{c}=-\frac{1}{\alpha}\left[\left(S_{e c}\right)^{-1 / m}-1\right]^{1 / n} \text { for } S_{l} \geq\left(S_{l r c}+\varepsilon\right) \\
p_{c}=-\frac{1}{\alpha}\left[\left(\frac{\varepsilon}{1-S_{l r c}}\right)^{-1 / m}-1\right]^{1 / n}+\beta\left(S_{l}-S_{l r c}-\varepsilon\right) \text { for } S_{l}<\left(S_{l r c}+\varepsilon\right) \\
\text { with } \beta=-\frac{1}{\alpha} \frac{1}{n m} \frac{1}{1-S_{l r c}}\left(S_{e c}^{-1 / m}-1\right)^{1 / n-1} S_{e c}-\left(\frac{1+m}{m}\right) \\
p_{c} \geq-p_{c, \max } \\
k_{r l}=S_{e k}^{1 / 2}\left[1-\left(1-S_{e k}{ }^{1 / m}\right)^{m}\right]^{2} \\
k_{r g}=\left(1-S_{e k}\right)^{1 / 3}\left[1-S_{e k}{ }^{1 / m}\right]^{2 m} \\
k_{r g}=1-k_{r l}
\end{gathered}
$$


Table A6.2.1. Input Parameters for Modified van Genuchten Model

\begin{tabular}{|c|c|c|}
\hline Parameter & Variable & Description \\
\hline$I R P$ & 11 & select van Genuchten relative permeability model \\
\hline$R P(1)$ & $S_{l r k}$ & residual liquid saturation for rel. perm. functions \\
\hline$R P(2)$ & $S_{g r}$ & residual gas saturation \\
\hline$R P(3)$ & (flag) & if zero, use (A6.2.4b), if non-zero, use (A6.2.4c) \\
\hline$I C P$ & 11 & select van Genuchten capillary pressure model \\
\hline$C P(1)$ & $n$ & analogous to pore size distribution index \\
\hline \multirow[t]{4}{*}{$C P(2)$} & $1 / \alpha$ & analogous to gas entry pressure $[\mathrm{Pa}]$ \\
\hline & & $\begin{array}{l}\text { if } \mathrm{CP}(2) \text { negative and } \operatorname{USERX}(1, \mathrm{~N}) \text { non-zero, apply } \\
\text { Leverett's rule: } 1 / \alpha=-C P(2) \sqrt{\operatorname{USERX}(1, N) / \operatorname{PER}(N M A T)}\end{array}$ \\
\hline & & if $\operatorname{USERX}(2, \mathrm{~N})$ positive then $1 / \alpha=\operatorname{USERX}(2, \mathrm{~N})$ \\
\hline & & if $\operatorname{USERX}(2, \mathrm{~N})$ negative then $1 / \alpha=-\operatorname{USERX}(2, \mathrm{~N}) \cdot \operatorname{CP}(2)$ \\
\hline \multirow[t]{2}{*}{$C P(3)$} & $\varepsilon$ or & if $\mathrm{CP}(3)=0$ then $p_{c, \max }=10^{50}, \varepsilon=-1$ \\
\hline & $p_{c, \max }$ & $\begin{array}{l}\text { if } 0<\mathrm{CP}(3)<1 \text { use linear model (A6.2.2b) for } S_{l}<S_{l r}+\varepsilon \\
\text { if } \mathrm{CP}(3) \geq 1 \text {, then } p_{c, \text { max }}=\mathrm{CP}(3), \varepsilon=-1\end{array}$ \\
\hline$C P(4)$ & $m$ & if zero then $m=1-1 / n$ \\
\hline$C P(6)$ & $S_{l r c}$ & if zero, then $S_{l r c}=S_{l r k}$ \\
\hline
\end{tabular}
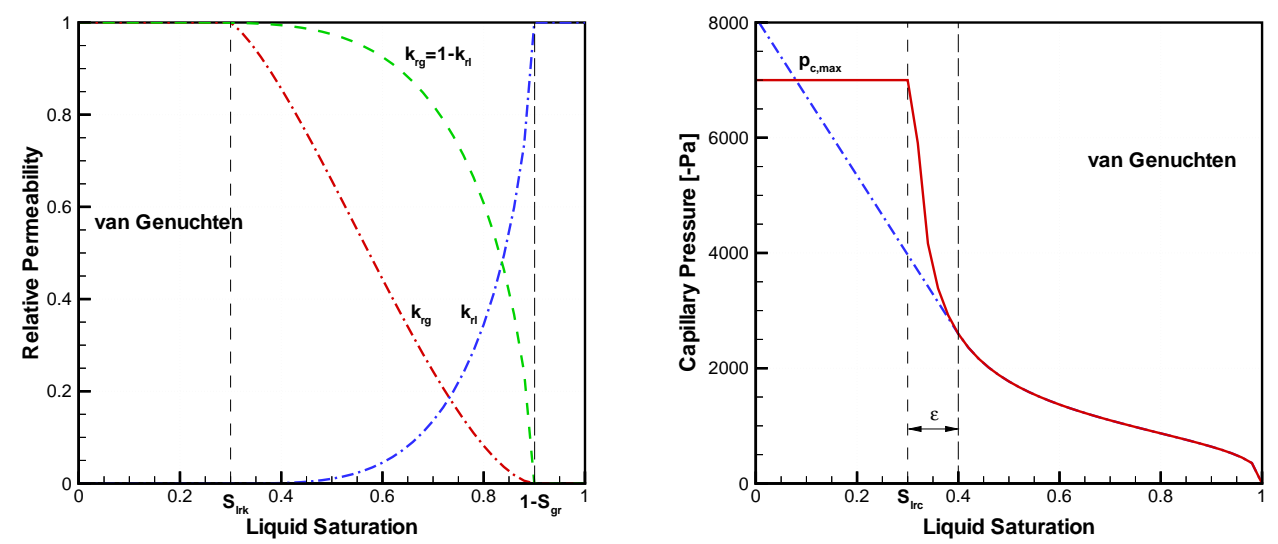

Figure A6.2.1. Modified van Genuchten relative permeability and capillary pressure curves. 


\section{A 7. Reduction of Fracture-Matrix Interface Area}

There is evidence that fracture-matrix interaction in the unsaturated zone is reduced as a result of fracture coatings as well as preferential flow in the fractures as invoked by flow instabilities (fingering) and small-scale heterogeneities. A number of options for reducing fracture-matrix interface area have been implemented for use in a dual-permeability flow simulation. Interface area reduction is applied to connections with a negative value for variable ISOT, which is provided in the CONNE block. Different modifiers are used depending on the value of ISOT and MOP (8) as summarized in Table A7.1.

Table A7.1. Option for Reducing Fracture-Matrix Interface Area

\begin{tabular}{|c|c|c|}
\hline ISOT & $\mathrm{MOP}(8)$ & Interface area reduction factor $a_{f m}$ \\
\hline positive & any & No interface area reduction, i.e., $a_{f m}=1$ \\
\hline negative & 1 & $a_{f m}=R P(6, N M A T)$ \\
\hline \multirow[t]{2}{*}{$-1,-2,-3$} & 0 & $a_{f m}=S_{\beta}$ \\
\hline & 2 & $a_{f m}=S_{\beta} \cdot R P(7, N M A T)$ \\
\hline \multirow[t]{2}{*}{$-4,-5,-6$} & 0 & $a_{f m}=k_{r \beta}$ \\
\hline & 2 & $a_{f m}=k_{r \beta} \cdot R P(7, N M A T)$ \\
\hline$-7,-8,-9$ & 0 & $a_{f m}=R P(6, N M A T)$ \\
\hline$-10,-11,-12$ & 0 & $a_{f m}=S_{e}^{1+\gamma}($ see Section A9) \\
\hline$a_{f m}$ & \multicolumn{2}{|c|}{ Fracture-matrix interface area reduction factor. } \\
\hline$S_{\beta}$ & \multicolumn{2}{|c|}{ For flow of phase $\beta$, upstream saturation of phase $\beta$. } \\
\hline & \multicolumn{2}{|c|}{ For flow of phase $\beta$, upstream relative permeability of phase $\beta$. } \\
\hline$R P(6, N M A T)^{\#}$ & \multicolumn{2}{|c|}{ 6th parameter of rel. perm. function of upstream element. } \\
\hline$R P(7, N M A T)^{\#}$ & \multicolumn{2}{|c|}{ 7th parameter of rel. perm. function of upstream element. } \\
\hline \# & \multicolumn{2}{|c|}{ If zero (i.e., not specified), reset to one. } \\
\hline
\end{tabular}

\section{A 8. Excluding Domains From Global Material Balances}

Domains with an element volume larger than $10^{20} \mathrm{~m}^{3}$ or a negative rock grain specific heat $S P H T$ or with $S P H T$ greater than $10^{4} \mathrm{~J} / \mathrm{kg}{ }^{\circ} \mathrm{C}$ are excluded from global material balance calculations. The absolute value of $S P H T$ is used in the heat balance equation. Material balances for individual rock types are available through iTOUGH2 commands >> TOTAL MASS and >>> VOLUME. 


\section{A9. Active Fracture Concept}

There is evidence that only a portion of the connected fracture network conducts water under unsaturated conditions. The fractures contributing to liquid flow are referred to as "active fractures". The Active Fracture Concept (AFC) was developed by Liu et al. [1998] to describe gravity-dominated, non-equilibrium, preferential liquid flow in fractures, which is expected to be similar to fingering in unsaturated porous media. AFC is based on the hypothesis that (1) the number of active fractures is small compared with the total number of connected fractures, (2) the number of active fractures within a grid block is large so that the continuum approach is valid, and (3) the fraction of active fractures, $f_{a}$, is related to water flux and equals one for a fully saturated system, and zero if the system is at residual saturation. The following power function of effective liquid saturation, $S_{e}$, fulfills these conditions:

$$
f_{a}=S_{e}^{\gamma}
$$

Here, $\gamma$ is a positive constant depending on properties of the fracture network, and $S_{e}$ is the effective liquid saturation given by

$$
S_{e}=\frac{S_{l}-S_{l r}}{1-S_{l r}}
$$

Capillary pressure and relative permeability functions are modified to account for the fact that the effective saturation in the active fractures, $S_{e a}$, is larger than the effective saturation of the total fracture continuum:

$$
S_{e a}=\frac{S_{e}}{f_{a}}=S_{e}^{1-\gamma}
$$

Using the van Genuchten model, capillary pressure and liquid relative permeability are given, respectively, by

$$
p_{c}=-\frac{1}{\alpha}\left[S_{e^{(\gamma-1) / m}}-1\right]^{1 / n}
$$

and

$$
k_{r l}=S_{e}^{(1+\gamma) / 2}\left\{1-\left[1-S_{e}^{(1-\gamma) / m}\right]^{m}\right\}^{2}
$$

The fracture-matrix interface area reduction factor (see Section A8) is given by

$$
a_{f m}=S_{e}^{1+\gamma}
$$

The AFC is invoked by selecting $\gamma>0$, which is provided as an additional parameter of the standard van Genuchten model $(\mathrm{ICP}=7)$ through variable $\mathrm{CP}(6, \mathrm{NMAT})$. Fracture-matrix 
interface area reduction is invoked by selecting ISOT between -10 and -12 (see Table A8.1).

\section{A 10. Free Drainage Boundary Condition}

A free drainage boundary condition for liquid flow can be implemented, in which gravity is the only driving force, i.e., (capillary) pressure pressure gradients are ignored across an interface to the boundary gridblock. This type of boundary condition comes into effect at each connection, in which one of the gridblocks belongs to rock type DRAIN. 


\section{APPENDIX B: Command Index}

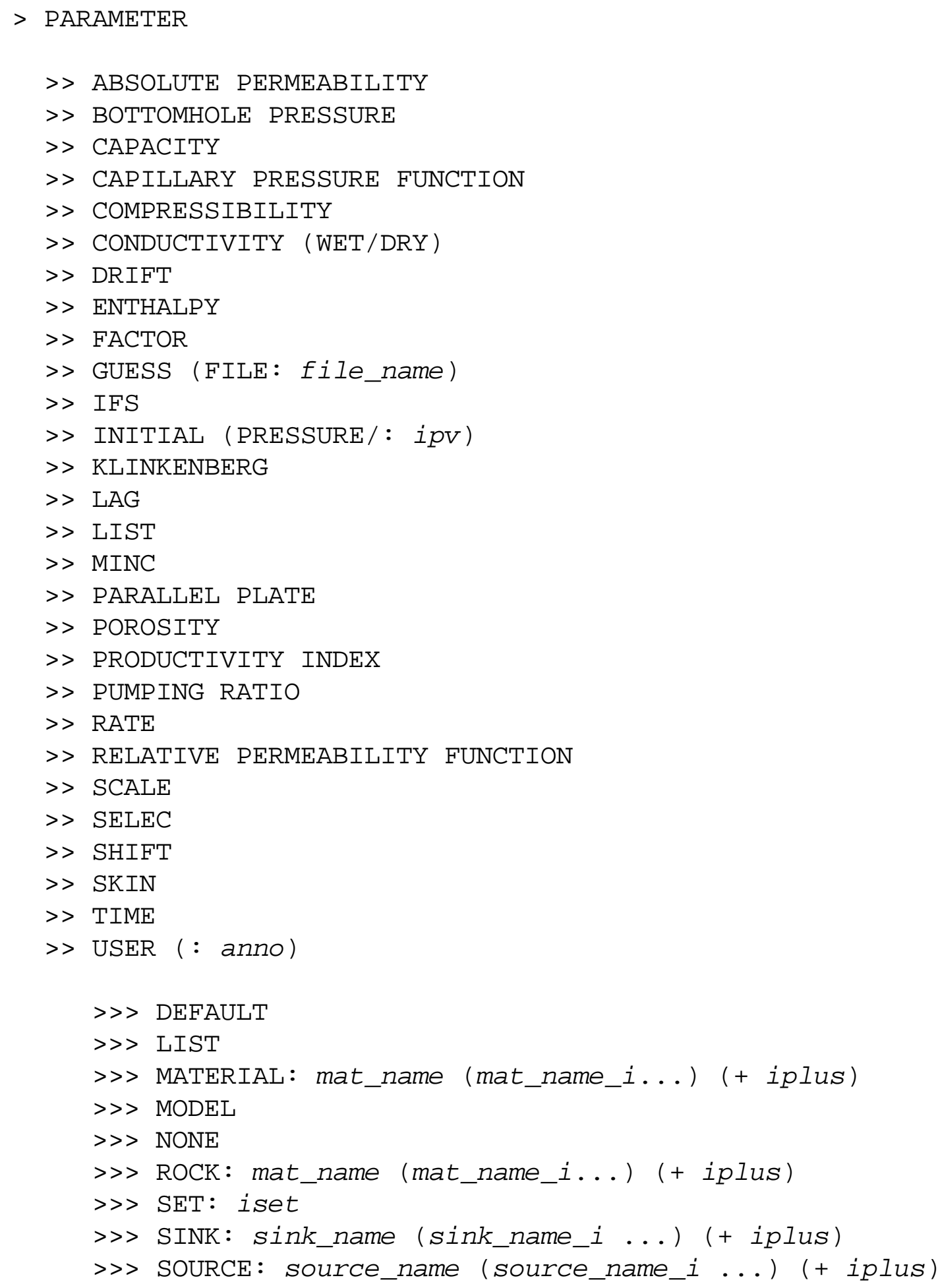




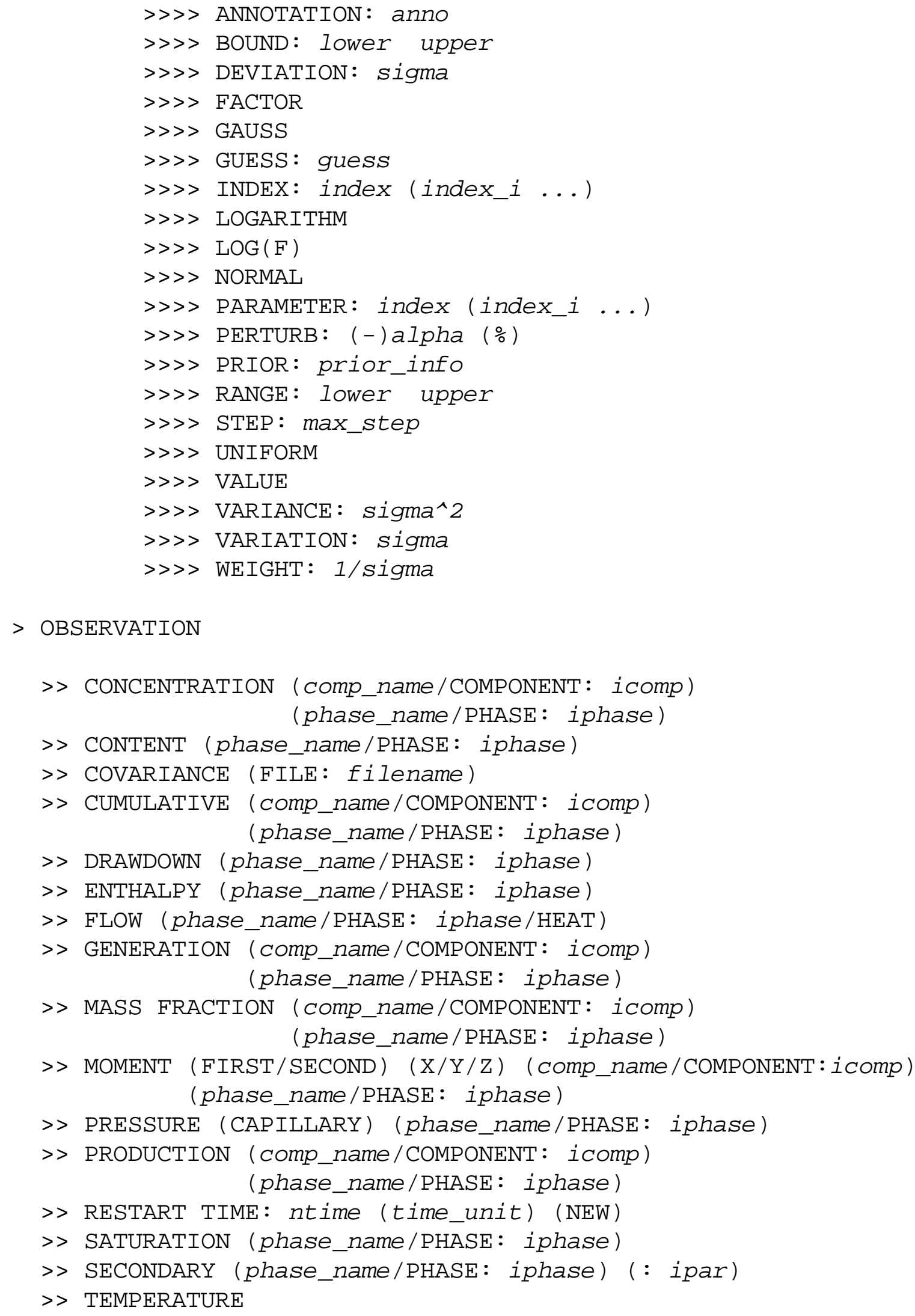




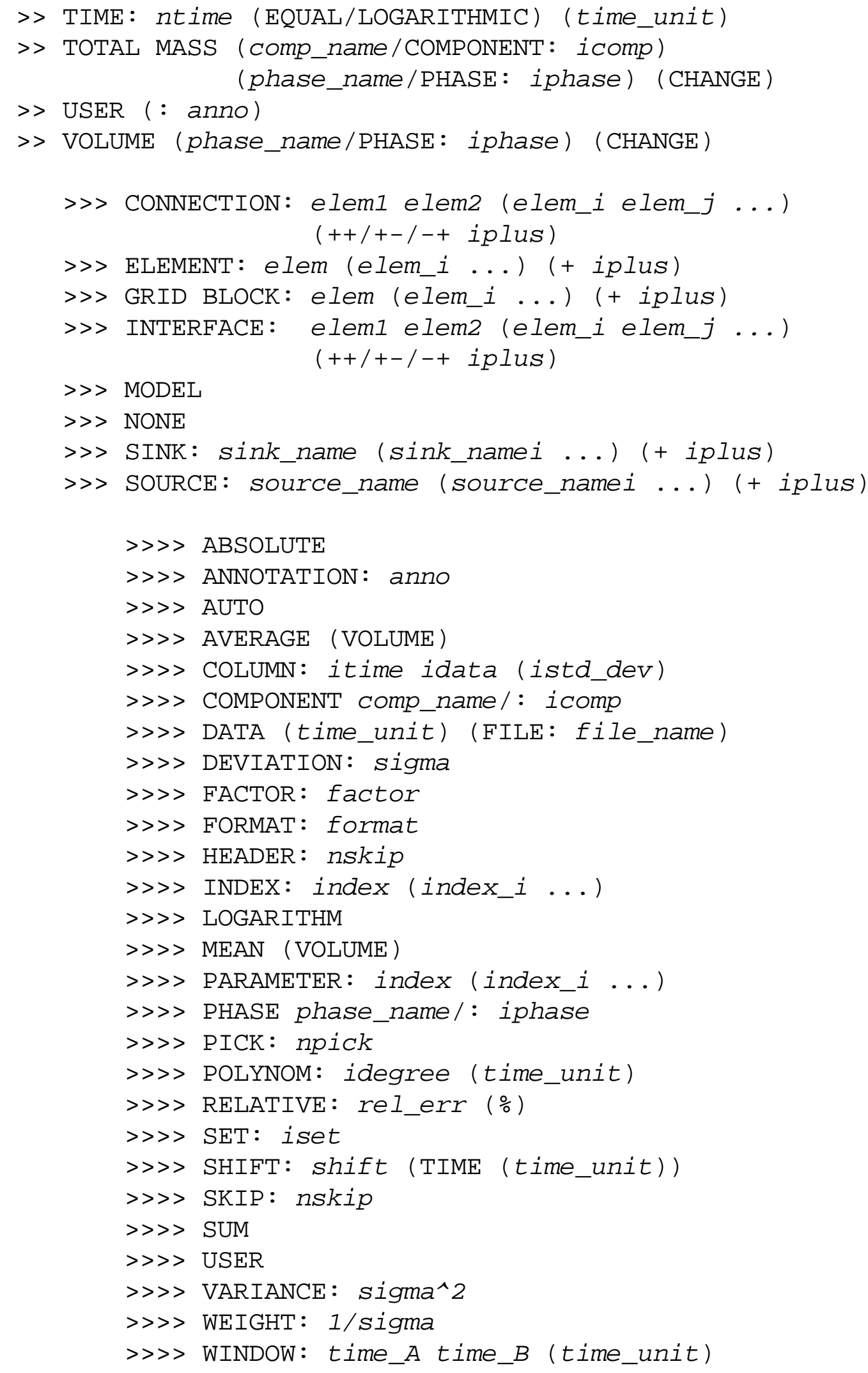




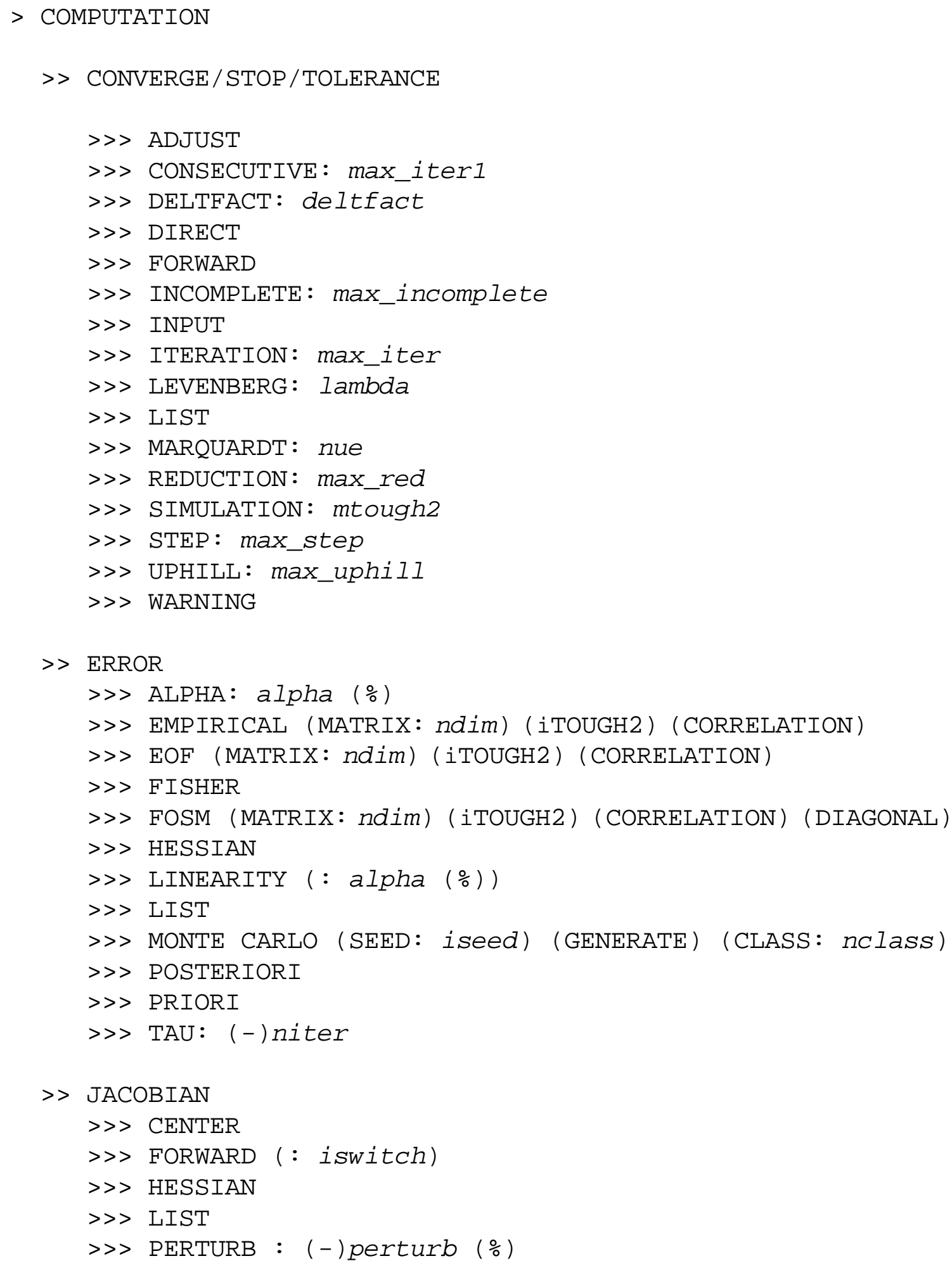




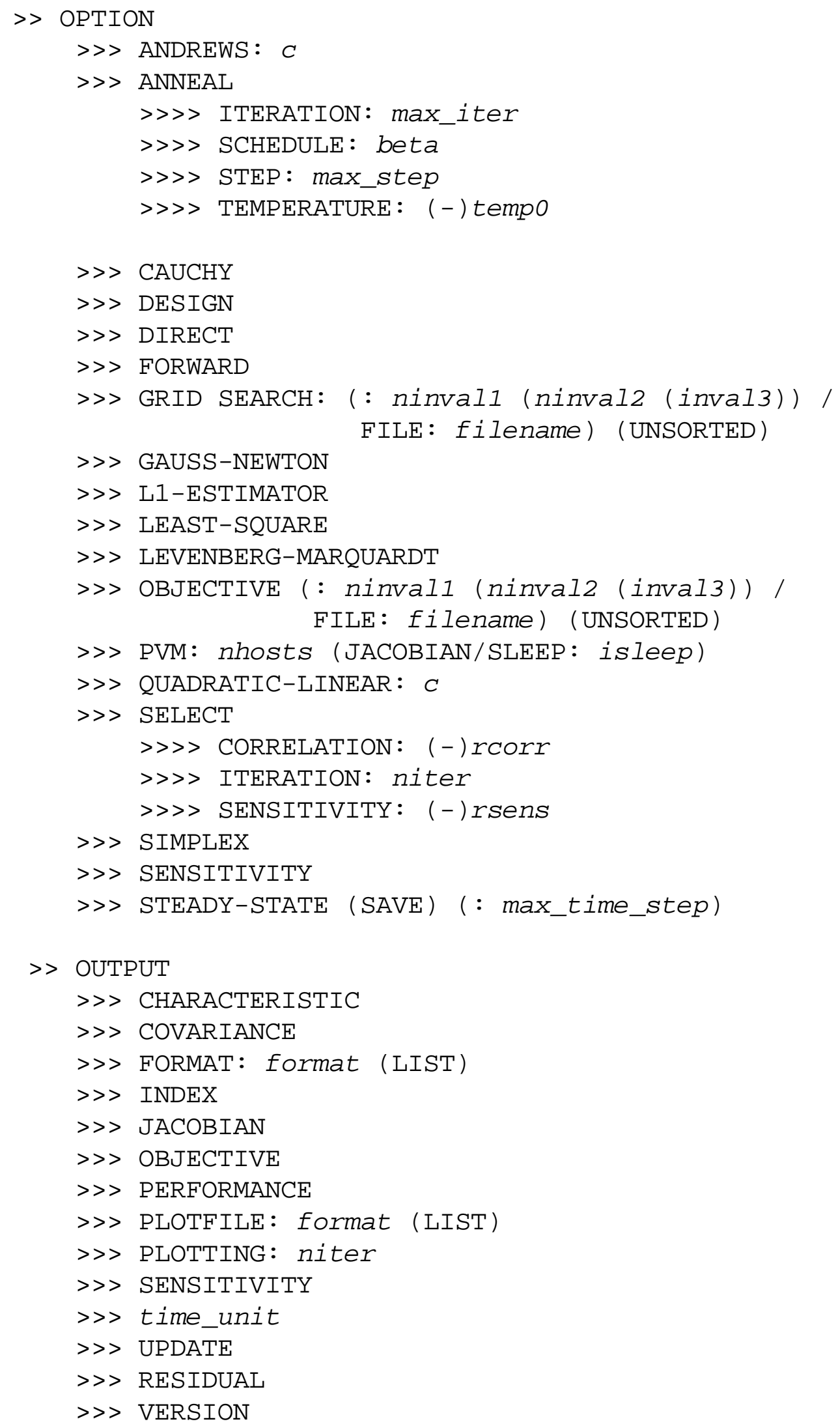

